Lehrstuhl für Kommunikation und Navigation

Technische Universität München

\title{
Partial Carrier-Phase Integer Ambiguity Resolution for High Accuracy GNSS Positioning
}

\author{
Andreas Brack
}

Vollständiger Abdruck der von der Fakultät für Elektrotechnik und Informationstechnik der Technischen Universität München zur Erlangung des akademischen Grades eines

$$
\text { Doktor-Ingenieurs }
$$

genehmigten Dissertation.

Vorsitzender: Prof. Dr.-Ing. Eckehard Steinbach Prüfer der Dissertation: 1. Prof. Dr.sc.nat. Christoph Günther

2. Prof. Dr.ir. Peter J.G. Teunissen (schriftliche Beurteilung) Prof. Dr.phil.nat Urs Hugentobler (mündliche Prüfung)

Die Dissertation wurde am 24.01.2019 bei der Technischen Universität München eingereicht und durch die Fakultät für Elektrotechnik und Informationstechnik am 16.04.2019 angenommen. 



\section{Abstract}

The objective of global navigation satellite system (GNSS) positioning is to provide fast-ideally instantaneous-results at the highest possible accuracy. This can be achieved by utilizing the carrierphases of the signals, which can be tracked at millimeter level precision by the user receiver. Due to the periodicity of the carrier signal, carrier-phase based ranging measurements are ambiguous by unknown integer numbers of complete cycles. Successful carrier-phase integer ambiguity resolution transforms these measurements into very precise ranges, thus enabling centimeter or even millimeter level positioning.

Imposing a constraint on the maximum allowable failure rate when jointly resolving the full set of estimable integer ambiguity parameters can severely limit the availability of ambiguity fixed results, so that long convergence times may be required. The fundamental idea of partial ambiguity resolution is to resolve a subset of the integer ambiguity parameters. This should be beneficial, since it is generally more likely that a subset of ambiguity parameters can be reliably resolved rather than the full set, but this also means that the integer property of the ambiguities and therefore the high precision of the carrier-phase measurements is not fully exploited.

In this dissertation, possible approaches to the problem of partial ambiguity resolution are developed and discussed. A general mathematical framework for estimating subsets of integer parameters in linear observation models is introduced. Optimal and suboptimal estimators are formulated and compared, and implementation aspects are discussed. The impact of partial ambiguity resolution techniques on the positioning capabilities is thoroughly analyzed for various positioning cases both with simulated and real GNSS data. The results show that partial ambiguity resolution can significantly increase the range for instantaneous centimeter level positioning in terms of the distances to nearby reference stations, and otherwise clearly reduce the time required to reach centimeter level accuracy compared to full ambiguity resolution. 



\section{Contents}

$\begin{array}{ll}\text { Acronyms and Notation } & 9\end{array}$

1. Introduction $\quad 13$

1.1 Satellite Navigation Systems . . . . . . . . . . . . . . . . . . . . . 14

1.2 Problem Description, Objectives, and Contribution . . . . . . . . . . . . 16

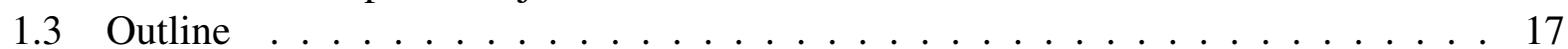

2. GNSS Positioning Models and Parameter Estimation 19

2.1 GNSS Observation Equations . . . . . . . . . . . . . . . . . . . . . . . 19

2.1.1 Undifferenced Measurements . . . . . . . . . . . . . . . . . . . . . . . . . . . 219

2.1.2 Between Receiver Differences . . . . . . . . . . . . . . . . . . . . 21

2.1.3 Between Satellite Differences . . . . . . . . . . . . . . . . . 22

2.1.4 Between Receiver and Satellite Differences . . . . . . . . . . . . . . . 23

2.2 Functional Models . . . . . . . . . . . . . . . . . . . 23

2.2.1 Undifferenced Model . . . . . . . . . . . . . . . . . . . . 24

2.2.2 Single Difference Models . . . . . . . . . . . . . . . . 30

2.2 .3 Double Difference Model . . . . . . . . . . . . . . . . . . 34

2.2.4 Combined Multi-GNSS model . . . . . . . . . . . . . . . . 34

2.3 Stochastic Models . . . . . . . . . . . . . . . . . . . . . 35

2.4 Integer/Mixed Integer and Real Valued Parameter Estimation . . . . . . . . . . . 37

2.5 Atmospheric Delay Parameters . . . . . . . . . . . . . . . . . . . . 39

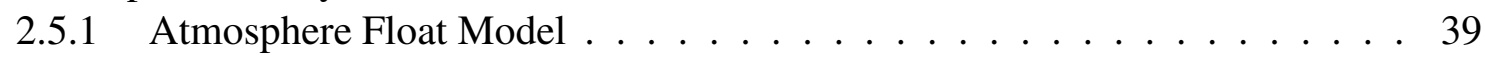

2.5.2 Atmosphere Fixed Model . . . . . . . . . . . . . . . . . . 40

2.5.3 Atmosphere Weighted Model . . . . . . . . . . . . . . . . . 40

2.6 Equivalence of Positioning Models . . . . . . . . . . . . . . . . . . . 41

2.7 Two Step Estimation, PPP-RTK . . . . . . . . . . . . . . . . . . . . . . . . . . . . . . . . . . .

2.8 Model Validation, Measurement Integrity . . . . . . . . . . . . . . . 45

3. Full Ambiguity Resolution-a Review $\quad 47$

3.1 The Class of Integer Estimators . . . . . . . . . . . . . . . . . . . . 47

3.1.1 Quality of the Estimates . . . . . . . . . . . . . . . . 48

3.1 .2 Element-Wise Rounding . . . . . . . . . . . . . . . . . . . 49

3.1 .3 Integer Bootstrapping . . . . . . . . . . . . . . . . . 49

3.1 .4 Integer Least-Squares . . . . . . . . . . . . . . . . . . 50

3.1.5 Decorrelation/Reduction Transformations . . . . . . . . . . . . . 52

3.2 The Class of Integer Aperture Estimators . . . . . . . . . . . . . . . 53

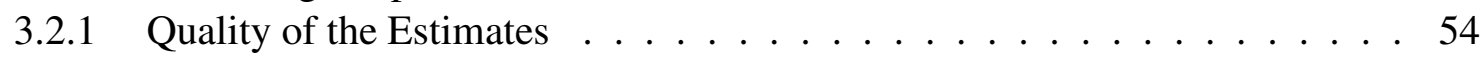

3.2 .2 Optimal Integer Aperture Estimation . . . . . . . . . . . . . . 55

3.2 .3 The Difference Test . . . . . . . . . . . . . . . . 56

3.2 .4 The Ratio Test . . . . . . . . . . . . . . . . . . 57 
3.2.5 The Projector Test . . . . . . . . . . . . . . . 58

4. Partial Integer Estimation $\quad 59$

4.1 The Class of Partial Integer Estimators . . . . . . . . . . . . . . . . . . . 59

4.2 Quality of the Estimates . . . . . . . . . . . . . . . . . . 60

4.3 Examples of Partial Integer Estimators . . . . . . . . . . . . . . . . . . . . . . . . . . . . . 62

4.3.1 Partial Integer Least-Squares . . . . . . . . . . . . . . . . . . . 62

4.3.2 Full Integer Least-Squares + Selection . . . . . . . . . . . . . . . . 63

4.3 .3 Optimal Partial Integer Estimation . . . . . . . . . . . . . . . . 65

4.3.4 Computation of the Optimal Estimator . . . . . . . . . . . . . . . 67

4.3.5 Optimal vs. Suboptimal Partial Integer Estimation . . . . . . . . . . . . 68

4.4 Selecting the Integer Transformation and Subset . . . . . . . . . . . . . . . 70

4.4.1 Integer Transformations for Partial Ambiguity Resolution . . . . . . . . . 70

4.4.2 Subset Selection Criteria . . . . . . . . . . . . . . . . . 71

4.4 .3 Subset Selection Strategies . . . . . . . . . . . . . . . . 72

5. Generalized Integer Aperture Estimation $\quad \mathbf{7 5}$

5.1 The Class of Generalized Integer Aperture Estimators . . . . . . . . . . . . 75

5.2 Quality of the Estimates . . . . . . . . . . . . . . . . . . . . 77

5.3 Single-Subset Generalized Integer Aperture Estimators . . . . . . . . . . . . . . 79

5.3.1 Optimal Single-Subset Generalized Integer Aperture Estimation . . . . . . 79

5.3.2 Difference Test Based Single-Subset Generalized Integer Aperture Estimation . . . . . . . . . . . . . . . . 81

5.3.3 Selecting the Integer Transformation and Subset $\mathcal{I}^{\prime} \ldots \ldots$. . . . . . . . 83

5.4 Multiple-Subset Generalized Integer Aperture Estimators . . . . . . . . . . . . . . 84

5.4.1 Per Element Difference Test . . . . . . . . . . . . . . . . . 85

5.4 .2 Per Element Ratio Test . . . . . . . . . . . . . . . . . . . . . . 87

5.4 .3 Per Element Projector Test . . . . . . . . . . . . . . . . . . . 87

5.4 .4 Computational Aspects . . . . . . . . . . . . . . . . . 88

5.4 .5 Selecting the Integer Transformation . . . . . . . . . . . . . . . . 90

5.4.6 Comparison and Performance Evaluation . . . . . . . . . . . . . . 90

5.5 Fixed Failure Rate Critical Values . . . . . . . . . . . . . . . . . . . . 94

5.5.1 Relation Between Critical Value and Failure Rate . . . . . . . . . . . . . 94

5.5.2 Functional Approximation of Critical Values . . . . . . . . . . . . . . 96

5.5.3 Approximated vs. True Fixed Failure Rate Critical Values . . . . . . . . . 100

6. Reliable GNSS Positioning-Experimental Analysis $\quad 103$

6.1 Positioning Precision, Ambiguity Reliability, and Time . . . . . . . . . . . . . 104

6.2 Numerical Simulations . . . . . . . . . . . . . . . . . . . . 108

6.2 .1 Short Baselines . . . . . . . . . . . . . . . . . . . . . . . . . . . . . . . . . . . . . . . .

6.2 Medium-Length Baselines . . . . . . . . . . . . . . . . . . . . . 109

6.2 .3 Long Baselines . . . . . . . . . . . . . . . . . . . . . . 112

6.2 .4 Multiple Baselines . . . . . . . . . . . . . . . . . . . . . . . . . . . . . . . . . . . . . . . . .

6.3 Real-Data Analysis . . . . . . . . . . . . . . . . . . 115

6.3.1 Variance Component Estimation . . . . . . . . . . . . . . 116

6.3.2 Instantaneous RTK Positioning on CUT0-PERT . . . . . . . . . . . . . . 118 
6.3.3 Long Baseline RTK Positioning on PERT-NNOR . . . . . . . . . . . . 123

7. Integer Equivariant Estimation $\quad 129$

7.1 The Class of Integer Equivariant Estimators ～. . . . . . . . . . . . . . . . . 129

7.2 Examples of Integer Equivariant Estimators . . . . . . . . . . . . . . . . 130

7.2.1 Best Integer Equivariant Estimation . . . . . . . . . . . . . . . . . 131

7.2.2 Parallel Scalar Approximation . . . . . . . . . . . . . . . 132

7.2.3 Sequential Scalar Approximation _ . . . . . . . . . . . . 135

7.3 GNSS Positioning Performance . . . . . . . . . . . . . . . 137

8. Summary and Conclusions 141

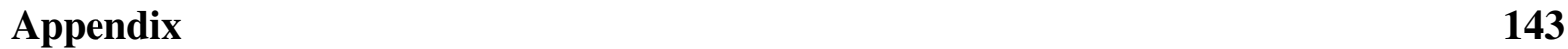

A1. S-System Theory . . . . . . . . . . . . . . . . . . . . . 143

A2. Sufficient Statistics . . . . . . . . . . . . . . . . . . 144

A3. Free $y^{\mathrm{R}}$ Variates . . . . . . . . . . . . . . . . . . . . 146

A4. Constrained Optimization . . . . . . . . . . . . . . . . . 147

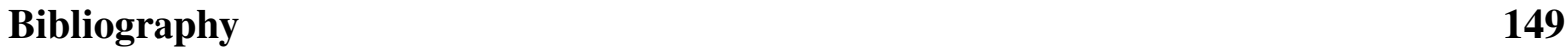





\section{Acronyms and Notation}

\section{Acronyms}

\begin{tabular}{|c|c|}
\hline ADOP & Ambiguity dilution of precision \\
\hline BDS & BeiDou navigation satellite system \\
\hline $\mathrm{BIE}$ & Best integer equivariant \\
\hline BLUE & Best linear unbiased estimator \\
\hline $\mathrm{C} / \mathrm{A}$ & Coarse acquisition \\
\hline CDMA & Code division multiple access \\
\hline CORS & Continuously operating reference station \\
\hline $\mathrm{CoS}$ & Commercial service \\
\hline $\mathrm{CS}$ & Complementary slackness \\
\hline DF & Dual feasibility \\
\hline DIA & Detection, identification, and adaptation \\
\hline DT & Difference test \\
\hline FAR & Full ambiguity resolution \\
\hline FDMA & Frequency division multiple access \\
\hline GEO & Geostationary Earth orbit \\
\hline GNSS & Global navigation satellite system \\
\hline GPS & Global Positioning System \\
\hline GSO & Geo-synchronous orbit \\
\hline HEO & Highly-inclined elliptical orbit \\
\hline IB & Integer bootstrapping \\
\hline IGS & International GNSS service \\
\hline IGSO & Inclined geo-synchronous orbit \\
\hline ILS & Integer least-squares \\
\hline IR & Integer rounding \\
\hline IRNSS & Indian Regional Navigation Satellite System \\
\hline KKT & Karush-Kuhn-Tucker \\
\hline LAMBDA & Least-squares ambiguity decorrelation adjustmen \\
\hline LLR & Log-likelihood ratio \\
\hline MEO & Medium Earth orbit \\
\hline MGEX & Multi GNSS experiment \\
\hline MIMO & Multiple-input multiple-output \\
\hline MOPS & Minimum operational performance standard \\
\hline NavIC & Navigation Indian Constellation \\
\hline OS & Open service \\
\hline PAR & Partial ambiguity resolution \\
\hline pdf & Probability density function \\
\hline $\mathrm{PF}$ & Primal feasibility \\
\hline pmf & Probability mass function \\
\hline
\end{tabular}




$\begin{array}{ll}\text { PPP } & \text { Precise point positioning } \\ \text { PPP-RTK } & \text { Ambiguity resolution enabled precise point positioning } \\ \text { PRS } & \text { Public regulated service } \\ \text { PS } & \text { Precision service } \\ \text { QZSS } & \text { Quasi-Zenith Satellite System } \\ \text { RAIM } & \text { Receiver autonomous integrity monitoring } \\ \text { RTK } & \text { Real-time kinematic } \\ \text { SPP } & \text { Single point positioning } \\ \text { SPS } & \text { Standard positioning service }\end{array}$

\section{Notation}

$x, X$
$\boldsymbol{x}$
$\boldsymbol{X}$
$x_{i}$
$X_{i j}$
[]
$\boldsymbol{e}_{k}$
$\boldsymbol{c}_{i}$
$\mathbf{0}, \mathbf{0}_{k \times n}$
$\boldsymbol{I}, \boldsymbol{I}_{k}$
$\mathbb{R}$
$\mathbb{R}+$
$\mathbb{Z}$
$\{x, y, \ldots\}$
$\emptyset$
$|x|$
$\lfloor x\rceil$
$\operatorname{dim}(\boldsymbol{x})$
$\|\boldsymbol{x}\|$
$\|\boldsymbol{x}\|_{\boldsymbol{Q}}$
$\operatorname{det}(\boldsymbol{X})$
$\operatorname{rank}(\boldsymbol{X})$
$\operatorname{tr}(\boldsymbol{X})$
$\mathcal{R}(\boldsymbol{X})$
$\mathcal{N}(\boldsymbol{X})$
$\operatorname{diag}(x, y, \ldots)$
$\operatorname{blkdiag}(\boldsymbol{X}, \boldsymbol{Y}, \ldots)$
$|\mathcal{X}|$
$\operatorname{int}(\mathbb{X})$
$\geq$
$\succeq$
$\subseteq$

Scalar

Column vector

Matrix

$i$ th element of column vector $\boldsymbol{x}$

$i$ th row, $j$ th column element of matrix $\boldsymbol{X}$

Zero dimensional vector

$k$ dimensional vector of ones

Canonical unit vector with 1 as its $i$ th entry

All zeros vector or matrix of size $k \times n$ (size parameter optional)

Unit matrix of size $k$ (size parameter optional)

Set of real numbers

Set of non-negative real numbers

Set of integers

Discrete set

Empty set

Absolute value of a scalar

Rounding to the nearest integer

Dimension of a vector

Euclidean norm of a vector

Weighted norm $\sqrt{\boldsymbol{x}^{\mathrm{T}} \boldsymbol{Q}^{-1} \boldsymbol{x}}$ of a vector with $\boldsymbol{Q}$ a positive definite matrix

Determinant of a matrix

Rank of a matrix

Trace of a square matrix

Range space of a matrix

Null space of a matrix

Diagonal matrix

Blockdiagonal matrix

Cardinality of a set

Interior of a point set

Element-wise greater

Semidefinite

Included in 


$\propto$
$\otimes$
$\oplus$
$\exp (x)$
$\log (x)$
$\operatorname{sign}(x)$
$\max$
$\min$
$\operatorname{argmax}$
$\operatorname{argmin}$
$P(\cdot)$
$\mathrm{E}[\boldsymbol{x}]$
$\operatorname{Var}[\boldsymbol{x}]$
$\operatorname{MSE}[\boldsymbol{x}]$
$p_{\boldsymbol{x}}(\cdot)$
$\sim \mathcal{G}(\boldsymbol{\mu}, \boldsymbol{Q})$

Proportional to

Kronecker product

Direct sum

Exponential function

Natural logarithm

Signum function

Maximum

Minimum

Maximizing argument

Minimizing argument

Probability of an event

Expectation of a random variable

Variance of a random variable

Mean square error of a random variable

Probability density function of a random variable

Gaussian distributed with mean vector $\boldsymbol{\mu}$ and covariance matrix $\boldsymbol{Q}$ 



\section{Introduction}

Satellite navigation systems are an indispensable source for positioning and timing information that various applications in fields such as navigation, guidance, and surveying rely on. Caused by the diversity of positioning applications, the accuracy and integrity requirements on the solution differ greatly, thus asking for different processing strategies of the received signals. Currently, the vast majority of satellite navigation receivers make use of single point positioning (SPP), in which only the code-based pseudoranges of at least four satellites obtained by the user receiver are employed. The resulting positioning accuracy is at the level of a few meters. Slightly more accurate results are possible when using a second nearby receiver in a differential mode, which greatly reduces the influence of atmospheric and residual satellite orbit and clock errors.

In order to obtain a positioning accuracy at the centimeter or even sub-centimeter level, it is essential to include carrier-phase observations. They are roughly two orders of magnitude less noisy compared to code observations, but are ambiguous due to the unknown integer multiples of a complete cycle caused by the periodicity of the carrier-signal. When estimating the user position, we therefore have to introduce additional parameters for these so called carrier-phase integer ambiguities in the observation model. Two fundamental positioning techniques can be distinguished in this context, namely precise point positioning (PPP) and real-time kinematic (RTK) positioning.

The concept of PPP is to provide precise satellite orbit and clock data to the user receiver. However, without satellite phase bias corrections, the carrier-phase integer ambiguities cannot be separated from instrumental satellite biases in the observation model when using data from a single receiver, so that we have to use combined parameters containing instrumental delays and integer ambiguities. Because of the instrumental delays, these combinations are real valued and resolving any ambiguities as integers is not possible. Static PPP is capable of reaching centimeter level positioning accuracy but requires a convergence time of several hours (Zumberge et al. 1997; Kouba and Héroux 2001; Bisnath and Gao 2008).

In RTK positioning, the measurements of at least two receivers are processed jointly, so that certain linear combinations of the carrier-phase ambiguities can be isolated from the satellite instrumental biases in the observation model and are now estimable as integers. These combinations are usually chosen as the double difference ambiguities with a common pivot satellite and receiver. By correctly resolving these combinations to their true integer values we can fully exploit the high precision of the carrier-phase observations and immediately get very accurate positioning results without long convergence times. The RTK positioning capabilities are, however, limited by the need for correct estimates of the carrier-phase ambiguities, since incorrect ambiguity estimates are likely to result in large errors of the estimated user position. Ideally, a user-defined maximum tolerable failure rate is not exceeded. This can be accomplished by properly designing the ambiguity resolution scheme.

The concept of PPP can be extended by providing a single receiver user, in addition to satellite orbit and clock data, also with information about the satellite phase biases. If properly provided and applied by the user, these external corrections enable the user to estimate integer valued combinations of the carrier-phase ambiguities like in RTK positioning and thus to perform integer ambiguity resolution. As a result, the long convergence times of conventional PPP can be avoided. 
This combined technique can therefore be described as integer ambiguity resolution enabled PPP or simply PPP-RTK (Wübbena et al. 2005; Laurichesse and Mercier 2007; Mervart et al. 2008).

Because of the high hardware costs of geodetic receivers that are capable of providing carrierphase data, high accuracy satellite positioning is mainly used by professional and scientific users. With the recent availability of low-cost carrier-phase enabled receivers, high precision satellite navigation can be expected to enter the consumer market in the near future and a much wider range of applications can be envisioned. It is demonstrated in Pesyna et al. (2014), Henkel (2015), and Odolinski and Teunissen (2016) that centimeter level positioning is possible with low cost receivers and patch or even smartphone-quality antennas.

The ambiguity resolution capabilities depend on factors such as the number of visible satellites, the relative satellite-receiver geometry, the number of employed frequencies, the noise characteristics of the measurements, and the potential presence of multipath or differential atmospheric delays. Better ambiguity resolution performance can be achieved by strengthening the underlying observation model, e.g., by means of combining multiple satellite navigation systems or improved modeling of atmospheric or instrumental biases. A constraint in form of a maximum tolerable ambiguity failure rate may, however, still hinder fast ambiguity resolution and thereby fast centimeter level positioning. In such cases, it may be beneficial to consider partial ambiguity resolution techniques, which resolve only a subset of the integer ambiguities. Since it is generally more likely that a subset of all estimable integer ambiguities can be reliably resolved rather than the full set, partial ambiguity resolution techniques should lead to improved positioning capabilities like faster solutions or a higher availability of instantaneous centimeter level coordinate estimates. However, we have to keep in mind that the resulting positioning accuracy after successful partial ambiguity resolution is generally lower than the one after successful full ambiguity resolution, since the integer property of the ambiguities is not fully exploited. The following problems have to be faced in the context of partial ambiguity resolution. Given an observation model and a set of measurements, how do we determine the subset of ambiguity parameters to be resolved and what are the corresponding integer estimates? The overall failure rate must thereby not exceed our predefined maximum value. One can think of a vast amount of ways to approach this problem. The goal is to find solutions resulting in a positioning performance that is as good as possible, while keeping the computational complexity compatible with real time applications. This dissertation attempts to extensively answer these questions.

\subsection{Satellite Navigation Systems}

The best known global navigation satellite system (GNSS) is the American Global Positioning System (GPS), which has been fully operational since 1995. Its nominal constellation consists of 24 medium Earth orbit (MEO) satellites, and there are currently (December 2018) 31 satellites in orbit that are marked healthy. The GPS satellites transmit their ranging signals in the frequency bands L1, L2, and L5 using the code division multiple access (CDMA) scheme, so that signals from different satellites share the same carrier-frequency and are separated via unique spreading codes. The carrier-frequencies are chosen as an integer multiple of $10.23 \mathrm{MHz}$, so that they can be derived from the same frequency source. The common wavelength of the signals from all satellites is utilized when formulating integer valued double difference ambiguity parameters. Originally, only the course acquisition (C/A) signal on L1 was available to civil users. Measurements on L2 can be obtained by a civil receiver via codeless or semi-codeless techniques, i.e., without knowing the Y code of the encrypted P(Y) signal (IS-GPS-200 2016). With the block IIR-M satellites 
Table 1.1: Plan of CDMA signals for satellite navigation systems. The multiplier specifies the multiple of the base frequency $10.23 \mathrm{MHz}$.

\begin{tabular}{llllll}
\hline System & Band & Frequency & Multiplier & Wavelength & Signals/Services \\
\hline GPS & L1 & $1,575.420 \mathrm{MHz}$ & 154 & $19.03 \mathrm{~cm}$ & C/A, L1C, P(Y), M \\
& L2 & $1,227.600 \mathrm{MHz}$ & 120 & $24.42 \mathrm{~cm}$ & L2C, P(Y), M \\
\multirow{3}{*}{ GLONASS } & L5 & $1,176.450 \mathrm{MHz}$ & 115 & $25.48 \mathrm{~cm}$ & L5 \\
& L2 & $1,600.995 \mathrm{MHz}$ & 156.5 & $18.73 \mathrm{~cm}$ & L1OC, L1SC \\
& L3 & $1,248.060 \mathrm{MHz}$ & 122 & $24.02 \mathrm{~cm}$ & L2OC, L2SC \\
Galileo & E1 & $1,575.42025 \mathrm{MHz}$ & 117.5 & $24.94 \mathrm{~cm}$ & L3OC \\
& E6 & $1,278.750 \mathrm{MHz}$ & 125 & $23.44 \mathrm{~cm}$ & PRS, CoS \\
& E5 & $1,191.795 \mathrm{MHz}$ & 116.5 & $25.15 \mathrm{~cm}$ & \\
& E5a & $1,176.450 \mathrm{MHz}$ & 115 & $25.48 \mathrm{~cm}$ & OS \\
& E5b & $1,207.140 \mathrm{MHz}$ & 118 & $24.83 \mathrm{~cm}$ & OS \\
BDS & B1 & $1,561.098 \mathrm{MHz}$ & 152.6 & $19.20 \mathrm{~cm}$ & B1I, B1Q \\
& B2 & $1,207.140 \mathrm{MHz}$ & 118 & $24.83 \mathrm{~cm}$ & B2I, B2Q \\
& B3 & $1,268.520 \mathrm{MHz}$ & 124 & $23.63 \mathrm{~cm}$ & B3I, B3Q \\
QZSS & L1 & $1,575.420 \mathrm{MHz}$ & 154 & $19.03 \mathrm{~cm}$ & C/A, L1C, SAIF \\
& L2 & $1,227.600 \mathrm{MHz}$ & 120 & $24.42 \mathrm{~cm}$ & L2C \\
& L5 & $1,176.450 \mathrm{MHz}$ & 115 & $25.48 \mathrm{~cm}$ & L5 \\
& E6 & $1,278.750 \mathrm{MHz}$ & 125 & $23.44 \mathrm{~cm}$ & LEX \\
NavIC & L5 & $1,176.450 \mathrm{MHz}$ & 115 & $25.48 \mathrm{~cm}$ & SPS, PS \\
\hline
\end{tabular}

(launched 2005-2009), the second civil signal L2C on L2 as well as the military M signals became available, and with the block IIF satellites (launched 2010-2016), a third civil signal on L5 was introduced (IS-GPS-705 2014). The block III satellites will transmit the additional civil L1C signal on L1 (IS-GPS-800 2014). A summary of the signals is given in Table 1.1.

A second satellite navigation system with global coverage is the Russian GLONASS, whose constellation was firstly completed in 1995. Different from all other current satellite navigation systems, GLONASS makes use of the frequency division multiple access (FDMA) scheme, meaning that different satellites transmit at slightly different carrier-frequencies (GLONASS-ICD 2008). The inherent inter frequency biases hamper integer ambiguity resolution. However, with the modernization of GLONASS, additional open (OC) and restricted (SC) CDMA signals on L1, L2, and L3 are introduced, see Table 1.1, so that standard methods for integer ambiguity resolution can be applied. Additional L1 and L5 CDMA signals are planned for the future to improve the interoperability with GPS (Urlichich et al. 2011; Oleynik 2012). As of December 2018, the GLONASS constellation consists of 24 operational MEO satellites, of which six are capable of transmitting the L3 CDMA signal.

Another GNSS that is still under construction is the European Galileo system with currently 18 operational satellites and a nominal constellation of 24 MEO satellites plus six spare satellites (OS-SIS-ICD 2016). The Galileo satellites transmit signals in the four frequency bands E1, E6, E5a, and E5b. The Galileo system provides open service (OS) signals on E1, E5a, and E5b that are available for all users and the restricted public regulated service (PRS) and commercial service 
(CoS) signals on E1 and E6, see Table 1.1. The signals on E5a and E5b can be received and used separately or as a single wideband signal.

The Chinese BeiDou Navigation Satellite System (BDS) was primarily designed to provide a regional positioning service only in the Asia-Pacific region. As of December 2018, it consists of six geostationary Earth orbit (GEO) satellites and seven inclined geo-synchronous orbit (IGSO) satellites that are only visible in that region, and of five MEO satellites. In a second phase, BDS will provide a global positioning service with a final constellation that is planned to consist of five GEO, three IGSO, and 27 MEO satellites (BDS-SIS-ICD 2016). The BDS satellites transmit their ranging signals as the inphase and quadrature components on three carriers in the B1, B2, and B3 frequency bands. The inphase components B1I and B2I in B1 and B2 are intended for civil users and are specified in BDS-SIS-ICD (2016).

A purely regional satellite navigation system is the Japanese Quasi-Zenith Satellite System (QZSS) with a nominal constellation of three or more highly-inclined elliptical orbit (HEO) satellites, of which currently four are orbit. The goal of QZSS is to provide a constellation that is optimized to enhance the availability of standalone GPS in Japan. The orbits are therefore designed such that the satellites are visible over Japan at high elevation angles for preferably long time intervals. In order to maximize the interoperability with GPS, the QZSS signals C/A, L1C, L2C, L5 are defined very similar as for GPS, see Table 1.1. The SAIF and LEX signals are used to transmit integrity data (IS-QZSS 2016).

The Indian Regional Navigation Satellite System (IRNSS) or Navigation Indian Constellation (NavIC) is designed to provide a navigation service in India and in regions within 1,500 $\mathrm{km}$ from its borders. The full constellation of three GEO and four geo-synchronous orbit (GSO) satellites was completed in 2016. NavIC provides an open standard positioning service (SPS) and a restricted precision service (PS) signal in the L5 band, see Table 1.1, and in the S band at a carrier-frequency of 2,492.028 MHz (ISRO-IRNSS-ICD-SPS 2014).

\subsection{Problem Description, Objectives, and Contribution}

From a mathematical point of view, carrier-phase based positioning with integer ambiguity resolution is an adjustment or estimation problem in a linear model with mixed integer and real valued parameters. The integer ambiguities are thereby nuisance parameters, whose estimation has the only purpose to improve the quality of the estimated non-integer parameters such as the user position. The problem of how and when to resolve the full set of integer ambiguities has been extensively studied in the literature. An overview of theory and methods is presented in, e.g., Teunissen (2003d) and Verhagen (2005).

The goal of this dissertation is to systematically extend the work that has been done on the topic of full ambiguity resolution to the problem of partial ambiguity resolution. In particular, the two above mentioned questions of how do we select the subset of ambiguities to be resolved and what is the integer solution for this subset are addressed. To this end, the following specific topics are covered.

- An estimator for resolving a subset of integer parameters in a linear model has to fulfill certain properties in order to be admissible to this estimation problem. Depending on which desirable properties we ask from the estimator, we can think of different classes of estimators that can be defined by means of the restrictions that have to be imposed when formulating a specific estimator. Two classes of estimators for resolving a subset of integer parameters in a linear 
model are defined in this dissertation. They differ in how the subset can be selected. For the class of partial integer estimators, the subset of integer parameters to be resolved is a priori fixed so that for a given situation always the same subset of integer parameters is resolved. With the class of generalized integer aperture estimators a more flexible approach is introduced, in which the choice of the subset is not deterministic but also results from the estimator so that it may vary for a given situation depending on the measurement data.

- The optimal partial integer estimator for a given subset resulting in the largest possible probability of correct integer estimates is derived. For the class of generalized integer aperture estimators, optimality is very hard to define due to the joint determination of subset and integer solution. That is, different estimators of that class may lead to different subsets so that the results are difficult to compare. The optimal generalized integer aperture estimator is only derived for the special case that either an a priori chosen subset of integer parameters is resolved or not.

- Further non-optimal estimators for both classes are formulated and compared using measures like the success rate for a given subset or the average ratio of resolved integer parameters under a failure rate constraint.

- Algorithms and methods for a computationally efficient implementation of the estimators found are discussed. This includes subset selection strategies, integer decorrelation methods, treesearch formulations with proper choices of the search domains, and functional approximations of fixed failure rate critical values.

- An exhaustive investigation of the capabilities of partial ambiguity resolution techniques in comparison to full ambiguity resolution for high accuracy GNSS positioning is performed for various simulated GNSS positioning cases, particularly with regard to upcoming multi-GNSS solutions. The benefit of partial ambiguity resolution is verified with real GPS and BDS data that was recorded in the area of Perth, Australia, where both constellations were already fully operational.

\subsection{Outline}

This dissertation is structured as follows. In Chapter 2, the fundamental GNSS code and carrierphase observation equations are presented. The system models used for single baseline RTK positioning with undifferenced and between receiver or/and satellite differenced measurements are formulated. A basic framework for solving these positioning problems is introduced. All methods for full and partial ambiguity resolution presented in later chapters can be embedded in this common estimation framework. Atmospheric delay parameters, PPP-RTK, and model validation are briefly discussed.

In Chapter 3, a review of the existing theory of full ambiguity resolution is given. The classes of integer estimators and integer aperture estimators are defined and the respective optimal estimators and further prominent examples are presented. Some of the basic ideas and concepts in this chapter are again used in modified form in the theory and methods for partial ambiguity resolution as developed in Chapters 4 and 5.

The extension of the class of integer estimators so as to be capable of resolving an arbitrary but deterministic subset of the integer parameters is defined in Chapter 4. Different examples of this class of partial integer estimators, including the optimal estimator, are presented and criteria and strategies for selecting the subset of integer ambiguities to be resolved in GNSS positioning applications are discussed. 
Similarly, the class of generalized integer aperture estimators as defined in Chapter 5 is an extension of the class of integer aperture estimators that is capable of resolving subsets of the integer parameters. Various examples of generalized integer aperture estimators are discussed and a user friendly way to control the associated failure rates is developed.

A thorough analysis of the GNSS positioning capabilities with full and partial ambiguity resolution techniques and a maximum failure rate constraint is presented in Chapter 6 using simulated and real GNSS data. The main goal of this chapter is to determine if, when, and to what extent partial ambiguity resolution techniques are beneficial towards the ultimate goal of an instantaneous global positioning service with centimeter level accuracy.

In Chapter 7, an alternate approach for solving the positioning model by means of integer equivariant estimation is presented. The fundamental idea is to utilize the integer property of the ambiguities without fixing them to integers. A sequential and a parallel scalar approximation of the existing best integer equivariant estimator are proposed. 


\section{GNSS Positioning Models and Parameter Estimation}

In this chapter the basic framework for determining a user position with the help of satellite navigation systems is presented. We start with a description of the two fundamental types of observables that are used for precise GNSS positioning in Section 2.1: The code-based pseudoranges and the carrier-phases, which can be expressed both in undifferenced form and as between receiver or/and satellite differences. On the basis of these observables various types of models for GNSS parameter estimation can be set up, not all of which include the estimation of coordinates. We restrict ourselves to positioning applications, where the coordinates of a user receiver are determined from the measurements of at least two GNSS receivers. The corresponding mathematical models are described in Sections 2.2 and 2.3. The system model is shown to contain both integer and real valued parameters, which requires non-standard adjustment methods. A general strategy to solve this problem is derived in Section 2.4. This strategy is the basis for the integer estimation schemes presented in the following chapters. Different strategies for dealing with atmospheric delays are shown in Section 2.5. Equivalence properties of different observation models are discussed in Section 2.6. The basic concepts of single receiver ambiguity resolution enabled positioning and model validation are presented in Sections 2.7 and 2.8.

\subsection{GNSS Observation Equations}

We formulate the code and carrier-phase observation equations that are required to use measurement data from GNSS receivers. The receiver internal processing that produces these measurements is not discussed. The measurements are linked to geometrical and physical parameters such as receiver and satellite coordinates, instrumental-, and atmospheric delays. It is assumed that all observations are from a single GNSS. The modifications required to cope with observations from multiple GNSS simultaneously are discussed in Section 2.2.4. For more detailed derivations of the observation equations the reader is referred to textbooks such as Teunissen and Kleusberg (2012) and Leick et al. (2015).

\subsubsection{Undifferenced Measurements}

The code measurement $p_{r, f}^{s}(t)$ at time $t$ at the carrier-frequency $f$ is generated by the receiver by measuring the difference between the time of signal transmission at satellite $s$ and the time of signal reception at receiver $r$. The carrier-phase measurement $\phi_{r, f}^{s}(t)$ is obtained by taking the difference between the phase of the receiver generated carrier signal at frequency $f$ at the time of signal reception and the phase of the satellite generated carrier signal at the time of transmission. A functional description of $p_{r, f}^{s}(t)$ and $\phi_{r, f}^{s}(t)$ can be given by

$$
\begin{gathered}
p_{r, f}^{s}(t)=\rho_{r}^{s}\left(t, t-\tau_{r}^{s}\right)+c\left(d t_{r}(t)-d t^{s}\left(t-\tau_{r}^{s}\right)+d_{r, f}(t)-d_{, f}^{s}\left(t-\tau_{r}^{s}\right)\right) \\
\quad+I_{r, f}^{s}(t)+T_{r}^{s}(t)+\varepsilon_{r, f}^{s}(t) \\
\phi_{r, f}^{s}(t)=\rho_{r}^{s}\left(t, t-\tau_{r}^{s}\right)+c\left(d t_{r}(t)-d t^{s}\left(t-\tau_{r}^{s}\right)+\delta_{r, f}(t)-\delta_{, f}^{s}\left(t-\tau_{r}^{s}\right)\right) \\
\quad-I_{r, f}^{s}(t)+T_{r}^{s}(t)+\lambda_{f} M_{r, f}^{s}+\epsilon_{r, f}^{s}(t)
\end{gathered}
$$


where:

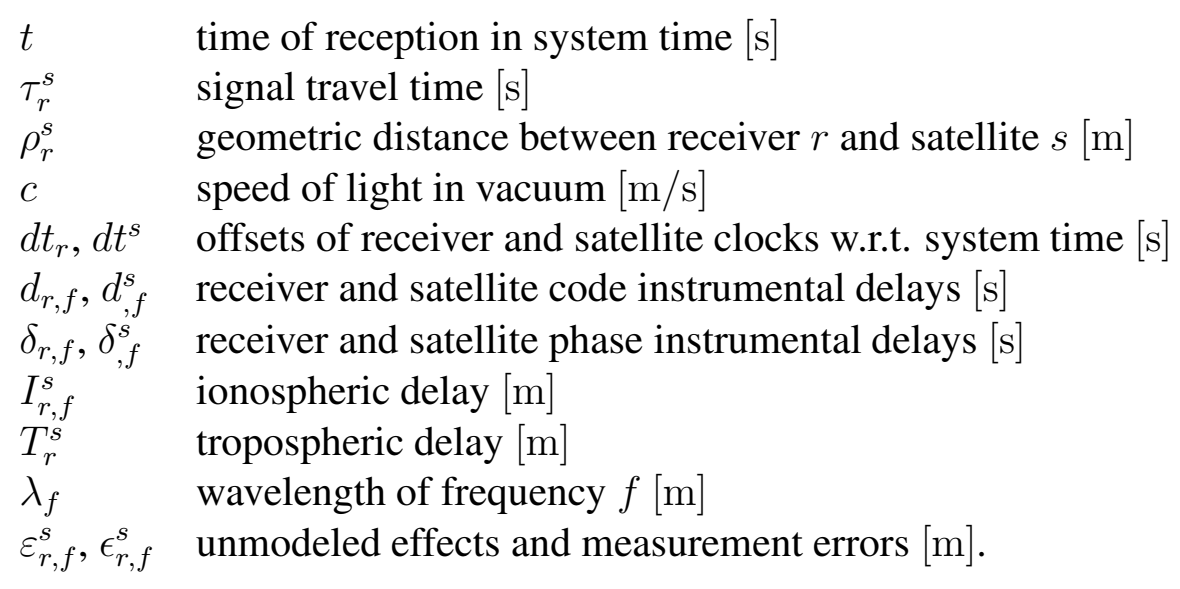

A major difference of the carrier-phase measurement compared to the respective code measurement is the presence of the term

$$
M_{r, f}^{s}=\varphi_{r, f}\left(t_{0}\right)-\varphi_{, f}^{s}\left(t_{0}\right)+N_{r, f}^{s}
$$

where $\varphi_{r, f}\left(t_{0}\right)$ and $\varphi_{, f}^{s}\left(t_{0}\right)$ are the initial phases at the receiver and the satellite in cycles and $N_{r, f}^{s} \in \mathbb{Z}$ is an unknown number of complete cycles, referred to as carrier-phase integer ambiguity. This term is caused by the fact that only the fractional part of the carrier-phase can be measured. The carrier-phase ambiguity is time constant as long as the satellite is tracked without interruption and given that no cycle slip occurs. The initial phases can also be considered as part of the phase instrumental delays $\delta_{r, f}$ and $\delta_{, f}^{s}$.

The clock terms are identical for both code and carrier-phase on all frequencies and are caused by the imperfect synchronization of the local receiver- and satellite clocks with system time. The instrumental delays, on the other hand, are frequency dependent and different for code and carrierphase measurements. The ionosphere parameter $I_{r, f}^{s}$ describes the influence of the plasma contained in the atmosphere in the altitude range from 80 to $1,000 \mathrm{~km}$. The ionosphere is a dispersive medium, which means that the ionospheric effect depends on the frequency $f$ of the signal. It causes a delay in the code measurements and an advance in the carrier-phase measurements, thus the opposite signs in (2.1) and (2.2). The ionospheric effect can be decomposed into a first order term, which can reach values of tens of meters under bad conditions, and higher order terms, which in the double differenced form that is used for differential positioning, see Section 2.2, are at millimeter level for between receiver distances of less than $400 \mathrm{~km}$ and thus usually negligible (Odijk 2002). We describe the ionospheric effect as

$$
I_{r, f}^{s}(t)=\mu_{f} \cdot I_{r}^{s}(t) \quad \text { with } \quad \mu_{f}=\frac{f_{1}^{2}}{f_{f}^{2}}=\frac{\lambda_{f}^{2}}{\lambda_{1}^{2}},
$$

where $I_{r}^{s}(t)$ denotes the ionospheric slant delay on the first frequency. The tropospheric delay $T_{r}^{s}$ describes the influence of the neutral part of the atmosphere between the surface of the Earth and an altitude of roughly $16 \mathrm{~km}$, which is non-dispersive at GNSS frequencies. The tropospheric delay reaches values of up to $2.5 \mathrm{~m}$ in zenith direction. This delay can be decomposed into a hydrostatic and a wet component. The hydrostatic component accounts for roughly $90 \%$ of the delay and can be modeled and a priori corrected very accurately, e.g., with Saastamoinen's model 
(Saastamoinen 1972), which requires knowledge about the surface pressure, or with the blind MOPS model (MOPS 1999). The zenith wet and hydrostatic delays at the receiver location can be projected into slant direction via mapping functions like the Niell mapping function (Niell 1996), the global mapping function (Boehm et al. 2006a), or the Vienna mapping function (Boehm et al. 2006b). The residual tropospheric delays at receiver $r$ can be modeled as the product of a common zenith delay $T_{\mathrm{z}, r}(t)$ and the mapping function $\psi_{r}^{s}(t)$ as

$$
T_{r}^{s}(t)=\psi_{r}^{s}(t) \cdot T_{\mathrm{z}, r}(t)
$$

The geometric range $\rho_{r}^{s}\left(t, t-\tau_{r}^{s}\right)$ can further be expressed as

$$
\rho_{r}^{s}\left(t, t-\tau_{r}^{s}\right)=\left\|\boldsymbol{x}_{r}(t)-\boldsymbol{x}^{s}\left(t-\tau_{r}^{s}\right)\right\|,
$$

where $\boldsymbol{x}_{r}(t) \in \mathbb{R}^{3}$ are the coordinates of receiver $r$ at time $t$ and $\boldsymbol{x}^{s}\left(t-\tau_{r}^{s}\right) \in \mathbb{R}^{3}$ the coordinates of satellite $s$ at time $t-\tau_{r}^{s}$.

The following simplifications have been made when formulating the observation equations:

- The signal travel time $\tau_{r}^{s}$ has been assumed equal for code and carrier-phase measurements on all frequencies. Due to instrumental and atmospheric delays and possible frequency dependent differences in the phase center of the receiver and satellite antennas this is not strictly true. The influence of these effects has been shown in Tiberius (1998) to be negligible when evaluating the time of signal transmission.

- As a consequence, the geometric ranges $\rho_{r}^{s}(t)$ and thus the receiver and satellite coordinates $\boldsymbol{x}_{r}(t)$ and $\boldsymbol{x}^{s}\left(t-\tau_{r}^{s}\right)$ are assumed equal for code and carrier-phase measurements on all frequencies.

- Further non-random effects that are not estimated when solving the positioning problem are not explicitly included in the observation equations, but may have to be considered. For instance, the measurements may be a priori corrected for known antenna phase center offsets and variations. Using identical receiver antennas with the same orientation, these effects are often negligible in the discussed differential positioning setup. A model for the phase wind-up resulting from a rotation of the transmit or receiver antenna based on the geometry is provided in Wu et al. (1993). This effect can usually be safely neglected for differential positioning with between receiver distances of at most several hundred kilometers (Bisnath et al. 2007), but may become relevant for wobbling receivers (Psiaki and Mohiuddin 2007). Similarly, solid Earth tides and other site displacement effects may be a priori corrected or be negligible in the differential setup. Multipath delays can to a certain extent be suppressed with choke ring antennas or advanced receiver signal processing. Residual multipath errors are neglected.

\subsubsection{Between Receiver Differences}

If a satellite is tracked by two receivers simultaneously, the measurement differences between the two receivers can be computed. The differenced code observation equation follows from (2.1) as

$$
\begin{aligned}
p_{r, f}^{s}(t)-p_{q, f}^{s}(t)=\| & \boldsymbol{x}_{r}(t)-\boldsymbol{x}^{s}\left(t-\tau_{r}^{s}\right)\|-\| \boldsymbol{x}_{q}(t)-\boldsymbol{x}^{s}\left(t-\tau_{q}^{s}\right) \| \\
& +c\left(d t_{r}(t)-d t^{s}\left(t-\tau_{r}^{s}\right)+d_{r, f}(t)-d_{, f}^{s}\left(t-\tau_{r}^{s}\right)\right) \\
& -c\left(d t_{q}(t)-d t^{s}\left(t-\tau_{q}^{s}\right)+d_{q, f}(t)-d_{, f}^{s}\left(t-\tau_{q}^{s}\right)\right) \\
& +I_{r, f}^{s}(t)-I_{q, f}^{s}(t)+T_{r}^{s}(t)-T_{q}^{s}(t)+\varepsilon_{r, f}^{s}(t)-\varepsilon_{q, f}^{s}(t) .
\end{aligned}
$$


The difference between the travel times $\tau_{r}^{s}$ and $\tau_{q}^{s}$ is sufficiently small so that the satellite clock offsets $d t^{s}\left(t-\tau_{*}^{s}\right)$ and instrumental delays $d_{, f}^{s}\left(t-\tau_{*}^{s}\right)$ can be considered constant. The satellite coordinates $\boldsymbol{x}^{s}\left(t-\tau_{r}^{s}\right)$ and $\boldsymbol{x}^{s}\left(t-\tau_{q}^{s}\right)$ can, however, be significantly different. With the abbreviation $(\cdot)_{r}-(\cdot)_{q}=(\cdot)_{r q}$, the between receiver differenced code observation can then be rewritten as

$$
\begin{aligned}
p_{r q, f}^{s}(t)=\| & \boldsymbol{x}_{r}(t)-\boldsymbol{x}^{s}\left(t-\tau_{r}^{s}\right)\|-\| \boldsymbol{x}_{q}(t)-\boldsymbol{x}^{s}\left(t-\tau_{q}^{s}\right) \| \\
& +c\left(d t_{r q}(t)+d_{r q, f}(t)\right)+I_{r q, f}^{s}(t)+T_{r q}^{s}(t)+\varepsilon_{r q, f}^{s}(t) .
\end{aligned}
$$

Similarly, the between receiver differenced carrier-phase observation equation follows from (2.2) as

$$
\begin{aligned}
\phi_{r q, f}^{s}(t)=\| & \boldsymbol{x}_{r}(t)-\boldsymbol{x}^{s}\left(t-\tau_{r}^{s}\right)\|-\| \boldsymbol{x}_{q}(t)-\boldsymbol{x}^{s}\left(t-\tau_{q}^{s}\right) \| \\
& +c\left(d t_{r q}(t)+\delta_{r q, f}(t)\right)-I_{r q, f}^{s}(t)+T_{r q}^{s}(t)+\lambda_{f}\left(\varphi_{r q, f}\left(t_{0}\right)+N_{r q, f}^{s}\right)+\epsilon_{r q, f}^{s}(t) .
\end{aligned}
$$

The initial satellite phases $\varphi_{, f}^{s}\left(t_{0}\right)$ are truly canceled by taking the differences.

In the above equations it has been assumed that the measurements at both receivers are performed strictly simultaneously, which is only true if the receiver clock errors of both receivers are identical. Standard GNSS receivers usually steer their receiver clock such that it is within $0.5 \mathrm{~ms}$ from system time, i.e., the time difference between the measurements is up to $1 \mathrm{~ms}$. This does not affect the assumptions made about satellite clock and instrumental delays.

\subsubsection{Between Satellite Differences}

The difference of the simultaneous code measurements of a receiver of the signals transmitted by two satellites follows from (2.1) as

$$
\begin{aligned}
p_{r, f}^{s}(t)-p_{r, f}^{k}(t)=\| & \boldsymbol{x}_{r}(t)-\boldsymbol{x}^{s}\left(t-\tau_{r}^{s}\right)\|-\| \boldsymbol{x}_{r}(t)-\boldsymbol{x}^{k}\left(t-\tau_{r}^{k}\right) \| \\
& +c\left(d t_{r}(t)-d t^{s}\left(t-\tau_{r}^{s}\right)+d_{r, f}(t)-d_{, f}^{s}\left(t-\tau_{r}^{s}\right)\right) \\
& -c\left(d t_{r}(t)-d t^{k}\left(t-\tau_{q}^{k}\right)+d_{r, f}(t)-d_{, f}^{k}\left(t-\tau_{r}^{k}\right)\right) \\
& +I_{r, f}^{s}(t)-I_{r, f}^{k}(t)+T_{r}^{s}(t)-T_{r}^{k}(t)+\varepsilon_{r, f}^{s}(t)-\varepsilon_{r, f}^{k}(t) .
\end{aligned}
$$

If the measurements corresponding to the satellites $s$ and $k$ are performed truly simultaneously, the receiver clock offset $d t_{r}(t)$ and instrumental delays $d_{r, f}(t)$ cancel exactly. With the abbreviation $(\cdot)^{s}-(\cdot)^{k}=(\cdot)^{s k}$, the between satellite differenced code observation can then be rewritten as

$$
\begin{aligned}
p_{r, f}^{s k}(t)=\left\|\boldsymbol{x}_{r}(t)-\boldsymbol{x}^{s}\left(t-\tau_{r}^{s}\right)\right\|-\left\|\boldsymbol{x}_{r}(t)-\boldsymbol{x}^{k}\left(t-\tau_{r}^{k}\right)\right\| \\
\quad-c\left(d t^{s k}\left(t-\tau_{r}^{s / k}\right)+d_{, f}^{s k}\left(t-\tau_{r}^{s / k}\right)\right)+I_{r, f}^{s k}(t)+T_{r}^{s k}(t)+\varepsilon_{r, f}^{s k}(t) .
\end{aligned}
$$

The notation $\tau_{r}^{s / k}$ is used to indicate the different travel times for the two satellites. The corresponding expression for the between satellite differenced carrier-phase observations reads (cf. $(2.2))$

$$
\begin{aligned}
\phi_{r, f}^{s k}(t)=\| & \boldsymbol{x}_{r}(t)-\boldsymbol{x}^{s}\left(t-\tau_{r}^{s}\right)\|-\| \boldsymbol{x}_{r}(t)-\boldsymbol{x}^{k}\left(t-\tau_{r}^{k}\right) \| \\
& -c\left(d t^{s k}\left(t-\tau_{r}^{s / k}\right)+\delta_{, f}^{s k}\left(t-\tau_{r}^{s / k}\right)\right)-I_{r, f}^{s k}(t)+T_{r}^{s k}(t) \\
& +\lambda_{f}\left(-\varphi_{, f}^{s k}\left(t_{0}\right)+N_{r, f}^{s k}\right)+\epsilon_{r, f}^{s k}(t) .
\end{aligned}
$$

The inital receiver phases $\varphi_{r, f}\left(t_{0}\right)$ are canceled by taking the differences. 


\subsubsection{Between Receiver and Satellite Differences}

The difference of the simultaneous between receiver code differences corresponding to two different satellites follow from (2.8) as

$$
\begin{aligned}
p_{r q, f}^{s}(t)-p_{r q, f}^{k}(t)=\| & \boldsymbol{x}_{r}(t)-\boldsymbol{x}^{s}\left(t-\tau_{r}^{s}\right)\|-\| \boldsymbol{x}_{q}(t)-\boldsymbol{x}^{s}\left(t-\tau_{q}^{s}\right) \| \\
& -\left\|\boldsymbol{x}_{r}(t)-\boldsymbol{x}^{k}\left(t-\tau_{r}^{k}\right)\right\|+\left\|\boldsymbol{x}_{q}(t)-\boldsymbol{x}^{k}\left(t-\tau_{q}^{k}\right)\right\| \\
& +c\left(d t_{r q}(t)+d_{r q, f}(t)\right)-c\left(d t_{r q}(t)+d_{r q, f}(t)\right) \\
& +c\left(d t_{r q}(t)+d_{r q, f}(t)\right)-c\left(d t_{r q}(t)+d_{r q, f}(t)\right) \\
& +I_{r q, f}^{s}(t)-I_{r q, f}^{k}(t)+T_{r q}^{s}(t)-T_{r q}^{k}(t)+\varepsilon_{r q, f}^{s}(t)-\varepsilon_{r q, f}^{k}(t) .
\end{aligned}
$$

The differenced receiver clock offsets and instrumental delays are now also canceled and the socalled double difference code observation can be finally written as

$$
\begin{aligned}
p_{r q, f}^{s k}(t)=\| & \boldsymbol{x}_{r}(t)-\boldsymbol{x}^{s}\left(t-\tau_{r}^{s}\right)\|-\| \boldsymbol{x}_{q}(t)-\boldsymbol{x}^{s}\left(t-\tau_{q}^{s}\right) \| \\
& -\left\|\boldsymbol{x}_{r}(t)-\boldsymbol{x}^{k}\left(t-\tau_{r}^{k}\right)\right\|+\left\|\boldsymbol{x}_{q}(t)-\boldsymbol{x}^{k}\left(t-\tau_{q}^{k}\right)\right\| \\
& +I_{r q, f}^{s k}(t)+T_{r q}^{s k}(t)+\varepsilon_{r q, f}^{s k}(t) .
\end{aligned}
$$

The corresponding expression for the double difference carrier-phase observations is derived similarly from (2.9) as

$$
\begin{aligned}
\phi_{r q, f}^{s k}(t)=\left\|\boldsymbol{x}_{r}(t)-\boldsymbol{x}^{s}\left(t-\tau_{r}^{s}\right)\right\|-\left\|\boldsymbol{x}_{q}(t)-\boldsymbol{x}^{s}\left(t-\tau_{q}^{s}\right)\right\| \\
-\left\|\boldsymbol{x}_{r}(t)-\boldsymbol{x}^{k}\left(t-\tau_{r}^{k}\right)\right\|+\left\|\boldsymbol{x}_{q}(t)-\boldsymbol{x}^{k}\left(t-\tau_{q}^{k}\right)\right\| \\
-I_{r q, f}^{s k}(t)+T_{r q}^{s k}(t)+\lambda_{f} N_{r q, f}^{s k}+\varepsilon_{r q, f}^{s k}(t) .
\end{aligned}
$$

The double differencing cancels all initial phases and only leaves the integer ambiguities $N_{r q, f}^{s k}$.

\subsection{Functional Models}

The functional models used for GNSS positioning based on the above undifferenced, single differenced, and double differenced observations are derived below. The observation equations were shown to be non-linear functions of the receiver coordinates $\boldsymbol{x}_{r}(t)$. These coordinates are the primary parameters of interest in positioning applications. Since standard least-squares methods are used to (iteratively) solve the positioning models, see Section 2.4, the observation equations are linearized. Moreover, the resulting system of linearized equations is not always of full column rank, i.e., not all of the unknown parameters can be estimated separately. It is shown how these systems of equations can be transformed to full column rank through the application of S-system theory (Baarda 1973; Teunissen 1984), so that the receiver position can be estimated without biases. The resulting systems of linearized observation equations can be written in the form

$$
\boldsymbol{y}=\boldsymbol{A a}+\boldsymbol{B b}+\boldsymbol{\eta}
$$

where $\boldsymbol{y} \in \mathbb{R}^{q}$ contains the code and carrier-phase measurements, $\boldsymbol{a} \in \mathbb{Z}^{n}$ the carrier-phase integer ambiguities, and $\boldsymbol{b} \in \mathbb{R}^{p}$ the real valued unknowns. The design matrices are given by $\boldsymbol{A}$ and $\boldsymbol{B}$, and $\boldsymbol{\eta}$ is an additive noise component, whose properties are discussed in Section 2.3. The other components of model (2.16) are discussed in the following. 
The models are formulated for a single measurement epoch and two GNSS receivers. The time stamps are thus omitted from now on for the sake of notational brevity. The models can be extended to multiple epochs by considering the time behavior of the parameters. Certain parameters like the carrier-phase ambiguities are time constant, whereas new parameters have to be introduced in each epoch for the time varying coordinates of a moving receiver. If known, more elaborate dynamic models linking the unknown parameters from consecutive epochs can be applied when combining multiple epochs of measurements. For the extension to more than two GNSS receivers, the reader is referred to, e.g., de Jonge (1998) or Odijk (2002). With a single GNSS receiver and no external information in form of bias corrections, it is not possible to separate the carrierphase integer ambiguities from the initial phases and instrumental delays of the satellites in a least-squares adjustment, so that integer ambiguity resolution is not feasible. Such single receiver models are thus not discussed in this dissertation.

\subsubsection{Undifferenced Model}

The undifferenced observation equations (2.1) and (2.2) are linearized with respect to the receiver coordinates $\boldsymbol{x}_{r}$. Let the approximate value of the receiver to satellite range $\rho_{r}^{s}$ be given by

$$
\rho_{r}^{s, 0}=\left\|\boldsymbol{x}_{r}^{0}-\boldsymbol{x}^{s, 0}\right\|
$$

where $\boldsymbol{x}_{r}^{0}$ and $\boldsymbol{x}^{s, 0}$ are the approximate receiver and satellite coordinates that can be extracted from a simple SPP solution. We note that the receiver position $\boldsymbol{x}_{r}^{0}$ refers to time $t$, whereas the satellite position $\boldsymbol{x}^{s, 0}$ refers to time $t-\tau_{r}^{s}$. The error of $\boldsymbol{x}^{s, 0}$ is caused by the uncertainty of both the description of the satellite orbit and the transmission time (both $t$ and $\tau_{r}^{s}$ are not known). The approximate values for all other parameters are implicitly assumed as zero, so that the observed minus computed code and carrier-phase measurements are given by

$$
\begin{aligned}
& \Delta p_{r, f}^{s}=p_{r, f}^{s}-\rho_{r}^{s, 0} \\
& \Delta \phi_{r, f}^{s}=\phi_{r, f}^{s}-\rho_{r}^{s, 0} .
\end{aligned}
$$

With the incremental receiver and satellite coordinates $\Delta \boldsymbol{x}_{r}=\boldsymbol{x}_{r}-\boldsymbol{x}_{r}^{0}$ and $\Delta \boldsymbol{x}^{s}=\boldsymbol{x}^{s}-\boldsymbol{x}^{s, 0}$, the linearization of the range increment $\Delta \rho_{r}^{s}=\rho_{r}^{s}-\rho_{r}^{s, 0}$ with respect to $\boldsymbol{x}_{r}$ and $\boldsymbol{x}^{s}$ is given by

$$
\Delta \rho_{r}^{s} \approx \boldsymbol{u}_{r}^{s, 0, \mathrm{~T}} \Delta \boldsymbol{x}_{r}-\boldsymbol{u}_{r}^{s, 0, \mathrm{~T}} \Delta \boldsymbol{x}^{s}
$$

The coefficient vector is the approximate unit line of sight vector pointing from satellite $s$ to receiver $r$

$$
\boldsymbol{u}_{r}^{s, 0}=\frac{\boldsymbol{x}_{r}^{0}-\boldsymbol{x}^{s, 0}}{\left\|\boldsymbol{x}_{r}^{0}-\boldsymbol{x}^{s, 0}\right\|}
$$

that is computed from the approximate receiver and satellite coordinates. The linearized observation equations now read

$$
\begin{aligned}
\Delta p_{r, f}^{s}= & \boldsymbol{u}_{r}^{s, 0, \mathrm{~T}} \Delta \boldsymbol{x}_{r}-\boldsymbol{u}_{r}^{s, 0, \mathrm{~T}} \Delta \boldsymbol{x}^{s}+c\left(d t_{r}-d t^{s}+d_{r, f}-d_{, f}^{s}\right)+I_{r, f}^{s}+T_{r}^{s}+\varepsilon_{r, f}^{s} \\
\Delta \phi_{r, f}^{s,}= & \boldsymbol{u}_{r}^{s, 0, \mathrm{~T}} \Delta \boldsymbol{x}_{r}-\boldsymbol{u}_{r}^{s, 0, \mathrm{~T}} \Delta \boldsymbol{x}^{s}+c\left(d t_{r}-d t^{s}+\delta_{r, f}-\delta_{, f}^{s}\right)-I_{r, f}^{s}+T_{r}^{s} \\
& \quad+\lambda_{f}\left(\varphi_{r, f}-\varphi_{, f}^{s}+N_{r, f}^{s}\right)+\epsilon_{r, f}^{s} .
\end{aligned}
$$

The incremental receiver and satellite coordinates are assumed sufficiently small so that the error due to the linearization is negligible. If the quality of the approximate values of the coordinates 
is too poor, an iterative procedure is necessary when solving the positioning problem, i.e., the linearization has to be repeated around updated values of the coordinates. The approximate satellite coordinates can be computed using either the broadcast ephemerides from the satellite's navigation message or the much more precise satellite orbit products from providers such as the International GNSS Service (IGS).

Let $S$ denote the number of satellites that are tracked by the two receivers $r=1$ and $r=2$ on $F$ frequencies. It is assumed that the same set of satellites is tracked by both receivers. The measurement vector $\boldsymbol{y}$ is defined as

$$
\boldsymbol{y}=\left[\boldsymbol{p}_{1}^{\mathrm{T}}, \boldsymbol{\phi}_{1}^{\mathrm{T}}, \boldsymbol{p}_{2}^{\mathrm{T}}, \boldsymbol{\phi}_{2}^{\mathrm{T}}\right]^{\mathrm{T}},
$$

with the receiver specific $F S$ vectors

$$
\begin{aligned}
& \boldsymbol{p}_{r}=\left[\Delta p_{r, 1}^{1}, \ldots, \Delta p_{r, 1}^{S}, \ldots, \Delta p_{r, F}^{1}, \ldots, \Delta p_{r, F}^{S}\right]^{\mathrm{T}} \\
& \boldsymbol{\phi}_{r}=\left[\Delta \phi_{r, 1}^{1}, \ldots, \Delta \phi_{r, 1}^{S}, \ldots, \Delta \phi_{r, F}^{1}, \ldots, \Delta \phi_{r, F}^{S}\right]^{\mathrm{T}}
\end{aligned}
$$

containing the observed minus computed code and carrier-phase measurements. We assume that the approximate satellite coordinates $\boldsymbol{x}^{s, 0}$ are sufficiently accurate, so that the residual satellite position errors can be neglected in a differential setup. The incremental satellite coordinates $\Delta \boldsymbol{x}^{s}$ are therefore not parameterized in the system model. The first order ionospheric approximation $I_{r, f}^{s}=\mu_{f} I_{r}^{s}$, cf. (2.4), and the tropospheric parameterization $T_{r}^{s}=\psi_{r}^{s} T_{\mathrm{z}, r}$, cf. (2.5), are applied. The preliminary vector of unknowns is then given by

$$
\tilde{\boldsymbol{b}}=\left[\Delta \boldsymbol{x}_{1}^{\mathrm{T}}, \Delta \boldsymbol{x}_{2}^{\mathrm{T}}, \boldsymbol{d}_{\mathrm{R}}^{\mathrm{T}}, \boldsymbol{d}_{\mathrm{S}}^{\mathrm{T}}, \iota^{\mathrm{T}}, T_{\mathrm{z}, 1}, T_{\mathrm{z}, 2}, \boldsymbol{m}^{\mathrm{T}}\right]^{\mathrm{T}},
$$

where the vectors of receiver clock and instrumental biases $\boldsymbol{d}_{\mathrm{R}}$, satellite clock and instrumental biases $\boldsymbol{d}_{\mathrm{S}}$, ionospheric delays $\boldsymbol{\iota}$, and carrier-phase ambiguities $\boldsymbol{m}$ are defined as

$$
\begin{aligned}
\boldsymbol{d}_{\mathrm{R}} & =c \cdot\left[d t_{1}, d_{1,1}, \ldots, d_{1, F}, \delta_{1,1}, \ldots, \delta_{1, F}, d t_{2}, d_{2,1}, \ldots, d_{2, F}, \delta_{2,1}, \ldots, \delta_{2, F}\right]^{\mathrm{T}} \\
\boldsymbol{d}_{\mathrm{S}} & =c \cdot\left[d t^{1}, \ldots, d t^{S}, d_{, 1}^{1}, \ldots, d_{, 1}^{S}, \ldots, d_{, F}^{1}, \ldots, d_{, F}^{S}, \delta_{, 1}^{1}, \ldots, \delta_{, 1}^{S}, \ldots, \delta_{, F}^{1}, \ldots, \delta_{, F}^{S}\right]^{\mathrm{T}} \\
\boldsymbol{\iota} & =\left[I_{1}^{1}, \ldots, I_{1}^{S}, I_{2}^{1}, \ldots, I_{2}^{S}\right]^{\mathrm{T}} \\
\boldsymbol{m} & =\left[M_{1,1}^{1}, \ldots, M_{1,1}^{S}, \ldots, M_{1, F}^{1}, \ldots, M_{1, F}^{S}, M_{2,1}^{1}, \ldots, M_{2,1}^{S}, \ldots, M_{2, F}^{1}, \ldots, M_{2, F}^{S}\right]^{\mathrm{T}} .
\end{aligned}
$$

The ordering in (2.26) is chosen so as to facilitate formulating the system matrix. Let $\tilde{\boldsymbol{B}}$ denote the preliminary system matrix corresponding to $\tilde{b}$, so that the system model (2.16) can also be written as $\boldsymbol{y}=\tilde{\boldsymbol{B}} \tilde{\boldsymbol{b}}+\boldsymbol{\eta}$. Note that so far there are no integer parameters. With $\otimes$ denoting the matrix Kronecker product, $\boldsymbol{e}_{k}$ the $k$-vector of ones, and $\boldsymbol{I}_{k}$ the unit matrix of size $k$, the partial design matrices of $\tilde{B}$ corresponding to the parameters in (2.25) for the measurement vector as defined in (2.23) are given by

$$
\begin{aligned}
& \text { blkdiag }\left(\boldsymbol{e}_{2 F} \otimes \boldsymbol{G}_{1}, \boldsymbol{e}_{2 F} \otimes \boldsymbol{G}_{2}\right) \\
& \boldsymbol{I}_{2} \otimes\left[\boldsymbol{e}_{2 F S}, \boldsymbol{I}_{2 F} \otimes \boldsymbol{e}_{S}\right] \\
& \boldsymbol{e}_{2} \otimes\left[\boldsymbol{e}_{2 F} \otimes-\boldsymbol{I}_{S},-\boldsymbol{I}_{2 F S}\right] \\
& \boldsymbol{I}_{2} \otimes\left[\begin{array}{c}
\boldsymbol{\mu} \\
-\boldsymbol{\mu}
\end{array}\right] \otimes \boldsymbol{I}_{S} \\
& \text { blkdiag }\left(\boldsymbol{\psi}_{1}, \boldsymbol{\psi}_{2}\right) \\
& \boldsymbol{I}_{2} \otimes\left[\begin{array}{c}
\mathbf{0}_{F \times F} \\
\boldsymbol{\Lambda}
\end{array}\right] \otimes \boldsymbol{I}_{S}
\end{aligned}
$$$$
\text { receiver coordinates }
$$$$
\text { receiver clock and instrumental delays }
$$$$
\text { satellite clock and instrumental delays }
$$$$
\text { ionospheric delays }
$$$$
\text { tropospheric zenith delays }
$$$$
\text { non-integer ambiguities. }
$$ 
The geometry matrices $\boldsymbol{G}_{r} \in \mathbb{R}^{S \times 3}$ contain the satellite to receiver unit vectors

$$
\boldsymbol{G}_{r}=\left[\begin{array}{c}
\boldsymbol{u}_{r}^{1,0, \mathrm{~T}} \\
\vdots \\
\boldsymbol{u}_{r}^{S, 0, \mathrm{~T}}
\end{array}\right]
$$

the $F$-vector $\boldsymbol{\mu}=\left[\mu_{1}, \ldots, \mu_{F}\right]^{\mathrm{T}}$ the coefficients of the first order ionospheric delay, the $S$-vector $\boldsymbol{\psi}_{r}=\left[\psi_{r}^{1}, \ldots, \psi_{r}^{S}\right]^{\mathrm{T}}$ the values of the tropospheric mapping function at receiver $r$, and $\boldsymbol{\Lambda}$ is a diagonal matrix with the $F$ wavelengths $\lambda_{f}$ as its entries

$$
\boldsymbol{\Lambda}=\left[\begin{array}{ccc}
\lambda_{1} & & \\
& \ddots & \\
& & \lambda_{F}
\end{array}\right]
$$

Since the design matrix $\tilde{\boldsymbol{B}}$ is rank deficient, it is not possible to formulate an unbiased estimator for the parameter vector $\tilde{\boldsymbol{b}}$. By properly transforming the parameter vector, i.e., by forming combinations of the original parameters, a full rank system of equations can be established and subsequently solved. The theory for such transformations is given by the S-basis techniques, of which a summary is presented in Section A1. One specific way of how the rank deficiencies of the above system model can be resolved through application of S-system theory is now demonstrated. One can think of infinitely many other ways to do so, which means that a different S-system is chosen and different parameter combinations are formed.

Receiver and Satellite Clock and Instrumental Biases The rank deficiencies are resolved one after another, so that we only consider certain subsets of the parameter vector $\tilde{b}$ and the respective columns of the design matrix in each step. The transformation is shown in detail for the clock offset $c d t_{1}$ and the instrumental delays $c d_{1, f}$ and $c \delta_{1, f}$ of the first receiver. The corresponding $4 F S \times(1+2 F)$ partial design matrix and parameter vector read

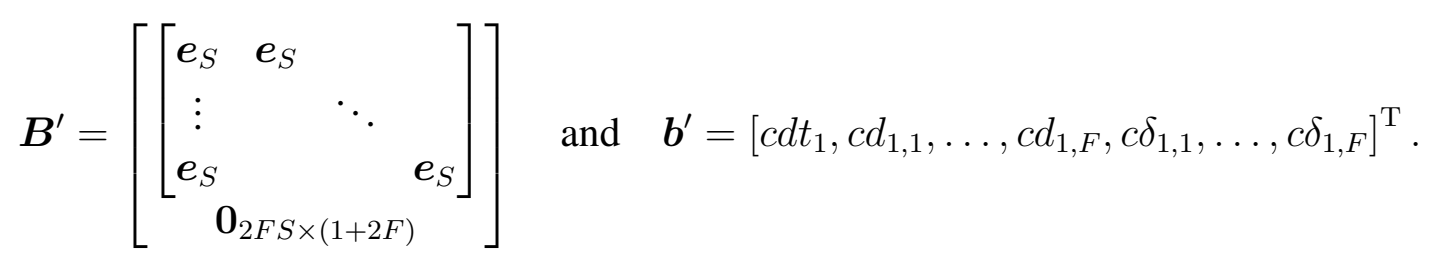

Matrix $\boldsymbol{B}^{\prime}$ has a rank deficiency of size one, i.e., the effect of a change of the receiver clock on the measurements is canceled by a shift of the code and carrier-phase instrumental delays on all frequencies with opposite sign and identical magnitude. It is therefore not possible to estimate both the absolute receiver clock offset and the instrumental delays. In order to obtain a full rank system, one constraint in the form $\boldsymbol{S}^{\perp, \mathrm{T}} \boldsymbol{b}^{\prime}$, referred to as $\mathrm{S}$-basis, has to be added. The basis of the null space $\mathcal{N}\left(\boldsymbol{B}^{\prime}\right)$ can be chosen as the $(1+F S) \times 1$ matrix $\boldsymbol{V}=[-1,1, \ldots, 1]^{\mathrm{T}}$. We may now choose $\boldsymbol{S}^{\perp}$ as, e.g.,

$$
\boldsymbol{S}^{\perp}=[1,0, \ldots, 0]^{\mathrm{T}} .
$$

That is, the receiver clock offset $c d t_{1}$ is chosen as S-basis element. With this choice of $\boldsymbol{S}^{\perp}$ the condition $\mathcal{R}(\boldsymbol{S})+\mathcal{N}\left(\boldsymbol{B}^{\prime}\right)=\mathbb{R}^{1+2 F}$ is fulfilled, where $\mathcal{R}(\cdot)$ denotes the range space of a matrix and 
$\boldsymbol{S}$ contains basis vectors of the space orthogonal to $\mathcal{R}\left(\boldsymbol{S}^{\perp}\right)$. We can now choose the $(1+F S) \times F S$ matrix $\boldsymbol{S}$ defining the $\mathrm{S}$-system as, e.g.,

$$
\boldsymbol{S}=\left[\begin{array}{c}
\mathbf{0}_{1 \times F S} \\
\boldsymbol{I}_{F S}
\end{array}\right]
$$

A matrix with basis vectors for the orthogonal complement of $\mathcal{N}\left(\boldsymbol{B}^{\prime}\right)=\mathcal{R}(\boldsymbol{V})$ is given by

$$
\boldsymbol{V}^{\perp}=\left[\begin{array}{c}
\boldsymbol{e}_{F S}^{\mathrm{T}} \\
\boldsymbol{I}_{F S}
\end{array}\right]
$$

The vector $\boldsymbol{\alpha}$ of unbiased estimable parameters for the S-system of $\boldsymbol{S}$ follows from (A6) as

$$
\boldsymbol{\alpha}=\left[\boldsymbol{V}^{\perp, \mathrm{T}} \boldsymbol{S}\right]^{-1} \boldsymbol{V}^{\perp, \mathrm{T}} \boldsymbol{b}^{\prime}=\boldsymbol{V}^{\perp, \mathrm{T}} \boldsymbol{b}^{\prime}
$$

With the definition of $\boldsymbol{b}^{\prime}$ in (2.30) the estimable parameter functions are

$$
c d t_{1, f}=c d t_{1}+c d_{1, f} \quad \text { and } \quad c \delta t_{1, f}=c d t_{1}+c \delta_{1, f}
$$

i.e., the receiver clock offset is lumped with the instrumental delays. The design matrix for the new parameter vector $\boldsymbol{\alpha}$ is $\boldsymbol{B}^{\prime} \boldsymbol{S}$, cf. (A5), i.e., the first column of $\boldsymbol{B}^{\prime}$ is removed. It is easily verified that this transformation does not change the observation model. The same transformation is applied to the receiver clock and instrumental biases of the second receiver.

Similarly, the rank deficiency of size $S$ resulting from the satellite clock offsets $c d t^{s}$ and instrumental delays $c d_{, f}^{s}$ and $c \delta_{, f}^{s}$ can be resolved by introducing the lumped parameters

$$
c d t_{, f}^{s}=c d t^{s}+c d_{, f}^{s} \quad \text { and } \quad c \delta t_{, f}^{s}=c d t^{s}+\delta_{, f}^{s} .
$$

After the parameter transformations (2.35) and (2.36), there is still a rank deficiency of size $2 F$ when considering both the receiver and satellite clock parameters. We choose the $2 F$ combined receiver clock and instrumental offsets $c d t_{1, f}$ and $c \delta t_{1, f}$ of the first receiver as S-basis elements and define the relative clock errors

$$
\begin{aligned}
\boldsymbol{d}_{\mathrm{R}}=c \cdot & {\left[d t_{21,1}, \ldots, d t_{21, F}, \delta t_{21,1}, \ldots, \delta t_{21, F}\right]^{\mathrm{T}} } \\
\boldsymbol{d}_{\mathrm{S}}=c \cdot & {\left[d t_{, 1}^{1}-d t_{1,1}, \ldots, d t_{, 1}^{S}-d t_{1,1}, \ldots, d t_{, F}^{1}-d t_{1, F}, \ldots, d t_{, F}^{S}-d t_{1, F},\right.} \\
& \left.\delta t_{, 1}^{1}-\delta t_{1,1}, \ldots, \delta t_{, 1}^{S}-\delta t_{1,1}, \ldots, \delta t_{, F}^{1}-\delta t_{1, F}, \ldots, \delta t_{, F}^{S}-\delta t_{1, F}\right]^{\mathrm{T}},
\end{aligned}
$$

where $(\cdot)_{21}=(\cdot)_{2}-(\cdot)_{1}$. The design matrices, which are now of full rank when combined, are given by

$$
\begin{array}{ll}
{\left[\begin{array}{c}
\mathbf{0}_{2 F S \times 2 F} \\
\boldsymbol{I}_{2 F} \otimes \boldsymbol{e}_{S}
\end{array}\right]} & \text { receiver clock and instrumental delays } \\
\boldsymbol{e}_{2} \otimes-\boldsymbol{I}_{2 F S} & \text { satellite clock and instrumental delays. }
\end{array}
$$

Note that the vector $\boldsymbol{d}_{\mathrm{S}}$ of satellite clock errors also contains the clock biases of the first receiver. 
Satellite Phase Clock Offsets and Ambiguities Another rank deficiency of size $F S$ is identified for the design matrix columns of the re-parameterized satellite clock offsets (2.37) and the non-integer carrier-phase ambiguities $\boldsymbol{m}$. This can be resolved by combining the carrier-phase ambiguity parameters $M_{1, f}^{s}$ of the first receiver with the satellite clock biases, and by replacing the undifferenced ambiguities with the between receiver differenced ambiguities:

$$
\begin{gathered}
\boldsymbol{d}_{\mathrm{S}}=\left[\ldots, c \delta t_{, 1}^{1}-c \delta t_{1,1}-\lambda_{1} M_{1,1}^{1}, \ldots, c \delta t_{, 1}^{S}-c \delta t_{1,1}-\lambda_{1} M_{1,1}^{S}, \ldots\right. \\
\left.\ldots, c \delta t_{, F}^{1}-c \delta t_{1, F}-\lambda_{F} M_{1, F}^{1}, \ldots, c \delta t_{, F}^{S}-c \delta t_{1, F}-\lambda_{F} M_{1, F}^{S}\right]^{\mathrm{T}} \\
\boldsymbol{m}=\left[M_{21,1}^{1}, \ldots, M_{21,1}^{S}, \ldots, M_{21, F}^{1}, \ldots, M_{21, F}^{S}\right]^{\mathrm{T}}
\end{gathered}
$$

Note that the first part of the satellite clock bias vector $\boldsymbol{d}_{\mathrm{S}}$ containing the code biases remains unchanged and is not written down explicitly. With the design matrix of the satellite clock bias parameters from (2.38), the ambiguity design matrix reads:

$$
\left[\begin{array}{c}
\mathbf{0}_{3 F S \times F S} \\
\boldsymbol{\Lambda} \otimes \boldsymbol{I}_{S}
\end{array}\right] \quad \text { non-integer ambiguities }
$$

Receiver Phase Clock Offsets and Ambiguities Further, the partial design matrices (2.40) and (2.38) of the between receiver differenced ambiguities and the between receiver differenced phase clock offsets show a rank deficiency of size $F$. We may choose the $F$ between receiver differenced ambiguities of the first satellite as S-basis elements. With a proper choice of the S-system matrix $\boldsymbol{S}$, we get the parameter vectors

$$
\begin{aligned}
\boldsymbol{d}_{\mathrm{R}} & =\left[\ldots, c \delta t_{21,1}+\lambda_{1} M_{21,1}^{1}, \ldots, c \delta t_{21, F}+\lambda_{F} M_{21, F}^{1}\right]^{\mathrm{T}} \\
\boldsymbol{m} & =\left[M_{21,1}^{21}, \ldots, M_{21,1}^{S 1}, \ldots, M_{21, F}^{21}, \ldots, M_{21, F}^{S 1}\right]^{\mathrm{T}}
\end{aligned}
$$

where the receiver clock code biases contained in $\boldsymbol{d}_{\mathrm{R}}$ are again not written down explicitly. The double difference ambiguities $M_{21, f}^{s 1}$ are given by

$$
\begin{aligned}
M_{21, f}^{s 1} & =\left(\varphi_{2, f}-\varphi_{, f}^{s}+N_{2, f}^{s}-\varphi_{2, f}-\varphi_{, f}^{1}+N_{2, f}^{1}\right)-\left(\varphi_{1, f}-\varphi_{, f}^{s}+N_{1, f}^{s}-\varphi_{1, f}-\varphi_{, f}^{1}+N_{1, f}^{1}\right) \\
& =N_{2, f}^{s}-N_{2, f}^{1}-N_{1, f}^{s}+N_{1, f}^{1}=N_{21}^{s 1},
\end{aligned}
$$

i.e,. they are integers. The design matrix of the receiver clock bias vector remains unchanged, and the ambiguity design matrix according to (2.41) is now

$$
\left[\begin{array}{c}
\mathbf{0}_{3 F S \times F(S-1)} \\
\Lambda \otimes\left[\begin{array}{c}
\mathbf{0}_{1 \times(S-1)} \\
\boldsymbol{I}_{S-1}
\end{array}\right]
\end{array}\right] \quad \text { integer ambiguities. }
$$

Receiver Coordinates, Tropospheric Delays, and Satellite Clock Offsets The between receiver distances for the positioning applications discussed in this dissertation are small (at most a few hundred kilometers) compared to the ranges from the satellites to the receivers. That is, the satellite to receiver unit vectors are approximately parallel for both receivers and $\boldsymbol{G}_{1} \approx \boldsymbol{G}_{2}$. This means that a near rank deficiency of size 3 is present in the combined design matrix of the receiver coordinates and the satellite clock biases, which becomes a real rank deficiency for $\boldsymbol{G}_{1}=\boldsymbol{G}_{2}$. Typically, one of the two receivers, say $r=1$, is used as a reference station, whose coordinates are 
precisely known. In that case $\Delta \boldsymbol{x}_{1}=\mathbf{0}$ and we can simply remove the incremental receiver coordinates $\Delta \boldsymbol{x}_{1}$ from the system model, which also removes the rank deficiency. The design matrix for the receiver coordinates of the second receiver is

$$
\left[\begin{array}{c}
\mathbf{0}_{2 F S \times 3} \\
\boldsymbol{e}_{2 F} \otimes \boldsymbol{G}_{2}
\end{array}\right] \quad \text { receiver coordinates. }
$$

The exact same problem occurs between the two tropospheric zenith delays and their respective vectors $\psi_{1}$ and $\psi_{2}$ containing the values of the mapping function. For short between receiver distances, the mapping functions produce nearly identical values, which for $\boldsymbol{\psi}_{1}=\boldsymbol{\psi}_{2}$ results in a true rank deficiency of size one with the satellite clock biases. This can be resolved by absorbing the tropospheric delays at the first receiver in the satellite clock offsets, i.e., by choosing $T_{z, 1}$ as S-basis element:

$$
\begin{aligned}
\boldsymbol{d}_{\mathrm{S}}=[ & c d t_{, 1}^{1}-c d t_{1,1}-\psi_{1}^{1} T_{\mathrm{z}, 1}, \ldots, c d t_{, 1}^{S}-c d t_{1,1}-\psi_{1}^{S} T_{\mathrm{z}, 1}, \ldots \\
& \ldots c d t_{, F}^{1}-c d t_{1, F}-\psi_{1}^{1} T_{\mathrm{z}, 1}, \ldots, c d t_{, F}^{S}-c d t_{1, F}-\psi_{1}^{S} T_{\mathrm{z}, 1} \\
& c \delta t_{, 1}^{1}-c \delta t_{1,1}-\lambda_{1} M_{1,1}^{1}-\psi_{1}^{1} T_{\mathrm{z}, 1}, \ldots, c \delta t_{, 1}^{S}-c \delta t_{1,1}-\lambda_{1} M_{1,1}^{S}-\psi_{1}^{S} T_{\mathrm{z}, 1}, \ldots \\
& \left.\ldots, c \delta t_{, F}^{1}-c \delta t_{1, F}-\lambda_{F} M_{1, F}^{1}-\psi_{1}^{1} T_{\mathrm{z}, 1}, \ldots, c \delta t_{, F}^{S}-c \delta t_{1, F}-\lambda_{F} M_{1, F}^{S}-\psi_{1}^{S} T_{\mathrm{z}, 1}\right]^{\mathrm{T}}
\end{aligned}
$$

The remaining tropospheric zenith delay parameter is $T_{z, 21}=T_{z, 2}-T_{z, 1}$, i.e., the relative tropospheric zenith delay can be estimated. The corresponding part of the design matrix reduces to the column vector

$$
\left[\begin{array}{c}
\mathbf{0}_{2 F S \times 1} \\
\boldsymbol{e}_{2 F} \otimes \boldsymbol{\psi}_{2}
\end{array}\right] \quad \text { tropospheric zenith delay. }
$$

Note that possible uncertainties of the coordinates of the reference receiver are absorbed by the satellite clocks in the same way as the tropospheric zenith delay of this receiver, i.e., in fact the relative coordinates $\Delta \boldsymbol{x}_{21}=\Delta \boldsymbol{x}_{2}-\Delta \boldsymbol{x}_{1}$ are estimable.

Ionospheric Delays, Satellite Clock Offsets, and Receiver Clock Offsets A last rank deficiency of size $S+1$ is present between the columns of the design matrix corresponding to the ionospheric slant delays $\iota$ and the satellite and receiver clock offsets $\boldsymbol{d}_{\mathrm{S}}$ and $\boldsymbol{d}_{\mathrm{R}}$. By lumping the ionospheric slant delays of the first receiver with the satellite clock offsets we can remove a rank deficiency of size $S$. The final satellite clock parameters are given by

$$
\boldsymbol{d}_{\mathrm{S}}=\left[c \tilde{d} t_{, 1}^{1}, \ldots, c \tilde{d} t_{, 1}^{S}, \ldots, c \tilde{d} t_{, F}^{1}, \ldots, c \tilde{d} t_{, F}^{S}, c \tilde{\delta} t_{, 1}^{1}, \ldots, c \tilde{\delta} t_{, 1}^{S}, \ldots, c \tilde{\delta} t_{, F}^{1}, \ldots, c \tilde{\delta} t_{, F}^{S}\right]^{\mathrm{T}}
$$

with

$$
\begin{aligned}
c \tilde{d}_{, f}^{s} & =c d t_{, f}^{s}-c d t_{1, f}-\psi_{1}^{s} T_{\mathrm{z}, 1}-\mu_{f} I_{1}^{s} \\
c \tilde{\delta} t_{, f}^{s} & =c \delta t_{, f}^{s}-c \delta t_{1, f}-\lambda_{f} M_{1, f}^{s}-\psi_{1}^{s} T_{\mathrm{z}, 1}+\mu_{f} I_{1}^{s} .
\end{aligned}
$$

The intermediate ionospheric parameters are the relative ionospheric slant delays $I_{21}^{s}=I_{2}^{s}-I_{1}^{s}$. The last rank deficiency of size one between these relative ionospheric delays and the receiver 
clock offsets is resolved by combining the receiver clock parameters with the between receiver differenced ionospheric delay of the first satellite. The final parameter vectors are then

$$
\begin{aligned}
\boldsymbol{d}_{\mathrm{R}} & =\left[\tilde{c} \tilde{d} t_{21,1}, \ldots, \tilde{c} \tilde{d} t_{21, F}, c \tilde{\delta} t_{21,1}, \ldots, c \tilde{\delta} t_{21, F}\right]^{\mathrm{T}} \\
\boldsymbol{\iota} & =\left[I_{21}^{21}, \ldots, I_{21}^{S 1}\right]^{\mathrm{T}}
\end{aligned}
$$

with

$$
\tilde{c} t_{21, f}=c d t_{21, f}+\mu_{f} I_{21}^{1}, \quad \text { and } \quad c \tilde{\delta} t_{21, f}=c \delta t_{21, f}+\lambda_{f} M_{21, f}^{1}-\mu_{f} I_{21}^{1} .
$$

It is noted that the estimable ionospheric parameters are the double differenced ionospheric delays on the first frequency. Their final partial design matrix is given by

$$
\left.\left[\begin{array}{c}
\mathbf{0}_{2 F S \times(S-1)} \\
\boldsymbol{\mu} \\
-\boldsymbol{\mu}
\end{array}\right] \otimes\left[\begin{array}{c}
\boldsymbol{0}_{1 \times(S-1)} \\
\boldsymbol{I}_{S-1}
\end{array}\right]\right] \quad \text { ionospheric delays. }
$$

The Full Rank Undifferenced Model The estimable parameters of the full rank linearized model (2.16) with undifferenced observations corresponding to the above employed S-system are given in Table 2.1. Note that the receiver and satellite clock offsets are in fact the lumped parameters as defined in (2.48) and (2.50).

Table 2.1: Parameters of full rank undifferenced model.

\begin{tabular}{lll}
\hline Parameter & Contains & Design matrix \\
\hline $\boldsymbol{y}$ & $2 F S$ code observations $\Delta p_{r, f}^{s}$ & - \\
& $2 F S$ carrier-phase observations $\Delta \phi_{r, f}^{s}$ & - \\
$\boldsymbol{a}$ & $F(S-1)$ double difference ambiguities $N_{21, f}^{s 1}$ & $(2.43)$ \\
$\boldsymbol{b}$ & 3 incremental receiver coordinates $\Delta \boldsymbol{x}_{2} / \Delta \boldsymbol{x}_{21}$ & $(2.44)$ \\
& $2 F$ receiver clock offsets $\tilde{c} t_{21, f}$ and $\tilde{\tilde{\delta}} t_{21, f}$ & $(2.38)$ \\
& $2 F S$ satellite clock offsets $c \tilde{d} t_{, f}^{s}$ and $c \tilde{\delta} t_{, f}^{s}$ & $(2.38)$ \\
& $S-1$ double difference ionospheric delays $I_{21}^{s 1}$ & $(2.51)$ \\
& 1 relative tropospheric zenith delay $T_{z, 21}$ & $(2.46)$ \\
\hline
\end{tabular}

\subsubsection{Single Difference Models}

The full rank functional positioning models for single difference observations, i.e., for either between receiver differenced or between satellite differenced observations, are presented.

The Full Rank Between Receiver Difference Model Using the linearization as introduced for the undifferenced observation equations in Section 2.2.1, the linearized version of the observed minus computed between receiver differenced code and carrier-phase observation equations (2.8) and (2.9) follows as

$$
\begin{aligned}
& \Delta p_{r q, f}^{s}= \boldsymbol{u}_{r}^{s, 0, \mathrm{~T}} \Delta \boldsymbol{x}_{r}-\boldsymbol{u}_{r}^{s, 0, \mathrm{~T}} \Delta \boldsymbol{x}^{s}-\boldsymbol{u}_{q}^{s, 0, \mathrm{~T}} \Delta \boldsymbol{x}_{q}+\boldsymbol{u}_{q}^{s, 0, \mathrm{~T}} \Delta \boldsymbol{x}^{s} \\
& \quad+c\left(d t_{r q}+d_{r q, f}\right)+I_{r q, f}^{s}+T_{r q}^{s}+\varepsilon_{r q, f}^{s} \\
& \Delta \phi_{r q, f}^{s}=\boldsymbol{u}_{r}^{s, 0, \mathrm{~T}} \Delta \boldsymbol{x}_{r}-\boldsymbol{u}_{r}^{s, 0, \mathrm{~T}} \Delta \boldsymbol{x}^{s}-\boldsymbol{u}_{q}^{s, 0, \mathrm{~T}} \Delta \boldsymbol{x}_{q}+\boldsymbol{u}_{q}^{s, 0, \mathrm{~T}} \Delta \boldsymbol{x}^{s} \\
& \quad+c\left(d t_{r q}+\delta_{r q, f}\right)-I_{r q, f}^{s}+T_{r q}^{s}+\lambda_{f}\left(\varphi_{r q, f}+N_{r q, f}^{s}\right)+\epsilon_{r q, f}^{s} .
\end{aligned}
$$


It is noted that the two $\Delta \boldsymbol{x}^{s}$ terms are not exactly identical, since they are based on satellite positions that are evaluated at slightly different time instances. With $S$ and $F$ the number of tracked satellites and used frequencies, the measurement vector $\boldsymbol{y}$ is defined as

$$
\boldsymbol{y}=\left[\boldsymbol{p}_{21}^{\mathrm{T}}, \boldsymbol{\phi}_{21}^{\mathrm{T}}\right]^{\mathrm{T}}
$$

with the $F S$ vectors

$$
\begin{aligned}
\boldsymbol{p}_{21} & =\left[\Delta p_{21,1}^{1}, \ldots, \Delta p_{21, F}^{S}, \ldots, \Delta p_{21, F}^{1}, \ldots, \Delta p_{21, F}^{S}\right]^{\mathrm{T}} \\
\boldsymbol{\phi}_{21} & =\left[\Delta \phi_{21,1}^{1}, \ldots, \Delta \phi_{21, F}^{S}, \ldots, \Delta \phi_{21, F}^{1}, \ldots, \Delta \phi_{21, F}^{S}\right]^{\mathrm{T}}
\end{aligned}
$$

We make the same assumptions as for the undifferenced model, namely that the satellite coordinates $\boldsymbol{x}^{s}$ are not parameterized and the between receiver distance is relatively small so that the values of the tropospheric mapping function $\psi_{1}^{s}$ and $\psi_{2}^{s}$ are identical. Although the differencing cancels the satellite clock and instrumental delays, the resulting positioning model is still rank deficient when considering all unknowns in (2.52) and (2.53) as separate parameters. We note that the vector of between receiver differenced observations (2.54) follows from the vector of undifferenced observations as defined in (2.23) through a linear transformation in form of the matrix multiplication with $\left[-\boldsymbol{I}_{2 F S}, \boldsymbol{I}_{2 F S}\right]$. We can thus start with the full rank undifferenced model and simply apply this transformation to the corresponding design matrices. The partial design matrices of the full rank between receiver differenced model follow as

$$
\begin{array}{ll}
\boldsymbol{e}_{2 F} \otimes \boldsymbol{G}_{2} & \text { receiver coordinates } \\
\boldsymbol{I}_{2 F} \otimes \boldsymbol{e}_{S} & \text { receiver clock and instrumental delays } \\
{\left[\begin{array}{c}
\boldsymbol{\mu} \\
-\boldsymbol{\mu}
\end{array}\right] \otimes\left[\begin{array}{c}
\mathbf{0}_{1 \times(S-1)} \\
\boldsymbol{I}_{S-1}
\end{array}\right]} & \text { ionospheric delays } \\
\boldsymbol{e}_{2 F} \otimes \boldsymbol{\psi}_{2} & \text { tropospheric zenith delay } \\
{\left[\begin{array}{c}
\mathbf{0}_{F S \times F(S-1)} \\
\boldsymbol{\Lambda} \otimes\left[\begin{array}{c}
\mathbf{0}_{1 \times(S-1)} \\
\boldsymbol{I}_{S-1}
\end{array}\right]
\end{array}\right]} & \text { integer ambiguities. }
\end{array}
$$

The design matrix of the satellite clock parameters is an all-zeros matrix, i.e., these parameters do not appear in the between receiver differenced model. This could be expected from the observation equations, in which the satellite specific delays are also canceled. The other estimable parameters are the same as in the undifferenced model.

The estimable parameters of the full rank linearized model (2.16) with between receiver differenced observations are given in Table 2.2. We can see that some of the parameters like the coordinates, the receiver clock offsets, and the tropospheric zenith delay appear in between receiver differenced form. That is, when defining the S-system for the undifferenced model, it has been implicitly chosen a between receiver differencing like parameter transformation.

The Full Rank Between Satellite Difference Model With the linearization introduced in Section 2.2.1 for undifferenced observations, the linearized observed minus computed between satel- 
Table 2.2: Parameters of full rank between receiver difference model; the design matrices are given in (2.56).

\begin{tabular}{ll}
\hline Parameter & Contains \\
\hline $\boldsymbol{y}$ & $F S$ code observations $\Delta p_{21, f}^{s}$ \\
& $F S$ carrier-phase observations $\Delta \phi_{21, f}^{s}$ \\
$\boldsymbol{a}$ & $F(S-1)$ double difference ambiguities $N_{21, f}^{s 1}$ \\
$\boldsymbol{b}$ & 3 incremental receiver coordinates $\Delta \boldsymbol{x}_{2} / \Delta \boldsymbol{x}_{21}$ \\
& $2 F$ receiver clock offsets $\tilde{d} t_{21, f}$ and $c \tilde{\delta} t_{21, f}$ \\
& $S-1$ double difference ionospheric delays $I_{21}^{s 1}$ \\
& 1 relative tropospheric zenith delay $T_{z, 21}$ \\
\hline
\end{tabular}

lite differenced code and carrier-phase observation equations (2.11) and (2.12) read

$$
\begin{gathered}
\Delta p_{r, f}^{s k}=\boldsymbol{u}_{r}^{s, \mathrm{~T}} \Delta \boldsymbol{x}_{r}-\boldsymbol{u}_{r}^{s, \mathrm{~T}} \Delta \boldsymbol{x}^{s}-\boldsymbol{u}_{r}^{k, \mathrm{~T}} \Delta \boldsymbol{x}_{r}+\boldsymbol{u}_{r}^{k, \mathrm{~T}} \Delta \boldsymbol{x}^{k} \\
\quad-c\left(d t^{s k}+d_{, f}^{s k}\right)+I_{r, f}^{s k}+T_{r}^{s k}+\varepsilon_{r, f}^{s k} \\
\begin{aligned}
\Delta \phi_{r, f}^{s k}= & \boldsymbol{u}_{r}^{s, \mathrm{~T}} \Delta \boldsymbol{x}_{r}-\boldsymbol{u}_{r}^{s, \mathrm{~T}} \Delta \boldsymbol{x}^{s}-\boldsymbol{u}_{r}^{k, \mathrm{~T}} \Delta \boldsymbol{x}_{r}+\boldsymbol{u}_{r}^{k, \mathrm{~T}} \Delta \boldsymbol{x}^{k} \\
& -c\left(d t^{s k}+\delta_{, f}^{s k}\right)-I_{r, f}^{s k}+T_{r}^{s k}+\lambda_{f}\left(-\varphi_{, f}^{s k}+N_{r, f}^{s k}\right)+\epsilon_{r, f}^{s k} .
\end{aligned}
\end{gathered}
$$

With $S$ and $F$ the number of satellites that are tracked by both receivers and the number of used frequencies, the measurement vector $\boldsymbol{y}$ is defined as

$$
\boldsymbol{y}=\left[\boldsymbol{p}_{1}^{1, \mathrm{~T}}, \boldsymbol{\phi}_{1}^{1, \mathrm{~T}}, \boldsymbol{p}_{2}^{1, \mathrm{~T}}, \boldsymbol{\phi}_{2}^{1, \mathrm{~T}}\right]^{\mathrm{T}}
$$

with the $F(S-1)$ vectors

$$
\begin{aligned}
\boldsymbol{p}_{r}^{1} & =\left[\Delta p_{r, 1}^{21}, \ldots, \Delta p_{r, 1}^{S 1}, \ldots, \Delta p_{r, F}^{21}, \ldots, \Delta p_{r, F}^{S 1}\right]^{\mathrm{T}} \\
\boldsymbol{\phi}_{r}^{1} & =\left[\Delta \phi_{r, 1}^{21}, \ldots, \Delta \phi_{r, 1}^{S 1}, \ldots, \Delta \phi_{r, F}^{21}, \ldots, \Delta \phi_{r, F}^{S 1}\right]^{\mathrm{T}} .
\end{aligned}
$$

The first satellite is chosen as pivot satellite and its measurements are subtracted from the ones of all other satellites. Again, the same assumptions are made as for the undifferenced model, i.e., the satellite coordinates $\boldsymbol{x}^{s}$ are not parameterized and the values of the tropospheric mapping function are identical for both receivers. The differencing cancels the receiver dependent clock parameters, but still results in a rank deficient system of equations. With $\boldsymbol{D}=\left[-\boldsymbol{e}_{S-1}, \boldsymbol{I}_{S-1}\right]$, the between satellite differenced measurement vector is computed from the vector of undifferenced observations via a transformation with the matrix $\left[\boldsymbol{I}_{4 F} \otimes \boldsymbol{D}\right]$. Applying this transformation to the design matrix of the full rank undifferenced model produces an all zeros design matrix for the receiver clock offsets, i.e., they are removed from the model. It still leaves a rank deficiency of size $2 F$ in the design matrix of the satellite clock offsets, which is given by

$$
\boldsymbol{e}_{2} \otimes \boldsymbol{I}_{2 F} \otimes-\boldsymbol{D} \quad \text { satellite clock and instrumental delays. }
$$

This rank deficiency is removed by subtracting the $2 F$ clock offsets corresponding to the pivot satellite from the other clock offsets. The satellite clock parameter vector is then given by

$$
\boldsymbol{d}_{\mathrm{S}}=\left[\tilde{c} \tilde{d} t_{, 1}^{21}, \ldots, c \tilde{d} t_{, 1}^{S 1}, \ldots, c \tilde{d} t_{, F}^{21}, \ldots, c \tilde{d} t_{, F}^{S 1}, c \tilde{\delta} t_{, 1}^{21}, \ldots, c \tilde{\delta} t_{, 1}^{S 1}, \ldots, c \tilde{\delta} t_{, F}^{21}, \ldots, c \tilde{\delta} t_{, F}^{S 1}\right]^{\mathrm{T}}
$$


with

$$
c \tilde{d} t_{, f}^{s 1}=c d t_{, f}^{s 1}-\psi_{1}^{s 1} T_{\mathrm{z}, 1}-\mu_{f} I_{1}^{s 1}, \quad \text { and } \quad c \tilde{\delta} t_{, f}^{s 1}=c \delta t_{, f}^{s 1}-\lambda_{f} M_{1, f}^{s 1}-\psi_{1}^{s 1} T_{\mathrm{z}, 1}+\mu_{f} I_{1}^{s 1}
$$

That is, the receiver clock terms cancel and the parameters appear in between satellite differenced form like in the observation equations (2.57) and (2.58). The design matrices of the full rank between satellite difference model are

$$
\begin{array}{ll}
{\left[\begin{array}{c}
\mathbf{0}_{2 F(S-1) \times 3} \\
e_{2 F} \otimes \boldsymbol{D} \boldsymbol{G}_{2}
\end{array}\right]} & \text { receiver coordinates } \\
\boldsymbol{e}_{2} \otimes-\boldsymbol{I}_{2 F(S-1)} & \text { satellite clock and instrumental delays } \\
{\left[\begin{array}{c}
\mathbf{0}_{2 F(S-1) \times(S-1)} \\
{\left[\begin{array}{c}
\boldsymbol{\mu} \\
-\boldsymbol{\mu}
\end{array}\right] \otimes \boldsymbol{I}_{S-1}}
\end{array}\right]} & \text { ionospheric delays } \\
{\left[\begin{array}{c}
\mathbf{0}_{2 F(S-1) \times 1} \\
\boldsymbol{e}_{2 F} \otimes \boldsymbol{D} \boldsymbol{\psi}_{2}
\end{array}\right]} & \text { tropospheric zenith delay } \\
{\left[\begin{array}{c}
\mathbf{0}_{3 F(S-1) \times F(S-1)} \\
\boldsymbol{\Lambda} \otimes \boldsymbol{I}_{S-1}
\end{array}\right]} & \text { integer ambiguities. }
\end{array}
$$

The corresponding estimable parameters for between satellite differenced observations are given in Table 2.3. A different yet equivalent formulation of the model is found if a different pivot satellite is chosen when defining the measurement vector (2.57), i.e., the differencing operator $\boldsymbol{D}$. A change of the pivot satellite is a full rank transformation of the measurement vector that does not affect its information content. From a theoretical point of view, the choice of the pivot satellite is thus arbitrary. In order to adjust the model after changing the pivot satellite, one can either apply this full rank transformation to the design matrix $[\boldsymbol{A}, \boldsymbol{B}]$ and leave the parameter vectors unchanged, or adjust the parameter vectors such that the new pivot satellite is used.

Table 2.3: Parameters of full rank between satellite difference model; the design matrices are given in (2.64).

\begin{tabular}{ll}
\hline Parameter & Contains \\
\hline $\boldsymbol{y}$ & $2 F(S-1)$ code observations $\Delta p_{r, f}^{s 1}$ \\
& $2 F(S-1)$ carrier-phase observations $\Delta \phi_{r, f}^{s 1}$ \\
$\boldsymbol{a}$ & $F(S-1)$ double difference ambiguities $N_{21, f}^{s 1}$ \\
$\boldsymbol{b}$ & 3 incremental receiver coordinates $\Delta \boldsymbol{x}_{2} / \Delta \boldsymbol{x}_{21}$ \\
& $2 F(S-1)$ satellite clock offsets $c \tilde{d} t_{, f}{ }_{, f}$ and $c \tilde{\delta} t_{, f}^{s 1}$ \\
& $S-1$ double difference ionospheric delays $I_{21}^{s 1}$ \\
& 1 relative tropospheric zenith delay $T_{\mathrm{z}, 21}$ \\
\hline
\end{tabular}




\subsubsection{Double Difference Model}

Finally, the linearized double difference observation equations follow from (2.14) and (2.15) as

$$
\begin{aligned}
& \Delta p_{r q, f}^{s k}=\boldsymbol{u}_{r}^{s, \mathrm{~T}} \Delta \boldsymbol{x}_{r}-\boldsymbol{u}_{r}^{s, \mathrm{~T}} \Delta \boldsymbol{x}^{s}-\boldsymbol{u}_{q}^{s, \mathrm{~T}} \Delta \boldsymbol{x}_{q}+\boldsymbol{u}_{q}^{s, \mathrm{~T}} \Delta \boldsymbol{x}^{s} \\
&-\boldsymbol{u}_{r}^{k, \mathrm{~T}} \Delta \boldsymbol{x}_{r}+\boldsymbol{u}_{r}^{k, \mathrm{~T}} \Delta \boldsymbol{x}^{k}+\boldsymbol{u}_{q}^{k, \mathrm{~T}} \Delta \boldsymbol{x}_{q}-\boldsymbol{u}_{q}^{k, \mathrm{~T}} \Delta \boldsymbol{x}^{k} \\
&+I_{r q, f}^{s k}+T_{r q}^{s k}+\varepsilon_{r q, f}^{s k} \\
& \Delta \phi_{r q, f}^{s k}(t)=\boldsymbol{u}_{r}^{s, \mathrm{~T}} \Delta \boldsymbol{x}_{r}-\boldsymbol{u}_{r}^{s, \mathrm{~T}} \Delta \boldsymbol{x}^{s}-\boldsymbol{u}_{q}^{s, \mathrm{~T}} \Delta \boldsymbol{x}_{q}+\boldsymbol{u}_{q}^{s, \mathrm{~T}} \Delta \boldsymbol{x}^{s} \quad-\boldsymbol{u}_{r}^{k, \mathrm{~T}} \Delta \boldsymbol{x}_{r}+\boldsymbol{u}_{r}^{k, \mathrm{~T}} \Delta \boldsymbol{x}^{k}+\boldsymbol{u}_{q}^{k, \mathrm{~T}} \Delta \boldsymbol{x}_{q}-\boldsymbol{u}_{q}^{k, \mathrm{~T}} \Delta \boldsymbol{x}^{k} \\
&-I_{r q, f}^{s k}+T_{r q}^{s k}+\lambda_{f} N_{r q, f}^{s k}+\varepsilon_{r q, f}^{s k} .
\end{aligned}
$$

With $S$ the number of satellites that are tracked by both receivers on $F$ frequencies, the measurement vector $\boldsymbol{y}$ is defined as

$$
\boldsymbol{y}=\left[\boldsymbol{p}_{21}^{1, \mathrm{~T}}, \boldsymbol{\phi}_{21}^{1, \mathrm{~T}}\right]^{\mathrm{T}}
$$

with the $F(S-1)$ vectors

$$
\begin{gathered}
\boldsymbol{p}_{21}^{1}=\left[\Delta p_{21,1}^{21}, \ldots, \Delta p_{21,1}^{S 1}, \ldots, \Delta p_{21, F}^{21}, \ldots, \Delta p_{21, F}^{S 1}\right]^{\mathrm{T}} \\
\boldsymbol{\phi}_{21}^{1}=\left[\Delta \phi_{21,1}^{21}, \ldots, \Delta \phi_{21,1}^{S 1}, \ldots, \Delta \phi_{21, F}^{21}, \ldots, \Delta \phi_{21, F}^{S 1}\right]^{\mathrm{T}} .
\end{gathered}
$$

Again, the satellite positions are assumed known and the values of the tropospheric mapping function are assumed identical for both receivers. The double difference measurement vector follows from the measurement vector of the undifferenced model via the consecutive application of the two transformation matrices $\left[-\boldsymbol{I}_{2 F S}, \boldsymbol{I}_{2 F S}\right]$ and $\left[\boldsymbol{I}_{2 F} \otimes \boldsymbol{D}\right]$. Applied to the full rank design matrices of the undifferenced model these transformations zero-fill the columns corresponding to the satellite and receiver clock offsets and directly lead to the full rank double difference model with the partial design matrices

$$
\begin{array}{ll}
\boldsymbol{e}_{2 F} \otimes \boldsymbol{D} \boldsymbol{G}_{2} & \text { receiver coordinates } \\
{\left[\begin{array}{c}
\boldsymbol{\mu} \\
-\boldsymbol{\mu}
\end{array}\right] \otimes \boldsymbol{I}_{S-1}} & \text { ionospheric delays } \\
\boldsymbol{e}_{2 F} \otimes \boldsymbol{D} \boldsymbol{\psi}_{2} & \text { tropospheric zenith delay } \\
{\left[\begin{array}{c}
\mathbf{0}_{F(S-1) \times F(S-1)} \\
\boldsymbol{\Lambda} \otimes \boldsymbol{I}_{S-1}
\end{array}\right]} & \text { integer ambiguities. }
\end{array}
$$

The estimable parameters of the full rank double difference model are listed in Table 2.4.

\subsubsection{Combined Multi-GNSS model}

So far it was assumed that all code and carrier-phase measurements correspond to satellites from the same GNSS. Improved positioning capabilities can be expected when combining the measurements from multiple GNSS as listed in Section 1.1, since the number of satellites that are simultaneously visible is then considerably larger. The fundamental problem that has to be faced in order to formulate a combined multi-GNSS model is outlined and solution approaches are presented.

First the case is considered that the systems that are combined transmit their signals at different carrier-frequencies, which is for example true for GPS and BDS. It is then necessary to extend the 
Table 2.4: Parameters of full rank double difference model; the design matrices are given in (2.69).

\begin{tabular}{ll}
\hline Parameter & Contains \\
\hline $\boldsymbol{y}$ & $F(S-1)$ code observations $\Delta p_{21, f}^{s 1}$ \\
& $F(S-1)$ carrier-phase observations $\Delta \phi_{21, f}^{s 1}$ \\
$\boldsymbol{a}$ & $F(S-1)$ double difference ambiguities $N_{21, f}^{s 1}$ \\
$\boldsymbol{b}$ & 3 incremental receiver coordinates $\Delta \boldsymbol{x}_{2} / \Delta \boldsymbol{x}_{21}$ \\
& $S-1$ double difference ionospheric delays $I_{21}^{s 1}$ \\
& 1 relative tropospheric zenith delay $T_{\mathrm{z}, 21}$ \\
\hline
\end{tabular}

parameter vectors of all frequency dependent terms in the positioning models. For the undifferenced model in Section 2.2.1 this means that we have to introduce separate receiver clocks for the additional frequencies of further systems. This implies that we cannot define double difference ambiguity parameters that correspond to two satellites from different systems, which would anyway not be possible since they do not share a common wavelength. Equivalently, if between satellite differences are computed, it has to be chosen a separate pivot satellite for each system. Essentially, the system model has to be set up separately for each system, with the only common parameters being the receiver coordinates and the relative tropospheric zenith delay.

The situation is quite different if two or more systems are combined that share the same carrierfrequencies. Theoretically it should then be possible to use the same frequency dependent receiver clock offsets for all systems. As it is shown in Hegarty et al. (2005) and Montenbruck et al. (2011) for GPS and Galileo, this assumption is not generally valid due to the potential presence of socalled inter system biases caused by different hardware delays for the signals of different systems. One option to solve this problem is to again use system specific receiver clock parameters, i.e., to use the same strategy that has to be applied when no common frequencies exist. Since the inter system biases are constant hardware specific delays, they can be a priori estimated and subtracted from the measurements (Odijk and Teunissen 2013a,b). This enables setting up the system models as if all measurements were from the same satellite constellation. It is now possible to form double difference ambiguities with satellites from all systems using only a single pivot satellite, or, equivalently, to cancel the receiver clock offsets by computing between satellite differences with satellites from different systems. The calibration of the inter system biases increases the redundancy of the multi-GNSS models, which further improves the positioning capabilities (Odolinski et al. 2014a, 2015a).

\subsection{Stochastic Models}

The following properties are assumed for the additive noise components $\varepsilon_{r, f}^{s}(t)$ and $\epsilon_{r, f}^{s}(t)$ in the observation equations (2.1) and (2.2) for the undifferenced code and carrier-phase measurements.

- The noise components $\varepsilon_{r, f}^{s}(t)$ and $\epsilon_{r, f}^{s}(t)$ follow a zero mean Gaussian distribution.

- No correlations exist between measurements from different receivers.

- No correlations exist between any of the undifferenced measurements made by a single receiver at the same epoch. Depending on the receiver internal processing, this assumption might not be valid for certain combinations of signals from the same satellite to receiver link such as the wideband Galileo E5 signal with E5a or E5b, which are part of the E5 signal itself. 
- No correlations exist between measurements from different epochs. This assumption is shown to be valid in Borre and Tiberius (2000) given that the measurement rate is $1 \mathrm{~Hz}$ or less.

It is further assumed that the variances of the noise components can be parameterized as

$$
\mathrm{E}\left[\left(\varepsilon_{r, f}^{s}(t)\right)^{2}\right]=\left(w_{r}^{s}(t)\right)^{2} \sigma_{p_{f}}^{2} \quad \text { and } \quad \mathrm{E}\left[\left(\epsilon_{r, f}^{s}(t)\right)^{2}\right]=\left(w_{r}^{s}(t)\right)^{2} \sigma_{\phi_{f}}^{2},
$$

where $\mathrm{E}[\cdot]$ denotes the expectation operator. The $2 F$ variances $\sigma_{p_{f}}^{2}$ and $\sigma_{\phi_{f}}^{2}$ are constant and depend on the quality of the hardware and the properties of the signal. Within this dissertation they are assumed identical for all receivers. Typically, the code standard deviations $\sigma_{p_{f}}$ are in the order of a few decimeters and the carrier-phase standard deviations $\sigma_{\phi_{f}}$ are at millimeter level. In case of a multi-GNSS system distinct values are used for each system. The time varying weighting factor $w_{r}^{s}(t)$ is introduced to model the effect of the receiver-satellite elevation angle at time $t$ on the precision of the measurements. It was found that due to the lower signal strength and diffuse multipath the standard deviation of the measurements is higher for satellites at lower elevation angles. We use the exponential weighting function from Euler and Goad (1991):

$$
w_{r}^{s}(t)=1+a_{0} \exp \left(-\frac{E_{r}^{s}(t)}{E_{0}}\right)
$$

where $E_{r}^{s}(t)$ is the elevation angle of satellite $s$ observed at receiver $r$ and $a_{0}$ and $E_{0}$ are constant values, often taken as $a_{0}=10$ and $E_{0}=10^{\circ}$.

The additive noise vector $\boldsymbol{\eta}$ in (2.16) follows the zero mean multivariate Gaussian distribution $\boldsymbol{\eta} \sim \mathcal{G}(\mathbf{0}, \boldsymbol{Q})$, which is fully characterized by the covariance matrix $\boldsymbol{Q}$. Based on the above assumptions, $Q$ is now derived for the functional positioning models from Section 2.2. Let the diagonal weighting matrix $\boldsymbol{W}_{r} \in \mathbb{R}^{S \times S}$ be defined as (the time stamps are omitted)

$$
\boldsymbol{W}_{r}=\operatorname{diag}\left(\left(w_{r}^{1}\right)^{2}, \ldots,\left(w_{r}^{S}\right)^{2}\right) .
$$

The same weighting factors are assumed for both receivers. We therefore define $\boldsymbol{W}=\boldsymbol{W}_{1}=\boldsymbol{W}_{2}$. Further, we define the code and carrier-phase specific $F \times F$ matrices

$$
\boldsymbol{Q}_{\boldsymbol{p}}=\operatorname{diag}\left(\sigma_{p_{1}}^{2}, \ldots, \sigma_{p_{F}}^{2}\right) \quad \text { and } \quad \boldsymbol{Q}_{\boldsymbol{\phi}}=\operatorname{diag}\left(\sigma_{\phi_{1}}^{2}, \ldots, \sigma_{\phi_{F}}^{2}\right)
$$

The covariance matrices $Q$ for the undifferenced functional model of Section 2.2.1, the single difference models from Section 2.2.2, and the double difference model from Section 2.2.3 can now be expressed as

$$
\begin{aligned}
& \boldsymbol{I}_{2} \otimes \operatorname{blkdiag}\left(\boldsymbol{Q}_{\boldsymbol{p}}, \boldsymbol{Q}_{\boldsymbol{\phi}}\right) \otimes \boldsymbol{W} \\
& 2 \cdot \operatorname{blkdiag}\left(\boldsymbol{Q}_{\boldsymbol{p}}, \boldsymbol{Q}_{\boldsymbol{\phi}}\right) \otimes \boldsymbol{W} \\
& \boldsymbol{I}_{2} \otimes \operatorname{blkdiag}\left(\boldsymbol{Q}_{\boldsymbol{p}}, \boldsymbol{Q}_{\boldsymbol{\phi}}\right) \otimes \boldsymbol{D} \boldsymbol{W} \boldsymbol{D}^{\mathrm{T}} \\
& 2 \cdot \operatorname{blkdiag}\left(\boldsymbol{Q}_{\boldsymbol{p}}, \boldsymbol{Q}_{\boldsymbol{\phi}}\right) \otimes \boldsymbol{D} \boldsymbol{W} \boldsymbol{D}^{\mathrm{T}}
\end{aligned}
$$

undifferenced

between receiver differenced

between satellite differenced

double differenced.

If the measurements from multiple epochs are combined by stacking the single epoch measurement vectors, the joint covariance matrix follows from combining the respective single epoch covariance matrices in a blockdiagonal manner. 


\subsection{Integer/Mixed Integer and Real Valued Parameter Estimation}

In the previous sections linearized systems of observation equations used for GNSS positioning have been derived. These systems include both real valued parameters, most prominently the coordinates of the user receiver, and integer parameters in form of the carrier-phase double difference ambiguities. Due to the presence of the integer parameters, it is not sufficient to simply apply standard methods from adjustment theory in linear models, such as (weighted) linear least-squares, if the integer property is to be exploited. In the following, a strategy for solving such a system is presented. The strategy is formulated in a general way so that its use is not restricted to GNSS applications but to any application that includes integer parameters.

We study a system of linear or linearized observation equations in the form

$$
\boldsymbol{y}=\boldsymbol{A a}+\boldsymbol{\eta}
$$

with $\boldsymbol{a} \in \mathbb{Z}^{n}$ the vector of unknown integer parameters, which are linked to the vector of observations $\boldsymbol{y} \in \mathbb{R}^{q}$ via the full-rank matrix $\boldsymbol{A} \in \mathbb{R}^{q \times n}$. The noise vector $\boldsymbol{\eta} \in \mathbb{R}^{q}$ is assumed to follow a zero mean Gaussian distribution with covariance matrix $\boldsymbol{Q}$. The estimation of $\boldsymbol{a}$ is usually decomposed into two steps (Teunissen 1993). In the first step the integer property of $\boldsymbol{a}$ is simply disregarded and the so-called float solution $\hat{\boldsymbol{a}}$ is computed via a standard linear weighted least-squares estimation

$$
\hat{\boldsymbol{a}}=\left(\boldsymbol{A}^{\mathrm{T}} \boldsymbol{Q}^{-1} \boldsymbol{A}\right)^{-1} \boldsymbol{A}^{\mathrm{T}} \boldsymbol{Q}^{-1} \boldsymbol{y} .
$$

This float solution $\hat{\boldsymbol{a}}$ is a minimal sufficient statistic, i.e., it contains the same information about the unknown $\boldsymbol{a}$ as the observation $\boldsymbol{y}$ itself, see A2. The second step, which takes into account the integer property of $\boldsymbol{a}$, can therefore be based on $\hat{\boldsymbol{a}}$ instead of $\boldsymbol{y}$. From the law of error propagation we know that $\hat{\boldsymbol{a}}$ follows a Gaussian distribution:

$$
\hat{\boldsymbol{a}} \sim \mathcal{G}\left(\boldsymbol{a}, \boldsymbol{Q}_{\hat{\boldsymbol{a}}}\right), \quad \text { with } \quad \boldsymbol{Q}_{\hat{\boldsymbol{a}}}=\left(\boldsymbol{A}^{\mathrm{T}} \boldsymbol{Q}^{-1} \boldsymbol{A}\right)^{-1} .
$$

Generally, only some of the unknown parameters may be integer valued. This is the case in the above discussed GNSS positioning applications. The observation model is then of the form (cf. (2.16))

$$
\boldsymbol{y}=\boldsymbol{A} \boldsymbol{a}+\boldsymbol{B} \boldsymbol{b}+\boldsymbol{\eta}
$$

with $\boldsymbol{b} \in \mathbb{R}^{p}$ the vector of real valued unknowns and $\boldsymbol{B} \in \mathbb{R}^{q \times p}$. The combined matrix $[\boldsymbol{A}, \boldsymbol{B}]$ has to be of full column rank. The estimation of $\boldsymbol{a}$ and $\boldsymbol{b}$ can now be decomposed into three steps, where the first one is again the float solution $\hat{\boldsymbol{a}}$ and $\hat{\boldsymbol{b}}$ from a linear weighted least-squares estimation

$$
\left[\begin{array}{c}
\hat{\boldsymbol{a}} \\
\hat{\boldsymbol{b}}
\end{array}\right]=\left(\left[\begin{array}{l}
\boldsymbol{A}^{\mathrm{T}} \\
\boldsymbol{B}^{\mathrm{T}}
\end{array}\right] \boldsymbol{Q}^{-1}\left[\begin{array}{ll}
\boldsymbol{A} & \boldsymbol{B}
\end{array}\right]\right)^{-1}\left[\begin{array}{l}
\boldsymbol{A}^{\mathrm{T}} \\
\boldsymbol{B}^{\mathrm{T}}
\end{array}\right] \boldsymbol{Q}^{-1} \boldsymbol{y} .
$$

Using a blockwise inversion technique like the Banachiewicz formula, see for instance Zhang (2006), which is sometimes also referred to as the matrix inversion lemma, the float estimates $\hat{\boldsymbol{a}}$ and $\hat{b}$ can be explicitly written as

$$
\begin{aligned}
& \hat{\boldsymbol{a}}=\left(\overline{\boldsymbol{A}}^{\mathrm{T}} \boldsymbol{Q}^{-1} \overline{\boldsymbol{A}}\right)^{-1} \overline{\boldsymbol{A}}^{\mathrm{T}} \boldsymbol{Q}^{-1} \boldsymbol{y} \\
& \hat{\boldsymbol{b}}=\left(\overline{\boldsymbol{B}}^{\mathrm{T}} \boldsymbol{Q}^{-1} \overline{\boldsymbol{B}}\right)^{-1} \overline{\boldsymbol{B}}^{\mathrm{T}} \boldsymbol{Q}^{-1} \boldsymbol{y}
\end{aligned}
$$


with $\overline{\boldsymbol{A}}=\boldsymbol{P}_{\boldsymbol{B}}^{\perp} \boldsymbol{A}, \overline{\boldsymbol{B}}=\boldsymbol{P}_{\boldsymbol{A}}^{\perp} \boldsymbol{B}$, and the two projectors $\boldsymbol{P}_{\boldsymbol{A}}^{\perp}=\boldsymbol{I}_{q}-\boldsymbol{A}\left(\boldsymbol{A}^{\mathrm{T}} \boldsymbol{Q}^{-1} \boldsymbol{A}\right)^{-1} \boldsymbol{A}^{\mathrm{T}} \boldsymbol{Q}^{-1}$ and $\boldsymbol{P}_{\boldsymbol{B}}^{\perp}=\boldsymbol{I}_{q}-\boldsymbol{B}\left(\boldsymbol{B}^{\mathrm{T}} \boldsymbol{Q}^{-1} \boldsymbol{B}\right)^{-1} \boldsymbol{B}^{\mathrm{T}} \boldsymbol{Q}^{-1}$, which project onto the orthogonal complement of the range space of $\boldsymbol{A}$ and $\boldsymbol{B}$, respectively. The float solution is Gaussian distributed as

$$
\left[\begin{array}{c}
\hat{a} \\
\hat{b}
\end{array}\right] \sim \mathcal{G}\left(\left[\begin{array}{l}
a \\
b
\end{array}\right],\left[\begin{array}{cc}
\boldsymbol{Q}_{\hat{a}} & \boldsymbol{Q}_{\hat{a} \hat{b}} \\
\boldsymbol{Q}_{\hat{b} \hat{a}} & \boldsymbol{Q}_{\hat{b}}
\end{array}\right]\right)
$$

and delivers a minimal sufficient statistic. It is also the best linear unbiased estimator (BLUE) of $\boldsymbol{a}$ and $\boldsymbol{b}$. In the second step the float solution $\hat{\boldsymbol{a}}$ is used to resolve some or all of the elements of $\boldsymbol{a}$ as integer values. In GNSS applications, the motivation to do so is to improve the accuracy of the estimate of $\boldsymbol{b}$ compared to $\hat{\boldsymbol{b}}$. Although $\hat{\boldsymbol{b}}$ is already the BLUE of the parameter vector $\boldsymbol{b}$, further improvements of the quality of the estimate of $\boldsymbol{b}$ are feasible since such an integer mapping is a non-linear function, i.e., a wider class of estimators is used than the linear estimators. The correlation between $\hat{\boldsymbol{a}}$ and $\hat{\boldsymbol{b}}$ is utilized in the third and final step to correct $\hat{\boldsymbol{b}}$ for the resolved integers. In GNSS positioning with short observation time spans, ambiguity resolution can improve the position accuracy by roughly two orders of magnitude compared to the float solution, depending on the chosen subset of ambiguities and given that the estimated integers are correct (Teunissen 1997a).

The topic of the following chapters is how to find an integer estimate for a certain subset of integer parameters and how to determine this subset based on $\boldsymbol{Q}_{\hat{a}}$ and the realization of $\hat{a}$, which applies to both discussed observation models. The integer fixing takes place either in the original $n$ dimensional parameter space of $\boldsymbol{a}$ or after the reparameterization

$$
\hat{\boldsymbol{a}}^{\prime}=\boldsymbol{Z} \hat{\boldsymbol{a}}, \quad \boldsymbol{Q}_{\hat{\boldsymbol{a}}^{\prime}}=\boldsymbol{Z} \boldsymbol{Q}_{\hat{\boldsymbol{a}}} \boldsymbol{Z}^{\mathrm{T}},
$$

where $\boldsymbol{Z} \in \mathbb{Z}^{n \times n}$ and $|\operatorname{det} \boldsymbol{Z}|=1$, such that $\boldsymbol{Z}^{-1}$ is also an integer unimodular matrix. In the context of GNSS, these transformations are also referred to as admissible ambiguity transformations (Teunissen 1995a). If the full set of integer parameters is resolved, such a transformation may or may not change the resulting integer estimate, depending on the employed estimator, see Chapter 3. The transformation $Z$ is usually chosen such that the probability of a correct integer estimate or the efficiency of the estimator is increased. Since there is a one-to-one relation between the $n$ dimensional integer points for any valid integer transformation, the problem of estimating the full set of integer parameters is equivalent for any choice of $Z$. This is not the case if a subset of the integer parameters is resolved. The problem of partial integer fixing is fundamentally changed by applying an integer transformation, irrespective of the integer mapping that is used, since different choices of $\boldsymbol{Z}$ imply different combinations of the integer parameters that are resolved, which can not be transformed between one another. Possible choices of $\boldsymbol{Z}$ for partial ambiguity resolution in the context of GNSS applications are discussed in Chapter 4. Let the set of indexes that correspond to the integer parameters that are resolved as integers and kept as float values be given by $\mathcal{I}$ and $\overline{\mathcal{I}}$, respectively, where $\mathcal{I} \cup \overline{\mathcal{I}}=\{1, \ldots, n\}$ and $\mathcal{I} \cap \overline{\mathcal{I}}=\emptyset$. All parameters with an index in $\mathcal{I}$ are fixed to integers via the mapping $\mathcal{S}(\cdot)$ :

$$
\check{\boldsymbol{a}}^{\prime}=\mathcal{S}\left(\hat{\boldsymbol{a}}^{\prime}\right), \quad \mathcal{S}(\cdot): \mathbb{R}^{n} \mapsto \mathbb{Z}^{|\mathcal{I}|} .
$$

Note that generally only a subset of the integer parameters is resolved, i.e., the integer solution $\check{\boldsymbol{a}}^{\prime}$ is of dimension $|\mathcal{I}|$ instead of $n$, where $|\mathcal{I}|$ denotes the cardinality of the set $\mathcal{I}$. If system model (2.78) is employed, the float solution $\hat{b}$ is then corrected for the fixed integers

$$
\check{\boldsymbol{b}}=\hat{\boldsymbol{b}}-\boldsymbol{Q}_{\hat{\boldsymbol{b}} \hat{\boldsymbol{a}}_{\mathcal{I}}^{\prime}} \boldsymbol{Q}_{\hat{\boldsymbol{a}}_{\mathcal{I}}^{\prime}}^{-1}\left(\hat{\boldsymbol{a}}_{\mathcal{I}}^{\prime}-\check{\boldsymbol{a}}^{\prime}\right)
$$


with $\boldsymbol{Q}_{\hat{b} \hat{a}_{\mathcal{I}}^{\prime}}=\boldsymbol{Q}_{\hat{b} \hat{a}} \boldsymbol{Z}_{\mathcal{I}}^{\mathrm{T}}$ and $\boldsymbol{Q}_{\hat{\boldsymbol{a}}_{\mathcal{I}}^{\prime}}=\boldsymbol{Z}_{\mathcal{I}} \boldsymbol{Q}_{\hat{a}} \boldsymbol{Z}_{\mathcal{I}}^{\mathrm{T}}$. The quality of the (partially) integer resolved solution $\check{b}$ depends on the properties of the integer mapping $\mathcal{S}(\cdot)$ and is discussed in more detail in Chapters 3-5. Applying the law of error propagation to (2.84) gives

$$
\mathrm{E}[\check{\boldsymbol{b}}]=\boldsymbol{b}-\mathrm{E}\left[\boldsymbol{Q}_{\hat{\boldsymbol{b}} \hat{\boldsymbol{a}}_{\mathcal{I}}^{\prime}} \boldsymbol{Q}_{\hat{\boldsymbol{a}}_{\mathcal{I}}^{\prime}}^{-1}\left(\hat{\boldsymbol{a}}_{\mathcal{I}}^{\prime}-\check{\boldsymbol{a}}^{\prime}\right)\right]
$$

This shows that $\check{\boldsymbol{b}}$ is unbiased if $\mathcal{S}(\cdot)$ is formulated such that $\mathrm{E}\left[\boldsymbol{Q}_{\hat{\boldsymbol{b}} \hat{\boldsymbol{a}}_{\mathcal{I}}^{\prime}} \boldsymbol{Q}_{\hat{\boldsymbol{a}}_{\mathcal{I}}^{\prime}}^{-1}\left(\hat{\boldsymbol{a}}_{\mathcal{I}}^{\prime}-\check{\boldsymbol{a}}^{\prime}\right)\right]=\mathbf{0}$.

The two fundamental questions that have to be answered are how to choose the integer subset $\mathcal{I}$ and how to find an integer estimate for this subset. One can think of a vast amount of ways to approach these problems. Conventionally, the subset $\mathcal{I}$ is restricted to assume only the two values $\mathcal{I}=\{1, \ldots, n\}$ or $\mathcal{I}=\emptyset$ in GNSS applications. That is, either the full set of carrier-phase ambiguities is resolved or none of them is. This problem has been studied extensively and the resulting methods are widely used in the GNSS community. A brief review of the corresponding theory is given in Chapter 3.

The main topic of this dissertation is to investigate the above problems if $\mathcal{I}$ can now assume any of the $2^{n}$ possible realizations, which follow from either including each of the $n$ integer parameters or not. In Chapter 4 we discuss model-driven estimators for which the value of $\mathcal{I}$ is chosen based only on the system model. A more general approach is taken in Chapter 5, where the realization of the subset $\mathcal{I}$ depends also on the measurements and is thus a random variable.

\subsection{Atmospheric Delay Parameters}

The parameter vectors $\boldsymbol{b}$ of the undifferenced, single difference, and double difference functional positioning models formulated in Section 2.2 contain the $S-1$ double difference ionospheric delays $I_{21}^{s 1}$ and one differential tropospheric zenith delay $T_{\mathrm{z}, 21}$. Exploiting the spatial correlation of atmospheric delays in the same geographical region or including external corrections can enable stronger positioning models. This is of very high importance, since this has a strong influence on the positioning capabilities, to the point that it can make the difference of whether or not instantaneous ambiguity resolved positioning is possible. Three ways for treating the atmospheric delays and recommendations for when to use which option are presented in the following.

\subsubsection{Atmosphere Float Model}

The models that were introduced in Sections 2.2 and 2.3 are so-called atmosphere float models. That is, the ionospheric and tropospheric delay parameters are included as unknowns in the system model and estimated along with the user coordinates, the carrier-phase ambiguities, and-if presentthe clock parameters. This strategy does not make use of any prior knowledge and is thus the weakest option. It makes sense to be used for the ionospheric delays if no external corrections are available and if the distance between the receivers exceeds several tens of kilometers, so that the correlations between the absolute ionospheric delays experienced by the signals arriving at the two receivers is too small to be utilized. For the tropospheric delay, this model is usually employed if the residual relative tropospheric zenith delay $T_{\mathrm{z}, 21}$ cannot be assumed negligible. This is the case for large inter receiver distances or if a substantial height difference is present between the receivers. Note that the ionosphere float model can only be used if measurements on at least two frequencies are available, see Table 2.5. 


\subsubsection{Atmosphere Fixed Model}

In the atmosphere fixed model, the atmospheric parameters are completely removed from the system model, thereby increasing the redundancy of the system model by $S-1$ for the ionospheric delays and/or by 1 for the tropospheric delay, cf. Table 2.5. This is the strongest possible atmospheric model. With the ionosphere fixed model, single frequency positioning becomes possible. There are two cases, in which the atmosphere fixed model can be used:

1) Exact a priori information for the atmospheric delays is available. In this case, the code and carrier-phase measurements can simply be corrected for the atmospheric errors, so that the double difference ionospheric delays and/or the relative tropospheric zenith delay vanish and can thus be removed from the system model.

2) The distance between the receivers is sufficiently small so that the atmospheric delays experienced by both receivers are almost identical. In that case, the double difference ionospheric delays and/or the relative tropospheric zenith delay can be assumed negligible and removed from the system model. This assumption is valid for between receiver distances of up to a few kilometers, where for the tropospheric delay additionally the height difference has to be small.

\subsubsection{Atmosphere Weighted Model}

The atmosphere weighted model bridges the gap between the two above described extremes. It is usually only applied to the ionospheric delay parameters, but could also be used for tropospheric parameters (Kleijer 2004). The principle of the ionosphere weighted model (Schaffrin and Bock 1988; Teunissen 1998c; Odijk 2000, 2002) is to treat the (residual) double difference ionospheric delay parameters as stochastic quantities. There are two different cases in which this model can be applied:

1) A priori knowledge about the atmospheric delays is available, but cannot be assumed exact, so that the uncertainty has to be included in the system model. This is for example the case when atmospheric delays are computed at a network of reference stations and then interpolated to the approximate location(s) of the receiver(s).

2) The distance between the receivers is relatively small so that the atmospheric delays experienced by both receivers are correlated, but cannot be assumed identical. The double difference ionospheric delays and/or the relative tropospheric zenith delay can then not be assumed negligible, but can be modeled as a zero mean random variable with distance (and elevation) dependent uncertainty. The ionosphere weighted model is the preferable option for inter receiver distances of up to several tens of kilometers.

The ionosphere weighted model is now formulated. Let the a priori knowledge of the ionospheric delays be given by means of the vector of ionospheric pseudo observations $i$, which is assumed to be Gaussian distributed around the true ionospheric delays $\mathrm{E}[\boldsymbol{i}]$ with covariance matrix $Q_{i}$. The vector $i$ can directly represent the double difference ionospheric delays that are part of the parameter vector $\boldsymbol{b}$, i.e., $\mathrm{E}[\boldsymbol{i}]=\boldsymbol{\iota}$, but can also refer to undifferenced or single difference ionospheric delays that can be transformed to double difference form. Let the matrix $\boldsymbol{B}_{\boldsymbol{i}}$ be defined such that $\boldsymbol{B}_{\boldsymbol{i}} \mathrm{E}[\boldsymbol{i}]=\boldsymbol{B}_{\iota} \iota$, where $\boldsymbol{B}_{\iota}$ contains the columns of the design matrix $\boldsymbol{B}$ of system model (2.16) that correspond to the vector $\iota$ of double difference ionospheric delays. That is, $\boldsymbol{B}_{\boldsymbol{i}}$ transforms the ionospheric pseudo observations to double difference form and properly applies them to 
the observations. The ionospheric pseudo observations can be included in the system model via

$$
\left[\begin{array}{c}
\boldsymbol{y} \\
\boldsymbol{i}
\end{array}\right]=\left[\begin{array}{ccc}
\boldsymbol{A} & \boldsymbol{B}_{\backslash \iota} & \boldsymbol{B}_{\boldsymbol{i}} \\
\mathbf{0} & \mathbf{0} & \boldsymbol{I}
\end{array}\right]\left[\begin{array}{c}
\boldsymbol{a} \\
\boldsymbol{b}_{\backslash \iota} \\
\mathrm{E}[\boldsymbol{i}]
\end{array}\right]+\left[\begin{array}{c}
\boldsymbol{\eta} \\
\boldsymbol{\eta}_{\boldsymbol{i}}
\end{array}\right], \quad \text { with } \quad\left[\begin{array}{c}
\boldsymbol{\eta} \\
\boldsymbol{\eta}_{\boldsymbol{i}}
\end{array}\right] \sim \mathcal{G}\left(\left[\begin{array}{c}
\mathbf{0} \\
\mathbf{0}
\end{array}\right],\left[\begin{array}{cc}
\boldsymbol{Q} & \mathbf{0} \\
\mathbf{0} & \boldsymbol{Q}_{\boldsymbol{i}}
\end{array}\right]\right) .
$$

The parameter vector $\boldsymbol{b}_{\backslash \iota}$ and the design matrix $\boldsymbol{B}_{\backslash_{\iota}}$ follow from $\boldsymbol{b}$ and $\boldsymbol{B}$ by removing the $S-1$ ionospheric delay parameters and the corresponding columns of the design matrix. An equivalent formulation can be found by applying the square and full rank transformation matrix

$$
\left[\begin{array}{cc}
\boldsymbol{I} & -\boldsymbol{B}_{\boldsymbol{i}} \\
\mathbf{0} & \boldsymbol{I}
\end{array}\right]
$$

to the combined observation vector. This results in the system model

$$
\begin{aligned}
{\left[\begin{array}{c}
\boldsymbol{y}-\boldsymbol{B}_{i} \boldsymbol{i} \\
\boldsymbol{i}
\end{array}\right] } & =\left[\begin{array}{ccc}
\boldsymbol{A} & \boldsymbol{B}_{\backslash \iota} & \mathbf{0} \\
\mathbf{0} & \mathbf{0} & \boldsymbol{I}
\end{array}\right]\left[\begin{array}{c}
\boldsymbol{a} \\
\boldsymbol{b}_{\backslash \iota} \\
\mathrm{E}[\boldsymbol{i}]
\end{array}\right]+\left[\begin{array}{c}
\boldsymbol{\eta}^{\prime} \\
\boldsymbol{\eta}_{\boldsymbol{i}}
\end{array}\right], \\
\text { with } \quad\left[\begin{array}{c}
\boldsymbol{\eta}^{\prime} \\
\boldsymbol{\eta}_{\boldsymbol{i}}
\end{array}\right] & \sim \mathcal{G}\left(\left[\begin{array}{l}
\mathbf{0} \\
\mathbf{0}
\end{array}\right],\left[\begin{array}{cc}
\boldsymbol{Q}+\boldsymbol{B}_{\boldsymbol{i}} \boldsymbol{Q}_{\boldsymbol{i}} \boldsymbol{B}_{\boldsymbol{i}}^{\mathrm{T}} & -\boldsymbol{B}_{\boldsymbol{i}} \boldsymbol{Q}_{\boldsymbol{i}} \\
-\boldsymbol{Q}_{\boldsymbol{i}} \boldsymbol{B}_{\boldsymbol{i}}^{\mathrm{T}} & \boldsymbol{Q}_{\boldsymbol{i}}
\end{array}\right]\right) .
\end{aligned}
$$

We recognize $\boldsymbol{i}$ as free variates, see Section A3, which can simply be removed from the model when computing the weighted least-squares estimates, given that we are not interested in estimates for $\mathrm{E}[\boldsymbol{i}]$. The final alternative formulation of the ionosphere weighted model is therefore given by

$$
\boldsymbol{y}-\boldsymbol{B}_{i} \boldsymbol{i}=\left[\begin{array}{ll}
\boldsymbol{A} & \boldsymbol{B}_{\backslash \iota}
\end{array}\right]\left[\begin{array}{c}
\boldsymbol{a} \\
\boldsymbol{b}_{\backslash \iota}
\end{array}\right]+\boldsymbol{\eta}^{\prime}, \quad \text { with } \quad \boldsymbol{\eta}^{\prime} \sim \mathcal{G}\left(\mathbf{0}, \boldsymbol{Q}+\boldsymbol{B}_{i} \boldsymbol{Q}_{\boldsymbol{i}} \boldsymbol{B}_{\boldsymbol{i}}^{\mathrm{T}}\right) .
$$

This formulation is very intuitive: The ionospheric corrections are subtracted from the measurements, and the uncertainty of these corrections is added to the measurement noise. The redundancy of the ionosphere weighted model is the same as the one of the ionosphere fixed model, but the noise contribution is increased, cf. Table 2.5 .

In case 2) from above, the pseudo observations $\boldsymbol{i}$ are simply taken as zero. According to Odijk (2000), the between receiver differential ionospheric delays can be modeled as zero mean Gaussian random variables with the distance dependent standard deviation

$$
\sigma_{I_{r q}^{s}}=w_{r}^{s} \cdot \sqrt{2} \cdot 0.4 \frac{\mathrm{mm}}{\mathrm{km}}
$$

where $w_{r}^{s}$ is an elevation dependent weighting factor, cf. (2.71). Assuming that the between receiver differenced ionospheric delays are uncorrelated for all satellites, the covariance matrix $\boldsymbol{Q}_{\boldsymbol{i}}$ is given by $\boldsymbol{Q}_{\boldsymbol{i}}=\operatorname{diag}\left(\sigma_{I_{r q}^{1}}^{2}, \ldots, \sigma_{I_{r q}^{S}}^{2}\right)$, and $\boldsymbol{B}_{\boldsymbol{i}}=\boldsymbol{B}_{\iota} \boldsymbol{D}$.

The atmosphere weighted model is the most flexible approach to deal with the atmospheric parameters. If the a priori uncertainties as defined by $Q_{i}$ are set to zero or infinity, the atmosphere weighted model reduces to the atmosphere fixed and float model, respectively.

\subsection{Equivalence of Positioning Models}

In the previous section we have seen that the redundancy of the undifferenced, single difference, and double difference positioning models from Section 2.2 is identical. In this section we go one 
Table 2.5: Redundancy, solvability conditions, and components of the additive noise for the ionospheric models. The numbers in the parenthesis correspond to the troposphere fixed model, otherwise the troposphere float model is used.

\begin{tabular}{llll}
\hline & Redundancy $(q-n-p)$ & Solvability condition & Add. noise \\
\hline Ionosphere float & $F(S-1)-S-3(2)$ & $F \geq 2, S \geq 5(4)$ & meas. \\
Ionosphere fixed & $F(S-1)-4(3)$ & $F \geq 1, S \geq 5(4)$ & meas. \\
Ionosphere weighted & $F(S-1)-4(3)$ & $F \geq 1, S \geq 5(4)$ & meas. + iono. \\
\hline
\end{tabular}

step further and investigate whether or not an even stronger relation between these models can be found based on this result. It was shown in Section 2.2 that the single and double difference measurements follow from the undifferenced measurements through simple linear transformations. The corresponding transformation matrices are obviously not of full row rank, since the dimension of the observation vectors of the differenced models is smaller than the dimension of the observation vector of the undifferenced model. Let a full rank square transformation matrix be defined such that the first rows correspond to the transformations that produce the single or double difference measurements from the undifferenced measurements. The remaining rows can be chosen arbitrarily as long as the combined matrix is of full rank and thus invertible. We can now equivalently use the transformed measurement vector instead of the undifferenced measurement vector when computing the weighted least-squares solution. We consider the model

$$
\left[\begin{array}{c}
\boldsymbol{y}_{\mathrm{d}} \\
\boldsymbol{y}^{\mathrm{R}}
\end{array}\right]=\left[\begin{array}{lll}
\boldsymbol{A}_{\mathrm{d}} & \boldsymbol{B}_{\mathrm{d}} & \mathbf{0} \\
\boldsymbol{A}^{\mathrm{R}} & \boldsymbol{B}^{\mathrm{R}} & \boldsymbol{C}
\end{array}\right]\left[\begin{array}{c}
\boldsymbol{a} \\
\boldsymbol{b}_{\mathrm{d}} \\
\boldsymbol{b}^{\mathrm{R}}
\end{array}\right]+\left[\begin{array}{l}
\boldsymbol{\eta}_{\mathrm{d}} \\
\boldsymbol{\eta}^{\mathrm{R}}
\end{array}\right],
$$

where the full rank single or double differenced models as formulated in Sections 2.2 and 2.3 are described by the measurement vector $\boldsymbol{y}_{\mathrm{d}}$, the parameter vectors $\boldsymbol{a}$ and $\boldsymbol{b}_{\mathrm{d}}$ with the corresponding design matrices $\boldsymbol{A}_{\mathrm{d}}$ and $\boldsymbol{B}_{\mathrm{d}}$, and the noise vector $\boldsymbol{\eta}_{\mathrm{d}}$. Note that the vector of integer parameters $\boldsymbol{a}$ is identical for all models, whereas the vector of real valued parameters $\boldsymbol{b}_{\mathrm{d}}$ of the differenced models is different from the one of the undifferenced model, since it is of smaller dimension and can also contain different estimable parameters. It is important to note that the coordinates of the user receiver and-if present-the atmospheric delay parameters of the undifferenced model are also contained in $\boldsymbol{b}_{\mathrm{d}}$, cf. Section 2.2. The second row in (2.91) corresponds to the second part of the transformation matrix. Further, $\boldsymbol{b}^{\mathrm{R}}$ is such that $\left[\boldsymbol{b}_{\mathrm{d}}^{\mathrm{T}}, \boldsymbol{b}^{\mathrm{R}, \mathrm{T}}\right]^{\mathrm{T}}$ is linked to the original parameter vector $\boldsymbol{b}$ of the undifferenced model via an invertible matrix. For the between receiver difference model and the double difference model, $\boldsymbol{b}$ can for instance simply be partitioned into $\boldsymbol{b}_{\mathrm{d}}$ and $\boldsymbol{b}^{\mathrm{R}}$. The combinations of the undifferenced measurements that are chosen in $\boldsymbol{y}^{\mathrm{R}}$ determine the design matrices $\boldsymbol{A}^{\mathrm{R}}, \boldsymbol{B}^{\mathrm{R}}$, and $\boldsymbol{C}$, as well as the properties of $\boldsymbol{\eta}^{\mathrm{R}}$. We can now equivalently use (2.91) instead of the original undifferenced model when computing the weighted least squares solution.

Since the redundancy of the single and double difference models is exactly the same as for the undifferenced model, the dimension of $\boldsymbol{b}^{\mathrm{R}}$ is identical to the dimension of $\boldsymbol{y}^{\mathrm{R}}$, i.e., the reduction in the number of measurements is identical to the reduction in the number of parameters. With the invertible transformations that were applied to obtain (2.91) from the full rank undifferenced model it follows that matrix $C$ is a full rank square matrix. We can thus apply the invertible parameter 
transformation defined through

$$
\left[\begin{array}{ccc}
\boldsymbol{I} & \mathbf{0} & \mathbf{0} \\
\mathbf{0} & \boldsymbol{I} & \mathbf{0} \\
\boldsymbol{A}^{\mathrm{R}} & \boldsymbol{B}^{\mathrm{R}} & \boldsymbol{C}
\end{array}\right]
$$

which leads to the equivalent system model

$$
\left[\begin{array}{c}
\boldsymbol{y}_{\mathrm{d}} \\
\boldsymbol{y}^{\mathrm{R}}
\end{array}\right]=\left[\begin{array}{ccc}
\boldsymbol{A}_{\mathrm{d}} & \boldsymbol{B}_{\mathrm{d}} & \mathbf{0} \\
\mathbf{0} & \mathbf{0} & \boldsymbol{I}
\end{array}\right]\left[\begin{array}{c}
\boldsymbol{a} \\
\boldsymbol{b}_{\mathrm{d}} \\
\boldsymbol{A}^{\mathrm{R}} \boldsymbol{a}+\boldsymbol{B}^{\mathrm{R}} \boldsymbol{b}_{\mathrm{d}}+\boldsymbol{C b}^{\mathrm{R}}
\end{array}\right]+\left[\begin{array}{c}
\boldsymbol{\eta}_{\mathrm{d}} \\
\boldsymbol{\eta}^{\mathrm{R}}
\end{array}\right]
$$

We recognize $\boldsymbol{y}^{\mathrm{R}}$ as free variates, cf. Section A3, which can simply be removed from the system when computing the weighted least-squares estimates of the parameters $\boldsymbol{a}$ and $\boldsymbol{b}_{\mathrm{d}}$. As the weighted least-squares or float solution is the first step when solving the system model, cf. Section 2.4, this means that as long as we are only interested in estimating or modeling the user coordinates, the integer ambiguities, and the atmospheric parameters, but not the clock parameters, the single and double difference models

$$
\boldsymbol{y}_{\mathrm{d}}=\left[\begin{array}{ll}
\boldsymbol{A}_{\mathrm{d}} & \boldsymbol{B}_{\mathrm{d}}
\end{array}\right]\left[\begin{array}{c}
\boldsymbol{a} \\
\boldsymbol{b}_{\mathrm{d}}
\end{array}\right]+\boldsymbol{\eta}_{\mathrm{d}} .
$$

produce the exact same results as the undifferenced model.

In de Jonge (1998) and Odijk (2002) it is discussed why it might still make sense to prefer one model over another. For instance, the double difference model is widely used since it is automatically of full rank, easy to set up, and involves the smallest number of parameters. On the other hand, it is less flexible and does not allow to include measurements from satellites that are not visible at both receivers.

\subsection{Two Step Estimation, PPP-RTK}

The system models in Section 2.2 and 2.3 require that the simultaneous measurements from all receivers are available at a central processing unit, which computes the positioning solution. Typically, a mobile user receiver is provided with the measurements from a nearby reference station, so that the double difference integer ambiguities are estimable and it can determine its precise coordinates. This, however, requires a permanent data link between the two receivers to exchange the measurement data. The basic concept for single receiver precise positioning with estimable carrier-phase integer ambiguities (PPP-RTK) is introduced in the following.

Let the vector of measurements be partitioned in the part $\boldsymbol{y}_{\mathrm{r}}$ containing the observations of the reference station(s) and the part $\boldsymbol{y}_{\mathrm{u}}$ containing the observations of the user receiver. Further, let the combined full rank system of observations be partitioned as

$$
\left[\begin{array}{l}
\boldsymbol{y}_{\mathrm{r}} \\
\boldsymbol{y}_{\mathrm{u}}
\end{array}\right]=\left[\begin{array}{cc}
{\left[\boldsymbol{A}_{\mathrm{r}}, \boldsymbol{B}_{\mathrm{r}}\right]} & \mathbf{0} \\
\boldsymbol{C} & {\left[\boldsymbol{A}_{\mathrm{u}}, \boldsymbol{B}_{\mathrm{u}}\right]}
\end{array}\right]\left[\begin{array}{c}
\boldsymbol{a}_{\mathrm{r}} \\
\boldsymbol{b}_{\mathrm{r}} \\
\boldsymbol{a}_{\mathrm{u}} \\
\boldsymbol{b}_{\mathrm{u}}
\end{array}\right]+\left[\begin{array}{l}
\boldsymbol{\eta}_{\mathrm{r}} \\
\boldsymbol{\eta}_{\mathrm{u}}
\end{array}\right]
$$

where $\left[\boldsymbol{A}_{\mathrm{r}}, \boldsymbol{B}_{\mathrm{r}}\right]$ is a full rank matrix defining the system of measurement equations for the reference stations with the corresponding integer and real valued parameters $\boldsymbol{a}_{\mathrm{r}}$ and $\boldsymbol{b}_{\mathrm{r}}$. Note that no integer parameters are present if only a single reference station is used, since the carrier-phase integer ambiguities can then not be separated from the satellite initial phases and instrumental delays. The 
measurements $\boldsymbol{y}_{\mathrm{u}}$ of the user receiver depend both on the 'network parameters' $\boldsymbol{a}_{\mathrm{r}}$ and $\boldsymbol{b}_{\mathrm{r}}$ via the design matrix $\boldsymbol{C}$ and on the 'user parameters' $\boldsymbol{a}_{\mathrm{u}}$ and $\boldsymbol{b}_{\mathrm{u}}$ through the corresponding design matrices $\boldsymbol{A}_{\mathrm{u}}$ and $\boldsymbol{B}_{\mathrm{u}}$. Without including the network parameters it would not be possible to form the double difference-and thus integer valued-ambiguity vector $\boldsymbol{a}_{\mathrm{u}}$. The combined matrix $\left[\boldsymbol{C}, \boldsymbol{A}_{\mathrm{u}}, \boldsymbol{B}_{\mathrm{u}}\right]$ is therefore not of full rank, but $\left[\boldsymbol{A}_{\mathrm{u}}, \boldsymbol{B}_{\mathrm{u}}\right]$ has to be in order to obtain an overall system matrix that is of full rank. According to the assumptions made in Section 2.3, there are no correlations between measurements from different receivers, i.e., the covariance matrix of the combined noise vector $\left[\boldsymbol{\eta}_{\mathrm{r}}^{\mathrm{T}}, \boldsymbol{\eta}_{\mathrm{u}}^{\mathrm{T}}\right]^{\mathrm{T}}$ is blockdiagonal with $\boldsymbol{Q}_{\boldsymbol{y}_{\mathrm{r}}}$ and $\boldsymbol{Q}_{\boldsymbol{y}_{\mathrm{u}}}$ as its entries.

In order to enable integer ambiguity resolution at the user receiver without using the measurement data from the reference stations, the user has to be provided with estimates of the network parameters $\boldsymbol{a}_{\mathrm{r}}$ and $\boldsymbol{b}_{\mathrm{r}}$ (usually only $\boldsymbol{b}_{\mathrm{r}}$ or parts thereof are required), which can be computed using only the reference stations. We can thus split the solution of (2.95) into two steps:

1) The estimates $\hat{\boldsymbol{a}}_{\mathrm{r}}$ and $\hat{\boldsymbol{b}}_{\mathrm{r}}$ of the network parameters $\boldsymbol{a}_{\mathrm{r}}$ and $\boldsymbol{b}_{\mathrm{r}}$ are determined from

$$
\boldsymbol{y}_{\mathrm{r}}=\left[\boldsymbol{A}_{\mathrm{r}}, \boldsymbol{B}_{\mathrm{r}}\right]\left[\begin{array}{l}
\boldsymbol{a}_{\mathrm{r}} \\
\boldsymbol{b}_{\mathrm{r}}
\end{array}\right]+\boldsymbol{\eta}_{\mathrm{r}} .
$$

They are assumed unbiased and characterized by the covariance matrix $\boldsymbol{Q}_{\mathrm{r}}$. The estimation may involve ambiguity resolution methods, which can lead to an improved precision.

2) The estimates $\hat{\boldsymbol{a}}_{\mathrm{r}}$ and $\hat{\boldsymbol{b}}_{\mathrm{r}}$ are applied as corrections to the user measurements. The user receiver can then determine its positioning solution from the observation model

$$
\boldsymbol{y}_{\mathrm{u}}-\boldsymbol{C}\left[\begin{array}{c}
\hat{\boldsymbol{a}}_{\mathrm{r}} \\
\hat{\boldsymbol{b}}_{\mathrm{r}}
\end{array}\right]=\left[\boldsymbol{A}_{\mathrm{u}}, \boldsymbol{B}_{\mathrm{u}}\right]\left[\begin{array}{l}
\boldsymbol{a}_{\mathrm{u}} \\
\boldsymbol{b}_{\mathrm{u}}
\end{array}\right]+\boldsymbol{\eta}_{\mathrm{u}}^{\prime},
$$

where the covariance matrix of the noise vector $\boldsymbol{\eta}_{\mathrm{u}}^{\prime}$ is given by $\boldsymbol{Q}_{\boldsymbol{y}_{\mathrm{u}}}+\boldsymbol{C} \boldsymbol{Q}_{\mathrm{r}} \boldsymbol{C}^{\mathrm{T}}$. Including the uncertainty of the estimates of the network parameters in $\boldsymbol{\eta}_{\mathrm{u}}^{\prime}$ is essential to obtain a realistic stochastic model.

Many strategies and methods for PPP-RTK have been formulated and demonstrated, e.g., Wübbena et al. (2005), Laurichesse and Mercier (2007), Collins (2008), Ge et al. (2008), Mervart et al. (2008), Laurichesse et al. (2009), Bertiger et al. (2010), Teunissen et al. (2010), Wen et al. (2011), Zhang et al. (2011), Geng et al. (2012), Khodabandeh and Teunissen (2014), and Odijk et al. (2014b). An overview and comparison of these methods providing an interpretation of the different estimable corrections and their influence on the user positioning performance is given in Teunissen and Khodabandeh (2015) and Khodabandeh and Teunissen (2015). It is intuitively clear that the corrections must contain information about the satellite phase biases and initial phases to enable an estimation of the double difference integer ambiguities. Further, it is very beneficial although not required that estimates of the ionospheric delay at the user position are contained in the corrections (Odijk et al. 2012), since it might then be possible to use the ionosphere weighted or even fixed model instead of the ionosphere float model. The advantage of PPP-RTK over traditional RTK methods is that the amount of data that has to be transferred to the user can be reduced, especially if multiple reference stations are used, since only a relatively small number of correction parameters is required instead of a large number of measurements. Further reductions might be possible when exploiting the temporal stability of the bias and clock parameters contained in the corrections. 


\subsection{Model Validation, Measurement Integrity}

The mathematical model as formulated in Sections 2.2 and 2.3 is a simplified description of the observations made by real GNSS receivers. The problem of model validation is to verify whether or not the assumed model is sufficiently consistent with the observation data. Only if this is the case, the quality of the parameter estimates can be sufficiently well described with the statistics that follow from the employed estimator. A brief introduction to statistical testing (Baarda 1968; Teunissen 1998a) for GNSS model validation is given in the following. The methods can be applied to the measurements from a single receiver satellite link, from a single receiver and multiple satellites, or from multiple receivers. We only consider model miss-specifications in the form of a bias on the measurements. We can thus write the null and alternative hypotheses $\mathcal{H}_{0}$ and $\mathcal{H}_{\mathrm{a}}$ as

$$
\mathcal{H}_{0}: \boldsymbol{y}=\boldsymbol{A} \boldsymbol{x}+\boldsymbol{\eta} \quad \text { and } \quad \mathcal{H}_{\mathrm{a}}: \boldsymbol{y}=\boldsymbol{A} \boldsymbol{x}+\boldsymbol{C} \boldsymbol{\nabla}+\boldsymbol{\eta},
$$

with the observation vector $\boldsymbol{y} \in \mathbb{R}^{q}$, the parameter vector $\boldsymbol{x} \in \mathbb{R}^{p}$, and the zero mean Gaussian noise vector $\boldsymbol{\eta}$ characterized by the covariance matrix $\boldsymbol{Q}$. The known matrix $\boldsymbol{C} \in \mathbb{R}^{q \times m}$ describes the type and the unknown vector $\nabla \in \mathbb{R}^{m}$ the magnitude of the $m \leq q-p$ dimensional error. The null hypotheses $\mathcal{H}_{0}$ can be tested against the alternative hypothesis $\mathcal{H}_{\mathrm{a}}$ with the test statistic

$$
T_{m}=\frac{1}{m} \hat{\boldsymbol{e}}^{\mathrm{T}} \boldsymbol{Q}^{-1} \boldsymbol{C}\left(\boldsymbol{C}^{\mathrm{T}} \boldsymbol{Q}^{-1} \boldsymbol{Q}_{\hat{\boldsymbol{e}}} \boldsymbol{Q}^{-1} \boldsymbol{C}\right)^{-1} \boldsymbol{C}^{\mathrm{T}} \boldsymbol{Q}^{-1} \hat{\boldsymbol{e}}
$$

where $\hat{\boldsymbol{e}}=\boldsymbol{y}-\boldsymbol{A} \hat{\boldsymbol{x}}$ is the residual of weighted least-squares under $\mathcal{H}_{0}$ with $\boldsymbol{Q}_{\hat{\boldsymbol{e}}}=\boldsymbol{Q}-$ $\boldsymbol{A}\left(\boldsymbol{A}^{\mathrm{T}} \boldsymbol{Q}^{-1} \boldsymbol{A}\right)^{-1} \boldsymbol{A}^{\mathrm{T}}$. The test statistic $T_{m}$ follows a central F-distribution with $m$ and $\infty$ degrees of freedom if the null hypothesis is true and a non-central F-distribution with non-centrality parameter $\lambda=\nabla^{\mathrm{T}} \boldsymbol{C}^{\mathrm{T}} \boldsymbol{Q}^{-1} \boldsymbol{Q}_{\hat{\boldsymbol{e}}} \boldsymbol{Q}^{-1} \boldsymbol{C} \boldsymbol{\nabla}$ if the alternative hypothesis is true:

$$
\mathcal{H}_{0}: T_{m} \sim F(m, \infty, 0) \text { and } \mathcal{H}_{\mathrm{a}}: T_{m} \sim F(m, \infty, \lambda) .
$$

With a chosen level of significance $\alpha_{m}$, which is the probability that $\mathcal{H}_{0}$ is rejected given that it is true, the null hypothesis is rejected in favor of the alternative hypothesis if $T_{m} \geq F_{\alpha_{m}}(m, \infty, 0)$ with $\alpha_{m}=P\left(T_{m}>F_{\alpha_{m}}(m, \infty, 0) \mid \mathcal{H}_{0}\right)$.

In GNSS applications it is not a priori clear which alternative hypothesis $\mathcal{H}_{\mathrm{a}}$ has to be considered. A testing framework for such a situation is given by the iterative detection, identification, and adaptation (DIA) procedure (Teunissen 1990, 1998a). The three steps are described as:

1) Detection: An overall model test is performed to check whether or not the null hypothesis can be assumed valid. The null hypothesis is rejected if $T_{q-p} \geq F_{\alpha_{q-p}}(q-p, \infty, 0)$, where

$$
T_{q-p}=\frac{\hat{\boldsymbol{e}}^{\mathrm{T}} \boldsymbol{Q}^{-1} \hat{\boldsymbol{e}}}{q-p} .
$$

2) Identification: If the null hypothesis is rejected by the overall model test, the model missspecifications that are likely to have caused the rejection have to be identified, i.e., the matrix $C$ is to be specified. To this end, the test statistic $T_{m_{i}}$ is evaluated for different choices $C_{i}$ for the matrix $\boldsymbol{C}$, where the dimension $m_{i}$ of the model error can be different for each choice. The most likely alternative hypothesis is the one for which

$$
\frac{T_{m_{i}}}{F_{\alpha_{m_{i}}}\left(m_{i}, \infty, 0\right)}
$$


is maximum. In GNSS applications common model errors are a carrier-phase cycle slip or an outlier in a code observation, for which $m=1$ and $\boldsymbol{C}$ reduces to a vector $\boldsymbol{c}$. The test statistic (2.99) is then given by $T_{1}=w^{2}$ with

$$
w=\frac{\boldsymbol{c}^{\mathrm{T}} \boldsymbol{Q}^{-1} \hat{\boldsymbol{e}}}{\sqrt{\boldsymbol{c}^{\mathrm{T}} \boldsymbol{Q}^{-1} \boldsymbol{Q}_{\hat{e}} \boldsymbol{Q}^{-1} \boldsymbol{c}}} .
$$

The test statistic $w$ is normally distributed with $w \sim \mathcal{G}(0,1)$ under $\mathcal{H}_{0}$. Screening each observation for the presence of an outlier, i.e, choosing $\boldsymbol{c}_{i}$ as unit vectors with a 1 on the $i$ th position, is known as data snooping (Baarda 1968).

The question is now how to choose the levels of significance $\alpha_{q-p}$ and $\alpha_{m_{i}}$ required to compute the critical value $F_{\alpha_{q-p}}(q-p, \infty, 0)$ of the overall model test (2.101) and the critical values $F_{\alpha_{m_{i}}}\left(m_{i}, \infty, 0\right)$ in (2.102). It is reasonable to construct the overall model test (2.101) such that a specific model error $\boldsymbol{C} \boldsymbol{\nabla}$ is detected with the same probability as it would be by $T_{m_{i}}$ with a critical value of $F_{\alpha_{m_{i}}}\left(m_{i}, \infty, 0\right)$, i.e., such that both tests have the same power $\gamma$. Let $\lambda\left(\alpha_{m_{i}}, \gamma, m_{i}\right)$ denote the non-centrality parameter that results for a test for which the level of significance $\alpha_{m_{i}}$, the power $\gamma$, and the dimension of the error $m_{i}$ is known. The above condition then translates to

$$
\lambda\left(\alpha_{q-p}, \gamma, q-p\right)=\lambda\left(\alpha_{m_{i}}, \gamma, m_{i}\right), \forall i
$$

One can now for instance determine the non-centrality parameter for a one dimensional error with $m_{i}=1$ and user defined values for $\alpha_{1}$ and $\gamma$. The minimum absolute value $|\nabla|$ that can be detected for a specific vector $\boldsymbol{c}$ and the determined non-centrality parameter $\lambda\left(\alpha_{1}, \gamma, 1\right)$ is referred to as the minimum detectable bias. The critical values for the overall model test and identification tests with $m_{i} \neq 1$ can then be determined with the constant non-centrality parameter and power of the test.

3) Adaptation: The model is corrected for the miss-specification that was identified as the most likely one. In GNSS applications this can be accomplished by replacing the null-hypothesis with the identified alternative hypothesis. After this correction it has to be verified that the newly found null-hypothesis is an acceptable choice, which means that the detection step andif required-also the other steps of the DIA procedure are repeated.

A formulation of the DIA procedure suitable to be run in parallel to a recursive least-squares estimator is given in Teunissen (1990) and Teunissen (1998a).

The slightly different navigation integrity concept known as receiver autonomous integrity monitoring (RAIM) has been used since the early days of GPS and was specifically developed for safety critical applications (Brown and Hwang 1986; Lee 1986; Parkinson and Axelrad 1988; Sturza 1988; Walter and Enge 1995). The fundamental idea of RAIM is that the occurrence of positioning errors larger than given alert limits must not exceed a user-defined probability if a solution is provided. In conventional RAIM, only the code observations are used and the redundancy is utilized to ensure the consistency of the single pseudoranges with the positioning solution and to detect and optionally exclude faulty measurements. The DIA procedure as presented above cannot directly serve as a RAIM algorithm, since the probability of large positioning errors is not available (Imparato 2016). 


\section{Full Ambiguity Resolution-a Review}

In this chapter a brief overview of the theory of full ambiguity resolution is given and exemplary methods are introduced. The index set $\mathcal{I}$ of the ambiguities that are resolved can only assume the two values $\mathcal{I}=\{1, \ldots, n\}$ or $\mathcal{I}=\emptyset$, i.e., either the full set of integer parameters is resolved or none of them is. In Section 3.1 the class of integer estimators is discussed, for which the index set is deterministic and given by $\mathcal{I}=\{1, \ldots, n\}$. For the more elaborate but also more flexible integer aperture estimators, which are discussed in Section 3.2, the index set can also depend on the measurement data, i.e., the index set $\mathcal{I}$ is a discrete random variable assuming both possible realizations with a certain probability. For the sake of notational simplicity, $\hat{\boldsymbol{a}}$ and $\check{\boldsymbol{a}}$ are used instead of $\hat{\boldsymbol{a}}^{\prime}$ and $\check{\boldsymbol{a}}^{\prime}$ for the ambiguity estimates after the transformation with $\boldsymbol{Z}$.

\subsection{The Class of Integer Estimators}

The index set $\mathcal{I}$ of an integer estimator is deterministic with $\mathcal{I}=\{1, \ldots, n\}$ and therefore the fixed solution $\check{\boldsymbol{a}}=\mathcal{S}(\hat{\boldsymbol{a}})$ is always an $n$ dimensional integer vector, i.e., $\mathcal{S}(\cdot): \mathbb{R}^{n} \mapsto \mathbb{Z}^{n}$, cf. (2.83). Such an estimator can be fully described by the regions $S_{\boldsymbol{z}} \subset \mathbb{R}^{n}, \forall \boldsymbol{z} \in \mathbb{Z}^{n}$, which denote the point set that is mapped to the same integer $z$ via $\mathcal{S}(\cdot)$

$$
S_{\boldsymbol{z}}=\left\{\boldsymbol{x} \in \mathbb{R}^{n} \mid \boldsymbol{z}=\mathcal{S}(\boldsymbol{x})\right\}, \quad \forall \boldsymbol{z} \in \mathbb{Z}^{n}
$$

With these regions the corresponding integer estimator (2.83) can be explicitly written as

$$
\check{\boldsymbol{a}}=\sum_{\boldsymbol{z} \in \mathbb{Z}^{n}} s_{\boldsymbol{z}}(\hat{\boldsymbol{a}}) \boldsymbol{z}
$$

with the indicator function

$$
s_{\boldsymbol{z}}(\hat{\boldsymbol{a}})= \begin{cases}1 & \text { if } \hat{\boldsymbol{a}} \in S_{\boldsymbol{z}} \\ 0 & \text { else. }\end{cases}
$$

The constraints that have to be imposed on the construction of the so-called pull-in regions $S_{z}$ have been formulated in Teunissen (1999a):

$$
\begin{aligned}
& \text { (i) } \bigcup_{\boldsymbol{z} \in \mathbb{Z}^{n}} S_{\boldsymbol{z}}=\mathbb{R}^{n} \\
& \text { (ii) } \operatorname{int}\left(S_{\boldsymbol{z}}\right) \cap \operatorname{int}\left(S_{\boldsymbol{u}}\right)=\emptyset, \quad \forall \boldsymbol{z}, \boldsymbol{u} \in \mathbb{Z}^{n}, \boldsymbol{z} \neq \boldsymbol{u} \\
& \text { (iii) } S_{\boldsymbol{z}}=S_{\mathbf{0}}+\boldsymbol{z}, \quad \forall \boldsymbol{z} \in \mathbb{Z}^{n} .
\end{aligned}
$$

The first two conditions state that the regions $S_{z}$ have to cover the space $\mathbb{R}^{n}$ without gaps and overlaps, meaning that the integer mapping $\mathcal{S}(\cdot)$ leads to a unique integer estimate for any realization of the float solution $\hat{\boldsymbol{a}} \in \mathbb{R}^{n}$. The third condition states that the regions $S_{z}$ are translated copies of each other. This implies that a shift of the float solution $\hat{\boldsymbol{a}}$ by an arbitrary integer vector $\boldsymbol{z} \in \mathbb{Z}^{n}$ also leads to a shift of the integer estimate $\check{\boldsymbol{a}}$ by $\boldsymbol{z}$, meaning that the integer remove-restore technique can be applied, i.e., one can work with the fractional parts of the float estimates $\hat{\boldsymbol{a}}$ instead of with the complete entries. Three specific realizations of integer estimators are presented later in this section. They are proven to fulfill the properties (3.4) in Teunissen (1999a). 
A similar yet more restrictive concept is given by origin-symmetric extremal convex bodies, whose interior contains exactly one grid point. As shown by Minkowski, integer shifted versions of such a body scaled by a factor of $\frac{1}{2}$ cover $\mathbb{R}^{n}$ without gaps and overlaps (Gruber and Lekkerkerker 1987), i.e., the constraints (3.4) are automatically fulfilled.

\subsubsection{Quality of the Estimates}

The probabilistic properties of the estimate $\check{a}$ of the integer parameters and-if present-the estimate $\check{\boldsymbol{b}}$ of the real valued parameters are fully described by their parameter distribution. Let $p_{\hat{\boldsymbol{a}}}(\boldsymbol{x})$ denote the Gaussian probability density function (pdf) of the float estimate $\hat{a}$. The probability mass function (pmf) of the estimate $\check{\boldsymbol{a}}$ for a given integer estimator as defined by its pull-in regions $S_{z}$ follows as

$$
P(\check{\boldsymbol{a}}=\boldsymbol{z})=P\left(\hat{\boldsymbol{a}} \in S_{\boldsymbol{z}}\right)=\int_{S_{\boldsymbol{z}}} p_{\hat{\boldsymbol{a}}}(\boldsymbol{x}) \mathrm{d} \boldsymbol{x}, \quad \forall \boldsymbol{z} \in \mathbb{Z}^{n} .
$$

An important measure for the quality of the estimate $\check{\boldsymbol{a}}$ is the probability of correct integer estimates $P_{\mathrm{S}}=P(\check{\boldsymbol{a}}=\boldsymbol{a})$, also referred to as the ambiguity success rate in GNSS applications. The probability of incorrect integer estimates or ambiguity failure rate follows as $P_{\mathrm{F}}=1-P_{\mathrm{S}}$.

In GNSS applications, one is much more interested in the distribution of the fixed estimates $\check{b}$ of the real valued parameters as defined in (2.84). According to Teunissen (1999b) and Teunissen (2002) the pdf of $\breve{b}$ is given by

$$
p_{\check{\boldsymbol{b}}}(\boldsymbol{\beta})=\sum_{\boldsymbol{z} \in \mathbb{Z}^{n}} p_{\hat{\boldsymbol{b}} \mid \hat{\boldsymbol{a}}}(\boldsymbol{\beta} \mid \boldsymbol{z}) P(\check{\boldsymbol{a}}=\boldsymbol{z}),
$$

where the conditional pdf $p_{\hat{\boldsymbol{b}} \mid \hat{\boldsymbol{a}}}(\boldsymbol{\beta} \mid \boldsymbol{x})$ is Gaussian with the conditional mean $\boldsymbol{b}-\boldsymbol{Q}_{\hat{\boldsymbol{b}} \hat{\boldsymbol{a}}} \boldsymbol{Q}_{\hat{\boldsymbol{a}}}^{-1}(\boldsymbol{a}-\boldsymbol{x})$ and the conditional covariance matrix $\boldsymbol{Q}_{\hat{b} \mid \hat{a}}=\boldsymbol{Q}_{\hat{b}}-\boldsymbol{Q}_{\hat{b} \hat{a}} \boldsymbol{Q}_{\hat{a}}^{-1} \boldsymbol{Q}_{\hat{a} \hat{b}}$. The pdf of $\check{b}$ is thus an infinite sum of weighted conditional distributions with the values of the pmf of $\check{a}$ as weights. Using the deterministic subset $\mathcal{I}=\{1, \ldots, n\}$ in (2.85) shows that the estimate $\check{\boldsymbol{b}}$ is unbiased if $\mathrm{E}[\check{\boldsymbol{a}}]=\boldsymbol{a}$. This is the case if the region $S_{z}$ is symmetric with respect to $z$. Given that the ambiguity failure rate $P_{\mathrm{F}}$ is sufficiently small, i.e., that $P(\check{\boldsymbol{a}}=\boldsymbol{a}) \approx 1$, the pdf of $\check{\boldsymbol{b}}$ can be approximated by $p_{\hat{\boldsymbol{b}} \mid \hat{\boldsymbol{a}}}(\boldsymbol{\beta} \mid \boldsymbol{a})$, which represents a Gaussian distribution with mean value $\boldsymbol{b}$ and covariance matrix $\boldsymbol{Q}_{\hat{b} \mid \hat{a}}$. The ambiguity success rate is thus also an important measure for the quality of the estimates $\breve{b}$.

In GNSS positioning applications the conditional precision $\boldsymbol{Q}_{\hat{b} \mid \hat{a}}$ is usually much better than the float precision $\boldsymbol{Q}_{\hat{b}}$, but wrong ambiguity estimates are likely to cause large positioning errors that often exceed the ones of the float estimate $\hat{b}$. Reliable positioning therefore requires that the ambiguity failure rate is limited to a user-defined maximum tolerable value. The failure rate of an integer estimator is inherently defined by the underlying system model as represented by $\boldsymbol{Q}_{\hat{a}}$ and the shape of the pull-in regions. We can therefore only evaluate the corresponding success rate and use the result to decide about whether or not to apply the integer estimator. This is the principle of model-driven reliable full ambiguity resolution, since the decision of whether or not to resolve the full set of ambiguities is only based on the description of the system model (Teunissen and Verhagen 2008). 


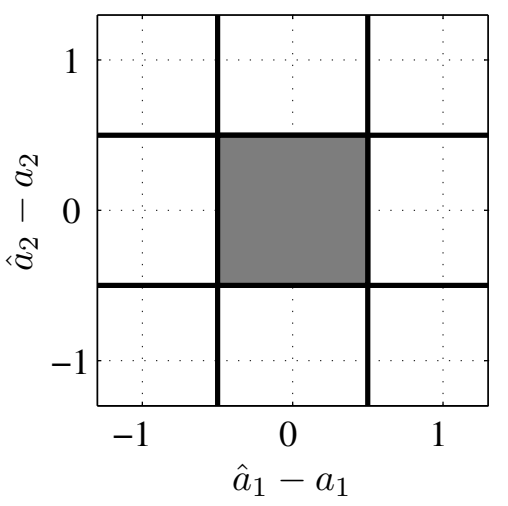

(a) Element-wise rounding

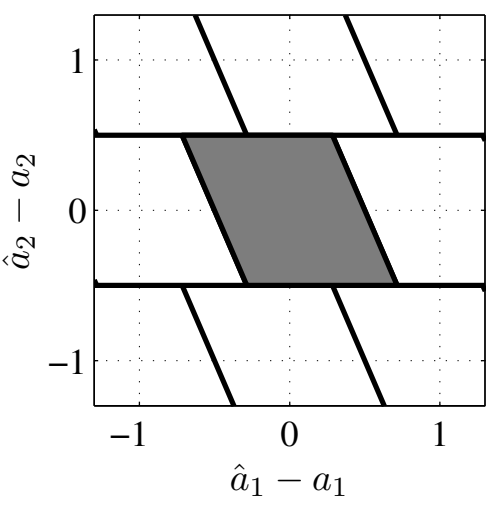

(b) Integer bootstrapping

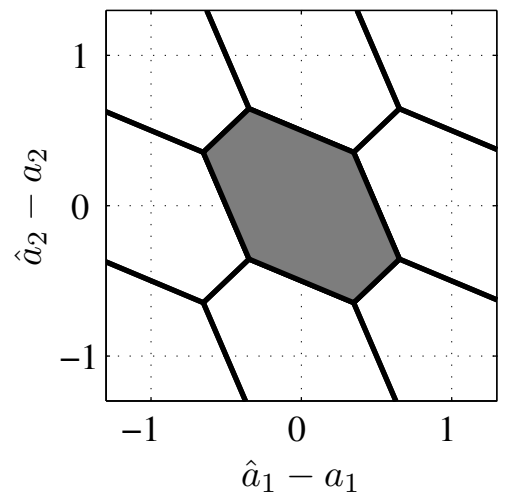

(c) Integer least-squares

Figure 3.1: Pull-in regions $S_{\boldsymbol{z}}$ of integer estimators for a two dimensional example. The regions that lead to correct integer estimates are marked in gray.

\subsubsection{Element-Wise Rounding}

The simplest approach to define the integer mapping $\mathcal{S}(\cdot)$ is to round each of the entries of the input vector to its nearest integer value. The corresponding integer solution $\check{\boldsymbol{a}}_{\mathrm{IR}}$ is given by

$$
\check{\boldsymbol{a}}_{\mathrm{IR}}=\left[\begin{array}{c}
\left\lfloor\hat{a}_{1}\right\rceil \\
\vdots \\
\left\lfloor\hat{a}_{n}\right\rceil
\end{array}\right],
$$

where $L \cdot\rceil$ is used to denote rounding to the nearest integer. Correlations between the elements of the float solution $\hat{\boldsymbol{a}}$ are completely neglected by this estimator. The pull-in regions $S_{z}$ of elementwise rounding are given by

$$
S_{\boldsymbol{z}}=\left\{\boldsymbol{x} \in \mathbb{R}^{n}|| x_{i}-z_{i} \mid \leq \frac{1}{2}, i=1, \ldots, n\right\}, \quad \forall \boldsymbol{z} \in \mathbb{Z}^{n}
$$

They are illustrated in Figure 3.1 for a two dimensional example.

The success rate $P_{\mathrm{S}, \mathrm{IR}}$ of element-wise rounding is difficult to compute exactly, but can be bounded (Teunissen 1998b). It depends on the chosen ambiguity parameterization as defined by $Z$.

\subsubsection{Integer Bootstrapping}

The element-wise rounding method can be modified so as to consider the correlations between the elements of the float solution. The rounding operator is then not applied in parallel to all elements $\hat{a}_{i}$ of $\hat{\boldsymbol{a}}$, but rather in a sequential manner, where the decision for the previous elements is taken into account. We thus obtain a sequential conditional rounding method, also referred to as Babai solution (Babai 1986) in lattice theory or as integer bootstrapping in the context of GNSS (Blewitt 1989; Xu et al. 1995; Teunissen 1998b). Similar approaches are also used in the field of communications, where the elements of $\boldsymbol{a}$ are not necessarily integers but can assume values from a discrete set of symbols. Methods for sequential detection/decoding are referred to as decision feedback equalization or successive interference cancellation. The integer solution clearly depends 
on the order of the sequential fixing. Starting from the last entry and proceeding in reversed order, the elements of the integer bootstrapping solution $\check{\boldsymbol{a}}_{\mathrm{IB}}$ are given by

$$
\begin{aligned}
\check{a}_{\mathrm{IB}, n} & =\left\lfloor\hat{a}_{n}\right\rceil \\
\check{a}_{\mathrm{IB}, n-1} & =\left\lfloor\hat{a}_{n-1 \mid n}\right\rceil=\left\lfloor\hat{a}_{n-1}-\sigma_{\hat{a}_{n-1} \hat{a}_{n}} \sigma_{\hat{a}_{n}}^{-2}\left(\hat{a}_{n}-\check{a}_{\mathrm{IB}, n}\right)\right\rceil \\
\vdots & \\
\check{a}_{\mathrm{IB}, 1} & =\left\lfloor\hat{a}_{1 \mid 2, \ldots, n}\right\rceil=\left\lfloor\hat{a}_{1}-\sum_{i=2}^{n} \sigma_{\hat{a}_{1} \hat{a}_{i \mid I}} \sigma_{\hat{a}_{i \mid I}^{-2}}\left(\hat{a}_{i \mid I}-\check{a}_{\mathrm{IB}, i}\right)\right\rceil,
\end{aligned}
$$

where $\hat{a}_{i \mid I}$ denotes the $i$ th element of the float estimate $\hat{\boldsymbol{a}}$ conditioned on the previously sequentially rounded estimates with the indexes $I=\{i+1, \ldots, n\}$. The coefficients required to compute $\hat{a}_{i \mid I}$ are obtained from the triangular decomposition $\boldsymbol{Q}_{\hat{\boldsymbol{a}}}=\boldsymbol{L}^{\mathrm{T}} \boldsymbol{D} \boldsymbol{L}$ of the float covariance matrix, where $\boldsymbol{L} \in \mathbb{R}^{n \times n}$ is a unit left lower triangular matrix and $\boldsymbol{D} \in \mathbb{R}^{n \times n}$ a diagonal matrix. The non-zero off-diagonal entries of $\boldsymbol{L}$ are given as

$$
L_{i j}=\sigma_{\hat{a}_{j} \hat{a}_{i \mid I}} \sigma_{\hat{a}_{i \mid I}}^{-2}, \quad 1 \leq i<j \leq n,
$$

and the elements on the diagonal of $\boldsymbol{D}$ as $D_{i i}=\sigma_{\hat{a}_{i \mid I}}^{2}, i=1, \ldots, n$. Let the vector of conditional float estimates be defined as $\hat{\boldsymbol{a}}_{\mathrm{c}}=\left[\hat{a}_{1 \mid 2, \ldots, n}, \ldots, \hat{a}_{n}\right]^{\mathrm{T}}$. With $\hat{\boldsymbol{a}}-\check{\boldsymbol{a}}_{\mathrm{IB}}=\boldsymbol{L}^{\mathrm{T}}\left(\hat{\boldsymbol{a}}_{\mathrm{c}}-\check{\boldsymbol{a}}_{\mathrm{IB}}\right)$, the pull-in regions $S_{z}$ of integer bootstrapping follow as

$$
S_{\boldsymbol{z}}=\left\{\boldsymbol{x} \in \mathbb{R}^{n}|| \boldsymbol{c}_{i}^{\mathrm{T}} \boldsymbol{L}^{-\mathrm{T}}(\boldsymbol{x}-\boldsymbol{z}) \mid \leq \frac{1}{2}, i=1, \ldots, n\right\}, \quad \forall \boldsymbol{z} \in \mathbb{Z}^{n},
$$

where $c_{i}$ is a canonical unit vector with 1 as its $i$ th entry. They are illustrated in Figure 3.1 for a two dimensional example.

The success rate $P_{\mathrm{S}, \mathrm{IB}}$ of integer bootstrapping can be computed exactly:

$$
P_{\mathrm{S}, \mathrm{IB}}=\prod_{i=1}^{n}\left(2 \Phi\left(\frac{1}{2 \sigma_{\hat{a}_{i \mid I}}}\right)-1\right) .
$$

The success rate of the integer bootstrapping estimator depends on both the parameterization of the float solution and the order in which it is applied to the parameters.

\subsubsection{Integer Least-Squares}

The integer least-squares estimator accounts for all correlations between the elements of the float solution $\hat{\boldsymbol{a}}$. The integer estimate $\check{\boldsymbol{a}}_{\mathrm{ILS}}$ is the integer vector with the smallest distance to the float solution in the metric of the covariance matrix $\boldsymbol{Q}_{\hat{a}}$

$$
\check{\boldsymbol{a}}_{\mathrm{ILS}}=\underset{\boldsymbol{z} \in \mathbb{Z}^{n}}{\operatorname{argmin}}\|\hat{\boldsymbol{a}}-\boldsymbol{z}\|_{\boldsymbol{Q}_{\hat{a}}}^{2}
$$

with $\|\boldsymbol{x}\|_{\boldsymbol{Q}}^{2}=\boldsymbol{x}^{\mathrm{T}} \boldsymbol{Q}^{-1} \boldsymbol{x}$. The integer least-squares estimate cannot be obtained by simple rounding operations but requires a search for the closest integer grid point, which is a non-trivial task, especially if the dimension $n$ of the above minimization problem is high. This problem is a special case of the closest point problem in lattice theory, which is also a common problem in other fields 
such as communication theory. A comprehensive survey of closest point search methods is given in the tutorial paper Agrell et al. (2002). The pull-in regions $S_{z}$ of integer least-squares are given by

$$
S_{\boldsymbol{z}}=\left\{\boldsymbol{x} \in \mathbb{R}^{n} \mid\|\boldsymbol{x}-\boldsymbol{z}\|_{\boldsymbol{Q}_{\hat{\boldsymbol{a}}}}^{2} \leq\|\boldsymbol{x}-\boldsymbol{u}\|_{\boldsymbol{Q}_{\hat{\boldsymbol{a}}}}^{2}, \forall \boldsymbol{u} \in \mathbb{Z}^{n}\right\}, \quad \forall \boldsymbol{z} \in \mathbb{Z}^{n} .
$$

The regions $S_{z}$ are also referred to as the Voronoi cells of the integer grid points $\boldsymbol{z}$, which are discussed in mathematics textbooks such as Cassels (1971) and applied to the problem of GPS ambiguity resolution in Hassibi and Boyd (1998) and Xu (2006). They are illustrated in Figure 3.1 for a two dimensional example.

The integer least-squares estimator is proven in Teunissen (1999a) to be optimal in the sense of maximizing the probability of correct integer estimates, i.e.,

$$
P\left(\check{\boldsymbol{a}}_{\mathrm{ILS}}=\boldsymbol{a}\right) \geq P(\check{\boldsymbol{a}}=\boldsymbol{a})
$$

with $\check{\boldsymbol{a}}$ from an arbitrary integer estimator. The success rate of integer least-squares does not depend on the parameterization of the float solution, and equivalent estimates $\check{\boldsymbol{a}}_{\mathrm{ILS}}$ are obtained for any admissible transformation $Z$. Since the success rate of integer least-squares is difficult to be evaluated exactly, various bounds and approximations have been derived. An overview over these is given in, e.g., Verhagen (2003) and Verhagen et al. (2013). Within this dissertation the main focus is on reliable ambiguity resolution with a maximum failure rate constraint. We are therefore only interested in upper bounds of the integer least-squares failure rate. From (3.15) we know that the integer bootstrapping failure rate (3.12) is such an upper bound. The analyses in Feng and Wang (2011) and Verhagen et al. (2013) show that it is a tight upper bound in GNSS applications if a prior decorrelation transformation is applied, see Section 3.1.5.

It is noted that if the integer least-squares estimator (3.13) is used in the estimation framework of Section 2.4 for the problem with combined integer and real valued unknowns, then the solution $\check{\boldsymbol{a}}_{\mathrm{ILS}}$ and the corresponding $\check{\boldsymbol{b}}$ is also the solution of (Teunissen 1993)

$$
\left\{\check{\boldsymbol{a}}_{\mathrm{ILS}}, \check{\boldsymbol{b}}\right\}=\underset{\boldsymbol{z} \in \mathbb{Z}^{n}, \boldsymbol{\beta} \in \mathbb{R}^{p}}{\operatorname{argmin}}\|\boldsymbol{y}-\boldsymbol{A} \boldsymbol{z}-\boldsymbol{B} \boldsymbol{\beta}\|_{\boldsymbol{Q}}^{2},
$$

which is the natural definition of the least-squares solution with integer and real valued unknowns.

The integer least-squares estimate $\check{\boldsymbol{a}}_{\mathrm{ILS}}$ can be computed as follows. Let the Cholesky decomposition of the inverse covariance matrix be given by $\boldsymbol{Q}_{\hat{\boldsymbol{a}}}^{-1}=\tilde{\boldsymbol{L}} \tilde{\boldsymbol{L}}^{\mathrm{T}}$, with $\tilde{\boldsymbol{L}} \in \mathbb{R}^{n \times n}$ a left lower triangular matrix. The weighted squared distance between the float estimate $\hat{\boldsymbol{a}}$ and an arbitrary integer $z$ can then be expressed in the recursive form

$$
\begin{aligned}
d(\boldsymbol{z})=\|\hat{\boldsymbol{a}}-\boldsymbol{z}\|_{\boldsymbol{Q}_{\hat{\boldsymbol{a}}}}^{2} & =\sum_{i=1}^{n}\left(\sum_{j=i}^{n} \tilde{L}_{j i}\left(\hat{a}_{j}-z_{j}\right)\right)^{2} \\
& =\sum_{i=1}^{n} e_{i}\left(z_{i}, \ldots, z_{n}\right)
\end{aligned}
$$

where the distance increments $e_{i}(\cdot)$ only depend on the elements $z_{j}$ with $j \geq i$. Starting from level $i=n$, the partial squared distance $d_{i}\left(z_{i}, \ldots, z_{n}\right)$ can be expressed as

$$
d_{i}\left(z_{i}, \ldots, z_{n}\right)=d_{i+1}\left(z_{i+1}, \ldots, z_{n}\right)+e_{i}\left(z_{i}, \ldots, z_{n}\right),
$$


with the initial value $d_{n+1}=0$. The value of $d(\boldsymbol{z})$ is then obtained from the relation $d(\boldsymbol{z})=d_{1}(\boldsymbol{z})$. With all distance increments $e_{i}(\cdot)$ being non-negative, solving problem (3.13) can be interpreted as a tree search problem. Nodes of the search tree correspond to partial squared distances $d_{i}(\cdot)$ and branches, which correspond to different integer values, are given the corresponding weight $e_{i}(\cdot)$. Only nodes of the tree with $d_{i}(\cdot) \leq \lambda^{2}$ are considered, where the search radius $\lambda \in \mathbb{R}_{+}$ has to be properly defined. This means that in the final level of the tree all integer vectors within an hyper-ellipsoid around $\hat{\boldsymbol{a}}$ are found. The integer vector with the smallest squared distance is the integer least-squares solution $\check{\boldsymbol{a}}_{\mathrm{ILS}}$. The size parameter $\lambda$ of the search space can be set using the integer bootstrapping solution $\lambda^{2}=\left\|\hat{\boldsymbol{a}}-\check{\boldsymbol{a}}_{\mathrm{IB}}\right\|_{Q_{\hat{a}}}^{2}$, so as to ensure that at least one integer vector is contained. This strategy of using an ellipsoidal search space is referred to as sphere decoder in communications (Viterbo and Boutros 1999). The idea was originally proposed as the Pohst strategy in lattice theory (Fincke and Pohst 1985). A refinement is given by the Schnorr-Euchner strategy (Schnorr and Euchner 1994). Its main idea is to evaluate the different integer hypotheses in each level in the order of increasing distance increments and to reduce the size of the search space to $\lambda^{2}=d(\boldsymbol{z})$ whenever a candidate $\boldsymbol{z}$ has been found. The first integer candidate used with the Schnorr-Euchner strategy is again the integer bootstrapping solution. The search routine of the least-squares ambiguity decorrelation adjustment (LAMBDA) method for GNSS ambiguity resolution (Teunissen 1995b; de Jonge and Tiberius 1996) is a variant of the Pohst strategy. A numerical comparison of the efficiency of different search strategies for GNSS examples is provided in Jazaeri et al. (2012).

\subsubsection{Decorrelation/Reduction Transformations}

If the covariance matrix $\boldsymbol{Q}_{\hat{a}}$ is diagonal, both element-wise rounding and integer bootstrapping are identical to integer least-squares and thus optimal. This also implies that the integer bootstrapping failure rate, which can be easily computed, is identical to the integer least-squares failure rate. These estimators should therefore be applied in a decorrelated space through a proper choice of the transformation matrix $\boldsymbol{Z}$. Due to the integer constraints of $\boldsymbol{Z}$ it is not possible to completely remove the correlations between the elements of the float solution, but they can be largely reduced. Although the integer least-squares solution is equivalent for each choice of $Z$, the complexity required for its computation is considerably reduced after decorrelation for GNSS ambiguity resolution, since the number of visited nodes of the search tree is much smaller. Also, the ordering of the elements of the transformed float solution should be considered when defining $Z$. It is reasonable to choose the most precise float parameters as the last entries for integer bootstrapping, since these are fixed first and the integer solution of all further components is based on the decision. It is also beneficial for integer least-squares to start with the most precise float estimates, since this may allow to remove large parts of the search tree already in the first levels and thus lead to faster solutions.

The input of the integer estimator $\mathcal{S}(\cdot)$ can be written by means of the pseudo observation model $\hat{\boldsymbol{a}}=\boldsymbol{a}+\boldsymbol{\nu}$ with the noise vector $\boldsymbol{\nu} \sim \mathcal{G}\left(\mathbf{0}, \boldsymbol{Q}_{\hat{\boldsymbol{a}}}\right)$. With the unimodular integer transformation matrix $\boldsymbol{Z}$ (2.82) the transformed system follows as $\boldsymbol{Z} \hat{\boldsymbol{a}}=\boldsymbol{Z} \boldsymbol{a}+\boldsymbol{\nu}^{\prime}$ with $\boldsymbol{\nu}^{\prime} \sim \mathcal{G}\left(\mathbf{0}, \boldsymbol{Z} \boldsymbol{Q}_{\hat{\boldsymbol{a}}} \boldsymbol{Z}^{\mathrm{T}}\right)$. Instead of estimating $\boldsymbol{a}$, the transformed integer vector $\boldsymbol{Z} \boldsymbol{a}$ is estimated. Integer decorrelation aims at designing $\boldsymbol{Z}$ such that the elements of $\boldsymbol{Z} \hat{\boldsymbol{a}}$ are largely uncorrelated. With $\boldsymbol{Q}_{\hat{\boldsymbol{a}}}^{-1}=\tilde{\boldsymbol{L}} \tilde{\boldsymbol{L}}$, i.e., $\boldsymbol{Q}_{\hat{\boldsymbol{a}}}=\tilde{\boldsymbol{L}}^{-\mathrm{T}} \tilde{\boldsymbol{L}}^{-1}$, this means that the rows of $\boldsymbol{Z} \tilde{\boldsymbol{L}}^{-\mathrm{T}}$ should be orthogonal. The above pseudo observation model can equivalently be written as

$$
\tilde{\boldsymbol{L}}^{\mathrm{T}} \hat{\boldsymbol{a}}=\tilde{\boldsymbol{L}}^{\mathrm{T}} \boldsymbol{a}+\tilde{\boldsymbol{\nu}}
$$




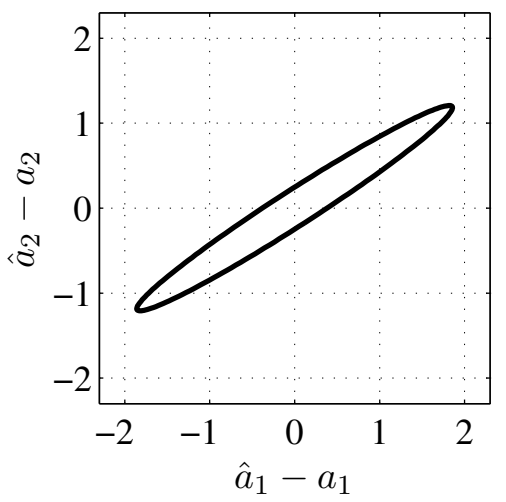

(a) Before decorrelation

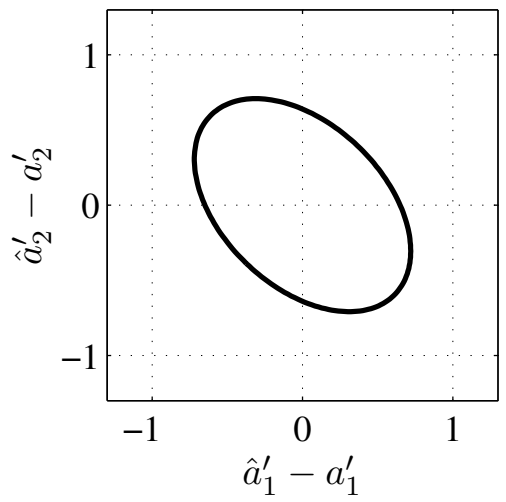

(b) After decorrelation

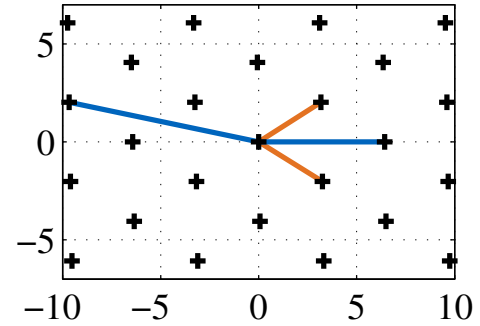

(c) Lattice with basis vectors

Figure 3.2: Confidence ellipses of float estimates $\hat{\boldsymbol{a}}$ before and after decorrelation with $Z$ for a two dimensional example. The black crosses represent the corresponding lattice $\mathcal{L}$ with the basis vectors from $\tilde{\boldsymbol{L}}^{\mathrm{T}}$ in blue and from $\tilde{\boldsymbol{L}}^{\mathrm{T}} \boldsymbol{Z}^{-1}$ in orange.

with $\tilde{\boldsymbol{\nu}} \sim \mathcal{G}\left(\mathbf{0}, \boldsymbol{I}_{n}\right)$. This is the notation that is used in lattice theory, where the lattice $\mathcal{L}$ is given by $\mathcal{L}=\left\{\boldsymbol{x} \in \mathbb{R}^{n} \mid \boldsymbol{x}=\tilde{\boldsymbol{L}}^{\mathrm{T}} \boldsymbol{z}, \boldsymbol{z} \in \mathbb{Z}^{n}\right\}$. The columns of $\tilde{\boldsymbol{L}}^{\mathrm{T}}$ are the basis vectors of the lattice. The exact same lattice is obtained when using $\tilde{\boldsymbol{L}}^{\mathrm{T}} \boldsymbol{Z}^{-1}$ as a basis matrix, with $\boldsymbol{Z}^{-1}$ an arbitrary integer unimodular matrix. We can thus rewrite (3.19) as

$$
\tilde{\boldsymbol{L}}^{\mathrm{T}} \hat{\boldsymbol{a}}=\tilde{\boldsymbol{L}}^{\mathrm{T}} \boldsymbol{Z}^{-1} \boldsymbol{Z} \boldsymbol{a}+\tilde{\boldsymbol{\nu}}
$$

and estimate the transformed ambiguities $\boldsymbol{Z} \boldsymbol{a}$ instead of $\boldsymbol{a}$ using the lattice basis $\tilde{\boldsymbol{L}}^{\mathrm{T}} \boldsymbol{Z}^{-1}$. The concept of an integer transformation via $Z$ is thus equivalent to choosing a different lattice basis. Assuming that $\boldsymbol{Z}$ leads to perfectly decorrelated float estimates, i.e., that the rows of $\boldsymbol{Z} \tilde{\boldsymbol{L}}^{-\mathrm{T}}$ are orthogonal, the columns of $\tilde{\boldsymbol{L}}^{\mathrm{T}} \boldsymbol{Z}^{-1}$ are orthogonal as well. The integer decorrelation problem can thus also be formulated as the problem of finding a preferably orthogonal lattice basis, which is equivalent to finding lattice vectors of shortest length. This problem is therefore referred to as lattice reduction (Wübben et al. 2011). An illustration of the confidence ellipses of the float estimates $\hat{\boldsymbol{a}}$ before and after a decorrelation transformation and the corresponding lattice with the two sets of basis vectors is shown in Figure 3.2 for a two dimensional example.

Examples of decorrelation methods used in the context of GNSS are given in, e.g., Lenstra et al. (1982), Seysen (1993), Teunissen (1995b), Xu (2001), Chang et al. (2005), and Günther and Henkel (2012). A numerical comparison is provided in Jazaeri et al. (2014). Integer decorrelation combined with an efficient search strategy is the basis for fast integer least-squares implementations such as the LAMBDA method (Teunissen 1995b) or a modified version thereof (Chang et al. 2005).

\subsection{The Class of Integer Aperture Estimators}

The index set $\mathcal{I}$ of an integer aperture estimator can also depend on the float estimate $\hat{\boldsymbol{a}}$ and is thus a discrete random variable assuming the two values $\mathcal{I}=\{1, \ldots, n\}$ and $\mathcal{I}=\emptyset$. The point set $\Omega \subseteq \mathbb{R}^{n}$ for which all integer parameters are resolved is called the aperture region (Teunissen 2003b). Accordingly, the index set $\mathcal{I}$ is given by

$$
\mathcal{I}= \begin{cases}\{1, \ldots, n\} & \text { if } \hat{\boldsymbol{a}} \in \Omega \\ \emptyset & \text { else. }\end{cases}
$$


The aperture region $\Omega$ defines the choice of the index set $\mathcal{I}$ but not the integer solution required to define the mapping $\mathcal{S}(\cdot)$ for $\mathcal{I}=\{1, \ldots, n\}$. With the pull-in regions $S_{\boldsymbol{z}}$ (3.1) of a valid integer estimator we define

$$
\Omega_{z}=\Omega \cap S_{z}, \quad \forall z \in \mathbb{Z}^{n} .
$$

Since the pull-in regions $S_{\boldsymbol{z}}, \forall \boldsymbol{z} \in \mathbb{Z}^{n}$, cover the whole $\mathbb{R}^{n}$ without gaps and overlaps, it follows that $\Omega=\bigcup_{z \in \mathbb{Z}^{n}} \Omega_{z}$. The integer estimate $\check{\boldsymbol{a}}$ of an integer aperture estimator can be explicitly written as

$$
\check{\boldsymbol{a}}= \begin{cases}\sum_{\boldsymbol{z} \in \mathbb{Z}^{n}} \omega_{\boldsymbol{z}}(\hat{\boldsymbol{a}}) \boldsymbol{z} & \text { if } \mathcal{I}=\{1, \ldots, n\} \\ {[]} & \text { else, }\end{cases}
$$

with []$\in \mathbb{Z}^{0}$ a zero dimensional vector and the indicator function

$$
\omega_{\boldsymbol{z}}(\hat{\boldsymbol{a}})= \begin{cases}1 & \text { if } \hat{\boldsymbol{a}} \in \Omega_{\boldsymbol{z}} \\ 0 & \text { else }\end{cases}
$$

The following constraint has to be imposed when formulating the aperture region $\Omega$ :

$$
\Omega=\Omega+\boldsymbol{z}, \quad \forall \boldsymbol{z} \in \mathbb{Z}^{n} .
$$

This constraint is the equivalence of condition (iii) in (3.4) for integer estimators. It states that the aperture region is invariant for any integer shift, meaning that a shift of the float solution $\hat{\boldsymbol{a}}$ by an arbitrary integer vector $z \in \mathbb{Z}^{n}$ has no impact on the resulting index set $\mathcal{I}$. This implies that one can again equivalently work with the fractional part of $\hat{\boldsymbol{a}}$ instead of with the complete entries.

The class of integer estimators as introduced in Section 3.1 is contained in the class of integer aperture estimators and follows by choosing the aperture region as $\Omega=\mathbb{R}^{n}$.

\subsubsection{Quality of the Estimates}

With $p_{\hat{\boldsymbol{a}}}(\boldsymbol{x})$ the Gaussian pdf of the float estimate $\hat{\boldsymbol{a}}$, the pmf of $\check{\boldsymbol{a}}$ for a given integer aperture estimator as defined by its aperture pull-in regions $\Omega_{z}$ follows as

$$
\begin{aligned}
& P(\check{\boldsymbol{a}}=\boldsymbol{z})=P\left(\hat{\boldsymbol{a}} \in \Omega_{\boldsymbol{z}}\right)=\int_{\Omega_{\boldsymbol{z}}} p_{\hat{\boldsymbol{a}}}(\boldsymbol{x}) \mathrm{d} \boldsymbol{x}, \quad \forall \boldsymbol{z} \in \mathbb{Z}^{n} \\
& \text { and } P(\check{\boldsymbol{a}}=[])=P\left(\hat{\boldsymbol{a}} \in \mathbb{R}^{n} \backslash \Omega\right)=\int_{\mathbb{R}^{n} \backslash \Omega} p_{\hat{\boldsymbol{a}}}(\boldsymbol{x}) \mathrm{d} \boldsymbol{x}
\end{aligned}
$$

In order to assess the quality of the estimates $\check{\boldsymbol{a}}$ and to evaluate and compare different integer aperture estimators, three different cases with their respective probabilities can be distinguished for an integer aperture estimator:

$$
\begin{array}{lll}
\text { Correct integer estimates: } & \hat{\boldsymbol{a}} \in \Omega_{\boldsymbol{a}}, & \text { success rate } P_{\mathrm{S}}=P(\check{\boldsymbol{a}}=\boldsymbol{a}) \\
\text { Incorrect integer estimates: } & \hat{\boldsymbol{a}} \in \bigcup_{\boldsymbol{z} \in \mathbb{Z}^{n} \backslash \boldsymbol{a}} \Omega_{\boldsymbol{z}}, & \text { failure rate } P_{\mathrm{F}}=\sum_{\boldsymbol{z} \in \mathbb{Z}^{n} \backslash \boldsymbol{a}} P(\check{\boldsymbol{a}}=\boldsymbol{z}) \\
\text { No integer estimates: } & \hat{\boldsymbol{a}} \in \mathbb{R}^{n} \backslash \Omega, & \text { undecided rate } P_{\mathrm{U}}=1-P_{\mathrm{S}}-P_{\mathrm{F}} .
\end{array}
$$

That is, in addition to correct or incorrect integer estimates as for integer estimators there is now a third option which is no integer estimates. 
The distribution of the fixed estimates $\breve{b}$ of the real valued parameters as defined in (2.84) is given by (Verhagen 2005)

$$
p_{\check{\boldsymbol{b}}}(\boldsymbol{\beta})=\sum_{\boldsymbol{z} \in \mathbb{Z}^{n}} p_{\hat{\boldsymbol{b}} \mid \hat{\boldsymbol{a}}}(\boldsymbol{\beta} \mid \boldsymbol{z}) P(\check{\boldsymbol{a}}=\boldsymbol{z})+\int_{\mathbb{R}^{n} \backslash \Omega} p_{\hat{\boldsymbol{b}} \mid \hat{\boldsymbol{a}}}(\boldsymbol{\beta} \mid \boldsymbol{x}) p_{\hat{\boldsymbol{a}}}(\boldsymbol{x}) \mathrm{d} \boldsymbol{x} .
$$

The pdf of $\check{b}$ is the sum of two parts. The first part is an infinite sum of weighted conditional distributions with the probabilities of $\check{a}$ assuming the corresponding $n$ dimensional integer vector as weights, which corresponds to the case that $\mathcal{I}=\{1, \ldots, n\}$. The case $\mathcal{I}=\emptyset$ is covered by the second part, which is the integral of the joint distribution of $\hat{\boldsymbol{a}}$ and $\hat{\boldsymbol{b}}$ over all realizations of $\hat{\boldsymbol{a}}$ that lead to $\mathcal{I}=\emptyset$. From (2.85) follows that the estimates $\breve{\boldsymbol{b}}$ are unbiased if the region $\Omega_{\boldsymbol{z}}$ is symmetric with respect to $\boldsymbol{z}$. If the failure rate $P_{\mathrm{F}}$ is sufficiently small, the pdf of $\breve{b}$ given that $\mathcal{I}=\{1, \ldots, n\}$ can be approximated by $p_{\check{\boldsymbol{b}} \mid \mathcal{I}}(\boldsymbol{\beta} \mid\{1, \ldots, n\}) \approx p_{\hat{\boldsymbol{b}} \mid \hat{\boldsymbol{a}}}(\boldsymbol{\beta} \mid \boldsymbol{a})$, i.e., the precision that can be expected from $\check{b}$ if the determined index set is $\mathcal{I}=\{1, \ldots, n\}$ can be described with the conditional covariance matrix $\boldsymbol{Q}_{\hat{b} \mid \hat{a}}$.

It was discussed in Section 3.1.1 that the ambiguity failure rate $P_{\mathrm{F}}$ has to be limited to a maximum tolerable value for reliable GNSS positioning. The concept of integer aperture estimation has the big advantage over integer estimation that the failure rate can be controlled by means of properly defining the aperture region $\Omega$. That is, irrespective of the strength of the underlying system model, a maximum failure rate can be guaranteed. The probability of resolving the integer ambiguities depends on the choice of the aperture region and is given by $P(\mathcal{I}=\{1, \ldots, n\})=P_{\mathrm{S}}+P_{\mathrm{F}}$. Since the decision of whether or not to resolve the full set of ambiguities now also depends on the measurement data through the realization of the float solution $\hat{\boldsymbol{a}}$, this is referred to as data-driven reliable full ambiguity resolution (Teunissen and Verhagen 2008). When used with a user-defined maximum tolerable failure rate, integer aperture estimators serve as an overall estimation and validation procedure (Verhagen and Teunissen 2006; Teunissen and Verhagen 2009).

\subsubsection{Optimal Integer Aperture Estimation}

The optimal integer aperture estimator is introduced in Teunissen (2005a) as the solution of the optimization problem

$$
\max _{\Omega_{z}} P_{\mathrm{S}} \quad \text { s.t. } \quad P_{\mathrm{F}}=\gamma,
$$

with $\Omega_{z}$ a valid aperture pull-in region and the success and failure rates $P_{\mathrm{S}}$ and $P_{\mathrm{F}}$ as defined in (3.27). In other words, if the integer aperture estimators are formulated such that a fixed failure rate $P_{\mathrm{F}}=\gamma$ is obtained, then the optimal integer aperture estimator leads to the highest possible probability of resolving the integer parameters. The aperture pull-in regions $\Omega_{z}$ of the optimal estimator can be derived from a modified version of the Neyman-Pearson Lemma and are given by

$$
\Omega_{\boldsymbol{z}}=\left\{\boldsymbol{x} \in S_{\boldsymbol{z}} \mid \sum_{\boldsymbol{u} \in \mathbb{Z}^{n} \backslash \boldsymbol{z}} \exp \left(-\frac{1}{2}\|\boldsymbol{x}-\boldsymbol{u}\|_{\boldsymbol{Q}_{\hat{a}}}^{2}\right) \leq \mu \exp \left(-\frac{1}{2}\|\boldsymbol{x}-\boldsymbol{z}\|_{\boldsymbol{Q}_{\hat{a}}}^{2}\right)\right\}, \quad \forall \boldsymbol{z} \in \mathbb{Z}^{n},
$$

where $S_{z}$ denotes the pull-in region of integer least-squares and the critical value $\mu$ is chosen so as to satisfy the fixed failure rate criterion $P_{\mathrm{F}}=\gamma$. The decision of whether or not float the estimate $\hat{\boldsymbol{a}}$ resides within $\Omega$, and therefore of whether or not to resolve the full set of integer parameters, includes the evaluation of an infinite sum. This sum cannot be computed exactly but has to be approximated by neglecting all terms whose contribution is sufficiently small, see Section 4.3.4. 


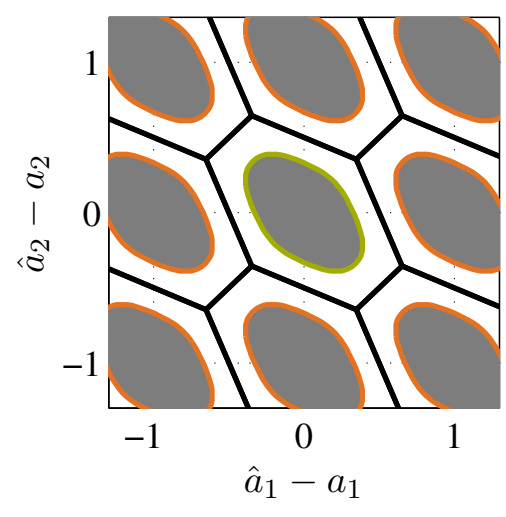

Figure 3.3: The aperture pull-in regions $\Omega_{z}$ of the optimal integer aperture estimator are shown in gray for a two dimensional example, where green and orange edges indicate correct and incorrect integer estimates, respectively. The boundaries of the pull-in regions $S_{\boldsymbol{z}}$ of the underlying integer estimator are drawn in black.

For the computation of the optimal integer aperture estimator, first the integer least-squares solution $\check{\boldsymbol{a}}_{\mathrm{ILS}}$ is determined. By evaluating the condition in (3.30) for the region $\Omega_{\check{\boldsymbol{a}}_{\mathrm{ILS}}}$ it is then checked if the integer least-squares solution can be accepted. The search for the integer vectors used for the approximation is computationally more efficient after applying a decorrelation transformation, but the results are equivalent when using the search space as defined in Section 4.3.4. Methods for determining the model dependent critical value $\mu$ for a fixed failure rate implementation are discussed in Section 5.5. The regions $\Omega_{z}$ of the optimal integer aperture estimator are shown in Figure 3.3 for a two dimensional example.

Various integer aperture estimators have been proposed in the literature, e.g., the ratio test (Euler and Schaffrin 1991), the difference test (Tiberius and de Jonge 1995), the projector test (Han 1997; Wang et al. 1998), the F-ratio test (Frei and Beutler 1990), the ellipsoidal integer aperture estimator (Teunissen 2003a), integer aperture bootstrapping (Teunissen 2005b), integer aperture least-squares (Teunissen 2005c), and the penalized integer aperture estimator (Teunissen 2004). An overview and comparison of these methods is provided in Verhagen (2005). The first three options are briefly introduced in the following. They are based on testing the best integer candidate $\check{\boldsymbol{a}}_{\mathrm{ILS}}$ against the second best integer candidate and are therefore much easier to compute than the optimal integer aperture estimator. They produce identical results for any admissible parameterization via $Z$. They are proven to be valid integer aperture estimators in Verhagen and Teunissen (2006).

\subsubsection{The Difference Test}

The difference test is introduced for GNSS ambiguity validation in Tiberius and de Jonge (1995). It is based on testing the integer least-squares estimate $\check{\boldsymbol{a}}_{\mathrm{ILS}}$ against the best counter hypothesis $\overline{\boldsymbol{a}}$, which is given by

$$
\overline{\boldsymbol{a}}=\underset{\boldsymbol{z} \in \mathbb{Z}^{n} \backslash \check{\boldsymbol{a}}_{\mathrm{ILS}}}{\operatorname{argmin}}\|\hat{\boldsymbol{a}}-\boldsymbol{z}\|_{\boldsymbol{Q}_{\hat{\boldsymbol{a}}}}^{2} .
$$

The integer least-squares solution is accepted if

$$
\|\hat{\boldsymbol{a}}-\overline{\boldsymbol{a}}\|_{\boldsymbol{Q}_{\hat{a}}}^{2}-\left\|\hat{\boldsymbol{a}}-\check{\boldsymbol{a}}_{\mathrm{ILS}}\right\|_{\boldsymbol{Q}_{\hat{a}}}^{2} \geq \mu
$$

with the critical value $\mu$. The values produced by the difference test are always non-negative. This combined estimation and validation strategy is an integer aperture estimator defined by the aperture pull-in regions

$$
\Omega_{\boldsymbol{z}}=\left\{\boldsymbol{x} \in S_{\boldsymbol{z}} \mid\|\boldsymbol{x}-\boldsymbol{u}\|_{\boldsymbol{Q}_{\hat{a}}}^{2}-\|\boldsymbol{x}-\boldsymbol{z}\|_{\boldsymbol{Q}_{\hat{a}}}^{2} \geq \mu, \forall \boldsymbol{u} \in \mathbb{Z}^{n} \backslash \boldsymbol{z}\right\}, \quad \forall \boldsymbol{z} \in \mathbb{Z}^{n}
$$




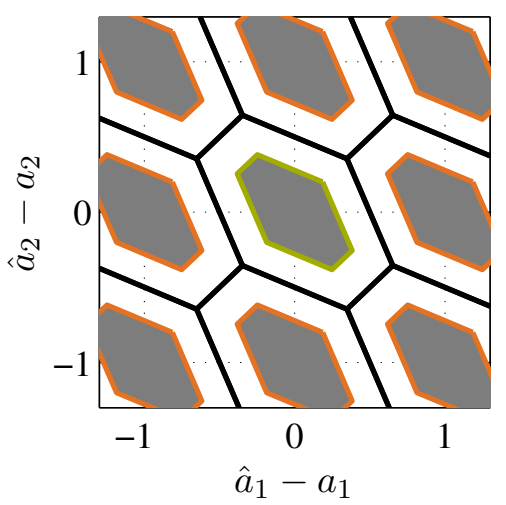

(a) Difference test

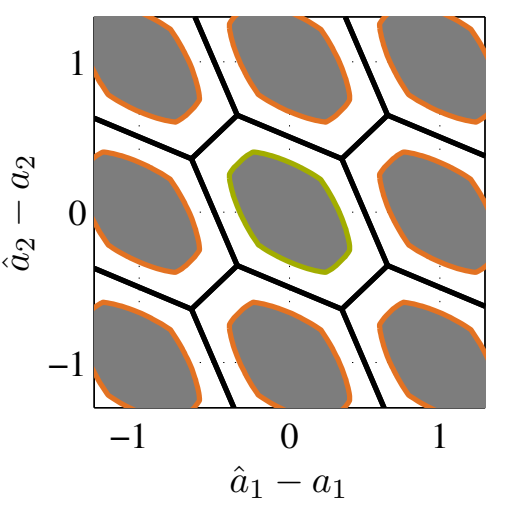

(b) Ratio test

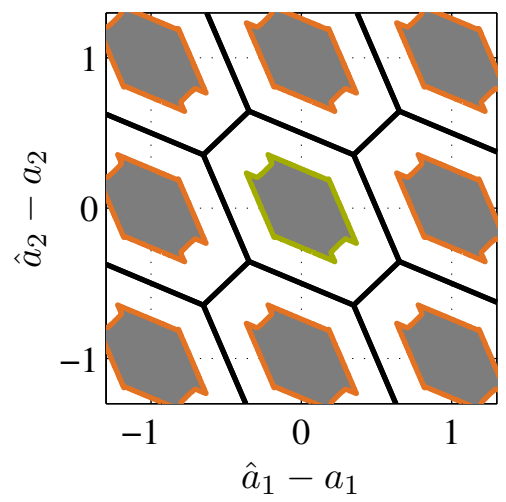

(c) Projector test

Figure 3.4: The aperture pull-in regions $\Omega_{z}$ are shown in gray for a two dimensional example, where green and orange edges indicate correct and incorrect integer estimates, respectively. The boundaries of the pull-in regions $S_{z}$ of the underlying integer estimator are drawn in black.

where $S_{z}$ are the pull-in regions of integer least-squares. It is noted that the regions $\Omega_{z}$ of the difference test follow from the optimal aperture pull-in regions if the sum in (3.30) is replaced by its maximum term. It can therefore be expected that the difference test performs close to optimal if the distribution of the float estimate is highly peaked around $\boldsymbol{a}$, such that the contributions of the further terms of the sum are sufficiently small. By properly choosing the aperture parameter $\mu$, a fixed failure rate $P_{\mathrm{F}}$ can be guaranteed, see Section 5.5. For $\mu=0, \Omega_{z}$ is identical to the respective integer least-squares pull-in region. The regions $\Omega_{z}$ of the difference test based integer aperture estimator are shown in Figure 3.4 for a two dimensional example. They are constructed as the intersection of half-spaces.

\subsubsection{The Ratio Test}

A different test of the same form is the ratio test as introduced in Euler and Schaffrin (1991). The integer least-squares solution $\check{\boldsymbol{a}}_{\mathrm{ILS}}$ is accepted if

$$
\frac{\left\|\hat{\boldsymbol{a}}-\check{\boldsymbol{a}}_{\mathrm{ILS}}\right\|_{\boldsymbol{Q}_{\hat{a}}}^{2}}{\|\hat{\boldsymbol{a}}-\overline{\boldsymbol{a}}\|_{\boldsymbol{Q}_{\hat{\boldsymbol{a}}}}^{2}} \leq \mu,
$$

with the critical value $\mu$. The values produced by the ratio test are within the interval $] 0,1]$. This combined estimation and validation strategy is an integer aperture estimator defined by the aperture pull-in regions

$$
\Omega_{\boldsymbol{z}}=\left\{\boldsymbol{x} \in S_{\boldsymbol{z}} \mid\|\boldsymbol{x}-\boldsymbol{z}\|_{\boldsymbol{Q}_{\hat{a}}}^{2} \leq \mu\|\boldsymbol{x}-\boldsymbol{u}\|_{\boldsymbol{Q}_{\hat{a}}}^{2}, \forall \boldsymbol{u} \in \mathbb{Z}^{n} \backslash \boldsymbol{z}\right\}, \quad \forall \boldsymbol{z} \in \mathbb{Z}^{n}
$$

where $S_{z}$ are the pull-in regions of integer least-squares. Again, the failure rate $P_{\mathrm{F}}$ can be controlled by properly choosing the aperture parameter $\mu$, see Section 5.5. For $\mu \geq 1, \Omega_{z}$ is identical to the respective integer least-squares pull-in region. The regions $\Omega_{z}$ of the ratio test based integer aperture estimator are shown in Figure 3.4 for a two dimensional example. They are constructed as the intersection of hyper ellipsoids. 


\subsubsection{The Projector Test}

The last test of this form that is discussed in this dissertation is the projector test as introduced in Han (1997) and Wang et al. (1998). It is motivated by applying standard methods of hypothesis testing in linear models to the problem of ambiguity validation. Due to the random nature of the involved parameters $\check{\boldsymbol{a}}_{\mathrm{ILS}}$ and $\overline{\boldsymbol{a}}$ and their correlations with $\hat{\boldsymbol{a}}$, the statistical descriptions of such tests are, however, not the usual standard distributions, which has to be considered when determining critical values. The projector test is based on the orthogonal projection of $\hat{\boldsymbol{a}}-\check{\boldsymbol{a}}_{\mathrm{ILS}}$ onto the direction of $\overline{\boldsymbol{a}}-\check{\boldsymbol{a}}_{\mathrm{ILS}}$ in the metric of $\boldsymbol{Q}_{\hat{\boldsymbol{a}}}$. The integer least-squares solution $\check{\boldsymbol{a}}_{\mathrm{ILS}}$ is accepted if

$$
\frac{\left(\overline{\boldsymbol{a}}-\check{\boldsymbol{a}}_{\mathrm{ILS}}\right)^{\mathrm{T}} \boldsymbol{Q}_{\hat{\boldsymbol{a}}}^{-1}\left(\hat{\boldsymbol{a}}-\check{\boldsymbol{a}}_{\mathrm{ILS}}\right)}{\left\|\overline{\boldsymbol{a}}-\check{\boldsymbol{a}}_{\mathrm{ILS}}\right\|_{\boldsymbol{Q}_{\hat{\boldsymbol{a}}}}} \leq \mu,
$$

with the critical value $\mu$. The values produced by the projector test are always non-negative. This combined estimation and validation strategy is an integer aperture estimator defined by the aperture pull-in regions

$$
\Omega_{\boldsymbol{z}}=\left\{\boldsymbol{x} \in S_{\boldsymbol{z}} \mid(\boldsymbol{u}-\boldsymbol{z})^{\mathrm{T}} \boldsymbol{Q}_{\hat{\boldsymbol{a}}}^{-1}(\boldsymbol{x}-\boldsymbol{z}) \leq \mu\|\boldsymbol{u}-\boldsymbol{z}\|_{\boldsymbol{Q}_{\hat{a}}}, \boldsymbol{u}=\underset{\boldsymbol{v} \in \mathbb{Z}^{n} \backslash \boldsymbol{z}}{\operatorname{argmin}}\|\boldsymbol{x}-\boldsymbol{v}\|_{\boldsymbol{Q}_{\hat{a}}}^{2}\right\}, \forall \boldsymbol{z} \in \mathbb{Z}^{n}
$$

where $S_{z}$ are the pull-in regions of integer least-squares. By choosing the aperture parameter $\mu$, the failure rate $P_{\mathrm{F}}$ can be controlled, see Section 5.5. For $\mu \geq \max _{\boldsymbol{x} \in \mathbb{R}^{n}} \frac{1}{2}\left\|\overline{\boldsymbol{x}}-\check{\boldsymbol{x}}_{\mathrm{ILS}}\right\|_{Q_{\hat{a}}}$, where $\check{\boldsymbol{x}}_{\mathrm{ILS}}$ and $\overline{\boldsymbol{x}}$ are the closest and second closest integer grid point of $\boldsymbol{x}, \Omega_{\boldsymbol{z}}$ is identical to the respective integer least-squares pull-in region. The regions of the projector test based integer aperture estimator are shown in Figure 3.4 for a two dimensional example. The aperture pull-in regions are generally non-convex. 


\section{Partial Integer Estimation}

The class of partial integer estimators is a generalization of the class of integer estimators as introduced in Section 3.1 that is capable of resolving an arbitrary subset $\mathcal{I}$ of the integer parameters $a$. The index set $\mathcal{I}$ of a partial integer estimator is deterministic for a given system model. The definition of the class of partial integer estimators is given in Section 4.1 and the quality of the estimates is analyzed in Section 4.2. Three specific estimators including the optimal estimator are discussed in Section 4.3. Criteria and strategies for the problem of how to select the integer transformation $Z$ and index set $\mathcal{I}$ in GNSS applications are presented in Section 4.4. Within this chapter, $\hat{\boldsymbol{a}}$ and $\check{\boldsymbol{a}}$ are used instead of $\hat{\boldsymbol{a}}^{\prime}$ and $\check{\boldsymbol{a}}^{\prime}$ for the estimates after the transformation with $\boldsymbol{Z}$.

\subsection{The Class of Partial Integer Estimators}

The problem is to determine an integer estimate $\check{\boldsymbol{a}}=\mathcal{S}(\hat{\boldsymbol{a}})$ of the parameters $\boldsymbol{a}_{\mathcal{I}}$ from the float solution $\hat{\boldsymbol{a}}$ with the a priori defined deterministic index set $\mathcal{I}$, i.e., to define the mapping $\mathcal{S}(\cdot): \mathbb{R}^{n} \mapsto \mathbb{Z}^{|\mathcal{I}|}$, cf. (2.83). The index set $\mathcal{I}$ can assume any of the $2^{n}-1$ possible non-empty realizations that follow from either including each of the $n$ elements of the parameter vector $\boldsymbol{a}$ or not. Such an estimator can be fully described by the regions $S_{\boldsymbol{z}} \subset \mathbb{R}^{n}, \forall \boldsymbol{z} \in \mathbb{Z}^{|\mathcal{I}|}$, which denote the point set that is mapped to the same integer $z$ via $\mathcal{S}(\cdot)$

$$
S_{\boldsymbol{z}}=\left\{\boldsymbol{x} \in \mathbb{R}^{n} \mid \boldsymbol{z}=\mathcal{S}(\boldsymbol{x})\right\}, \quad \forall \boldsymbol{z} \in \mathbb{Z}^{|\mathcal{I}|} .
$$

The partial integer estimator (2.83) corresponding to these regions can be explicitly written as

$$
\check{\boldsymbol{a}}=\sum_{\boldsymbol{z} \in \mathbb{Z}^{|\mathcal{I}|}} s_{\boldsymbol{z}}(\hat{\boldsymbol{a}}) \boldsymbol{z}
$$

with the indicator function

$$
s_{\boldsymbol{z}}(\hat{\boldsymbol{a}})= \begin{cases}1 & \text { if } \hat{\boldsymbol{a}} \in S_{\boldsymbol{z}} \\ 0 & \text { else. }\end{cases}
$$

We notice that the definition of a partial integer estimator is very similar to the one of an integer estimator in Section 3.1, but with the possible output values being in the $|\mathcal{I}|$ dimensional space of integers $\mathbb{Z}^{|\mathcal{I}|}$ instead of in the $n$ dimensional space of integers $\mathbb{Z}^{n}$. This is of importance when formulating the constraints that have to be imposed on the construction of the regions $S_{z}$, which are then slightly different. The following three properties have to be met by the regions $S_{z}$ defining a partial integer estimator with a given non-empty subset $\mathcal{I}$ (Brack 2016a):

$$
\begin{aligned}
& \text { (i) } \bigcup_{\boldsymbol{z} \in \mathbb{Z}|\mathcal{I}|} S_{\boldsymbol{z}}=\mathbb{R}^{n} \\
& \text { (ii) } \operatorname{int}\left(S_{\boldsymbol{z}}\right) \cap \operatorname{int}\left(S_{\boldsymbol{u}}\right)=\emptyset, \quad \forall \boldsymbol{z}, \boldsymbol{u} \in \mathbb{Z}^{|\mathcal{I}|}, \boldsymbol{z} \neq \boldsymbol{u} \\
& \text { (iii) } S_{\boldsymbol{v}_{\mathcal{I}}}=S_{\mathbf{0}}+\boldsymbol{v}, \quad \forall \boldsymbol{v} \in \mathbb{Z}^{n} .
\end{aligned}
$$

We note that in condition $($ iii $) \boldsymbol{v}$ is an $n$ dimensional integer vector, but the region $S_{\boldsymbol{v}_{\mathcal{I}}}$ is only linked to the elements $\boldsymbol{v}_{\mathcal{I}}$ that correspond to the subset $\mathcal{I}$. 
The first two conditions state that the regions $S_{\boldsymbol{z}}$ have to cover the space $\mathbb{R}^{n}$ without gaps and overlaps, meaning that the (partial) integer mapping $\mathcal{S}(\cdot)$ leads to a unique $|\mathcal{I}|$ dimensional integer estimate for any float solution $\hat{\boldsymbol{a}} \in \mathbb{R}^{n}$. The third condition states that if the float solution $\hat{\boldsymbol{a}}$ is shifted by an arbitrary integer vector $\boldsymbol{v} \in \mathbb{Z}^{n}$, then the integer estimate for the subset $\mathcal{I}$ has to be shifted by $\boldsymbol{v}_{\mathcal{I}}$. This implies that the regions $S_{\boldsymbol{z}}$ are translated copies of each other for any integer shift in the directions that correspond to the subset $\mathcal{I}$, and that the regions $S_{z}$ are invariant for any integer shift in the directions that correspond to the subset $\overline{\mathcal{I}}$. In other words, if any entry of the float solution $\hat{\boldsymbol{a}}$ that is to be resolved is shifted by an arbitrary integer, then the integer solution is shifted by the same amount. On the other hand, if any entry of the float solution $\hat{\boldsymbol{a}}$ that is not resolved is shifted by an arbitrary integer, this must not affect the integer solution of the subset to be resolved. For any non-empty index set $\mathcal{I}$ with $|\mathcal{I}|<n$, each region $S_{\boldsymbol{z}}$ is linked to a unique $|\mathcal{I}|$ dimensional integer vector, but generally contains an infinite number of $n$ dimensional integer grid points-except for the case that all integer grid points lie on the boundaries of the regions $S_{z}$. This means that the regions $S_{z}$ for $|\mathcal{I}|<n$ are not pull-in regions, but rather decision regions. It can easily be seen that for $\mathcal{I}=\{1, \ldots, n\}$ the properties in (4.4) are identical to the ones of an integer estimator in (3.4).

\subsection{Quality of the Estimates}

With $p_{\hat{\boldsymbol{a}}}(\boldsymbol{x})$ the Gaussian pdf of the float solution $\hat{\boldsymbol{a}}$, the pmf of $\check{\boldsymbol{a}}$ for any non-empty index set $\mathcal{I}$ and an estimator with the decision regions $S_{z}$ follows as

$$
P(\check{\boldsymbol{a}}=\boldsymbol{z})=P\left(\hat{\boldsymbol{a}} \in S_{\boldsymbol{z}}\right)=\int_{S_{\boldsymbol{z}}} p_{\hat{\boldsymbol{a}}}(\boldsymbol{x}) \mathrm{d} \boldsymbol{x}, \quad \forall \boldsymbol{z} \in \mathbb{Z}^{|\mathcal{I}|} .
$$

The probability of correctly resolving the given subset $\mathcal{I}$ of integer parameters is given by $P_{\mathrm{S}}=$ $P\left(\check{\boldsymbol{a}}=\boldsymbol{a}_{\mathcal{I}}\right)$. We have to keep in mind that when using the term success rate for $P_{\mathrm{S}}$ for a partial integer estimator, it refers to correctly estimating a specific subset $\mathcal{I}$ of the integer parameters $a$ and is therefore generally a weaker measure than the probability of correctly resolving the full set of integer parameters as defined in (3.1.1) for the class of integer estimators. The probability of an incorrect integer estimate $\check{\boldsymbol{a}}$ follows as $P_{\mathrm{F}}=1-P_{\mathrm{S}}$.

In order to derive the pdf of the partially fixed estimates $\check{b}$ of the real valued parameters as defined in (2.84), we make use of the following invertible parameter transformation

$$
\left[\begin{array}{c}
\check{b} \\
\hat{\boldsymbol{a}}_{\overline{\mathcal{I}}} \\
\hat{\boldsymbol{a}}_{\mathcal{I}} \\
\check{\boldsymbol{a}}
\end{array}\right]=\underbrace{\left[\begin{array}{cccc}
\boldsymbol{I} & \mathbf{0} & -\boldsymbol{Q}_{\hat{\boldsymbol{b}} \hat{\boldsymbol{a}}_{\mathcal{I}}} \boldsymbol{Q}_{\hat{\boldsymbol{a}}_{\mathcal{I}}}^{-1} & \boldsymbol{Q}_{\hat{b} \hat{\boldsymbol{a}}_{\mathcal{I}}} \boldsymbol{Q}_{\hat{\boldsymbol{a}}_{\mathcal{I}}}^{-1} \\
\mathbf{0} & \boldsymbol{I} & \mathbf{0} & \mathbf{0} \\
\mathbf{0} & \mathbf{0} & \boldsymbol{I} & \mathbf{0} \\
\mathbf{0} & \mathbf{0} & \mathbf{0} & \boldsymbol{I}
\end{array}\right]}_{T}\left[\begin{array}{c}
\hat{\boldsymbol{b}} \\
\hat{\boldsymbol{a}}_{\overline{\mathcal{I}}} \\
\hat{\boldsymbol{a}}_{\mathcal{I}} \\
\check{\boldsymbol{a}}
\end{array}\right] .
$$

From the change of variables theorem follows that if two continuous random variables $\boldsymbol{r}$ and $\boldsymbol{q}$ are related via $\boldsymbol{r}=\boldsymbol{T} \boldsymbol{q}$, with $\boldsymbol{T}$ invertible, then $p_{\boldsymbol{r}}(\boldsymbol{x})=\frac{1}{|\operatorname{det}(\boldsymbol{T})|} p_{\boldsymbol{q}}\left(\boldsymbol{T}^{-1} \boldsymbol{x}\right)$. The first part of Theorem 2 in Teunissen (2002) states that for an integer estimator $p_{\hat{\boldsymbol{b}}, \hat{\boldsymbol{a}}, \check{\boldsymbol{a}}}(\boldsymbol{w}, \boldsymbol{x}, \boldsymbol{z})=p_{\hat{\boldsymbol{b}}, \hat{\boldsymbol{a}}}(\boldsymbol{w}, \boldsymbol{x}) s_{\boldsymbol{z}}(\boldsymbol{x})$, where $p_{\hat{\boldsymbol{b}}, \hat{\boldsymbol{a}}}(\boldsymbol{w}, \boldsymbol{x})$ is jointly Gaussian and $\check{\boldsymbol{a}} \in \mathbb{Z}^{n}$. This theorem also applies to a partial integer estimator with $\check{\boldsymbol{a}} \in \mathbb{Z}^{|\mathcal{I}|}$. The pdf of the transformed parameter vector in (4.6) is thus $p_{\check{\boldsymbol{b}}, \hat{\boldsymbol{a}}, \check{\boldsymbol{a}}}(\boldsymbol{\beta}, \boldsymbol{x}, \boldsymbol{z})=$ $p_{\hat{\boldsymbol{b}}, \hat{\boldsymbol{a}}}\left(\boldsymbol{\beta}+\boldsymbol{Q}_{\hat{\boldsymbol{b}} \hat{\boldsymbol{a}}_{\mathcal{I}}} \boldsymbol{Q}_{\hat{\boldsymbol{a}}_{\mathcal{I}}}^{-1}\left(\boldsymbol{x}_{\mathcal{I}}-\boldsymbol{z}\right), \boldsymbol{x}\right) s_{\boldsymbol{z}}(\boldsymbol{x})$. With the above defined properties of the regions $S_{\boldsymbol{z}}$, the 
pdf of $\check{\boldsymbol{b}}$ can now be expressed as

$$
\begin{aligned}
p_{\check{\boldsymbol{b}}}(\boldsymbol{\beta}) & =\sum_{\boldsymbol{z} \in \mathbb{Z}|\mathcal{I}|} \int_{S_{\boldsymbol{z}}} p_{\hat{\boldsymbol{b}}, \hat{\boldsymbol{a}}}\left(\boldsymbol{\beta}+\boldsymbol{Q}_{\hat{\boldsymbol{b}} \hat{\boldsymbol{a}}_{\mathcal{I}}} \boldsymbol{Q}_{\hat{\boldsymbol{a}}_{\mathcal{I}}}^{-1}\left(\boldsymbol{x}_{\mathcal{I}}-\boldsymbol{z}\right), \boldsymbol{x}\right) \mathrm{d} \boldsymbol{x} \\
& =\sum_{\boldsymbol{z} \in \mathbb{Z}|\mathcal{I}|} \int_{S_{\boldsymbol{z}}} p_{\hat{\boldsymbol{b}}, \hat{\boldsymbol{a}}_{\overline{\mathcal{I}}} \mid \hat{\boldsymbol{a}}_{\mathcal{I}}}\left(\boldsymbol{\beta}+\boldsymbol{Q}_{\hat{\boldsymbol{b}} \hat{\boldsymbol{a}}_{\mathcal{I}}} \boldsymbol{Q}_{\hat{\boldsymbol{a}}_{\mathcal{I}}}^{-1}\left(\boldsymbol{x}_{\mathcal{I}}-\boldsymbol{z}\right), \boldsymbol{x}_{\overline{\mathcal{I}}} \mid \boldsymbol{x}_{\mathcal{I}}\right) p_{\hat{\boldsymbol{a}}_{\mathcal{I}}}\left(\boldsymbol{x}_{\mathcal{I}}\right) \mathrm{d} \boldsymbol{x},
\end{aligned}
$$

where the second equality follows from the definition of a conditional probability distribution. Unfortunately, expression (4.7) is not very intuitive. In order to gain some deeper insight we analyze two special cases in more detail in the following. As a first case we assume that the integer estimate $\check{\boldsymbol{a}}$ of $\boldsymbol{a}_{\mathcal{I}}$ is computed only as a function of $\hat{\boldsymbol{a}}_{\mathcal{I}}$ instead of the full float solution $\hat{\boldsymbol{a}}$. Let $\tilde{\mathcal{S}}: \mathbb{R}^{|\mathcal{I}|} \mapsto \mathbb{Z}^{|\mathcal{I}|}$ be defined such that $\tilde{\mathcal{S}}\left(\boldsymbol{x}_{\mathcal{I}}\right)=\mathcal{S}(\boldsymbol{x}), \forall \boldsymbol{x} \in \mathbb{R}^{n}$, and let $\tilde{S}_{\boldsymbol{z}} \subset \mathbb{R}^{|\mathcal{I}|}, \forall \boldsymbol{z} \in \mathbb{Z}^{|\mathcal{I}|}$, denote the corresponding pull-in regions. The pdf of $\breve{b}$ can then be written as

$$
\begin{aligned}
p_{\check{\boldsymbol{b}}}(\boldsymbol{\beta}) & =\sum_{\boldsymbol{z} \in \mathbb{Z}|\mathcal{I}|} \int_{\tilde{S}_{\boldsymbol{z}}} \int_{\mathbb{R}^{n-|\mathcal{I}|}} p_{\hat{\boldsymbol{b}}, \hat{\boldsymbol{a}}_{\overline{\mathcal{I}}} \mid \hat{\boldsymbol{a}}_{\mathcal{I}}}\left(\boldsymbol{\beta}+\boldsymbol{Q}_{\hat{\boldsymbol{b}} \hat{\boldsymbol{a}}_{\mathcal{I}}} \boldsymbol{Q}_{\hat{\boldsymbol{a}}_{\mathcal{I}}}^{-1}\left(\boldsymbol{x}_{\mathcal{I}}-\boldsymbol{z}\right), \boldsymbol{x}_{\overline{\mathcal{I}}} \mid \boldsymbol{x}_{\mathcal{I}}\right) p_{\hat{\boldsymbol{a}}_{\mathcal{I}}}\left(\boldsymbol{x}_{\mathcal{I}}\right) \mathrm{d} \boldsymbol{x}_{\overline{\mathcal{I}}} \mathrm{d} \boldsymbol{x}_{\mathcal{I}} \\
& =\sum_{\boldsymbol{z} \in \mathbb{Z}|\mathcal{I}|} \int_{\tilde{S}_{\boldsymbol{z}}} p_{\hat{\boldsymbol{b}} \mid \hat{\boldsymbol{a}}_{\mathcal{I}}}\left(\boldsymbol{\beta}+\boldsymbol{Q}_{\hat{\boldsymbol{b}}_{\hat{\boldsymbol{a}}_{\mathcal{I}}}} \boldsymbol{Q}_{\hat{\boldsymbol{a}}_{\mathcal{I}}}^{-1}\left(\boldsymbol{x}_{\mathcal{I}}-\boldsymbol{z}\right) \mid \boldsymbol{x}_{\mathcal{I}}\right) p_{\hat{\boldsymbol{a}}_{\mathcal{I}}}\left(\boldsymbol{x}_{\mathcal{I}}\right) \mathrm{d} \boldsymbol{x}_{\mathcal{I}} \\
& =\sum_{\boldsymbol{z} \in \mathbb{Z}|\mathcal{I}|} p_{\hat{\boldsymbol{b}} \mid \hat{\boldsymbol{a}}_{\mathcal{I}}}(\boldsymbol{\beta} \mid \boldsymbol{z}) P(\check{\boldsymbol{a}}=\boldsymbol{z})
\end{aligned}
$$

The third equality follows from $p_{\hat{\boldsymbol{b}} \mid \hat{\boldsymbol{a}}_{\mathcal{I}}}\left(\boldsymbol{\beta}+\boldsymbol{Q}_{\hat{\boldsymbol{b}} \hat{\boldsymbol{a}}_{\mathcal{I}}} \boldsymbol{Q}_{\hat{\boldsymbol{a}}_{\mathcal{I}}}^{-1}\left(\boldsymbol{x}_{\mathcal{I}}-\boldsymbol{z}\right) \mid \boldsymbol{x}_{\mathcal{I}}\right)=p_{\hat{\boldsymbol{b}} \mid \hat{\boldsymbol{a}}_{\mathcal{I}}}(\boldsymbol{\beta} \mid \boldsymbol{z})$ and $\int_{\tilde{S}_{\boldsymbol{z}}} p_{\hat{\boldsymbol{a}}_{\mathcal{I}}}\left(\boldsymbol{x}_{\mathcal{I}}\right) \mathrm{d} \boldsymbol{x}_{\mathcal{I}}=P(\check{\boldsymbol{a}}=\boldsymbol{z})$. The conditional Gaussian pdf $p_{\hat{\boldsymbol{b}} \mid \hat{\boldsymbol{a}}_{\mathcal{I}}}(\boldsymbol{\beta} \mid \boldsymbol{z})$ is characterized by the conditional mean $\boldsymbol{b}-\boldsymbol{Q}_{\hat{\boldsymbol{b}} \hat{\boldsymbol{a}}_{\mathcal{I}}} \boldsymbol{Q}_{\hat{\boldsymbol{a}}_{\mathcal{I}}}^{-1}(\boldsymbol{a}-\boldsymbol{z})$ and the conditional covariance matrix

$$
Q_{\hat{b} \mid \hat{a}_{\mathcal{I}}}=Q_{\hat{b}}-Q_{\hat{b}_{\hat{I}_{\mathcal{I}}}} Q_{\hat{a}_{\mathcal{I}}}^{-1} Q_{\hat{a}_{\mathcal{I}} \hat{b}}
$$

Since an estimator of this form can also be interpreted in the way that an integer estimator is applied to an observation model in which $\boldsymbol{a}_{\overline{\mathcal{I}}}$ belongs to the real valued parameter vector, the final expression (4.8) could directly be expected from (3.6).

As a second case, we study (4.7) for a very high probability of correct estimates $\check{a}$. For $P\left(\check{\boldsymbol{a}}=\boldsymbol{a}_{\mathcal{I}}\right) \rightarrow 1$, the integer estimate $\check{\boldsymbol{a}}$ becomes deterministic, so that (4.7) becomes

$$
\begin{aligned}
p_{\check{\boldsymbol{b}}}(\boldsymbol{\beta}) & =\int_{\mathbb{R}^{n}} p_{\hat{\boldsymbol{b}}, \hat{\boldsymbol{a}}}\left(\boldsymbol{\beta}+\boldsymbol{Q}_{\hat{\boldsymbol{b}} \hat{\boldsymbol{a}}_{\mathcal{I}}} \boldsymbol{Q}_{\hat{\boldsymbol{a}}_{\mathcal{I}}}^{-1}\left(\boldsymbol{x}_{\mathcal{I}}-\boldsymbol{a}_{\mathcal{I}}\right), \boldsymbol{x}\right) \mathrm{d} \boldsymbol{x} \\
& =p_{\hat{\boldsymbol{b}} \mid \hat{\boldsymbol{a}}_{\mathcal{I}}}\left(\boldsymbol{\beta} \mid \boldsymbol{a}_{\mathcal{I}}\right)
\end{aligned}
$$

where for the second equality the same relation as in the final step of (4.8) is used. If the success rate of a partial integer estimator is sufficiently close to one, we can therefore make use of the conditional Gaussian distribution with mean value $\boldsymbol{b}$ and covariance matrix $\boldsymbol{Q}_{\hat{\boldsymbol{b}} \mid \hat{\boldsymbol{a}}_{\mathcal{I}}}$, see (4.9), to describe the stochastic properties of $\breve{b}$.

With the deterministic index set $\mathcal{I}$ of a partial integer estimator, the estimate $\check{b}$ is unbiased according to (2.85), if $\mathrm{E}[\check{\boldsymbol{a}}]=\boldsymbol{a}_{\mathcal{I}}$, i.e., if the integer estimate $\check{\boldsymbol{a}}$ itself is unbiased. This is the case if $S_{\boldsymbol{a}_{\mathcal{I}}+\boldsymbol{z}}$ is the point reflection across $\boldsymbol{a}$ of $S_{\boldsymbol{a}_{\mathcal{I}} \boldsymbol{z}}, \forall \boldsymbol{z} \in \mathbb{Z}^{|\mathcal{I}|}$. With the properties (4.4) this is equivalent to $S_{\boldsymbol{z}}$ being symmetric with respect to $\boldsymbol{v} \in \mathbb{Z}^{n}$ with $\boldsymbol{v}_{\mathcal{I}}=\boldsymbol{z}$. 


\subsection{Examples of Partial Integer Estimators}

Three specific realizations of partial integer estimators are presented in the following. The first two make use of standard methods from integer estimation and adapt them so as to be capable of estimating a subset of integers. They are explicitly formulated for integer least-squares, but could also be based on integer bootstrapping, element-wise rounding, or any other integer estimator as defined in Section 3.1. The third estimator is the one that leads to the highest possible probability of correct integer estimates of $\boldsymbol{a}_{\mathcal{I}}$ for a given index set $\mathcal{I}$. Implementation aspects of the optimal estimator are discussed.

\subsubsection{Partial Integer Least-Squares}

The probably most intuitive approach for estimating a given subset of integers as defined by the index set $\mathcal{I}$ is to only consider the part $\hat{\boldsymbol{a}}_{\mathcal{I}}$ of the float solution $\hat{\boldsymbol{a}}$, which lies in the subspace that corresponds to the parameters that are to be resolved as integers, and to apply a standard integer estimator to $\hat{\boldsymbol{a}}_{\mathcal{I}}$. This implies that only the information $\hat{\boldsymbol{a}}_{\mathcal{I}}$ is used for computing the integer estimate whereas $\hat{\boldsymbol{a}}_{\overline{\mathcal{I}}}$ is not, meaning that the parameters $\boldsymbol{a}_{\overline{\mathcal{I}}}$ that are not resolved as integers are treated as if they were conceptually real valued, i.e., $\boldsymbol{a}_{\overline{\mathcal{I}}}$ is treated as if it were a part of $\boldsymbol{b}$. According to the system model (2.75) and (2.78), the parameters $\boldsymbol{a}_{\overline{\mathcal{I}}}$ are, however, integers, which is completely ignored by this scheme. Essentially, this estimator considers a reduced $|\mathcal{I}|$ dimensional space and resolves the full vector of integer parameters-which is then of dimension $|\mathcal{I}|$-in that space. For integer least-squares, the corresponding integer estimate is given by

$$
\check{\boldsymbol{a}}_{\mathrm{parILS}}=\underset{\boldsymbol{z} \in \mathbb{Z}^{|\mathcal{I}|}}{\operatorname{argmin}}\left\|\hat{\boldsymbol{a}}_{\mathcal{I}}-\boldsymbol{z}\right\|_{\boldsymbol{Q}_{\hat{\boldsymbol{a}}_{\mathcal{I}}}}^{2},
$$

where $\boldsymbol{Q}_{\hat{\boldsymbol{a}}_{\mathcal{I}}}$ follows from selecting the entries that correspond to $\hat{\boldsymbol{a}}_{\mathcal{I}}$ from $\boldsymbol{Q}_{\hat{\boldsymbol{a}}}$. The decision regions $S_{z}$ of this partial integer least-squares estimator are given by

$$
S_{\boldsymbol{z}}=\left\{\boldsymbol{x} \in \mathbb{R}^{n} \mid\left\|\boldsymbol{x}_{\mathcal{I}}-\boldsymbol{z}\right\|_{\boldsymbol{Q}_{\hat{a}_{\mathcal{I}}}}^{2} \leq\left\|\boldsymbol{x}_{\mathcal{I}}-\boldsymbol{u}\right\|_{\boldsymbol{Q}_{\hat{a}_{\mathcal{I}}}}^{2}, \forall \boldsymbol{u} \in \mathbb{Z}^{|\mathcal{I}|}\right\}, \quad \forall \boldsymbol{z} \in \mathbb{Z}^{|\mathcal{I}|}
$$

This estimator can easily be shown to fulfill the constraints in (4.4). It produces a unique $|\mathcal{I}|$ dimensional integer vector for any float solution $\hat{\boldsymbol{a}} \in \mathbb{R}^{n}$ that is not on the boundary between two decision regions. With $\operatorname{argmin}_{\boldsymbol{z} \in \mathbb{Z}|\mathcal{I}|}\left\|\hat{\boldsymbol{a}}_{\mathcal{I}}-\boldsymbol{u}-\boldsymbol{z}\right\|_{\boldsymbol{Q}_{\hat{\boldsymbol{a}}_{\mathcal{I}}}}^{2}+\boldsymbol{u}=\operatorname{argmin}_{\boldsymbol{z} \in \mathbb{Z}|\mathcal{I}|}\left\|\hat{\boldsymbol{a}}_{\mathcal{I}}-\boldsymbol{z}\right\|_{\boldsymbol{Q}_{\hat{\boldsymbol{a}}_{\mathcal{I}}}}^{2}, \forall \boldsymbol{u} \in \mathbb{Z}^{|\mathcal{I}|}$, also the third property holds, since an arbitrary shift of $\hat{\boldsymbol{a}}_{\overline{\mathcal{I}}}$ has no impact on the integer solution.

As integer least-squares is optimal in the sense of maximizing the success rate for estimating the full vector of integer parameters, this estimator seems to be a reasonable choice. It does, however, completely neglect the fact that the parameters $\boldsymbol{a}_{\overline{\mathcal{I}}}$ are integers as well, since the computation of the integer solution (4.11) is independent of $\hat{\boldsymbol{a}}_{\overline{\mathcal{I}}}$, and is therefore certainly not optimal.

The evaluation of success rates for such schemes that apply an integer estimator to the partial float solution $\hat{\boldsymbol{a}}_{\mathcal{I}}$ is carried out analogously to the standard integer estimators. The success rate of partial integer bootstrapping, which follows from replacing integer least-squares with integer bootstrapping in (4.11), can thus be computed in closed form. Depending on the choice of the parameterization of the float estimates via $Z$, it might therefore be useful to consider an additional decorrelation transformation $Z^{\prime} \in \mathbb{Z}^{|\mathcal{I}| \times|\mathcal{I}|}$ for $\hat{\boldsymbol{a}}_{\mathcal{I}}$ and to compute the integer estimates or success rates in the decorrelated space characterized by the covariance matrix $\boldsymbol{Z}^{\prime} \boldsymbol{Q}_{\hat{\boldsymbol{a}}_{\mathcal{I}}} \boldsymbol{Z}^{\prime \mathrm{T}}$. The integer estimate can then be transformed back via $Z^{\prime-1}$. It is important to note the different purposes of these two integer transformations. The rows of the matrix $Z$ in combination with the index 

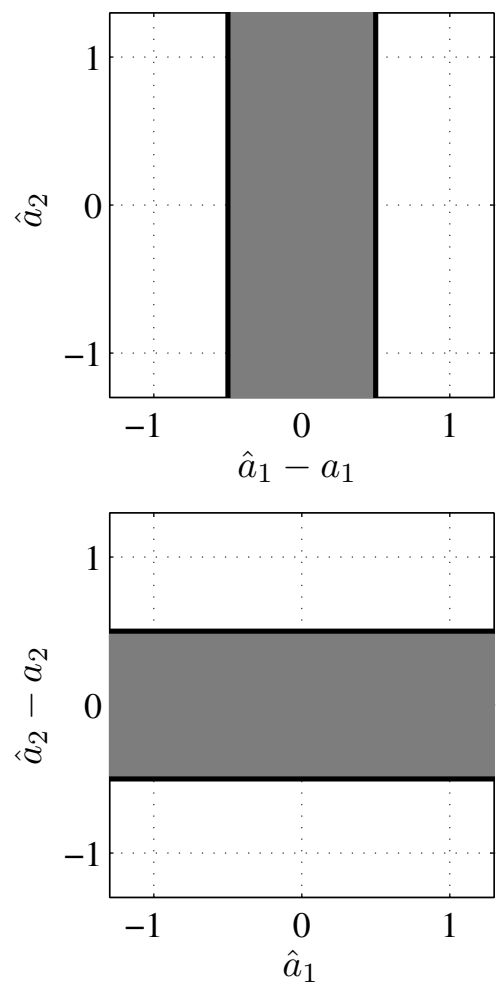

(a) Partial integer least-squares
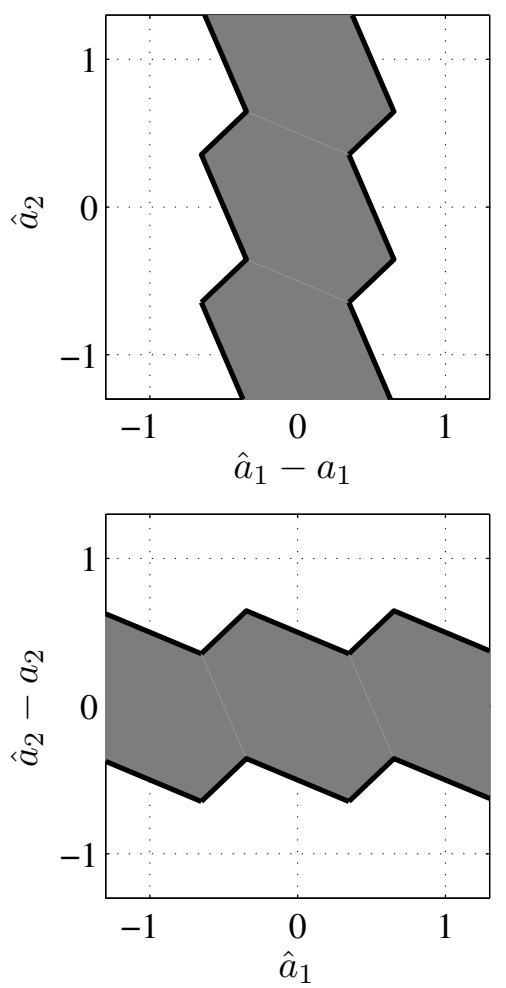

(b) Full integer least-squares + selection
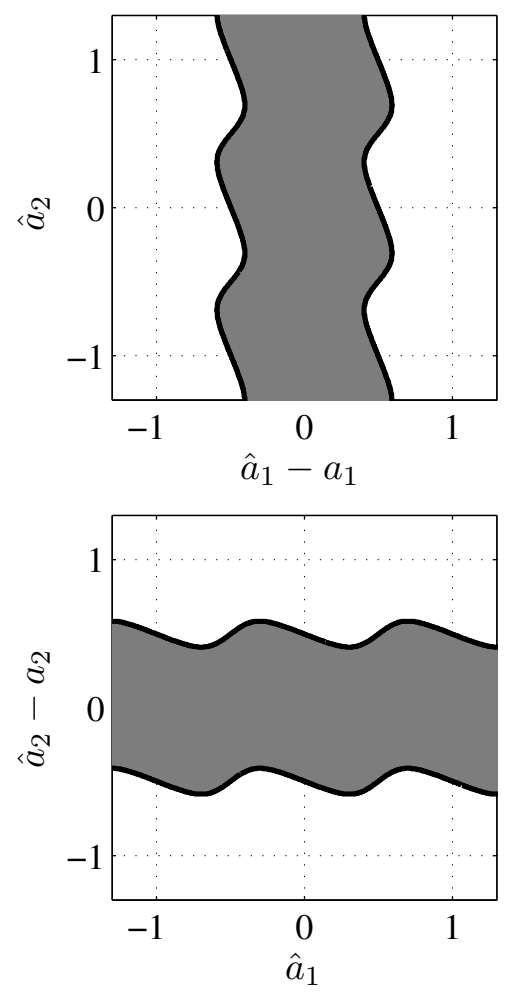

(c) Optimal partial integer estimation

Figure 4.1: Regions $S_{z}$ of partial integer estimators for a two dimensional example with low correlation for $\mathcal{I}=\{1\}$ in the first row and $\mathcal{I}=\{2\}$ in the second row. The regions that lead to correct integer estimates are marked in gray.

set $\mathcal{I}$ define the linear combinations of the integer parameters that are resolved, whereas the sole purpose of matrix $\boldsymbol{Z}^{\prime}$ is to improve the efficiency or success rate of the estimator, without changing the actual problem. The decision regions $S_{z}$ of partial integer least-squares are illustrated for a two dimensional example with low correlation in Figure 4.1 and with high correlation in Figure 4.2 for $\mathcal{I}=\{1\}$ and $\mathcal{I}=\{2\}$.

\subsubsection{Full Integer Least-Squares + Selection}

A different approach to make use of the class of integer estimators for estimating the subset $\mathcal{I}$ of integer parameters is to apply an integer estimator to the full $n$ dimensional float solution $\hat{\boldsymbol{a}}$, and to subsequently select those entries from the resulting $n$ dimensional integer estimate that correspond to the subset $\mathcal{I}$. For integer least-squares, the integer estimates are given by

$$
\check{\boldsymbol{a}}_{\mathrm{fullILS}}=\boldsymbol{\Gamma}_{\mathcal{I}} \check{\boldsymbol{a}}_{\mathrm{ILS}}, \quad \text { with } \quad \check{\boldsymbol{a}}_{\mathrm{ILS}}=\underset{\boldsymbol{v} \in \mathbb{Z}^{n}}{\operatorname{argmin}}\|\hat{\boldsymbol{a}}-\boldsymbol{v}\|_{\boldsymbol{Q}_{\hat{\boldsymbol{a}}}}^{2},
$$

where the multiplication of a vector $\boldsymbol{x} \in \mathbb{R}^{n}$ with $\boldsymbol{\Gamma}_{\mathcal{I}} \in\{0,1\}^{|\mathcal{I}| \times n}$ selects $\boldsymbol{x}_{\mathcal{I}}$ from $\boldsymbol{x}$. Accordingly, the decision regions $S_{z}$ of full integer least-squares followed by a selection of entries are given by the union of all pull-in regions of the full $n$ dimensional integer least-squares estimator that correspond to an integer vector $\boldsymbol{v} \in \mathbb{Z}^{n}$ with $\boldsymbol{v}_{\mathcal{I}}=\boldsymbol{z}$

$$
S_{\boldsymbol{z}}=\bigcup_{\boldsymbol{v} \in \mathbb{Z}^{n} \mid \boldsymbol{v}_{\mathcal{I}}=\boldsymbol{z}}\left\{\boldsymbol{x} \in \mathbb{R}^{n} \mid\|\boldsymbol{x}-\boldsymbol{v}\|_{\boldsymbol{Q}_{\hat{\boldsymbol{a}}}}^{2} \leq\|\boldsymbol{x}-\boldsymbol{u}\|_{\boldsymbol{Q}_{\hat{a}}}^{2}, \forall \boldsymbol{u} \in \mathbb{Z}^{n}\right\}, \quad \forall \boldsymbol{z} \in \mathbb{Z}^{|\mathcal{I}|}
$$



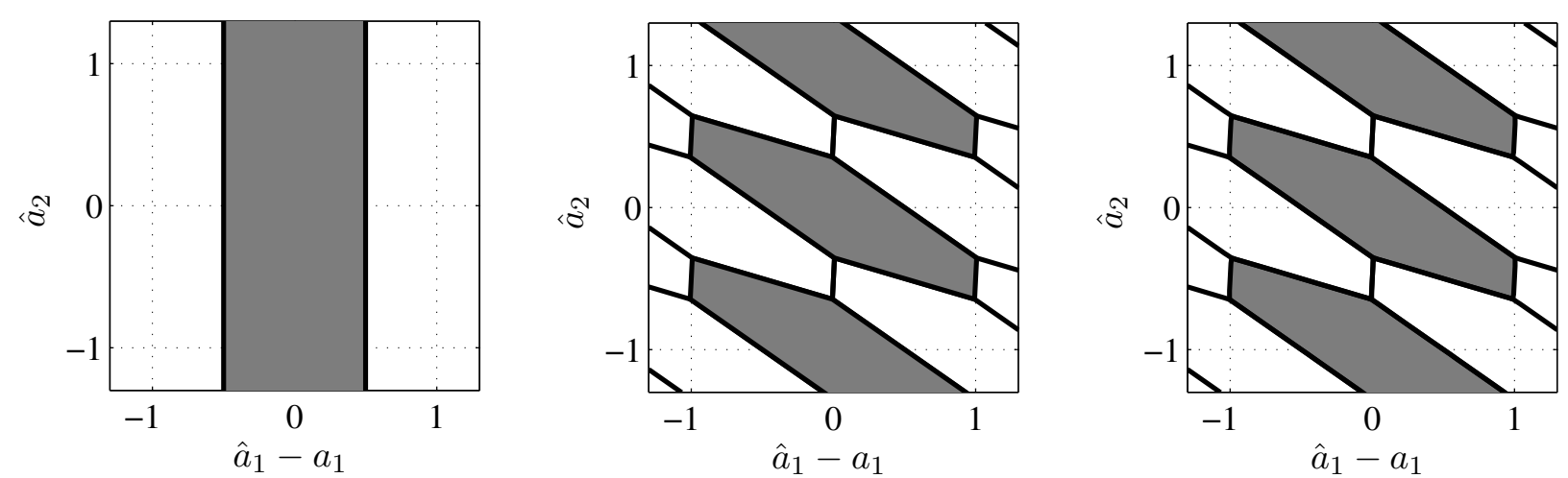

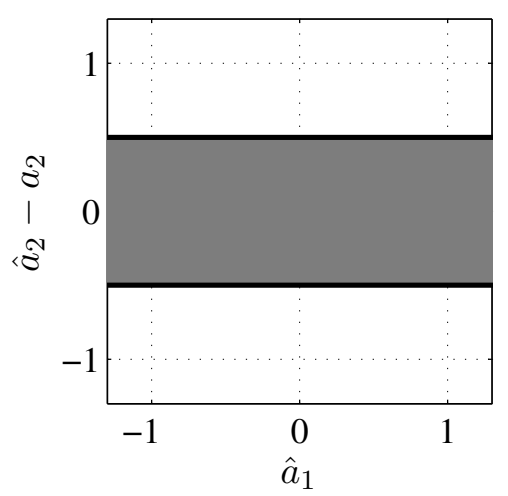

(a) Partial integer least-squares

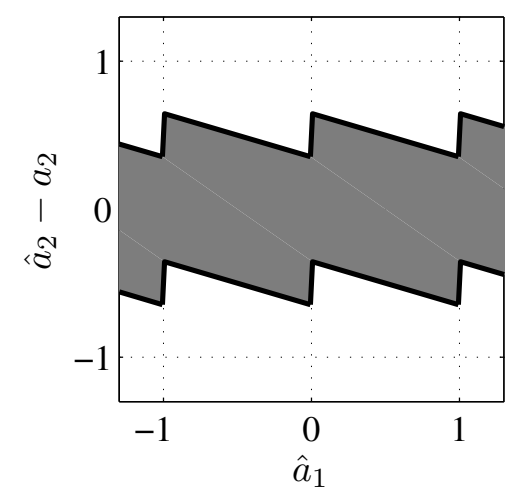

(b) Full integer least-squares + selection

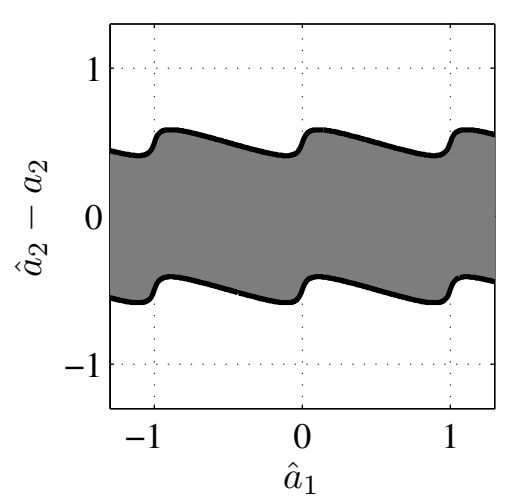

(c) Optimal partial integer estimation

Figure 4.2: Regions $S_{z}$ of partial integer estimators for a two dimensional example with high correlation for $\mathcal{I}=\{1\}$ in the first row and $\mathcal{I}=\{2\}$ in the second row. The regions that lead to correct integer estimates are marked in gray. 
This estimator produces a unique $|\mathcal{I}|$ dimensional integer vector for any float solution $\hat{\boldsymbol{a}} \in \mathbb{R}^{n}$, and thus meets the first two criteria form (4.4). Further, with $\operatorname{argmin}_{\boldsymbol{v} \in \mathbb{Z}^{n}}\|\hat{\boldsymbol{a}}-\boldsymbol{u}-\boldsymbol{v}\|_{\boldsymbol{Q}_{\hat{\boldsymbol{a}}}}^{2}+\boldsymbol{u}=$ $\operatorname{argmin}_{\boldsymbol{v} \in \mathbb{Z}^{n}}\|\hat{\boldsymbol{a}}-\boldsymbol{v}\|_{\boldsymbol{Q}_{\hat{a}}}^{2}, \forall \boldsymbol{u} \in \mathbb{Z}^{n}$, also the third condition is met when selecting entries from the full integer least-squares solution.

This estimator clearly takes the integer property of $\boldsymbol{a}_{\overline{\mathcal{I}}}$ into account, but integer least-squares in combination with a selection of entries of the resulting $n$ dimensional integer solution is no longer optimal.

The success rate of full integer least-squares followed by a selection of entries cannot be easily evaluated, since for $|\mathcal{I}|<n$ there is an infinite number of $n$ dimensional integer solutions that lead to correct integer estimates $\check{\boldsymbol{a}}_{\text {fullILS }}$, i.e., the region of integration $S_{\boldsymbol{a}_{\mathcal{I}}}$ consists of infinitely many pull-in regions of volume one. This also implies that the evaluation of bounds becomes more complicated. A very simple but also not very useful upper bound for the failure rate $P\left(\check{\boldsymbol{a}}_{\text {fullILS }} \neq \boldsymbol{a}_{\mathcal{I}}\right)$ is given by the probability that the full integer least-squares solution $\check{a}_{\mathrm{ILS}}$ is incorrect, which can again be bounded by, e.g., the integer bootstrapping failure rate. Depending on the chosen parameterization via $Z$, it might again be useful to compute the $n$ dimensional integer estimate after an additional decorrelation transformation that is inverted before selecting the entries corresponding to $\mathcal{I}$.

It is noted that if no additional transformations are applied, the estimation schemes (4.11) and (4.13) are identical if integer least-squares is replaced by element-wise rounding, and-depending on the order-may be identical for integer bootstrapping. The decision regions $S_{z}$ of full integer least-squares + selection are illustrated in Figure 4.1 and 4.2 for two different two dimensional examples for $\mathcal{I}=\{1\}$ and $\mathcal{I}=\{2\}$. In the second example, a substantial correlation is present between the two components, which causes the regions $S_{z}$ for $\mathcal{I}=\{1\}$ to be unconnected.

\subsubsection{Optimal Partial Integer Estimation}

Depending on the index set $\mathcal{I}$ and the covariance matrix $\boldsymbol{Q}_{\hat{a}}$ either of the two above introduced integer least-squares based estimators may be the better choice in terms of the probability of correct integer estimates. It is, however, possible to formulate the optimal estimator, which resolves a given subset $\mathcal{I}$ of the integer parameters $\boldsymbol{a}$ with the highest possible probability of correct integer estimates $P\left(\check{\boldsymbol{a}}=\boldsymbol{a}_{\mathcal{I}}\right)$ within the class of partial integer estimators as defined in (4.2), see Brack (2016a). Let the integer estimates $\check{\boldsymbol{a}}_{\text {opt }}$ of $\boldsymbol{a}_{\mathcal{I}}$ be given by

$$
\check{\boldsymbol{a}}_{\mathrm{opt}}=\underset{\boldsymbol{z} \in \mathbb{Z}^{|\mathcal{I}|}}{\operatorname{argmax}} \sum_{\boldsymbol{v} \in \mathbb{Z}^{n} \mid \boldsymbol{v}_{\mathcal{I}}=\boldsymbol{z}} \exp \left(-\frac{1}{2}\|\hat{\boldsymbol{a}}-\boldsymbol{v}\|_{\boldsymbol{Q}_{\hat{\boldsymbol{a}}}}^{2}\right)
$$

where the float solution $\hat{\boldsymbol{a}}$ follows the Gaussian distribution $\mathcal{G}\left(\boldsymbol{a}, \boldsymbol{Q}_{\hat{\boldsymbol{a}}}\right)$, then

$$
P\left(\check{\boldsymbol{a}}_{\mathrm{opt}}=\boldsymbol{a}_{\mathcal{I}}\right) \geq P\left(\check{\boldsymbol{a}}=\boldsymbol{a}_{\mathcal{I}}\right)
$$

with $\check{\boldsymbol{a}}$ from any partial integer estimator as defined in (4.2), which meets the criteria (4.4). For a given non-empty index set $\mathcal{I}$ with $|\mathcal{I}|<n$, the optimal integer estimates $\check{\boldsymbol{a}}_{\text {opt }}$ are therefore found by evaluating infinite sums over all integer vectors $\boldsymbol{v} \in \mathbb{Z}^{n}$ with common entries $\boldsymbol{v}_{\mathcal{I}}=\boldsymbol{z}$ that correspond to the index set $\mathcal{I}$. Maximizing the sum over $z$ yields the result. The decision regions 
$S_{z}^{*}$ of the optimal partial integer estimator are given by

$$
\begin{array}{r}
S_{\boldsymbol{z}}^{*}=\left\{\boldsymbol{x} \in \mathbb{R}^{n} \mid \sum_{\boldsymbol{v} \in \mathbb{Z}^{n} \mid \boldsymbol{v}_{\mathcal{I}}=\boldsymbol{z}} \exp \left(-\frac{1}{2}\|\boldsymbol{x}-\boldsymbol{v}\|_{\boldsymbol{Q}_{\hat{\boldsymbol{a}}}}^{2}\right) \geq \sum_{\boldsymbol{v} \in \mathbb{Z}^{n} \mid \boldsymbol{v}_{\mathcal{I}}=\boldsymbol{u}} \exp \left(-\frac{1}{2}\|\boldsymbol{x}-\boldsymbol{v}\|_{\boldsymbol{Q}_{\hat{\boldsymbol{a}}}}^{2}\right), \forall \boldsymbol{u} \in \mathbb{Z}^{|\mathcal{I}|}\right\}, \\
\forall \boldsymbol{z} \in \mathbb{Z}^{|\mathcal{I}|} .
\end{array}
$$

Apart from boundary ties, this estimator leads to a unique $|\mathcal{I}|$ dimensional integer vector for any float solution $\hat{\boldsymbol{a}} \in \mathbb{R}^{n}$. For an arbitrary given integer vector $\boldsymbol{z} \in \mathbb{Z}^{|\mathcal{I}|}$, we have that $\sum_{\boldsymbol{v} \in \mathbb{Z}^{n} \mid \boldsymbol{v}_{\mathcal{I}}=\boldsymbol{z}} \exp \left(-\frac{1}{2}\|\hat{\boldsymbol{a}}-\boldsymbol{v}\|_{\boldsymbol{Q}_{\hat{\boldsymbol{a}}}}^{2}\right)=\sum_{\boldsymbol{w} \in \mathbb{Z}^{n} \mid \boldsymbol{w}_{\mathcal{I}}=\boldsymbol{u}_{\mathcal{I}}+\boldsymbol{z}} \exp \left(-\frac{1}{2}\|\hat{\boldsymbol{a}}+\boldsymbol{u}-\boldsymbol{w}\|_{\boldsymbol{Q}_{\hat{\boldsymbol{a}}}}^{2}\right), \forall \boldsymbol{u} \in \mathbb{Z}^{n}$, since both sums contain the same terms. The maximizing argument of both sums is therefore also identical. Let the maximizing argument $\boldsymbol{z}$, which is the optimal partial integer estimator (4.15) for $\boldsymbol{a}_{\mathcal{I}}$, be denoted by $\check{\boldsymbol{a}}_{\text {opt }} \in \mathbb{Z}^{|\mathcal{I}|}$. With this maximizer, comparing the right sum to (4.15) and interpreting $\hat{\boldsymbol{a}}+\boldsymbol{u}$ as the float solution shows that the optimal estimator for this shifted float solution is $\check{\boldsymbol{a}}_{\text {opt }}+\boldsymbol{u}_{\mathcal{I}}$. The estimator (4.15) is therefore a valid partial integer estimator according to (4.4).

The optimality can be proven as follows. Let the regions $\bar{S}_{\boldsymbol{u}}, \forall \boldsymbol{u} \in \mathbb{Z}^{|\overline{\mathcal{I}}|}$, be arbitrary regions that fulfill the properties of the decision regions $S_{z}$ (4.4) for the index set $\overline{\mathcal{I}}$ instead of $\mathcal{I}$, and let the regions $R_{\boldsymbol{v}}, \forall \boldsymbol{v} \in \mathbb{Z}^{n}$, be defined as $R_{\boldsymbol{v}}=S_{\boldsymbol{v}_{\mathcal{I}}} \cap \bar{S}_{\boldsymbol{v}_{\overline{\mathcal{I}}}}$. This implies that $R_{\boldsymbol{v}}, \forall \boldsymbol{v} \in \mathbb{Z}^{n}$, cover $\mathbb{R}^{n}$ without gaps and overlaps and are integer translated copies of each other for any integer $\in \mathbb{Z}^{n}$. The decision regions $S_{\boldsymbol{z}}$ can then be written as $S_{\boldsymbol{z}}=\bigcup_{\boldsymbol{v} \in \mathbb{Z}^{n} \mid \boldsymbol{v}_{\mathcal{I}}=\boldsymbol{z}} R_{\boldsymbol{v}}$. With $p_{\hat{\boldsymbol{a}}}(\boldsymbol{x})$ the Gaussian pdf of the float solution, the decision regions $S_{z}^{*}(4.17)$ of the optimal estimator for the index set $\mathcal{I}$ can also be written as

$$
S_{\boldsymbol{z}}^{*}=\left\{\boldsymbol{x} \in \mathbb{R}^{n} \mid \sum_{\boldsymbol{v} \in \mathbb{Z}^{n} \mid \boldsymbol{v}_{\mathcal{I}}=\boldsymbol{z}} p_{\hat{\boldsymbol{a}}}(\boldsymbol{x}+\boldsymbol{a}-\boldsymbol{v}) \geq \sum_{\boldsymbol{v} \in \mathbb{Z}^{n} \mid \boldsymbol{v}_{\mathcal{I}}=\boldsymbol{u}} p_{\hat{\boldsymbol{a}}}(\boldsymbol{x}+\boldsymbol{a}-\boldsymbol{v}), \forall \boldsymbol{u} \in \mathbb{Z}^{|\mathcal{I}|}\right\}
$$

Then,

$$
\sum_{\boldsymbol{v} \in \mathbb{Z}^{n} \mid \boldsymbol{v}_{\mathcal{I}}=\boldsymbol{a}_{\mathcal{I}}} p_{\hat{\boldsymbol{a}}}(\boldsymbol{x}+\boldsymbol{a}-\boldsymbol{v}) \geq \sum_{\boldsymbol{u} \in \mathbb{Z}^{|\mathcal{I}|}} \sum_{\boldsymbol{v} \in \mathbb{Z}^{n} \mid \boldsymbol{v}_{\mathcal{I}}=\boldsymbol{u}} p_{\hat{\boldsymbol{a}}}(\boldsymbol{x}+\boldsymbol{a}-\boldsymbol{v}) s_{\boldsymbol{u}}(\boldsymbol{x}), \quad \forall \boldsymbol{x} \in S_{\boldsymbol{a}_{\mathcal{I}}}^{*},
$$

with $s_{\boldsymbol{u}}(\cdot)$ the indicator function of an arbitrary partial integer estimator for subset $\mathcal{I}$ with the decision regions $S_{\boldsymbol{u}}$. We integrate both sides of (4.19) over the subset $R_{\boldsymbol{a}}^{*}=S_{\boldsymbol{a}_{\mathcal{I}}}^{*} \cap \bar{S}_{\boldsymbol{a}_{\overline{\mathcal{I}}}}$ of $S_{\boldsymbol{a}_{\mathcal{I}}}^{*}$, where $\bar{S}_{\boldsymbol{a}_{\overline{\mathcal{I}}}}$ is arbitrary within the above mentioned constraints:

$$
\sum_{\boldsymbol{v} \in \mathbb{Z}^{n} \mid \boldsymbol{v}_{\mathcal{I}}=\boldsymbol{a}_{\mathcal{I}}} \int_{R_{\boldsymbol{a}}^{*}} p_{\hat{\boldsymbol{a}}}(\boldsymbol{x}+\boldsymbol{a}-\boldsymbol{v}) \mathrm{d} \boldsymbol{x} \geq \sum_{\boldsymbol{u} \in \mathbb{Z}^{|\mathcal{I}|}} \sum_{\boldsymbol{v} \in \mathbb{Z}^{n} \mid \boldsymbol{v}_{\mathcal{I}}=\boldsymbol{u}} \int_{R_{\boldsymbol{a}}^{*} \cap S_{\boldsymbol{u}}} p_{\hat{\boldsymbol{a}}}(\boldsymbol{x}+\boldsymbol{a}-\boldsymbol{v}) \mathrm{d} \boldsymbol{x} .
$$

The coordinate transform $\boldsymbol{y}=\boldsymbol{x}+\boldsymbol{a}-\boldsymbol{v}$ leads to (we make use of (iii) from (4.4) for the right side of (4.20), i.e., $S_{\boldsymbol{u}}+\boldsymbol{a}-\boldsymbol{v}=S_{\boldsymbol{u}+\boldsymbol{a}_{\mathcal{I}}-\boldsymbol{v}_{\mathcal{I}}}$ )

$$
\sum_{\boldsymbol{v} \in \mathbb{Z}^{n} \mid \boldsymbol{v}_{\mathcal{I}}=\boldsymbol{a}_{\mathcal{I}}} \int_{R_{2 \boldsymbol{a}-\boldsymbol{v}}^{*}} p_{\hat{\boldsymbol{a}}}(\boldsymbol{y}) \mathrm{d} \boldsymbol{y} \geq \sum_{\boldsymbol{u} \in \mathbb{Z}^{|\mathcal{I}|}} \sum_{\boldsymbol{v} \in \mathbb{Z}^{n} \mid \boldsymbol{v}_{\mathcal{I}}=\boldsymbol{u}} \int_{R_{2 \boldsymbol{a}-\boldsymbol{v}}^{*} \cap S_{\boldsymbol{u}+\boldsymbol{a}_{\mathcal{I}}-\boldsymbol{v}_{\mathcal{I}}}} p_{\hat{\boldsymbol{a}}}(\boldsymbol{y}) \mathrm{d} \boldsymbol{y} .
$$

Since the regions $R_{v}^{*}$ do not overlap, the sum of integrals on the left side of (4.21) can be written as a single integral over the region $\bigcup_{\boldsymbol{v} \in \mathbb{Z}^{n} \mid \boldsymbol{v}_{\mathcal{I}}=\boldsymbol{a}_{\mathcal{I}}} R_{2 \boldsymbol{a}-\boldsymbol{v}}^{*}=\bigcup_{\boldsymbol{v} \in \mathbb{Z}^{n} \mid \boldsymbol{v}_{\mathcal{I}}=a_{\mathcal{I}}} R_{\boldsymbol{v}}^{*}=S_{\boldsymbol{a}_{\mathcal{I}}}^{*}$. On the right side of (4.21) we notice that all terms have in common that $\boldsymbol{v}_{\mathcal{I}}=\boldsymbol{u}$, i.e., $S_{\boldsymbol{u}+\boldsymbol{a}_{\mathcal{I}}-\boldsymbol{v}_{\mathcal{I}}}=S_{\boldsymbol{a}_{\mathcal{I}}}$ and 
constant for all terms of the sums. The only quantity that depends on the argument of the sums are the non-overlapping $R_{2 a-v}^{*}$. We can therefore again replace the sum of integrals with a single integral. Since the two sums simply represent a sum over all $n$ dimensional integer vectors, the region of integration is $\bigcup_{\boldsymbol{v} \in \mathbb{Z}^{n}} R_{2 \boldsymbol{a}-\boldsymbol{v}}^{*} \cap S_{\boldsymbol{a}_{\mathcal{I}}}=\mathbb{R}^{n} \cap S_{\boldsymbol{a}_{\mathcal{I}}}=S_{\boldsymbol{a}_{\mathcal{I}}}$, and we finally have

$$
\int_{S_{\boldsymbol{a}_{\mathcal{I}}^{*}}^{*}} p_{\hat{\boldsymbol{a}}}(\boldsymbol{y}) \mathrm{d} \boldsymbol{y} \geq \int_{S_{\boldsymbol{a}_{\mathcal{I}}}} p_{\hat{\boldsymbol{a}}}(\boldsymbol{y}) \mathrm{d} \boldsymbol{y} .
$$

Comparing (4.22) to (4.5) shows that the left and right side of (4.22) are the probabilities of correctly resolving $\boldsymbol{a}_{\mathcal{I}}$ for the two estimators defined by the decision regions $S_{\boldsymbol{a}_{\mathcal{I}}}^{*}$ and $S_{\boldsymbol{a}_{\mathcal{I}}}$, which concludes the proof.

If $\mathcal{I}$ corresponds to the full set of integers, the optimal partial integer estimator (4.15) reduces to integer least-squares, since the sum then only contains one term. The success rate of the optimal partial integer estimator cannot be easily computed, but due to the optimality the success rate of any other partial integer estimator serves as a lower bound, e.g., the easily computable success rate of partial integer bootstrapping from Section 4.3.1. The decision regions $S_{z}^{*}$ of the optimal partial integer estimator are illustrated in Figure 4.1 and 4.2 for two different two dimensional examples for $\mathcal{I}=\{1\}$ and $\mathcal{I}=\{2\}$. It is noted that if the covariance matrix of the float estimate $\hat{\boldsymbol{a}}$ can be factorized as $\boldsymbol{Q}_{\hat{\boldsymbol{a}}}=\sigma^{2} \boldsymbol{G}$, then the computation of the integer least-squares solution does not depend on $\sigma$, i.e., an overall increased noise level has no impact on the integer estimates $\check{\boldsymbol{a}}_{\text {parILS }}$ and $\check{\boldsymbol{a}}_{\text {fullILS. }}$. The optimal integer estimator does, however, depend on the scaling of the covariance matrix, so that the decision regions are different for different values of $\sigma$.

\subsubsection{Computation of the Optimal Estimator}

In this section we discuss the problem of how to implement the optimal partial integer estimator (4.15) for any index set $\mathcal{I}$ that is not the full set. For $|\mathcal{I}|=n$, (4.15) reduces to a standard integer least-squares problem, which is discussed in Section 3.1.4. An equivalent formulation of the optimization problem (4.15) is given by

$$
\check{\boldsymbol{a}}_{\mathrm{opt}}=\underset{\boldsymbol{z} \in \mathbb{Z}^{|\mathcal{I}|}}{\operatorname{argmin}} \sum_{\boldsymbol{v} \in \mathbb{Z}^{n} \mid \boldsymbol{v}_{\mathcal{I}} \neq \boldsymbol{z}} \exp \left(-\frac{1}{2}\|\hat{\boldsymbol{a}}-\boldsymbol{v}\|_{\boldsymbol{Q}_{\hat{\boldsymbol{a}}}}^{2}\right)=\underset{\boldsymbol{z} \in \mathbb{Z}^{|\mathcal{I}|}}{\operatorname{argmin}} C(\boldsymbol{z}),
$$

with $C(\boldsymbol{z})$ the cost function as defined in (4.23). The minimization of this cost function over the set $\mathbb{Z}^{|\mathcal{I}|}$ can be solved via search (and shrink) as follows. The cost function $C(\boldsymbol{z})$ is initialized with, e.g., $\boldsymbol{z}=\check{\boldsymbol{a}}_{\mathrm{ILS}, \mathcal{I}}$ as $C\left(\check{\boldsymbol{a}}_{\mathrm{ILS}, \mathcal{I}}\right)=d$, where $\check{\boldsymbol{a}}_{\mathrm{ILS}}$ is the $n$ dimensional integer least-squares solution. The goal is now to find all integer candidates $\boldsymbol{z} \in \mathbb{Z}^{|\mathcal{I}|}$ with $C(\boldsymbol{z}) \leq d$. The integer candidate with the minimum value of the cost function $C(\cdot)$ is the optimizer of (4.23). This search strategy can be combined with a shrinking of the search space, i.e., whenever a valid integer candidate $z$ is found, the value of $d$ is reduced to $C(\boldsymbol{z})$. The search for integer candidates $\boldsymbol{z} \in \mathbb{Z}|\mathcal{I}|$ can be formulated as a tree-search problem, as the cost function can be written as

$$
C(\boldsymbol{z})=\sum_{i=1}^{|\mathcal{I}|} c_{i}\left(z_{1}, \ldots, z_{i}\right)
$$

where $c_{i}\left(z_{1}, \ldots, z_{i}\right)$ are non-negative additive increments that only depend on all entries of $\boldsymbol{z}$ up to level $i$. They are given by

$$
c_{i}\left(z_{1}, \ldots, z_{i}\right)=\sum_{\substack{\left.v_{\mathcal{V}_{\mathcal{I}} \neq Z_{i}}{ }^{n}\right|_{\mathcal{V}_{\mathcal{I}_{1}}=z_{1}, \ldots, v_{\mathcal{I}_{i-1}}=z_{i-1}}\\}} \exp \left(-\frac{1}{2}\|\hat{\boldsymbol{a}}-\boldsymbol{v}\|_{\boldsymbol{Q}_{\hat{\boldsymbol{a}}}}^{2}\right),
$$


with $\mathcal{I}_{i}$ the $i$ th element of the index set. That is, the sum in (4.23) for computing $C(\boldsymbol{z})$ can be built up by adding new terms in each level. The possible values at level $i$ follow from $c_{i}\left(z_{1}, \ldots, z_{i}\right) \leq$ $d-\sum_{j=1}^{i-1} c_{j}\left(z_{1}, \ldots, z_{j}\right)$.

Since the evaluation of $C(\boldsymbol{z})$ in (4.23) comprises a sum over infinitely many integer vectors $\boldsymbol{v}$, it cannot be computed exactly but has to be approximated, i.e., the integer vectors $\boldsymbol{v}$ have to be chosen from a finite set instead of $\mathbb{Z}^{n}$. This finite set is defined such that all integers, whose contribution to the sum is too small, are omitted. This results in the set $\Theta_{\hat{a}}^{\lambda}$ of integers, which lie within an ellipsoidal region centered around $\hat{\boldsymbol{a}}$, i.e.,

$$
\Theta_{\boldsymbol{x}}^{\lambda}=\left\{\boldsymbol{v} \in \mathbb{Z}^{n} \mid\|\boldsymbol{x}-\boldsymbol{v}\|_{\boldsymbol{Q}_{\hat{a}}}^{2}<\lambda^{2}\right\}
$$

The larger the value of the size parameter $\lambda$ of $\Theta_{\hat{a}}^{\lambda}$ is chosen, the closer the approximation will be to the exact value of $C(\boldsymbol{z})$. The same problem arises in the computation of the best integer equivariant estimator, where a good choice for $\lambda$ was found from the condition $P\left(\|\hat{\boldsymbol{a}}-\boldsymbol{a}\|_{\boldsymbol{Q}_{\hat{\boldsymbol{a}}}}^{2}<\lambda^{2}\right)=1-\alpha$ with a small value of $\alpha$ (Teunissen 2005d). Since $\|\hat{\boldsymbol{a}}-\boldsymbol{a}\|_{\boldsymbol{Q}_{\hat{\boldsymbol{a}}}}^{2}$ follows a central $\chi^{2}$ distribution with $n$ degrees of freedom, the value of $\lambda$ can be determined.

The evaluation of (4.25) includes a search for integer candidates in $\Theta_{\hat{a}}^{\lambda}$ in each level $i=$ $1, \ldots,|\mathcal{I}|$. In order to save computational complexity, one should first search for all integers $\boldsymbol{v} \in \Theta_{\hat{\boldsymbol{a}}}^{\lambda}$ and store them together with $\exp \left(-\frac{1}{2}\|\hat{\boldsymbol{a}}-\boldsymbol{v}\|_{\boldsymbol{Q}_{\hat{a}}}^{2}\right)$. Irrespective of the chosen parameterization via $Z$ in (2.82), this first search should be carried out in a decorrelated space, which makes the search more efficient. The search for the optimal solution $\check{\boldsymbol{a}}_{\text {opt }} \in \mathbb{Z}^{|\mathcal{I}|}$ is then performed by simply selecting candidates from that list when evaluating (4.25).

\subsubsection{Optimal vs. Suboptimal Partial Integer Estimation}

In Section 4.3.4 it is explained, how the optimal partial integer estimator as derived in Section 4.3.3 can be efficiently implemented. One might, however, be interested in using one of the simpler partial integer estimators (4.11) or (4.12), which only require an integer least-squares solution, such as it is provided by, e.g., the LAMBDA method. If the sum in the computation of the optimal estimator (4.15) is simply approximated by its maximum term, (4.15) becomes equivalent to selecting entries of the full integer least-squares solution, i.e., to (4.13). Furthermore, we can analyze (4.15) for the case that $\hat{\boldsymbol{a}}$ is of very high precision. A constant factor does not change the result of (4.15), thus

$$
\check{\boldsymbol{a}}_{\mathrm{opt}}=\underset{\boldsymbol{z} \in \mathbb{Z}^{|\mathcal{I}|}}{\operatorname{argmax}} \sum_{\boldsymbol{v} \in \mathbb{Z}^{n} \mid \boldsymbol{v}_{\mathcal{I}}=\boldsymbol{z}} \frac{\exp \left(-\frac{1}{2}\|\hat{\boldsymbol{a}}-\boldsymbol{v}\|_{\boldsymbol{Q}_{\hat{a}}}^{2}\right)}{\sum_{\boldsymbol{u} \in \mathbb{Z}^{n}} \exp \left(-\frac{1}{2}\|\hat{\boldsymbol{a}}-\boldsymbol{u}\|_{\boldsymbol{Q}_{\hat{\boldsymbol{a}}}}^{2}\right)}=\underset{\boldsymbol{z} \in \mathbb{Z}^{|\mathcal{I}|}}{\operatorname{argmax}} \sum_{\boldsymbol{v} \in \mathbb{Z}^{n} \mid \boldsymbol{v}_{\mathcal{I}}=\boldsymbol{z}} w_{\boldsymbol{v}}(\hat{\boldsymbol{a}}) .
$$

With $w_{\boldsymbol{v}}(\hat{\boldsymbol{a}})=\frac{1}{1+\sum_{\boldsymbol{u} \in \mathbb{Z}^{n} \mid \boldsymbol{u} \neq \boldsymbol{v}} \exp \left(-\frac{1}{2 \sigma^{2}}\left(\|\hat{\boldsymbol{a}}-\boldsymbol{u}\|_{G^{-}}^{2}-\|\hat{\boldsymbol{a}}-\boldsymbol{v}\|_{G}^{2}\right)\right)}$, where the factorization $\boldsymbol{Q}_{\hat{\boldsymbol{a}}}=\sigma^{2} \boldsymbol{G}$ is used, it follows that $\lim _{\sigma \rightarrow 0} w_{\boldsymbol{v}}(\hat{\boldsymbol{a}})=1$, if $\|\hat{\boldsymbol{a}}-\boldsymbol{v}\|_{\boldsymbol{G}}^{2} \leq\|\hat{\boldsymbol{a}}-\boldsymbol{u}\|_{\boldsymbol{G}}^{2}, \forall \boldsymbol{u} \in \mathbb{Z}^{n}$, and $\lim _{\sigma \rightarrow 0} w_{\boldsymbol{v}}(\hat{\boldsymbol{a}})=0$, else. Since the integer least-squares solution does not depend on $\sigma$ for the above factorization, this means that $w_{\check{\boldsymbol{a}}_{\mathrm{ILS}}}(\hat{\boldsymbol{a}})=1$, with $\check{\boldsymbol{a}}_{\mathrm{ILS}}$ the $n$ dimensional integer least-squares solution, and $w_{\boldsymbol{v}}(\hat{\boldsymbol{a}})=0, \forall \boldsymbol{v} \in \mathbb{Z}^{n} \backslash \check{\boldsymbol{a}}_{\mathrm{ILS}}$. Accordingly, the sum in (4.27) will reach its maximum, if the full integer least-squares solution $\check{\boldsymbol{a}}_{\mathrm{ILS}}$ is contained, and the optimal partial integer mapping becomes identical to selecting entries of the full integer least-squares solution (4.13).

These arguments in favor of selecting entries of the full integer least-squares solution over partial integer least-squares are verified in a numerical study with simulated single epoch dual frequency L1+L2 single baseline GPS positioning examples. The satellite constellation is used 


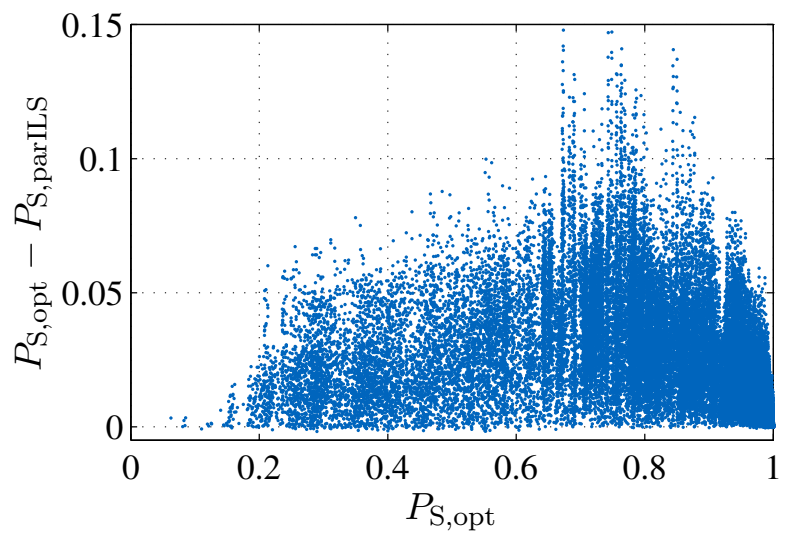

(a) Partial integer least-squares

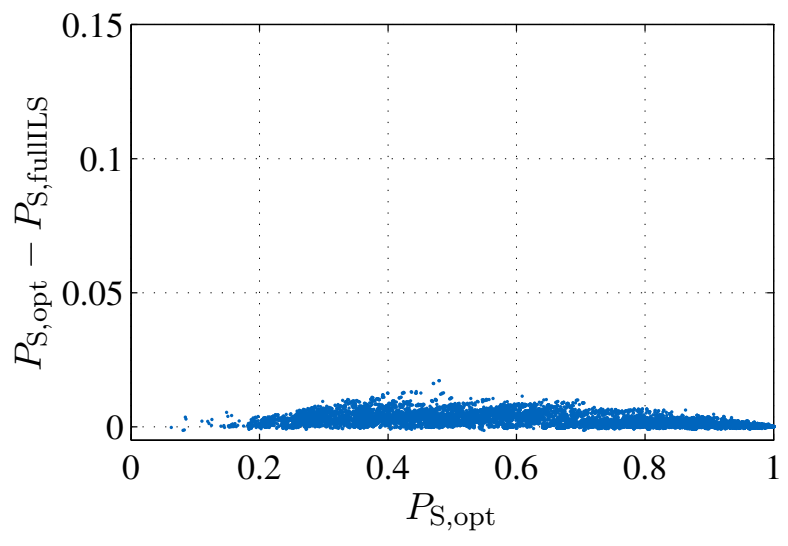

(b) Full integer least-squares + selection

Figure 4.3: Difference between success rates of the suboptimal partial integer estimators and the optimal partial integer estimator; each figure shows 68,000 data points.

as seen during GPS week 1,815 with an elevation cutoff angle of $10^{\circ}$. We consider 145 different epochs in the area of Munich, Germany. The standard deviation of the undifferenced measurements in zenith direction is assumed as $25 \mathrm{~cm}$ for code and $3 \mathrm{~mm}$ for phase observations, to which the elevation dependent exponential weighting as explained in Section 2.3 is applied. The ionosphere weighted model with standard deviations of $0-3 \mathrm{~cm}$ for the between receiver differential ionospheric delays in zenith direction is used. Differential tropospheric delays are assumed absent.

Figure 4.3 shows the difference between the success rates of the optimal scheme and partial ILS/full ILS + selection, which are computed via Monte Carlo integration. For each positioning case, the success rates are evaluated for all subsets $\mathcal{I}$ except $\mathcal{I}=\{1, \ldots, n\}$, which lead to a precision of

$$
\left[\begin{array}{c}
\sigma_{\mathrm{E}} \\
\sigma_{\mathrm{N}} \\
\sigma_{\mathrm{U}}
\end{array}\right] \leq\left[\begin{array}{l}
2 \mathrm{~cm} \\
2 \mathrm{~cm} \\
6 \mathrm{~cm}
\end{array}\right],
$$

where $\sigma_{\mathrm{E}}, \sigma_{\mathrm{N}}$, and $\sigma_{\mathrm{U}}$ are the standard deviations of the coordinate estimates in the local east, north, and up frame that follow from the conditional covariance matrix $\boldsymbol{Q}_{\hat{\boldsymbol{b}} \mid \hat{\boldsymbol{a}}_{\mathcal{T}}}$. That is, each data point in the figures corresponds to a different combination of measurement epoch, ionospheric uncertainty, and ambiguity subset. All partial integer fixing schemes are applied in the LAMBDA decorrelated space (Teunissen 1995b). While partial integer least-squares loses up to $15 \%$ in terms of the success rate compared to the optimal fixing scheme, full integer least-squares + selection performs close to optimal for all examples.

Table 4.1: Average computation times of the search algorithms for partial integer least-squares, full integer least-squares + selection, and the optimal estimator.

\begin{tabular}{llll}
\hline & Partial ILS & Full ILS + selection & Optimal \\
\hline Search for integer candidate(s) & $0.056 \mathrm{~ms}$ & $0.059 \mathrm{~ms}$ & $0.446 \mathrm{~ms}$ \\
Search for integer solution & - & - & $1.970 \mathrm{~ms}$ \\
Total search time & $0.056 \mathrm{~ms}$ & $0.059 \mathrm{~ms}$ & $2.416 \mathrm{~ms}$ \\
\hline
\end{tabular}

In order for the optimal partial integer estimator to be used in real time GNSS applications, its computation time must be sufficiently small. Table 4.1 shows the average computation times of 
the search algorithms required to find the integer estimates for the above numerical examples. The timing was done using Matlab implementations on a standard desktop computer. Partial integer least-squares and full integer least-squares + selection only require the search for a closest integer candidate, which is slightly faster for partial integer least-squares due to the smaller dimension of the search problem. For the optimal scheme, all integer candidates within $\Theta_{\hat{a}}^{\lambda}(4.26)$ have to be found instead of only the best candidate, which causes a longer time for the candidate search. A value of $\alpha=0.001$ is used to determine the size parameter $\lambda$ of $\Theta_{\hat{a}}^{\lambda}$. As explained in Section 4.3.4, a second search is required to determine the integer solution $\check{\boldsymbol{a}}_{\text {opt }}$ from these candidates. Although the average total search time of the optimal partial fixing scheme is clearly larger than the ones of the two suboptimal estimators, it is still well within the real time requirements for GNSS applications. It is noted that these timing results of course strongly depend on the employed hardware and implementation and should therefore only be seen as a rough indicator for the order of magnitude that can be expected for the computation time. Additional complexity resides in the LAMBDA decorrelation transformation that was applied before the search algorithms, which is identical for all three methods.

\subsection{Selecting the Integer Transformation and Subset}

So far, the problem of how to estimate a given subset $\mathcal{I}$ of the integer parameters for a given parameterization via $Z$ was discussed. Depending on the problem at hand, it might be a priori clear which subset of which parameter combinations has to be estimated. In GNSS positioning applications the goal is to obtain a good positioning quality. The choice of the integer transformation and ambiguity subset per se is not important, as long as this goal is reached. Possible integer transformations and criteria that can be used to select the subset $\mathcal{I}$ of integer ambiguities as well as selection strategies are discussed in the following.

\subsubsection{Integer Transformations for Partial Ambiguity Resolution}

With the integer transformation matrix $\boldsymbol{Z} \in \mathbb{Z}^{n \times n}$, the subset $\mathcal{I}$ of the integer parameter vector $\boldsymbol{Z} \boldsymbol{a}$ is resolved, i.e., the rows of the integer transformation define the estimable linear combinations of the parameters. Ideally, the transformation matrix $Z$ and the index set $\mathcal{I}$ should be chosen jointly in GNSS applications so as to result in an as good as possible positioning quality. Since there is, however, an infinite amount of possibilities for $Z$ with a large number of possible index sets $\mathcal{I}$ for each choice, this is hardly feasible in practice. A reasonable approach is thus to first choose $Z$ and then to find a subset according to certain criteria as discussed in Section 4.4.2.

A trivial choice for the integer transformation is $Z=\boldsymbol{I}_{n}$, which simply means that a subset of the original double difference ambiguities is resolved. As it is shown in Teunissen (1997b), the precision of the original float ambiguities is very poor for short baselines, but they are also highly correlated, meaning that the confidence ellipse of the float solution $\hat{\boldsymbol{a}}$ is very much elongated. It is intuitively clear that it is difficult to resolve a true subset of the integer parameters in such a situation, which can be demonstrated as follows. When evaluating the sum for computing the optimal partial integer estimator (4.15) for a given subset $\mathcal{I}$, this implies that the contribution of the terms corresponding to integers other than the closest integer $\check{\boldsymbol{a}}_{\mathrm{ILS}}$ is rather small, since the integer vectors with identical entries for the given subset are generally far away from the float solution in the metric of $\boldsymbol{Q}_{\hat{\boldsymbol{a}}}$, and $\check{\boldsymbol{a}}_{\text {opt }}$ is then mainly determined based on selecting entries of $\check{\boldsymbol{a}}_{\text {ILS }}$. Further, due to the high correlation, the most likely candidates for the integer least-squares solution $\check{\boldsymbol{a}}_{\mathrm{ILS}}$ 
are-after the true integer vector $\boldsymbol{a}$-generally not the ones for which many entries are identical with $a$. For the high correlation case, one can therefore generally not expect too large gains in the success rate when resolving a subset of the integer parameters as compared to the success rate of conventional full integer least-squares.

Following this argumentation, decorrelation transformations as introduced in Section 3.1.5 are very useful for resolving a subset of the integer parameters. This is also intuitively clear, since when the components of the float solution $\hat{\boldsymbol{a}}$ are largely decorrelated, it should be much easier to resolve only some of them as integers. It is, however, noted that although such decorrelation transformations may increase the probability of correctly resolving a certain subset $\mathcal{I}$ of the integer parameters, they also change the influence on the precision of the real valued parameters like the receiver coordinates.

A different strategy used in GNSS applications is to formulate $Z$ such that some of the rows correspond to so-called widelane ambiguities and to subsequently resolve only those (Cao et al. 2007). The concept of widelaning is motivated by forming linear combinations of the carrier-phase measurement on multiple frequencies for the same double difference link. By properly choosing the coefficients, the common geometric range is preserved and the combination can be interpreted as a single carrier-phase measurement with the integer ambiguity $N_{r q}^{s k}=\sum_{f=1}^{F} k_{f} N_{r q, f}^{s k}$, where $k_{f} \in \mathbb{Z}, f=1, \ldots, F$. The artificial wavelength of this combination is given by

$$
\lambda=\frac{1}{\sum_{f=1}^{F} \frac{k_{f}}{\lambda_{f}}} .
$$

The coefficients used for computing the combination are often chosen such that the wavelength $\lambda$ is increased compared to all $\lambda_{f}$ in order to facilitate resolving the ambiguity $N_{r q}^{s k}$, while the noise and ionospheric delay are not too large, see, e.g., Hatch (1982), Wübbena (1989), Euler and Goad (1991), Cocard and Geiger (1992), Teunissen (1997d), Bock (1998), Henkel and Günther (2007), and Cocard et al. (2008). The gain of the positioning precision that is obtained when resolving only the conventional GPS widelane ambiguities with $k_{1}=1$ and $k_{2}=-1$ for L1 and L2 is shown in Odijk et al. (2014a) to be almost negligible. A limitation of widelane, narrowlane, and all other multi-frequency linear combinations is that they only allow for combinations of ambiguities $N_{r q, f}^{s k}$ that correspond to the same pair of satellites $k$ and $s$ and the same receivers $q$ and $r$. The choice of $\boldsymbol{Z}$ when only such ambiguity combinations are used is therefore quite restricted. The topic of finding optimized multi-frequency combinations is not further considered in this dissertation.

\subsubsection{Subset Selection Criteria}

In GNSS applications, there are essentially two criteria that the user is interested in when selecting the subset $\mathcal{I}$ of integer parameters to be resolved, which are connected to each other: The precision of the estimate $\check{\boldsymbol{b}}$ that he can expect after resolving $\boldsymbol{a}_{\mathcal{I}}$, and the reliability of the integer estimate $\check{\boldsymbol{a}}$.

As it was demonstrated in Section 4.2, the estimate $\breve{b}$ is Gaussian distributed with mean value $\boldsymbol{b}$ and the conditional covariance matrix $\boldsymbol{Q}_{\hat{b} \mid \hat{\boldsymbol{a}}_{\mathcal{I}}}$ for the given subset $\mathcal{I}$, if the integer estimate $\check{\boldsymbol{a}}$ is assumed to be correct and deterministic as $\check{\boldsymbol{a}}=\boldsymbol{a}_{\mathcal{I}}$. The probability of an incorrect ambiguity estimate $\check{\boldsymbol{a}}$ directly tells us how much confidence we can put in the presented statistics of $\check{\boldsymbol{b}}$. If this failure rate is sufficiently small, the user can employ $\boldsymbol{Q}_{\hat{\boldsymbol{b}} \mid \hat{\boldsymbol{a}}_{\mathcal{I}}}$ from (4.9) to evaluate which precision he can expect from the estimate $\breve{b}$ after the algorithm that he chose determined the transformation $Z$ and the index set $\mathcal{I}$. If the user application requires a certain minimum positioning precision, the 
conditional covariance matrix $\boldsymbol{Q}_{\hat{b} \mid \hat{\boldsymbol{A}}_{\mathcal{I}}}$ can be used to decide, whether a specific subset $\mathcal{I}$ is useful for the application or not. Since

$$
\boldsymbol{Q}_{\hat{b} \mid \hat{a}_{\mathcal{I}}} \preccurlyeq \boldsymbol{Q}_{\hat{b} \mid \hat{a}_{\mathcal{J}}}, \quad \forall \mathcal{J} \subseteq \mathcal{I}
$$

where $\preccurlyeq$ is defined in terms of positive definiteness, we know that once we have found an index set $\mathcal{I}$ that does not lead to the required precision of $\check{b}$, all subsets of $\mathcal{I}$ cannot reach that precision as well and do not have to be considered.

The reliability can be measured with the probability $P\left(\check{\boldsymbol{a}}=\boldsymbol{a}_{\mathcal{I}}\right)$ of a correct integer estimate $\check{\boldsymbol{a}}$, cf. (4.5). As mentioned above, this success rate is important, since the precision as given by the conditional covariance matrix is only valid given that $\check{\boldsymbol{a}}=\boldsymbol{a}_{\mathcal{I}}$, otherwise $\check{\boldsymbol{b}}$ can have large errors. The subset $\mathcal{I}$ should therefore be selected such that the corresponding failure rate of the employed partial integer estimator does not exceed a user-defined maximum tolerable value. For full integer least-squares + selection and for the optimal estimator, we can make a statement similar to (4.30) for the failure rate:

$$
\begin{gathered}
P\left(\check{\boldsymbol{a}}_{\text {fullILS }} \neq \boldsymbol{a}_{\mathcal{I}}\right) \geq P\left(\check{\boldsymbol{a}}_{\text {fullILS }} \neq \boldsymbol{a}_{\mathcal{J}}\right), \quad \forall \mathcal{J} \subseteq \mathcal{I}, \\
P\left(\check{\boldsymbol{a}}_{\text {opt }} \neq \boldsymbol{a}_{\mathcal{I}}\right) \geq P\left(\check{\boldsymbol{a}}_{\text {opt }} \neq \boldsymbol{a}_{\mathcal{J}}\right), \quad \forall \mathcal{J} \subseteq \mathcal{I} .
\end{gathered}
$$

For full integer least-squares + selection, (4.31) can easily be verified using the definition of the decision regions in (4.14), which are the union of pull-in regions of $n$ dimensional integer leastsquares. The region that leads to a failure for any index set $\mathcal{J} \subseteq \mathcal{I}$ is contained in the region that leads to an error for the index set $\mathcal{I}$. Further, let $S_{z}^{*}$ denote the decision regions of the optimal partial integer estimator for $\mathcal{I}$. For any $\mathcal{J} \subseteq \mathcal{I}$, the decision regions could for instance be chosen as the union of all regions $S_{\boldsymbol{z}}^{*}$ that correspond to different integer values for the elements that have been removed from the index set, and to identical integer values for the elements that are still in the index set. The resulting estimator would then already fulfill (4.31) and is not even optimal, which shows that (4.31) is valid. Consequently, we know that once we have found an index set $\mathcal{I}$ that exceeds the failure rate constraint, all index sets that include $\mathcal{I}$ cannot fulfill the failure rate constraint either and do not have to be considered. It is noted that the corresponding statement for partial integer least-squares is not valid, i.e., $P\left(\check{\boldsymbol{a}}_{\text {parILS }} \neq \boldsymbol{a}_{\mathcal{I}}\right)$ can also be smaller than $P\left(\check{\boldsymbol{a}}_{\text {parILS }} \neq \boldsymbol{a}_{\mathcal{J}}\right)$ for $\mathcal{J} \subseteq \mathcal{I}$.

We can now evaluate the precision and failure rates for different choices of the subset $\mathcal{I}$ and based on the result decide upon which subset to finally choose when applying the partial integer estimator to the float solution $\hat{\boldsymbol{a}}$. This is the principle of model-driven reliable partial ambiguity resolution, since the choice of the subset $\mathcal{I}$ only depends on the description of the system model. Compared to model-driven full ambiguity resolution as discussed in Section 3.1.1, the decision of whether or not to resolve the ambiguities is extended by which subset to resolve.

One could also think of other criteria for selecting the subset $\mathcal{I}$ like the elevation angles of the satellites (Mowlam 2004) or the duration of satellite tracking. Both these criteria require a parameterization of the ambiguities with $\boldsymbol{Z}=\boldsymbol{I}_{n}$, so that the double difference ambiguities corresponding to a specific satellite-for a given reference satellite-can be removed. Since the properties of the float solution $\hat{\boldsymbol{a}}$ are fully captured by the statistical description via $\boldsymbol{Q}_{\hat{\boldsymbol{a}}}$, it is, however, rather useless to consider such criteria.

\subsubsection{Subset Selection Strategies}

With the two above selection criteria one would ideally have to evaluate the failure rate and precision of all possible ambiguity subsets and choose the one that is best for the user application, 


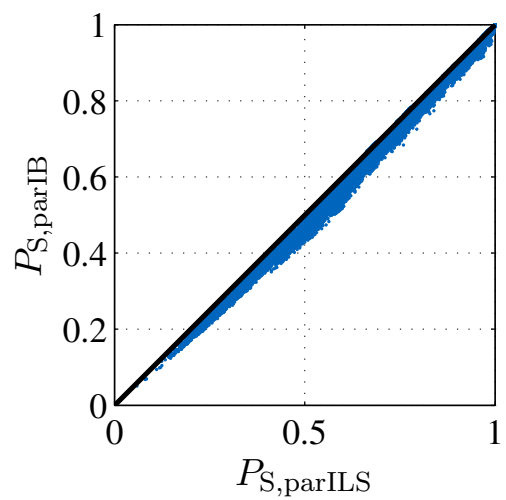

(a) Partial integer least-squares

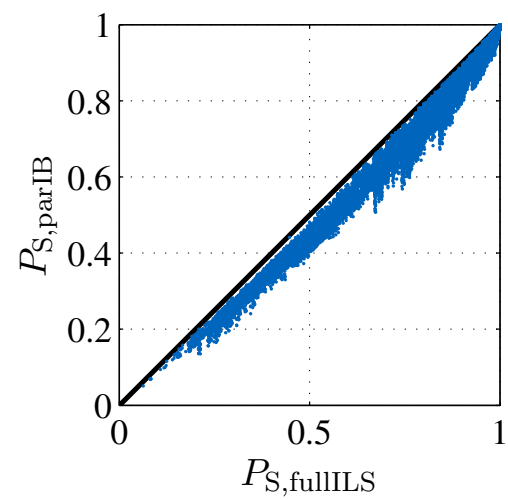

(b) Full integer least-squares + selection

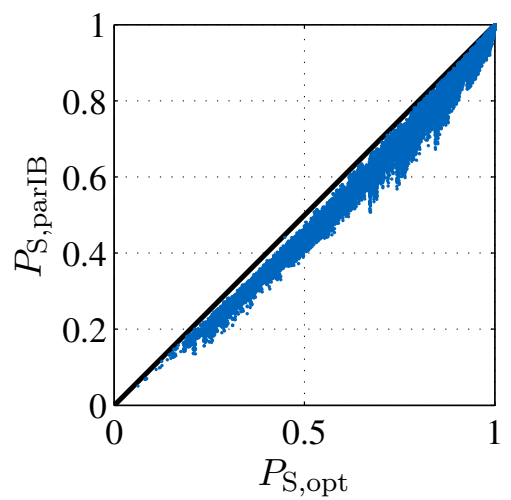

(c) Optimal partial integer estimation

Figure 4.4: Success rates of partial integer bootstrapping versus the actual success rates of partial integer least-squares (left), full integer least-squares + selection (center), and the optimal scheme (right); each figure shows 68,000 data points.

where best has to be properly defined. In the following we only consider strategies with a maximum failure rate constraint, i.e., we may choose any of the subsets that lead to a sufficiently small probability of an incorrect integer estimate when applying a partial integer estimator, which makes the selection problem somewhat simpler. Still, one would ideally have to find all subsets for which the failure rate constraint is met and choose the one that leads to the most precise estimates as defined by $\boldsymbol{Q}_{\hat{\boldsymbol{b}} \mid \hat{\boldsymbol{a}}_{\mathcal{I}}}$. Since the failure rates of both the optimal partial integer estimator and the two presented suboptimal schemes cannot be computed in closed form, they have to be approximated, e.g., via Monte Carlo integration, or bounded. As it was discussed above, an upper bound for the failure rate of partial integer least-squares (4.11) and the optimal scheme (4.15) is given by the failure rate of partial integer bootstrapping, which can be computed using (3.12) with $\boldsymbol{Q}_{\hat{\boldsymbol{a}}_{\mathcal{I}}}$ instead of $\boldsymbol{Q}_{\hat{\boldsymbol{a}}}$. If the difference between the easy-to-compute integer bootstrapping failure rates and the failure rates of the presented partial integer estimators is small, we can use the integer bootstrapping failure rates to decide whether or not a certain subset $\mathcal{I}$ of integers can be reliably resolved, without being too conservative.

Figure 4.4 shows the analytic success rates $P_{\mathrm{S} \text {,parBS }}$ of partial integer bootstrapping against the success rates $P_{\mathrm{S} \text {,parILS }}$ of partial integer least-squares, cf. (4.11), $P_{\mathrm{S} \text {,fullILS }}$ of full integer leastsquares + selection, cf. (4.13), and $P_{\mathrm{S} \text {,opt }}$ of optimal partial integer fixing, cf. (4.15), which are computed via Monte Carlo integration. The simulation settings are the same as in Section 4.3.5. We can see that the success rates of partial integer bootstrapping are a tighter lower bound for partial integer least-squares than for the optimal estimator, which again shows that $P_{\mathrm{S} \text {,opt }}$ is in general noticeably larger than $P_{\mathrm{S} \text {,parILS }}$. Also, the integer bootstrapping success rates seem to work well as a lower bound for full integer least-squares + selection, although they cannot be guaranteed to be a lower bound.

The number of possible subsets $\mathcal{I}$ might become very large, especially if multiple GNSS and/or frequencies are used. It is therefore reasonable to think about simpler strategies for selecting $\mathcal{I}$, which only require the evaluation of the failure rates of a few subsets. One possibility for selecting the subset is given by $\mathcal{I}=\left\{n_{\mathrm{IB}}, \ldots, n\right\}$, with

$$
n_{\mathrm{IB}}=\underset{j \in\{1, \ldots, n\}}{\operatorname{argmax}} P_{\mathrm{F}, \mathrm{IB}, j} \quad \text { s.t. } \quad P_{\mathrm{F}, \mathrm{IB}, j} \leq \gamma,
$$


where $\gamma$ is the maximum tolerable failure rate. The partial integer bootstrapping failure rate $P_{\mathrm{F}, \mathrm{IB}, j}$ can be computed in closed form as

$$
P_{\mathrm{F}, \mathrm{IB}, j}=1-\prod_{i=j}^{n}\left(2 \Phi\left(\frac{1}{2 \sigma_{\hat{a}_{i \mid I}}}\right)-1\right),
$$

where $\sigma_{\hat{a}_{i \mid I}}$ are the conditional standard deviations as given by $\boldsymbol{Q}_{\hat{\boldsymbol{a}}}$ and $I=\{i+1, \ldots, n\}$, cf. Section 3.1.3. This is identical to computing the integer bootstrapping failure rate based on $\boldsymbol{Q}_{\hat{\boldsymbol{a}}_{\mathcal{I}}}$ for $\mathcal{I}=\{j, \ldots, n\}$, with $j \in\{1, \ldots, n\}$. The index set $\mathcal{I}$ is determined by iteratively adding elements until the failure rate becomes too large. This idea of using a truncated version of the sequential integer bootstrapping method for reliable partial GNSS ambiguity resolution is introduced in Teunissen et al. (1999) and used in, e.g., Khanafseh and Pervan (2010), Verhagen et al. (2011), and Odijk et al. (2014a).

A different strategy is proposed in Nardo et al. (2016). The idea is to start with the full set of integer parameters $\mathcal{I}=\{1, \ldots, n\}$ and to iteratively remove the index corresponding to the least precise element of the float solution in each step until the failure rate drops below the maximum tolerable value. 


\section{Generalized Integer Aperture Estimation}

The class of integer aperture estimators as introduced in Section 3.2 is generalized so as to be capable of resolving a subset $\mathcal{I}$ of the integer parameters $a$. The index set $\mathcal{I}$ of such a generalized integer aperture estimator can depend both on the system model and the realization of the float solution $\hat{\boldsymbol{a}}$, which is a much more flexible approach than the one of partial integer estimation in Chapter 4. The definition of generalized integer aperture estimators is given in Section 5.1 and the quality of the estimates is analyzed in Section 5.2. Then, some specific examples of generalized integer aperture estimators are discussed, where we distinguish between estimators with a single non-empty integer subset in Section 5.3 and multiple non-empty integer subsets in Section 5.4. A user friendly method to determine critical values required for acceptance test based (generalized) integer aperture estimators by means of a simple functional approximation is introduced in Section 5.5. Within this chapter, $\hat{\boldsymbol{a}}$ and $\check{\boldsymbol{a}}$ are used instead of $\hat{\boldsymbol{a}}^{\prime}$ and $\check{\boldsymbol{a}}^{\prime}$ for the estimates after the transformation with $Z$.

\subsection{The Class of Generalized Integer Aperture Estimators}

Like for integer aperture estimators, also the index set $\mathcal{I}$ of a generalized integer aperture estimator representing the elements of the integer parameter vector $\boldsymbol{a}$ to be resolved depends on the realization of the float estimate $\hat{\boldsymbol{a}}$ and is thus a random variable assuming discrete values. The values that can be assumed by $\mathcal{I}$, however, are no longer limited to only the full set $\{1, \ldots, n\}$ and the empty set, but can generally be any of the $2^{n}$ possibilities. Let the set of all possible realizations of $\mathcal{I}$ be given by $\mathfrak{I}$, with $1 \leq|\mathfrak{I}| \leq 2^{n}$. Note that $\mathfrak{I}$ has to contain at least one element, even if this is only the empty set $\emptyset$. Which elements are specifically contained in $\mathfrak{I}$ is defined by the choice of the estimator. Every possible realization $\mathcal{J} \in \mathfrak{I}$ of the index set $\mathcal{I}$ is associated to the respective point set $\Omega_{\mathcal{J}} \subseteq \mathbb{R}^{n}$, which describes the region of $\hat{\boldsymbol{a}}$ for which the subset $\mathcal{J}$ of the integer parameters is resolved. These point sets can thus be interpreted as selective aperture regions. Accordingly, the index set $\mathcal{I}$ is given by

$$
\mathcal{I}=\bigcup_{\mathcal{J} \in \mathfrak{I}} \omega_{\mathcal{J}}(\hat{\boldsymbol{a}}) \cap \mathcal{J}
$$

with the indicator function for sets

$$
\omega_{\mathcal{J}}(\hat{\boldsymbol{a}})= \begin{cases}\{1, \ldots, n\} & \text { if } \hat{\boldsymbol{a}} \in \Omega_{\mathcal{J}} \\ \emptyset & \text { else. }\end{cases}
$$

These selective aperture regions $\Omega_{\mathcal{J}}, \forall \mathcal{J} \in \mathfrak{I}$, define the choice of the index set $\mathcal{I}$ but not the integer solution required to define the mapping $\mathcal{S}(\cdot)$ for all non-empty index sets. Let $S_{z, \mathcal{J}}$ be a valid decision region of a partial integer estimator for the subset $\mathcal{J}$ as introduced in (4.1). The association with the index set $\mathcal{J}$ is written explicitly within this section, since the index set can assume different values. For the definition of a generalized integer aperture estimator, the decision regions $S_{\boldsymbol{z}, \mathcal{J}}$ for all possible non-empty index sets $\mathcal{J} \in \mathfrak{I}$ are required, since these are used to define the integer mapping once the index set has been determined. For each possible non-empty 
index set $\mathcal{J}$ we define

$$
\Omega_{\mathcal{J}, \boldsymbol{z}}=\Omega_{\mathcal{J}} \cap S_{\boldsymbol{z}, \mathcal{J}}, \quad \forall \boldsymbol{z} \in \mathbb{Z}^{|\mathcal{J}|} .
$$

With the decision regions $S_{\boldsymbol{z}, \mathcal{J}}, \forall \boldsymbol{z} \in \mathbb{Z}^{|\mathcal{J}|}$, covering the whole $\mathbb{R}^{n}$ without gaps and overlaps, it follows that $\Omega_{\mathcal{J}}=\bigcup_{z \in \mathbb{Z}|\mathcal{J}|} \Omega_{\mathcal{J}, \boldsymbol{z}}, \forall \mathcal{J} \in \mathfrak{I} \backslash \emptyset$. The integer estimate $\check{\boldsymbol{a}}$ of a generalized integer aperture estimator can now be explicitly written as

$$
\check{\boldsymbol{a}}= \begin{cases}\sum_{\boldsymbol{z} \in \mathbb{Z}|\mathcal{I}|} \omega_{\mathcal{I}, \boldsymbol{z}}(\hat{\boldsymbol{a}}) \boldsymbol{z} & \text { if } \mathcal{I} \neq \emptyset \\ {[]} & \text { if } \mathcal{I}=\emptyset\end{cases}
$$

with the random index set $\mathcal{I}$ as defined in (5.1). Evaluating (5.4) requires the indicator function $\omega_{\mathcal{J}, z}(\cdot)$ to be defined for all possible non-empty index sets, i.e., for all possible non-empty realizations of $\mathcal{I}$. It is given by

$$
\omega_{\mathcal{J}, \boldsymbol{z}}(\hat{\boldsymbol{a}})=\left\{\begin{array}{ll}
1 & \text { if } \hat{\boldsymbol{a}} \in \Omega_{\mathcal{J}, \boldsymbol{z}} \\
0 & \text { else }
\end{array}, \quad \forall \mathcal{J} \in \mathfrak{I} \backslash \emptyset .\right.
$$

The regions $\Omega_{\mathcal{J}, \boldsymbol{z}}$ define both the choice of the index set $\mathcal{I}$ and the corresponding integer solution $\check{\boldsymbol{a}} \in \mathbb{Z}^{|\mathcal{I}|}$. The following constraints have to be imposed when defining the selective aperture regions $\Omega_{\mathcal{J}}$ :

$$
\begin{aligned}
& \text { (i) } \bigcup_{\mathcal{J} \in \mathfrak{I}} \Omega_{\mathcal{J}}=\mathbb{R}^{n} \\
& \text { (ii) } \quad \operatorname{int}\left(\Omega_{\mathcal{J}}\right) \cap \operatorname{int}\left(\Omega_{\mathcal{K}}\right)=\emptyset, \quad \forall \mathcal{J}, \mathcal{K} \in \mathfrak{I}, \mathcal{J} \neq \mathcal{K} \\
& \text { (iii) } \Omega_{\mathcal{J}}=\Omega_{\mathcal{J}}+\boldsymbol{v}, \quad \forall \boldsymbol{v} \in \mathbb{Z}^{n}, \forall \mathcal{J} \in \mathfrak{I} .
\end{aligned}
$$

These constraints have some similarities to the ones of the pull-in regions of an integer estimator in (3.4), but with the regions being associated to different index sets instead of different integer vectors. The first two conditions ensure a unique index set for any float solution $\hat{\boldsymbol{a}} \in \mathbb{R}^{n}$ as defined in (5.1). The third condition states that if the float solution $\hat{\boldsymbol{a}}$ is shifted by an arbitrary integer vector $z \in \mathbb{Z}^{n}$, this must not affect the choice of the index set $\mathcal{I}$.

The class of partial integer estimators as introduced in Chapter 4, and therefore also the class of integer estimators, is contained in the class of integer aperture estimators and follows by choosing the selective aperture region of a specific non-empty index set $\mathcal{J}$ as $\Omega_{\mathcal{J}}=\mathbb{R}^{n}$, which leads to the deterministic index set $\mathcal{I}=\mathcal{J}$. Further, with $\mathfrak{I}=\{\emptyset,\{1, \ldots, n\}\}$, the class of generalized integer aperture estimators reduces to the class of integer aperture estimators. Generalized integer aperture estimators are thus the most flexible approach among the four discussed classes for estimating integer parameters.

A different yet equivalent formulation of a generalized integer aperture estimator is presented in Brack and Günther (2014). Let $S_{\boldsymbol{v}}, \forall \boldsymbol{v} \in \mathbb{Z}^{n}$, denote the pull-in regions of a valid integer estimator for estimating the full set of integers, and let $\tilde{\Omega}_{\mathcal{J}, \boldsymbol{v}}=\Omega_{\mathcal{J}} \cap S_{\boldsymbol{v}}, \forall \boldsymbol{v} \in \mathbb{Z}^{n}, \forall \mathcal{J} \in \mathfrak{I}$, with $\Omega_{\mathcal{J}}$ valid selective aperture regions. That is, the pull-in regions $S_{\boldsymbol{v}}$ of the $n$ dimensional integer vectors are further split up into sub-regions corresponding to each possible subset $\mathcal{J} \in \mathfrak{I}$

$$
S_{\boldsymbol{v}}=\bigcup_{\mathcal{J} \in \mathfrak{I}} \tilde{\Omega}_{\mathcal{J}, \boldsymbol{v}}, \quad \forall \boldsymbol{v} \in \mathbb{Z}^{n}
$$

Similarly, the selective aperture regions $\Omega_{\mathcal{J}}$ are composed of sub-regions corresponding to all integer vectors $\boldsymbol{v} \in \mathbb{Z}^{n}$

$$
\Omega_{\mathcal{J}}=\bigcup_{\boldsymbol{v} \in \mathbb{Z}^{n}} \tilde{\Omega}_{\mathcal{J}, \boldsymbol{v}}, \quad \forall \mathcal{J} \in \mathfrak{I}
$$


With the index set $\mathcal{I}$ as defined in (5.1), the integer estimate $\check{\boldsymbol{a}}$ as defined in (5.4) can alternatively be written as

$$
\check{\boldsymbol{a}}=\boldsymbol{\Gamma}_{\mathcal{I}} \sum_{\boldsymbol{v} \in \mathbb{Z}^{n}} \tilde{\omega}_{\mathcal{I}, \boldsymbol{v}}(\hat{\boldsymbol{a}}) \boldsymbol{v}
$$

where the multiplication of a vector $\boldsymbol{x} \in \mathbb{R}^{n}$ with $\boldsymbol{\Gamma}_{\mathcal{J}} \in\{0,1\}^{|\mathcal{J}| \times n}$ selects $\boldsymbol{x}_{\mathcal{J}}$ from $\boldsymbol{x}$. The indicator functions $\tilde{\omega}_{\mathcal{J}, v}(\cdot)$ for all possible index sets are given by

$$
\tilde{\omega}_{\mathcal{J}, \boldsymbol{v}}(\hat{\boldsymbol{a}})=\left\{\begin{array}{ll}
1 & \text { if } \hat{\boldsymbol{a}} \in \tilde{\Omega}_{\mathcal{J}, \boldsymbol{v}} \\
0 & \text { else }
\end{array}, \quad \forall \mathcal{J} \in \mathfrak{I} .\right.
$$

Essentially, with the properties of the pull-in regions in (3.4) and of the selective aperture regions in (5.6), the float solution $\hat{\boldsymbol{a}}$ falls within exactly one of the regions $\tilde{\Omega}_{\mathcal{J}, \boldsymbol{v}}$, which defines the integer subset $\mathcal{I}$ and-through selecting the entries $\boldsymbol{v}_{\mathcal{J}}$-also the integer estimate $\check{\boldsymbol{a}} \in \mathbb{Z}^{|\mathcal{I}|}$. This alternative formulation reveals a possible general strategy for formulating generalized integer aperture estimators: First, the full $n$ dimensional integer solution according to an integer estimator is computed. Then, the index set is determined, e.g., using one or more acceptance tests, and the corresponding entries of the previously determined integer solution are selected.

In order for the two representations to be identical, the regions that result in the same index set and integer solution have to be identical. With (5.4) and (5.9) this means that

$$
\Omega_{\mathcal{J}, \boldsymbol{z}}=\bigcup_{\boldsymbol{v} \in \mathbb{Z}^{n} \mid \boldsymbol{v}_{\mathcal{J}}=\boldsymbol{z}} \tilde{\Omega}_{\mathcal{J}, \boldsymbol{v}}, \quad \forall \mathcal{J} \in \mathfrak{I} .
$$

Using the definition of the regions $\Omega_{\mathcal{J}, \boldsymbol{z}}$ and $\tilde{\Omega}_{\mathcal{J}, \boldsymbol{v}}$, this translates into the following condition relating the regions $S_{\boldsymbol{z}, \mathcal{J}}, \forall \mathcal{J} \in \mathfrak{I}$, and $S_{\boldsymbol{v}}$

$$
\Omega_{\mathcal{J}} \cap S_{\boldsymbol{z}, \mathcal{J}}=\Omega_{\mathcal{J}} \cap\left(\bigcup_{\boldsymbol{v} \in \mathbb{Z}^{n} \mid \boldsymbol{v}_{\mathcal{J}}=\boldsymbol{z}} S_{\boldsymbol{v}}\right), \quad \forall \mathcal{J} \in \mathfrak{I} .
$$

Starting from either of the two representations, the corresponding alternative representation can thus be found by deriving decision regions $S_{\boldsymbol{z}, \mathcal{J}}, \forall \mathcal{J} \in \mathfrak{I}$, from the pull-in regions $S_{\boldsymbol{v}}$ or vice versa, such that (5.12) is met. The specific realization of the generalized integer aperture estimator determines which of the two different representations is more intuitive to work with.

\subsection{Quality of the Estimates}

Since the same integer vector $\check{\boldsymbol{a}}$ may be obtained for different realizations of the index set $\mathcal{I}$, the pmf of $\check{\boldsymbol{a}}$ is only meaningful when it is considered jointly with the pmf of $\mathcal{I}$. With $p_{\hat{\boldsymbol{a}}}(\boldsymbol{x})$ the pdf of the float estimate $\hat{\boldsymbol{a}}$ and the selective aperture decision regions $\Omega_{\mathcal{J}, z}$, the joint pmf of $\check{\boldsymbol{a}}$ and $\mathcal{I}$ follows as

$$
\begin{aligned}
P(\mathcal{I}=\mathcal{J}, \check{\boldsymbol{a}}=\boldsymbol{z}) & =P\left(\hat{\boldsymbol{a}} \in \Omega_{\mathcal{J}, \boldsymbol{z}}\right)=\int_{\Omega_{\mathcal{J}, \boldsymbol{z}}} p_{\hat{\boldsymbol{a}}}(\boldsymbol{x}) \mathrm{d} \boldsymbol{x}, \quad \forall \mathcal{J} \in \mathfrak{I} \backslash \emptyset, \boldsymbol{z} \in \mathbb{Z}^{|\mathcal{J}|} \\
\text { and, if } \emptyset \in \mathfrak{I}, \quad P(\check{\boldsymbol{a}}=[]) & =P\left(\hat{\boldsymbol{a}} \in \Omega_{\emptyset}\right)=\int_{\Omega_{\emptyset}} p_{\hat{\boldsymbol{a}}}(\boldsymbol{x}) \mathrm{d} \boldsymbol{x} .
\end{aligned}
$$


For the alternative representation with the regions $\tilde{\Omega}_{\mathcal{J}, v}$ we simply use the relation (5.11) when evaluating (5.13). The probabilities of resolving specific subsets $\mathcal{J}$ of the integer parameters is given by the pmf of $\mathcal{I}$

$$
P(\mathcal{I}=\mathcal{J})=\sum_{\boldsymbol{z} \in \mathbb{Z}|\mathcal{J}|} P(\mathcal{I}=\mathcal{J}, \check{\boldsymbol{a}}=\boldsymbol{z})=\int_{\Omega_{\mathcal{J}}} p_{\hat{\boldsymbol{a}}}(\boldsymbol{x}) \mathrm{d} \boldsymbol{x}, \quad \forall \mathcal{J} \in \mathfrak{I} \backslash \emptyset
$$

and, if $\emptyset \in \mathfrak{I}, \quad P(\mathcal{I}=\emptyset)=P(\check{\boldsymbol{a}}=[])$.

Like for the class of integer aperture estimators, three cases can be distinguished when analyzing the quality of the estimates $\check{\boldsymbol{a}}$ of a generalized integer aperture estimator:

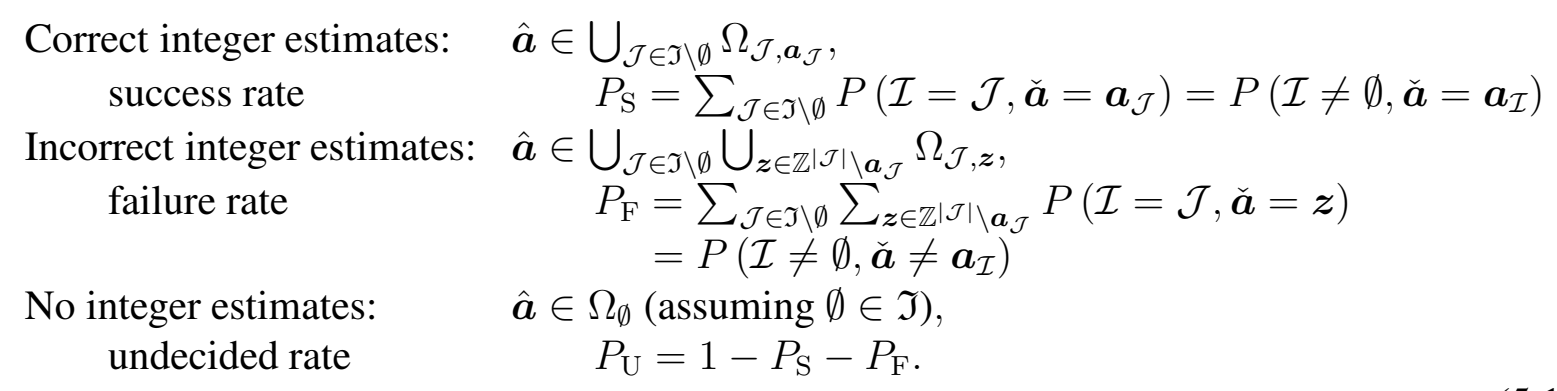

That is, whenever all integer parameters with an index $i \in \mathcal{I}$ are resolved correctly, given that at least one integer parameter is resolved at all, this event is called success, whereas if only a single integer parameter is resolved incorrectly, the event is called a failure. The third option no integer estimates refers to the case that $\mathcal{I}=\emptyset$, meaning that no integer parameter is resolved.

The distribution of the fixed estimates $\breve{b}$ of the real valued parameters as defined in (2.84) can be derived similarly as in Section 4.2 and is given by

$$
\begin{array}{r}
p_{\breve{\boldsymbol{b}}}(\boldsymbol{\beta})=\sum_{\mathcal{J} \in \mathcal{T} \backslash \emptyset} \sum_{\boldsymbol{z} \in \mathbb{Z}|\mathcal{J}|} \int_{\Omega_{\mathcal{J}, \boldsymbol{z}}} p_{\hat{\boldsymbol{b}}, \hat{\boldsymbol{a}}_{\overline{\mathcal{J}}} \mid \hat{\boldsymbol{a}}_{\mathcal{J}}}\left(\boldsymbol{\beta}+\boldsymbol{Q}_{\hat{\boldsymbol{b}} \hat{\boldsymbol{a}}_{\mathcal{J}}} \boldsymbol{Q}_{\hat{\boldsymbol{a}}_{\mathcal{J}}}^{-1}\left(\boldsymbol{x}_{\mathcal{J}}-\boldsymbol{z}\right), \boldsymbol{x}_{\overline{\mathcal{J}}} \mid \boldsymbol{x}_{\mathcal{J}}\right) p_{\hat{\boldsymbol{a}}_{\mathcal{J}}}\left(\boldsymbol{x}_{\mathcal{J}}\right) \mathrm{d} \boldsymbol{x} \\
+\int_{\Omega_{\emptyset}} p_{\hat{\boldsymbol{b}} \mid \hat{\boldsymbol{a}}}(\boldsymbol{\beta} \mid \boldsymbol{x}) p_{\hat{\boldsymbol{a}}}(\boldsymbol{x}) \mathrm{d} \boldsymbol{x}
\end{array}
$$

where the first part describes the case that a non-empty set $\mathcal{J}$ of the integer parameters is resolved, for each of which we have to sum over all possible $|\mathcal{J}|$ dimensional integer vectors. The second part refers to $\mathcal{I}=\emptyset$ and only exists for $\emptyset \in \mathfrak{I}$. The interpretation of (5.16) is not very intuitive. According to Section 4.2, if the index set $\mathcal{I}$ would be deterministic and the success rate sufficiently large, i.e., if a single one of the integrals in the sum in (5.16) would have the domain $\mathbb{R}^{n}$ and all others an empty domain, $\check{b}$ would follow a Gaussian distribution with mean value $\boldsymbol{b}$ and covariance matrix $\boldsymbol{Q}_{\hat{b} \mid \hat{\boldsymbol{a}}_{\mathcal{J}}}$ corresponding to the given set. Since this is not the case, the distribution of $\breve{b}$ is, however, rather complicated. If the failure rate $P_{\mathrm{F}}$ is sufficiently small, we can still use $\boldsymbol{Q}_{\hat{\boldsymbol{b}} \mid \hat{\boldsymbol{a}}_{\mathcal{J}}}$ as a rough indicator for the precision that we can expect for $\breve{b}$ once the value of the index set $\mathcal{I}$ has been determined. Let the function $g(\cdot): \mathbb{R}^{n} \mapsto \mathbb{R}^{p}$ be defined such that $g(\hat{\boldsymbol{a}})=\boldsymbol{Q}_{\hat{b} \hat{\boldsymbol{a}}_{\mathcal{I}}} \boldsymbol{Q}_{\hat{\boldsymbol{a}}_{\mathcal{I}}}^{-1}\left(\hat{\boldsymbol{a}}_{\mathcal{I}}-\check{\boldsymbol{a}}\right)$. According to (2.85) the estimates $\breve{b}$ are unbiased if $\mathrm{E}[g(\hat{\boldsymbol{a}})]=\mathbf{0}$. This expectation can be worked 
out as

$$
\begin{aligned}
\mathrm{E}[g(\hat{\boldsymbol{a}})] & =\int_{\mathbb{R}^{n}} g(\boldsymbol{x}) p_{\hat{\boldsymbol{a}}}(\boldsymbol{x}) \mathrm{d} \boldsymbol{x} \\
& =\sum_{\mathcal{J} \in \mathcal{I} \backslash \emptyset} \boldsymbol{Q}_{\hat{\boldsymbol{b}} \hat{\boldsymbol{a}}_{\mathcal{J}}} \boldsymbol{Q}_{\hat{\boldsymbol{a}}_{\mathcal{J}}}^{-1} \sum_{\boldsymbol{z} \in \mathbb{Z}|\mathcal{J}|} \int_{\Omega_{\mathcal{J}, \boldsymbol{z}}}\left(\boldsymbol{x}_{\mathcal{J}}-\boldsymbol{z}\right) p_{\hat{\boldsymbol{a}}}(\boldsymbol{x}) \mathrm{d} \boldsymbol{x},
\end{aligned}
$$

so that $\check{b}$ is for instance unbiased, if the second sum cancels for all non-empty realizations $\mathcal{J}$ of the

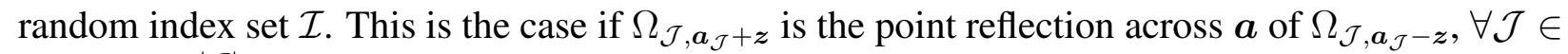
$\mathfrak{I} \backslash \emptyset, \boldsymbol{z} \in \mathbb{Z}^{|\mathcal{J}|}$, or, equivalently, if $\Omega_{\mathcal{J}, \boldsymbol{z}}$ is symmetric with respect to $\boldsymbol{v} \in \mathbb{Z}^{n}$ with $\boldsymbol{v}_{\mathcal{J}}=\boldsymbol{z}$. This can easily be demonstrated, since the symmetry implies that $2 \boldsymbol{a}-\boldsymbol{x} \in \Omega_{\mathcal{J}, \boldsymbol{a}_{\mathcal{J}}-\boldsymbol{z}}$ if $\boldsymbol{x} \in \Omega_{\mathcal{J}, \boldsymbol{a}_{\mathcal{J}}+\boldsymbol{z}}$. With $p_{\hat{\boldsymbol{a}}}(\boldsymbol{x})$ being symmetric with respect to $\boldsymbol{a}$, the sum of the two integrals over $\Omega_{\mathcal{J}, \boldsymbol{a}_{\mathcal{J}}-\boldsymbol{z}}$ and $\Omega_{\mathcal{J}, \boldsymbol{a}_{\mathcal{J}}+\boldsymbol{z}}$ then cancels for any $\boldsymbol{z} \in \mathbb{Z}^{|\mathcal{J}|} \backslash \mathbf{0}$, and the integral over $\Omega_{\mathcal{J}, \boldsymbol{a}_{\mathcal{J}}}$ is zero, so that $\mathrm{E}[g(\hat{\boldsymbol{a}})]=\mathbf{0}$.

As discussed earlier, our goal is to limit the ambiguity failure rate $P_{\mathrm{F}}$ in GNSS positioning to a maximum tolerable value. With the concept of generalized integer aperture estimation the failure rate can be fully controlled by jointly formulating the selective aperture decision regions $\Omega_{\mathcal{J}, \boldsymbol{z}}$, so that a maximum failure rate can be guaranteed irrespective of the strength of the underlying system model. Thereby, the probability of resolving at least one ambiguity parameter is given by $P(\mathcal{I} \neq \emptyset)=P_{\mathrm{S}}+P_{\mathrm{F}}$. This fixing rate, however, is by itself not a suitable measure to describe and compare the performance of different generalized integer aperture estimators, since it does not reflect which ambiguity subsets are resolved. When used with a user-defined maximum tolerable failure rate, generalized integer aperture estimators are the suitable tool for data-driven reliable partial ambiguity resolution, where both the description of the system model and the measurement data is used to choose the ambiguity subset to be resolved.

\subsection{Single-Subset Generalized Integer Aperture Estimators}

One strategy for designing a generalized integer aperture estimator is to choose the set of possible index sets $\mathcal{I}$ as $\mathfrak{I}=\left\{\emptyset, \mathcal{I}^{\prime}\right\}$, with $\mathcal{I}^{\prime}$ any of the $2^{n}-1$ non-empty options. That is, either an a priori defined subset $\mathcal{I}^{\prime}$ of the integer parameters $\boldsymbol{a}$ is resolved, or none of them is. The probability of resolving the subset $\mathcal{I}^{\prime}$ of the integer parameters is then simply $P\left(\mathcal{I}=\mathcal{I}^{\prime}\right)=P_{\mathrm{S}}+P_{\mathrm{F}}$ and $P(\mathcal{I}=\emptyset)=1-P\left(\mathcal{I}=\mathcal{I}^{\prime}\right)=P_{\mathrm{U}}$. This strategy is very similar to conventional integer aperture estimation, for which the index set can assume the two values $\mathcal{I}=\emptyset$ or $\mathcal{I}=\{1, \ldots, n\}$, cf. Section 3.2. In the following, the optimal generalized integer aperture estimator for $\mathfrak{I}=\left\{\emptyset, \mathcal{I}^{\prime}\right\}$ and an arbitrary choice of $\mathcal{I}^{\prime}$ as well as a difference test based realization are presented, and the selection of the index set $\mathcal{I}^{\prime}$ for GNSS applications is discussed.

\subsubsection{Optimal Single-Subset Generalized Integer Aperture Estimation}

Analogously to the optimal integer aperture estimator in Section 3.2.2, the optimal single-subset generalized integer aperture estimator with $\mathfrak{I}=\left\{\emptyset, \mathcal{I}^{\prime}\right\}$ is defined as the solution of the optimization problem

$$
\max _{\Omega_{\mathcal{I}^{\prime}, \boldsymbol{z}}} P_{\mathrm{S}} \text { s.t. } \quad P_{\mathrm{F}}=\gamma,
$$

with $\Omega_{\mathcal{I}^{\prime}, \boldsymbol{z}}$ a valid selective aperture decision region and the success and failure rates $P_{\mathrm{S}}$ and $P_{\mathrm{F}}$ as defined in (5.15). It is noted that the complementary point set is simply $\mathbb{R}^{n} \backslash \Omega_{\mathcal{I}^{\prime}}=\Omega_{\emptyset}$. That is, among all generalized aperture estimators with $\mathfrak{I}=\left\{\emptyset, \mathcal{I}^{\prime}\right\}$, the optimal estimator leads to the 
highest probability of resolving the subset $\mathcal{I}^{\prime}$ of the integer parameters for any given failure rate $P_{\mathrm{F}}=\gamma$. The selective aperture decision regions $\Omega_{\mathcal{I}^{\prime}, \boldsymbol{z}}$ of the optimal generalized integer aperture estimator are given by

$$
\begin{array}{r}
\Omega_{\mathcal{I}^{\prime}, \boldsymbol{z}}=\left\{\boldsymbol{x} \in S_{\boldsymbol{z}, \mathcal{I}^{\prime}} \mid \sum_{\boldsymbol{v} \in \mathbb{Z}^{n} \mid \boldsymbol{v}_{\mathcal{I}^{\prime}} \neq \boldsymbol{z}} \exp \left(-\frac{1}{2}\|\boldsymbol{x}-\boldsymbol{v}\|_{\boldsymbol{Q}_{\hat{a}}}^{2}\right) \leq \mu \sum_{\boldsymbol{v} \in \mathbb{Z}^{n} \mid \boldsymbol{v}_{\mathcal{I}^{\prime}}=\boldsymbol{z}} \exp \left(-\frac{1}{2}\|\boldsymbol{x}-\boldsymbol{v}\|_{\boldsymbol{Q}_{\hat{\boldsymbol{a}}}}^{2}\right)\right\}, \\
\forall \boldsymbol{z} \in \mathbb{Z}^{\left|\mathcal{I}^{\prime}\right|},
\end{array}
$$

where $S_{\boldsymbol{z}, \mathcal{I}^{\prime}}$ are the decision regions of the optimal partial integer estimator for $\mathcal{I}=\mathcal{I}^{\prime}$ as defined in (4.17) and the critical value $\mu$ is chosen so as to obtain a fixed failure rate of $P_{\mathrm{F}}=\gamma$. The proof is as follows. With $p_{\hat{\boldsymbol{a}}}(\boldsymbol{x})$ the Gaussian pdf of the float solution and definition (5.15), the optimization problem (5.18) can equivalently be written as

$$
\max _{\Omega_{\mathcal{I}^{\prime}, \boldsymbol{z}}} \int_{\Omega_{\mathcal{I}^{\prime}}} p_{\hat{\boldsymbol{a}}}(\boldsymbol{x}) \mathrm{d} \boldsymbol{x} \quad \text { s.t. } \quad \sum_{\boldsymbol{u} \in \mathbb{Z}^{\mid \mathcal{I}^{\prime} \backslash} \backslash \boldsymbol{a}_{\mathcal{I}^{\prime}}} \int_{\Omega_{\mathcal{I}^{\prime}, \boldsymbol{u}}} p_{\hat{\boldsymbol{a}}}(\boldsymbol{x}) \mathrm{d} \boldsymbol{x}=\gamma .
$$

Since $\Omega_{\mathcal{I}^{\prime}, \boldsymbol{z}}=\Omega_{\mathcal{I}^{\prime}} \cap S_{\boldsymbol{z}, \mathcal{I}^{\prime}}$, the solution of this problem can be split into two steps. First, the optimal selective aperture region $\Omega_{\mathcal{I}^{\prime}}^{*}$ is derived for a given partial integer estimator as defined by the decision regions $S_{\boldsymbol{z}, \mathcal{I}^{\prime}}$. In the second step, the optimal choice of $S_{\boldsymbol{z}, \mathcal{I}^{\prime}}$ is discussed. Let $s_{\boldsymbol{u}, \mathcal{I}^{\prime}}(\cdot)$ be the indicator function of the given partial integer estimator for the subset $\mathcal{I}^{\prime}$, then the first problem is given by

$$
\max _{\Omega_{\mathcal{I}^{\prime}}} \int_{\Omega_{\mathcal{I}^{\prime}}} p_{\hat{\boldsymbol{a}}}(\boldsymbol{x}) \mathrm{d} \boldsymbol{x} \quad \text { s.t. } \quad \int_{\Omega_{\mathcal{I}^{\prime}}} \sum_{\boldsymbol{u} \in \mathbb{Z}^{\mid \mathcal{I}^{\prime} \backslash} \backslash \boldsymbol{a}_{\mathcal{I}^{\prime}}} p_{\hat{\boldsymbol{a}}}(\boldsymbol{x}) s_{\boldsymbol{u}, \mathcal{I}^{\prime}}(\boldsymbol{x}) \mathrm{d} \boldsymbol{x}=\gamma .
$$

This optimization problem can be solved by means of applying a modified version of the NeymanPearson Lemma that accounts for the property of integer translational invariance of the region $\Omega_{\mathcal{I}^{\prime}}$, see property ( iii) in (5.6). It is derived in Teunissen (2005a) and reads:

With $f(\boldsymbol{x})$ and $g(\boldsymbol{x})$ two functions that are integrable over $\mathbb{R}^{n}$, the region

$$
\Omega^{*}=\left\{\boldsymbol{x} \in \mathbb{R}^{n} \mid \sum_{\boldsymbol{v} \in \mathbb{Z}^{n}} f(\boldsymbol{x}+\boldsymbol{v}) \geq \lambda \sum_{\boldsymbol{v} \in \mathbb{Z}^{n}} g(\boldsymbol{x}+\boldsymbol{v})\right\},
$$

with $\lambda \in \mathbb{R}$, solves the constrained maximization problem

$$
\begin{array}{lll}
\max _{\Omega} \int_{\Omega} f(\boldsymbol{x}) \mathrm{d} \boldsymbol{x} \quad \text { s.t. } & \int_{\Omega} g(\boldsymbol{x}) \mathrm{d} \boldsymbol{x}=\text { const. } \\
& & \Omega=\Omega+\boldsymbol{v}, \forall \boldsymbol{v} \in \mathbb{Z}^{n}
\end{array}
$$

if $\lambda$ is chosen so as to satisfy the first constraint.

The optimal selective aperture region $\Omega_{\mathcal{I}^{\prime}}^{*}$ follows from applying this Lemma to (5.21) as

$$
\Omega_{\mathcal{I}^{\prime}}^{*}=\left\{\boldsymbol{x} \in \mathbb{R}^{n} \mid \sum_{\boldsymbol{v} \in \mathbb{Z}^{n}} p_{\hat{\boldsymbol{a}}}(\boldsymbol{x}+\boldsymbol{v}) \geq \lambda \sum_{\boldsymbol{v} \in \mathbb{Z}^{n}} \sum_{\boldsymbol{u} \in \mathbb{Z}^{\mid \mathcal{I}^{\prime} \backslash \backslash \boldsymbol{a}_{\mathcal{I}^{\prime}}}} p_{\hat{\boldsymbol{a}}}(\boldsymbol{x}+\boldsymbol{v}) s_{\boldsymbol{u}, \mathcal{I}^{\prime}}(\boldsymbol{x}+\boldsymbol{v})\right\} .
$$

From property (iii) of the decision regions $S_{\boldsymbol{u}, \mathcal{I}^{\prime}}$ of a partial integer estimator in (4.4) we know that $s_{\boldsymbol{u}, \mathcal{I}^{\prime}}(\boldsymbol{x}+\boldsymbol{v})=s_{\boldsymbol{u}-\boldsymbol{v}_{\mathcal{I}^{\prime}}, \mathcal{I}^{\prime}}(\boldsymbol{x}), \forall \boldsymbol{v} \in \mathbb{Z}^{n}$. For a given value $\boldsymbol{v} \in \mathbb{Z}^{n}$, the last sum in (5.24) can therefore be rewritten as

$$
\sum_{\boldsymbol{u} \in \mathbb{Z}^{\mid \mathcal{I}^{\prime} \backslash \backslash \boldsymbol{a}_{\mathcal{I}^{\prime}}}} p_{\hat{\boldsymbol{a}}}(\boldsymbol{x}+\boldsymbol{v}) s_{\boldsymbol{u}, \mathcal{I}^{\prime}}(\boldsymbol{x}+\boldsymbol{v})=\left\{\begin{array}{ll}
p_{\hat{\boldsymbol{a}}}(\boldsymbol{x}+\boldsymbol{v}) & \text { if } \boldsymbol{v}_{\mathcal{I}^{\prime}} \neq \boldsymbol{a}_{\mathcal{I}^{\prime}}-\boldsymbol{z} \\
0 & \text { else }
\end{array}, \quad \forall \boldsymbol{x} \in S_{\boldsymbol{z}, \mathcal{I}^{\prime}}\right.
$$


Then,

$$
\begin{aligned}
\sum_{\boldsymbol{v} \in \mathbb{Z}^{n}} \sum_{\boldsymbol{u} \in \mathbb{Z}^{\left|\mathcal{I}^{\prime}\right| \backslash \boldsymbol{a}_{\mathcal{I}^{\prime}}}} p_{\hat{\boldsymbol{a}}}(\boldsymbol{x}+\boldsymbol{v}) s_{\boldsymbol{u}, \mathcal{I}^{\prime}}(\boldsymbol{x}+\boldsymbol{v}) & =\sum_{\boldsymbol{v} \in \mathbb{Z}^{n} \mid \boldsymbol{v}_{\mathcal{I}^{\prime}} \neq \boldsymbol{a}_{\mathcal{I}^{\prime}}-\boldsymbol{z}} p_{\hat{\boldsymbol{a}}}(\boldsymbol{x}+\boldsymbol{v}) \\
& =\sum_{\boldsymbol{v} \in \mathbb{Z}^{n} \mid \boldsymbol{v}_{\mathcal{I}^{\prime}} \neq \boldsymbol{z}} p_{\hat{\boldsymbol{a}}}(\boldsymbol{x}+\boldsymbol{a}-\boldsymbol{v}) .
\end{aligned}
$$

With $\Omega_{\mathcal{I}^{\prime}, \boldsymbol{z}}^{*}=\Omega_{\mathcal{I}^{\prime}}^{*} \cap S_{\boldsymbol{z}, \mathcal{I}^{\prime}}$ this finally results in

$$
\begin{aligned}
\Omega_{\mathcal{I}^{\prime}, \boldsymbol{z}}^{*} & =\left\{\boldsymbol{x} \in S_{\boldsymbol{z}, \mathcal{I}^{\prime}} \mid \sum_{\boldsymbol{v} \in \mathbb{Z}^{n}} p_{\hat{\boldsymbol{a}}}(\boldsymbol{x}+\boldsymbol{a}-\boldsymbol{v}) \geq \lambda \sum_{\boldsymbol{v} \in \mathbb{Z}^{n} \mid \boldsymbol{v}_{\mathcal{I}^{\prime}} \neq \boldsymbol{z}} p_{\hat{\boldsymbol{a}}}(\boldsymbol{x}+\boldsymbol{a}-\boldsymbol{v})\right\} \\
& =\left\{\boldsymbol{x} \in S_{\boldsymbol{z}, \mathcal{I}^{\prime}} \mid \sum_{\boldsymbol{v} \in \mathbb{Z}^{n} \mid \boldsymbol{v}_{\mathcal{I}^{\prime}} \neq \boldsymbol{z}} p_{\hat{\boldsymbol{a}}}(\boldsymbol{x}+\boldsymbol{a}-\boldsymbol{v}) \leq \mu \sum_{\boldsymbol{v} \in \mathbb{Z}^{n} \mid \boldsymbol{v}_{\mathcal{I}^{\prime}}=\boldsymbol{z}} p_{\hat{\boldsymbol{a}}}(\boldsymbol{x}+\boldsymbol{a}-\boldsymbol{v})\right\},
\end{aligned}
$$

where the critical value $\mu=\frac{1}{\lambda-1}$ has to be chosen so as to satisfy the fixed failure rate constraint. This tells us how to choose $\Omega_{\mathcal{I}^{\prime}}$ for an arbitrary $S_{\boldsymbol{z}, \mathcal{I}^{\prime}}$. For any selective aperture region $\Omega_{\mathcal{I}^{\prime}}$ the probability $P\left(\mathcal{I}=\mathcal{I}^{\prime}\right)=P\left(\hat{\boldsymbol{a}} \in \Omega_{\mathcal{I}^{\prime}}\right)$ is independent of the choice of $S_{\boldsymbol{z}, \mathcal{I}^{\prime}}$. Since $P\left(\hat{\boldsymbol{a}} \in \Omega_{\mathcal{I}^{\prime}}\right)$ is also the sum of the probabilities of correct and incorrect integer estimates for $\mathfrak{I}=\left\{\emptyset, \mathcal{I}^{\prime}\right\}$, any choice of $S_{\boldsymbol{z}, \mathcal{I}^{\prime}}$ that increases the success rate leads to a smaller failure rate. The optimal choice for the decision regions $S_{\boldsymbol{z}, \mathcal{I}^{\prime}}$ in (5.27) are consequently the ones of the optimal partial integer estimator from Section 4.3.3. Inserting the explicit expression of the Gaussian distribution in (5.27) concludes the proof.

For $\mathcal{I}^{\prime}=\{1, \ldots, n\}$, the sum on the right side of the condition in the definition of the optimal single-subset generalized integer aperture estimator (5.19) contains only one term and the estimator becomes identical to the optimal integer aperture estimator, cf. (3.30). The evaluation of whether or not the float solution $\hat{\boldsymbol{a}}$ lies within $\Omega_{\mathcal{I}^{\prime}}$ generally includes the evaluation of two infinite sums. These cannot be computed exactly, but have to be approximated, e.g., by omitting all terms whose contribution is sufficiently small. A possible choice for how to select the finite sets of $n$ dimensional integer vectors that are used instead of $\mathbb{Z}^{n}$ in (5.19) is discussed in Section 4.3.4 for computing the optimal partial integer estimator. For a given index set $\mathcal{I}^{\prime}$, the optimal single-subset generalized integer aperture estimator can now be computed by first determining the optimal partial integer estimator $\check{\boldsymbol{a}}_{\text {opt }}$ for $\mathcal{I}^{\prime}$. The same set of $n$ dimensional integer vectors that is already available from computing $\check{\boldsymbol{a}}_{\text {opt }}$ can then be used to evaluate the condition in (5.19) for the region $\Omega_{\mathcal{I}^{\prime}, \check{a}_{\text {opt }}}$. Methods for determining model dependent critical values $\mu$ for a fixed failure rate implementation are discussed in Section 5.5. The regions $\Omega_{\mathcal{I}^{\prime}, \boldsymbol{z}}$ of the optimal single-subset generalized integer aperture estimator are shown in Figure 5.1 for a two dimensional example.

A second example for which the variance of the first component of the float solution $\hat{\boldsymbol{a}}$ is drastically higher than the absolute value of the other elements of $\boldsymbol{Q}_{\hat{a}}$ is shown in Figure 5.2 for the optimal single-subset generalized integer aperture estimator, where the regions $\Omega_{\mathcal{I}^{\prime}}$ are constructed such that the probability of an incorrect integer estimate is identical in each subfigure. It is almost always possible to accept an integer solution for the second integer parameter, i.e., for $\mathcal{I}^{\prime}=\{2\}$, whereas resolving the first or even both integer parameters is very unlikely.

\subsubsection{Difference Test Based Single-Subset Generalized Integer Aperture Estimation}

A possible suboptimal strategy is to replace the infinite sums defining the optimal selective aperture decision regions in (5.19) by their respective maximum terms. In communication theory, the 

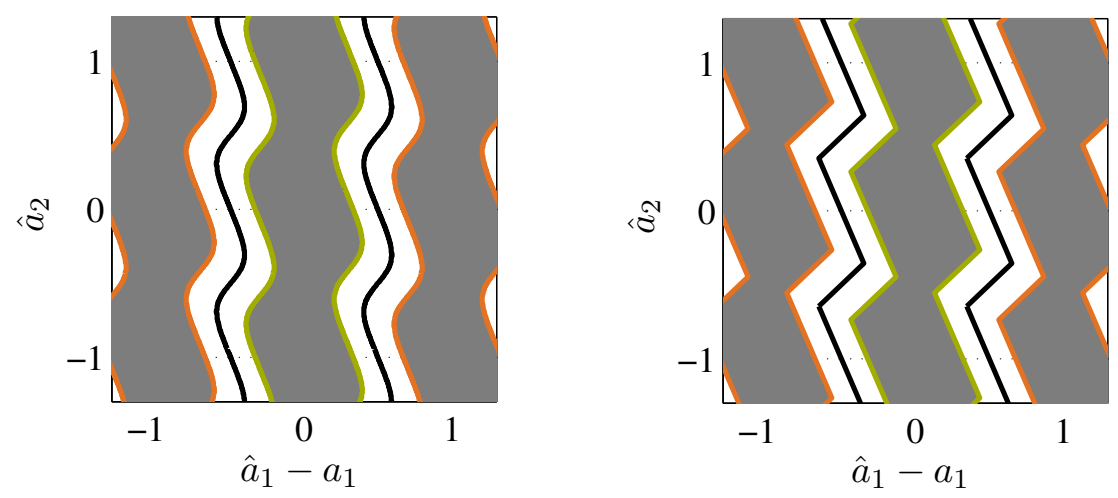

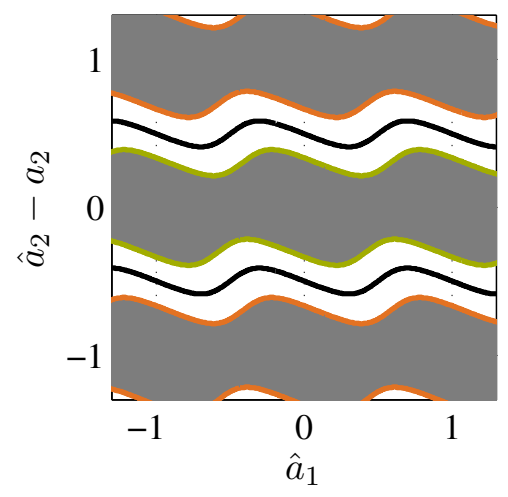

(a) Optimal single-subset

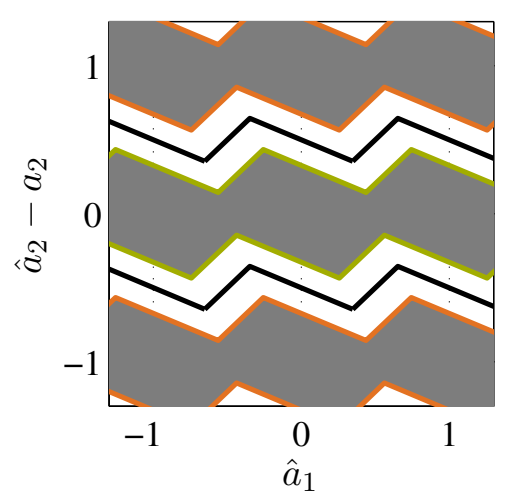

(b) Difference test single-subset

Figure 5.1: The selective aperture decision regions $\Omega_{\mathcal{I}^{\prime}, \boldsymbol{z}}$ are shown in gray for a two dimensional example with $\mathfrak{I}=\left\{\emptyset, \mathcal{I}^{\prime}\right\}$, where $\mathcal{I}^{\prime}=\{1\}$ in the first row and $\mathcal{I}^{\prime}=\{2\}$ in the second row. Green and orange edges indicate correct and incorrect integer estimates, respectively. The boundaries of the decision regions $S_{\boldsymbol{z}, \mathcal{I}^{\prime}}$ of the underlying partial integer estimators are drawn in black.

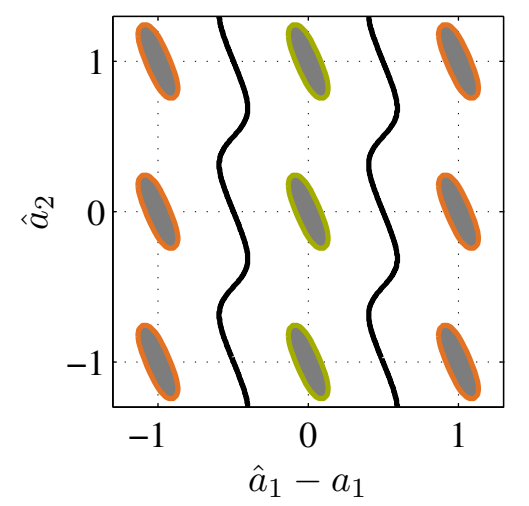

(a) $\mathfrak{I}=\{\emptyset,\{1\}\}$

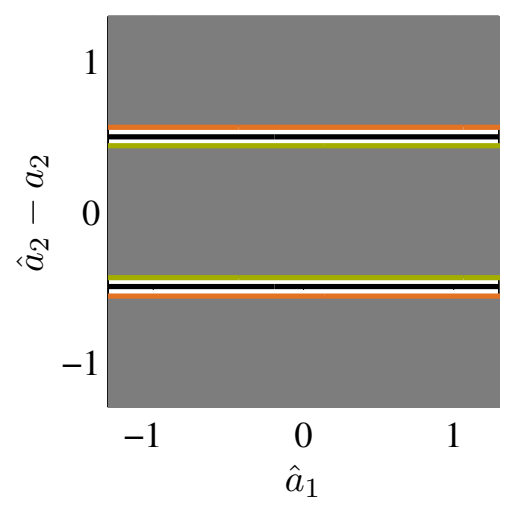

(b) $\mathfrak{I}=\{\emptyset,\{2\}\}$

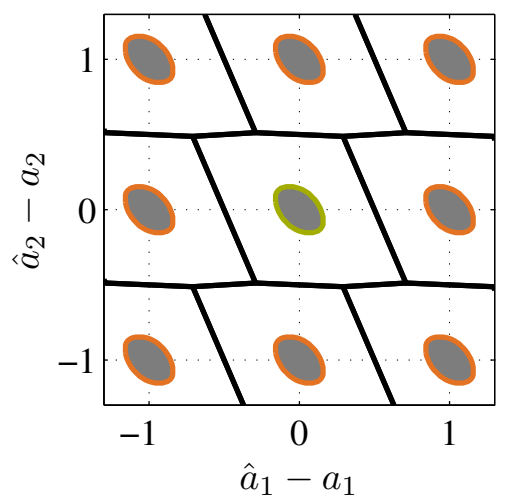

(c) $\mathfrak{I}=\{\emptyset,\{1,2\}\}$

Figure 5.2: The selective aperture regions $\Omega_{\mathcal{I}^{\prime}, \boldsymbol{z}}$ of the optimal single-subset generalized integer aperture estimator are shown in gray for a two dimensional example, where green and orange edges indicate correct and incorrect integer estimates, respectively. The boundaries of the decision regions $S_{\boldsymbol{z}, \mathcal{I}^{\prime}}$ of the underlying optimal partial integer estimator are drawn in black. 
condition in (5.19) is usually computed in the logarithmic domain, and this technique is therefore referred to as max-log approximation (Robertson et al. 1995; Hochwald and Ten Brink 2003; Steingrimsson et al. 2003). Using the same approximation for the underlying partial integer estimator means that the optimal partial integer estimator is replaced by full integer least-squares followed by a selection of entries as discussed in Section 4.3.2. The remaining term on the right side of the condition in (5.19) corresponds then to the full integer least-squares solution. The integer corresponding to remaining term on the left side of (5.19) can be referred to as the best counter hypothesis for the index set $\mathcal{I}^{\prime}$. It is defined as the integer vector closest to $\hat{a}$ that is different from the overall closest integer vector $\check{\boldsymbol{a}}_{\mathrm{ILS}}$ for at least one component of the index set $\mathcal{I}^{\prime}$ and given by

$$
\overline{\boldsymbol{a}}^{\mathcal{I}^{\prime}}=\underset{\boldsymbol{v} \in \mathbb{Z}^{n} \mid \boldsymbol{v}_{\mathcal{I}^{\prime}} \neq \check{\boldsymbol{a}}_{\mathrm{ILS}, \mathcal{I}^{\prime}}}{\operatorname{argmin}}\|\hat{\boldsymbol{a}}-\boldsymbol{v}\|_{\boldsymbol{Q}_{\hat{\boldsymbol{a}}}}^{2}
$$

The entries $\boldsymbol{\Gamma}_{\mathcal{I}^{\prime}} \check{\boldsymbol{a}}_{\mathrm{ILS}}$ of the integer least-squares solution corresponding to $\mathcal{I}^{\prime}$ are accepted as an integer estimate if

$$
\left\|\hat{\boldsymbol{a}}-\overline{\boldsymbol{a}}^{\mathcal{I}^{\prime}}\right\|_{\boldsymbol{Q}_{\hat{a}}}^{2}-\left\|\hat{\boldsymbol{a}}-\check{\boldsymbol{a}}_{\mathrm{ILS}}\right\|_{\boldsymbol{Q}_{\hat{a}}}^{2} \geq \mu
$$

with the critical value $\mu$. This test statistic is almost identical to the conventional difference test (3.32), but with the overall best counter-hypothesis $\overline{\boldsymbol{a}}$ being replaced by $\overline{\boldsymbol{a}}^{\mathcal{I}^{\prime}}$. The values produced by this modified difference test are always non-negative. This combined estimation and validation strategy is a generalized integer aperture estimator defined by the selective aperture decision regions

$$
\Omega_{\mathcal{I}^{\prime}, \boldsymbol{z}}=\left\{\boldsymbol{x} \in S_{\boldsymbol{z}, \mathcal{I}^{\prime}}\left|\|\boldsymbol{x}-\boldsymbol{u}\|_{\boldsymbol{Q}_{\hat{a}}}^{2}-\min _{\boldsymbol{v} \in \mathbb{Z}^{n}}\|\boldsymbol{x}-\boldsymbol{v}\|_{\boldsymbol{Q}_{\hat{a}}}^{2} \geq \mu, \forall \boldsymbol{u} \in \mathbb{Z}^{n}\right| \boldsymbol{u}_{\mathcal{I}^{\prime}} \neq \boldsymbol{z}\right\}, \quad \forall \boldsymbol{z} \in \mathbb{Z}^{\left|\mathcal{I}^{\prime}\right|}
$$

with $S_{\boldsymbol{z}, \mathcal{I}^{\prime}}$ the decision regions of full integer least-squares + selection. With $\Omega_{\emptyset}=\mathbb{R}^{n} \backslash \Omega_{\mathcal{I}^{\prime}}$, the first two conditions in (5.6) are already fulfilled. Due to the regularity of the integer grid, a shift of the float solution by an arbitrary integer $\boldsymbol{u} \in \mathbb{Z}^{n}$ shifts both the integer least-squares solution $\check{\boldsymbol{a}}_{\mathrm{ILS}}$ and the counter hypothesis $\overline{\boldsymbol{a}}^{\mathcal{I}^{\prime}}$ by the same integer, i.e., the test value (5.29) remains unchanged and the third condition is satisfied.

The performance of this difference test based approximation can be expected to be close to optimal if the distribution of the float solution $\hat{\boldsymbol{a}}$ is sufficiently peaked. It is noted that the integer least-squares solution $\check{\boldsymbol{a}}_{\mathrm{ILS}}$ can be equivalently computed for any integer transformation $Z$, but not the counter hypothesis $\overline{\boldsymbol{a}}^{\mathcal{I}^{\prime}}$, since the best counter hypothesis for one specific choice of $\boldsymbol{Z}$ is generally not the best or even only a valid counter hypothesis for a different choice. By properly choosing $\mu$, a fixed failure rate can be guaranteed, see Section 5.5. The regions $\Omega_{\mathcal{I}^{\prime}, \boldsymbol{z}}$ are shown in Figure 5.1 for a two dimensional example. The same validation strategy, i.e., testing $\check{\boldsymbol{a}}_{\text {ILS }}$ against $\overline{\boldsymbol{a}}^{\mathcal{I}^{\prime}}$, can also be used to formulate a modified version of the ratio test and the projector test for single-subset generalized integer aperture estimation, which is not done explicitly in this dissertation.

\subsubsection{Selecting the Integer Transformation and Subset $\mathcal{I}^{\prime}$}

So far, both the transformation matrix $Z \in \mathbb{Z}^{n \times n}$ and the index set $\mathcal{I}^{\prime}$ were assumed to be a priori known. In GNSS positioning applications, they should be chosen such that a good positioning quality is obtained, as it is discussed for partial integer estimators in Section 4.4. The reasoning 
that is presented in Section 4.4 for how to choose the integer transformation also holds for singlesubset generalized integer aperture estimation. This problem is thus not further discussed. Possible criteria for selecting the subset $\mathcal{I}^{\prime}$ in $\mathfrak{I}=\left\{\emptyset, \mathcal{I}^{\prime}\right\}$ are presented in the following.

The reliability of the ambiguity estimate $\check{\boldsymbol{a}}$ can be measured with the failure rate $P_{\mathrm{F}}=$ $P\left(\mathcal{I}=\mathcal{I}^{\prime}, \check{\boldsymbol{a}} \neq \boldsymbol{a}_{\mathcal{I}^{\prime}}\right)$. By properly choosing the size and shape of the selective aperture region $\Omega_{\mathcal{I}^{\prime}}$, e.g., through a critical value $\mu$, the failure rate $P_{\mathrm{F}}$ can be guaranteed to not exceed a maximum tolerable value, independent of the strength of the underlying system model. The question is now how to select $\mathcal{I}^{\prime}$ such that a high quality of $\breve{b}$ is obtained with this fixed failure rate constraint in place. We make use of the approximate description of the precision of $\breve{b}$ for the given index set $\mathcal{I}=\mathcal{I}^{\prime}$ by means of the conditional covariance matrix $Q_{\hat{b}_{\mid} \mid \hat{\mathcal{I}}_{\mathcal{I}^{\prime}}}$, which is strictly speaking only valid if $\check{\boldsymbol{a}}$ is assumed deterministic as $\check{\boldsymbol{a}}=\boldsymbol{a}_{\mathcal{I}^{\prime}}$ with the deterministic index set $\mathcal{I}^{\prime}$, see Section 5.2. Further, we use the fixing probability $P\left(\mathcal{I}=\mathcal{I}^{\prime}\right)$. For the optimal single-subset generalized integer aperture estimator it holds that

$$
P\left(\mathcal{I}=\mathcal{I}^{\prime}\right) \geq P\left(\mathcal{I}=\mathcal{I}^{\prime \prime}\right), \quad \forall \mathcal{I}^{\prime} \subseteq \mathcal{I}^{\prime \prime}
$$

if the failure rates for both index sets are identical. This can be verified as follows. Let $\Omega_{\mathcal{I}^{\prime \prime}, \boldsymbol{z}}^{*}$ denote the optimal selective aperture decision regions for $\mathcal{I}^{\prime \prime}$ with $\mathfrak{I}=\left\{\emptyset, \mathcal{I}^{\prime \prime}\right\}$. For any $\mathcal{I}^{\prime} \subseteq \mathcal{I}^{\prime \prime}$ and $\mathfrak{I}=\left\{\emptyset, \mathcal{I}^{\prime}\right\}$, the selective aperture decision regions $\Omega_{\mathcal{I}^{\prime}, \boldsymbol{u}}^{*}$ could for instance be chosen as the union of all regions $\Omega_{\mathcal{I}^{\prime \prime}, z}^{*}$ that correspond to different integer values for the elements of $z$ that have been removed from the index set, and to identical values for the elements that are still in the set. The resulting estimator, which is not even optimal, would have a larger success rate and a smaller failure rate, while the fixing rates $P\left(\mathcal{I}=\mathcal{I}^{\prime}\right)$ and $P\left(\mathcal{I}=\mathcal{I}^{\prime \prime}\right)$ are identical. Therefore, (5.31) is valid.

We can now evaluate $\boldsymbol{Q}_{\hat{\boldsymbol{b}} \mid \hat{\boldsymbol{a}}_{\mathcal{I}^{\prime}}}$ and the fixing probability $P\left(\mathcal{I}=\mathcal{I}^{\prime}\right)$ for different choices of $\mathcal{I}^{\prime}$ and the given failure rate and based on the result decide upon which subset $\mathcal{I}^{\prime}$ to choose for $\mathfrak{I}=\left\{\emptyset, \mathcal{I}^{\prime}\right\}$. The problem with this approach is that the fixing probabilities for a given failure rate are generally difficult to compute, and even if they could be computed, it would still be a tough choice of which subset $\mathcal{I}^{\prime}$ to finally choose. The user would for instance have to define whether it is better to obtain a very high precision as given by $\boldsymbol{Q}_{\hat{b} \mid \hat{\boldsymbol{a}}_{\mathcal{I}^{\prime}}}$ with a rather small fixing rate or a comparably lower precision but with a higher fixing rate. In GNSS practice, a computationally more simple strategy would be preferable.

\subsection{Multiple-Subset Generalized Integer Aperture Estimators}

A more flexible strategy is to allow the set $\mathfrak{I}$ of possible realizations of the index set $\mathcal{I}$ of a generalized integer aperture estimator to assume more than one non-empty value. That is, the decision is not only whether or not to resolve a specific subset $\mathcal{I}^{\prime}$ of the integer parameters as discussed in Section 5.3, but there may now be truly different integer subsets for different realizations of the float solution $\hat{\boldsymbol{a}}$. Formulating an optimal generalized integer aperture estimator is now a much harder task, since this requires the joint optimization of the selective aperture decision regions $\Omega_{\mathcal{J}, \boldsymbol{z}}, \forall \mathcal{J} \in \mathfrak{I}$. Imposing a failure rate constraint $P_{\mathrm{F}}=\gamma$ still leaves infinitely many options for how to partition the pull-in regions $S_{\boldsymbol{v}}, \forall \boldsymbol{v} \in \mathbb{Z}^{n}$, into the sub-regions $\tilde{\Omega}_{\mathcal{J}, \boldsymbol{v}}$ corresponding to different index sets, cf. (5.7), which implicitly defines the probabilities $P(\mathcal{I}=\mathcal{J})$. In GNSS positioning applications we aim at obtaining a high quality of $\breve{b}$. Even with the simple approximate description of the quality of $\check{b}$ by means of the conditional covariance matrices $\boldsymbol{Q}_{\hat{\boldsymbol{b}} \mid \hat{\boldsymbol{a}}_{\mathcal{J}}}, \forall \mathcal{J} \in \mathfrak{I}$, for 
a given index set $\mathcal{I}=\mathcal{J}$ and the associated probabilities $P(\mathcal{I}=\mathcal{J})$ it is not clear what optimal means, not to mention how to actually derive the optimal estimator once an optimality criterion has been set up. Within this dissertation, no attempt is made to formulate an optimal multiple-subset generalized integer aperture estimator.

The fundamental idea of the suboptimal estimators presented in the remainder of this section is to compute a full $n$ dimensional integer solution together with a reliability measure for each scalar component. Based on these $n$ reliability measures, the index set $\mathcal{I}$ is determined and the corresponding entries of the full integer solution are accepted. In the following, three specific realizations of this strategy based on the difference test, the ratio test, and the projector test are presented and compared. Implementation aspects are discussed.

\subsubsection{Per Element Difference Test}

The difference test was introduced in Section 3.2 within the class of integer aperture estimators. It can be modified so as to be capable of selecting the subset of integer parameters to be resolved by not only testing the integer least-squares solution $\check{\boldsymbol{a}}_{\mathrm{ILS}}$ against the overall best counter hypothesis $\overline{\boldsymbol{a}}$, but against the best counter hypothesis $\overline{\boldsymbol{a}}^{i} \in \mathbb{Z}^{n}, i=1, \ldots, n$, for each scalar entry of $\check{\boldsymbol{a}}_{\mathrm{ILS}}$. The counter hypothesis of the $i$ th element is defined as the integer vector closest to $\hat{\boldsymbol{a}}$ that is different from the overall closest integer vector $\check{\boldsymbol{a}}_{\mathrm{ILS}}$ in the $i$ th component

$$
\overline{\boldsymbol{a}}^{i}=\underset{\boldsymbol{v} \in \mathbb{Z}^{n} \mid \boldsymbol{v}_{i} \neq \check{\boldsymbol{a}}_{\mathrm{ILS}, i}}{\operatorname{argmin}}\|\hat{\boldsymbol{a}}-\boldsymbol{v}\|_{\boldsymbol{Q}_{\hat{\boldsymbol{a}}}}^{2} .
$$

At least one of the counter hypotheses $\overline{\boldsymbol{a}}^{i}$ is identical to the overall best counter hypothesis $\overline{\boldsymbol{a}}$, see (3.31). The index set $\mathcal{I}$ of the integer parameters to be resolved follows by evaluating the difference test for each of these $n$ counter hypothesis as

$$
\mathcal{I}=\left\{i=1, \ldots, n \mid\left\|\hat{\boldsymbol{a}}-\overline{\boldsymbol{a}}^{i}\right\|_{\boldsymbol{Q}_{\hat{\boldsymbol{a}}}}^{2}-\left\|\hat{\boldsymbol{a}}-\check{\boldsymbol{a}}_{\mathrm{ILS}}\right\|_{\boldsymbol{Q}_{\hat{\boldsymbol{a}}}}^{2} \geq \mu\right\}
$$

with a single critical value $\mu$. The entries $\boldsymbol{\Gamma}_{\mathcal{I}} \check{\boldsymbol{a}}_{\mathrm{ILS}}$ of the integer least-squares solution corresponding to the resulting index set $\mathcal{I}$ are accepted as an integer estimate for $\boldsymbol{a}_{\mathcal{I}}$. With this strategy, the set of possible index sets $\mathfrak{I}$ can generally contain all $2^{n}$ possible realizations. This combined subset selection, estimation, and validation strategy is introduced in Brack and Günther (2014) and is a generalized integer aperture estimator defined by the selective aperture decision regions

$$
\begin{aligned}
& \tilde{\Omega}_{\mathcal{J}, \boldsymbol{v}}=\{\boldsymbol{x} \in S_{\boldsymbol{v}}\left|\forall i \in \mathcal{J}:\|\boldsymbol{x}-\boldsymbol{u}\|_{\boldsymbol{Q}_{\hat{a}}}^{2}-\|\boldsymbol{x}-\boldsymbol{v}\|_{\boldsymbol{Q}_{\hat{a}}}^{2} \geq \mu, \forall \boldsymbol{u} \in \mathbb{Z}^{n}\right| \boldsymbol{u}_{i} \neq \boldsymbol{v}_{i}, \\
&\left.\forall i \in \overline{\mathcal{J}}: \exists \boldsymbol{u} \in \mathbb{Z}^{n} \mid \boldsymbol{u}_{i} \neq \boldsymbol{v}_{i},\|\boldsymbol{x}-\boldsymbol{u}\|_{\boldsymbol{Q}_{\hat{a}}}^{2}-\|\boldsymbol{x}-\boldsymbol{v}\|_{\boldsymbol{Q}_{\hat{a}}}^{2}<\mu\right\}, \quad \forall \boldsymbol{v} \in \mathbb{Z}^{n},
\end{aligned}
$$

for each of the $2^{n}$ possible index sets $\mathcal{J}$, where not all regions $\tilde{\Omega}_{\mathcal{J}, \boldsymbol{v}}$ have to be non-empty. Thereby, the set $\overline{\mathcal{J}}$ is defined such that $\mathcal{J} \cup \overline{\mathcal{J}}=\{1, \ldots, n\}$, and $S_{\boldsymbol{v}}$ are the pull-in regions of integer leastsquares. The index set $\mathcal{I}$ is defined uniquely in (5.33) for any float solution $\hat{\boldsymbol{a}} \in \mathbb{R}^{n}$, which means that the first two conditions in (5.6) are fulfilled. Due to the regularity of the integer grid, a shift of the float solution by an arbitrary integer vector $\boldsymbol{u} \in \mathbb{Z}^{n}$ causes a shift of the integer least-squares solution $\check{\boldsymbol{a}}_{\mathrm{ILS}}$ and all $n$ counter hypotheses $\overline{\boldsymbol{a}}^{i}$ by the same integer, i.e., the test values in (5.33) remain unchanged and the third condition in (5.6) is also met. The per element difference test is therefore a valid generalized integer aperture estimator. 


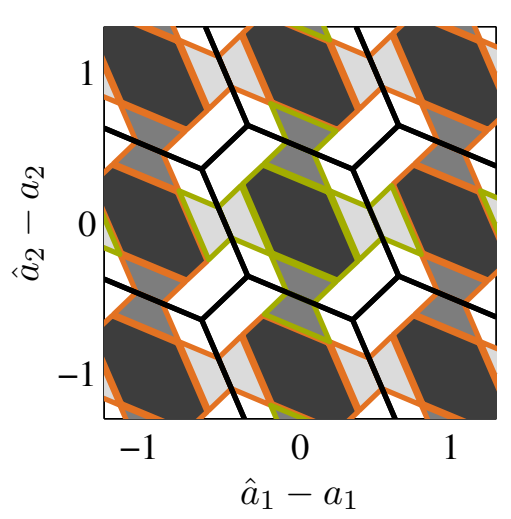

(a) Per element difference test

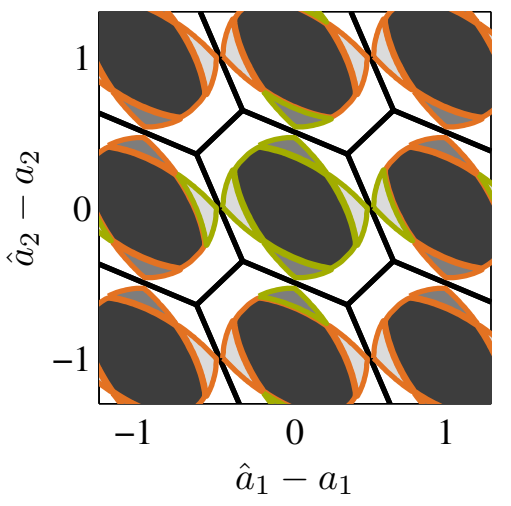

(b) Per element ratio test

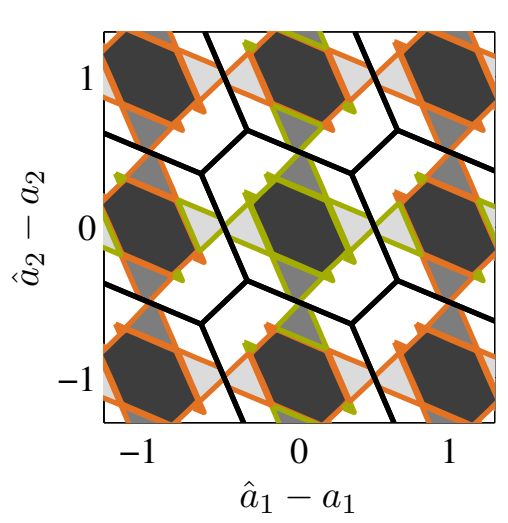

(c) Per element projector test

Figure 5.3: The selective aperture decision regions $\tilde{\Omega}_{\mathcal{J}, \boldsymbol{v}}$ are shown for $\mathcal{J}=\{1,2\}, \mathcal{J}=\{1\}, \mathcal{J}=\{2\}$, and $\mathcal{J}=\emptyset$ from dark gray to white. Green and orange edges indicate correct and incorrect integer estimates, respectively. The boundaries of the pull-in regions $S_{\boldsymbol{v}}$ of the underlying integer estimator are drawn in black. This figure is the equivalence of Figure 3.4 for generalized integer aperture estimation.

The integer least-squares solution $\check{\boldsymbol{a}}_{\mathrm{ILS}}$ can be equivalently computed for any integer transformation $\boldsymbol{Z}$, but this does not hold for the counter hypotheses $\overline{\boldsymbol{a}}^{i}$. A more detailed discussion about the implementation of the above scheme is given in Section 5.4.4. By properly choosing the critical value $\mu$, a fixed failure rate $P_{\mathrm{F}}$ can be guaranteed, see Section 5.5. It is noted that this strategy is very similar to the difference test based single-subset generalized integer aperture estimator from Section 5.3.2, since it essentially combines the single-subset selective aperture regions following from (5.30) for all $2^{n}-1$ non-empty subsets, using the union of all involved index sets $\mathcal{J}$ whenever multiple single-subset selective aperture regions intersect. The regions $\tilde{\Omega}_{\mathcal{J}, v}$ corresponding to the four index sets $\mathcal{J}$ in a two dimensional example are shown in Figure 5.3. The selective aperture region $\Omega_{\{1,2\}}$ is identical to the aperture region of the conventional difference test. Since the integer mapping is defined through selecting entries of the integer least-squares solution $\check{\boldsymbol{a}}_{\mathrm{ILS}}$, the region that leads to correct integer estimates also contains parts of the pull-in regions associated to integers other than $\boldsymbol{a}$, but with only the first or second component being identical to $a_{1}$ or $a_{2}$, respectively.

An idea that is similar to the per element difference test is soft-output detection or decoding in communications, which not only provides a hard estimate in form of the maximum likelihood solution-which in our case is the integer least-squares solution $\check{\boldsymbol{a}}_{\mathrm{ILS}}$-but also some information about the reliability of each element. A popular reliability measure used in iterative detection/decoding algorithms in communication systems are log-likelihood ratios (LLRs) (Hagenauer et al. 1996). With the Gaussian distribution of the float solution $\hat{a}$, the likelihood function for estimating $\boldsymbol{a}$ from $\hat{\boldsymbol{a}}$ is given by $L(\hat{\boldsymbol{a}} ; \boldsymbol{v}) \propto \exp \left(-\frac{1}{2}\|\hat{\boldsymbol{a}}-\boldsymbol{v}\|_{\boldsymbol{Q}_{\hat{a}}}^{2}\right), \boldsymbol{v} \in \mathbb{Z}^{n}$. Evaluating the LLR between the integer least-squares solution $\check{\boldsymbol{a}}_{\mathrm{ILS}}$ and the best counter hypothesis $\overline{\boldsymbol{a}}^{i}$ for the $i$ th component as defined in (5.32) results in

$$
\log \frac{L\left(\hat{\boldsymbol{a}} ; \check{\boldsymbol{a}}_{\mathrm{ILS}}\right)}{L\left(\hat{\boldsymbol{a}} ; \overline{\boldsymbol{a}}^{i}\right)}=\frac{1}{2}\left\|\hat{\boldsymbol{a}}-\overline{\boldsymbol{a}}^{i}\right\|_{\boldsymbol{Q}_{\hat{\boldsymbol{a}}}}^{2}-\frac{1}{2}\left\|\hat{\boldsymbol{a}}-\check{\boldsymbol{a}}_{\mathrm{ILS}}\right\|_{\boldsymbol{Q}_{\hat{a}}}^{2},
$$

which is simply a scaled version of the difference test for the $i$ th element. Instead of passing this reliability measure on to a subsequent detection or decoding instance, we use it to decide about whether or not to include the index $i$ in the set $\mathcal{I}$. 


\subsubsection{Per Element Ratio Test}

The same strategy of testing each of the entries of the integer least-squares solution $\check{\boldsymbol{a}}_{\mathrm{ILS}}$ against the best counter hypothesis $\overline{\boldsymbol{a}}^{i}$ can be used with the ratio test instead of the difference test. The entries $\boldsymbol{\Gamma}_{\mathcal{I}} \check{\boldsymbol{a}}_{\mathrm{ILS}}$ of the integer least-squares solution are accepted as an integer estimate for $\boldsymbol{a}_{\mathcal{I}}$, where the index set $\mathcal{I}$ is now determined as

$$
\mathcal{I}=\left\{i=1, \ldots, n \mid\left\|\hat{\boldsymbol{a}}-\check{\boldsymbol{a}}_{\mathrm{ILS}}\right\|_{\boldsymbol{Q}_{\hat{\boldsymbol{a}}}}^{2} \leq \mu\left\|\hat{\boldsymbol{a}}-\overline{\boldsymbol{a}}^{i}\right\|_{\boldsymbol{Q}_{\hat{\boldsymbol{a}}}}^{2}\right\}
$$

with a single critical value $\mu$. All $2^{n}$ possible realizations of $\mathcal{I}$ can generally be obtained. This combined subset selection, estimation, and validation strategy is introduced in Brack and Günther (2014) and is a generalized integer aperture estimator defined by the selective aperture decision regions

$$
\begin{aligned}
\tilde{\Omega}_{\mathcal{J}, \boldsymbol{v}}=\left\{\boldsymbol{x} \in S_{\boldsymbol{v}}\left|\forall i \in \mathcal{J}:\|\boldsymbol{x}-\boldsymbol{v}\|_{\boldsymbol{Q}_{\hat{\boldsymbol{a}}}}^{2} \leq \mu\|\boldsymbol{x}-\boldsymbol{u}\|_{\boldsymbol{Q}_{\hat{a}}}^{2}, \forall \boldsymbol{u} \in \mathbb{Z}^{n}\right| \boldsymbol{u}_{i} \neq \boldsymbol{v}_{i},\right. \\
\left.\forall i \in \overline{\mathcal{J}}: \exists \boldsymbol{u} \in \mathbb{Z}^{n} \mid \boldsymbol{u}_{i} \neq \boldsymbol{v}_{i},\|\boldsymbol{x}-\boldsymbol{v}\|_{\boldsymbol{Q}_{\hat{a}}}^{2}>\mu\|\boldsymbol{x}-\boldsymbol{u}\|_{\boldsymbol{Q}_{\hat{a}}}^{2}\right\}, \quad \forall \boldsymbol{v} \in \mathbb{Z}^{n}
\end{aligned}
$$

for each of the $2^{n}$ index sets $\mathcal{J}$, where $S_{\boldsymbol{v}}$ are the pull-in regions of integer least-squares and $\overline{\mathcal{J}}$ is defined such that $\mathcal{J} \cup \overline{\mathcal{J}}=\{1, \ldots, n\}$. Following the same argumentation as for the per element difference test in Section 5.4.1 shows that the per element ratio test is a valid generalized integer aperture estimator. By properly choosing the critical value $\mu$, a fixed failure rate $P_{\mathrm{F}}$ can be guaranteed, see Section 5.5. The regions $\tilde{\Omega}_{\mathcal{J}, \boldsymbol{v}}$ corresponding to the four index sets $\mathcal{J}$ in a two dimensional example are shown in Figure 5.3. The selective aperture region $\Omega_{\{1,2\}}$ is identical to the aperture region of the conventional ratio test.

\subsubsection{Per Element Projector Test}

The third acceptance test that is discussed for the strategy of comparing the integer least-squares solution $\check{\boldsymbol{a}}_{\text {ILS }}$ against the best counter hypothesis $\overline{\boldsymbol{a}}^{i}$ for each element is the projector test. The set of indexes $\mathcal{I}$, for which the corresponding entries $\boldsymbol{\Gamma}_{\mathcal{I}} \check{\boldsymbol{a}}_{\mathrm{ILS}}$ of the integer least-squares solution are accepted as an integer solution, is given by

$$
\mathcal{I}=\left\{i=1, \ldots, n \mid\left(\overline{\boldsymbol{a}}^{i}-\check{\boldsymbol{a}}_{\mathrm{ILS}}\right)^{\mathrm{T}} \boldsymbol{Q}_{\hat{\boldsymbol{a}}}^{-1}\left(\hat{\boldsymbol{a}}-\check{\boldsymbol{a}}_{\mathrm{ILS}}\right) \leq \mu\left\|\overline{\boldsymbol{a}}^{i}-\check{\boldsymbol{a}}_{\mathrm{ILS}}\right\|_{\boldsymbol{Q}_{\hat{\boldsymbol{a}}}}\right\}
$$

with the single critical value $\mu$. All $2^{n}$ possible realizations of $\mathcal{I}$ can generally be obtained. This combined subset selection, estimation, and validation strategy is a generalized integer aperture estimator defined by the selective aperture decision regions

$$
\begin{gathered}
\tilde{\Omega}_{\mathcal{J}, \boldsymbol{v}}=\left\{\boldsymbol{x} \in S_{\boldsymbol{v}} \mid \forall i \in \mathcal{J}:(\boldsymbol{u}-\boldsymbol{v})^{\mathrm{T}} \boldsymbol{Q}_{\hat{\boldsymbol{a}}}^{-1}(\boldsymbol{x}-\boldsymbol{v}) \leq \mu\|\boldsymbol{x}-\boldsymbol{v}\|_{\boldsymbol{Q}_{\hat{a}}}, \boldsymbol{u}=\underset{\boldsymbol{w} \in \mathbb{Z}^{n} \mid \boldsymbol{w}_{i} \neq \boldsymbol{v}_{i}}{\operatorname{argmin}}\|\boldsymbol{x}-\boldsymbol{w}\|_{\boldsymbol{Q}_{\hat{a}}}^{2},\right. \\
\left.\forall i \in \overline{\mathcal{J}}:(\boldsymbol{u}-\boldsymbol{v})^{\mathrm{T}} \boldsymbol{Q}_{\hat{\boldsymbol{a}}}^{-1}(\boldsymbol{x}-\boldsymbol{v})>\mu\|\boldsymbol{x}-\boldsymbol{v}\|_{\boldsymbol{Q}_{\hat{a}}}, \boldsymbol{u}=\underset{\boldsymbol{w} \in \mathbb{Z}^{n} \mid \boldsymbol{w}_{i} \neq \boldsymbol{v}_{i}}{\operatorname{argmin}}\|\boldsymbol{x}-\boldsymbol{w}\|_{\boldsymbol{Q}_{\hat{a}}}^{2}\right\},
\end{gathered}
$$

for each of the $2^{n}$ index sets $\mathcal{J}$, where $S_{\boldsymbol{v}}$ are the pull-in regions of integer least-squares and $\overline{\mathcal{J}}$ is defined such that $\mathcal{J} \cup \overline{\mathcal{J}}=\{1, \ldots, n\}$. The same argumentation as for the per element difference test in Section 5.4.1 shows that the per element projector test is a valid generalized integer aperture 
estimator. By properly choosing the critical value $\mu$, a fixed failure rate $P_{\mathrm{F}}$ can be guaranteed, see Section 5.5. The regions $\tilde{\Omega}_{\mathcal{J}, v}$ corresponding to the four index sets $\mathcal{J}$ in a two dimensional example are shown in Figure 5.3. The selective aperture region $\Omega_{\{1,2\}}$ is not identical to the aperture region of the projector test.

\subsubsection{Computational Aspects}

The underlying partial integer estimator defining the integer solutions for the $2^{n}-1$ non-empty index sets for the per element acceptance tests discussed in the previous sections is full integer least-squares followed by a selection of entries, see Section 4.3.2. This enables a very efficient implementation of these estimators, since only a single integer solution-the full integer least-squares solution-is required, independent of what the subset $\mathcal{I}$ turns out to be. Otherwise, one would have to (iteratively) compute multiple integer estimates for different subsets and test them for acceptance, cf. Verhagen et al. (2011) or Parkins (2011). The solution of the integer least-squares problem by means of a tree search formulation is discussed in Section 3.1.4. The present situation is, however, slightly different, since not only the integer least-squares solution $\check{\boldsymbol{a}}_{\mathrm{ILS}}$ is required, but also the $n$ counter hypotheses $\overline{\boldsymbol{a}}^{i}$ in order to evaluate the acceptance tests. Two strategies for the computation of these $n+1$ integer vectors using a tree search formulation are presented in the following.

The first and probably more intuitive approach is to perform a repeated tree search. That is, in a first search run, the integer least-squares solution $\check{\boldsymbol{a}}_{\mathrm{ILS}}$ is determined. After that, $n$ additional search runs are performed on the same search tree to determine the counter hypotheses $\overline{\boldsymbol{a}}^{i}, i=1, \ldots, n$. According to (5.32), they are the integer vectors with the smallest weighted squared distance to $\hat{\boldsymbol{a}}$ for which the $i$ th component is different from the $i$ th component of $\check{\boldsymbol{a}}_{\text {ILS }}$. This restriction is equivalent to blocking all branches of the search tree that correspond to an integer $z \in \mathbb{Z}^{n}$ with $z_{i}=\check{a}_{\mathrm{ILS}, i}$. Computing $\overline{\boldsymbol{a}}^{i}$ now means to find the best integer candidate in this reduced search tree.

Obviously, the $n+1$ search runs for this repeated tree search imply a high computational burden. It is therefore desirable to find a more efficient tree traversal strategy, preferably one for which only a single search run is required so that each node of the tree is at most visited once. The main idea to accomplish such a single tree search solution is to keep a list during the search process that contains the best integer vector that was found so far, i.e., the current candidate for the integer least-squares solution, and the corresponding candidates for the counter hypotheses. Let $\check{z}_{\mathrm{ILS}} \in \mathbb{Z}^{n}$ and $\overline{\boldsymbol{z}}^{i} \in \mathbb{Z}^{n}, i=1, \ldots, n$, denote the current candidates for the integer least-squares solution and the counter hypotheses. Whenever a leaf of the search tree associated to an integer $\boldsymbol{z} \in \mathbb{Z}^{n}$ with weighted squared distance $d(\boldsymbol{z})$, cf. Section 3.1.4, is reached, the following two cases have to be distinguished:

1) $d(\boldsymbol{z})<d\left(\check{\boldsymbol{z}}_{\mathrm{ILS}}\right)$ : The integer $\boldsymbol{z}$ is the new candidate for the integer least-squares solution. The updates in the candidate list are $\overline{\boldsymbol{z}}^{i} \leftarrow \check{\boldsymbol{z}}_{\mathrm{ILS}}, \forall i=1, \ldots, n \mid \check{z}_{\mathrm{ILS}, i} \neq z_{i}$, and $\check{z}_{\mathrm{ILS}} \leftarrow \boldsymbol{z}$. That is, for each entry of $\check{z}_{\text {ILS }}$ that is changed during the update process, the previous $\check{z}_{\text {ILS }}$ is the new candidate for the counter hypothesis.

2) $d(\boldsymbol{z}) \geq d\left(\check{\boldsymbol{z}}_{\mathrm{ILS}}\right)$ : Only the counter hypotheses have to be checked. The updates in the candidate list are $\overline{\boldsymbol{z}}^{i} \leftarrow \boldsymbol{z}, \forall i=1, \ldots, n \mid \check{z}_{\mathrm{ILS}, i} \neq z_{i}$ and $d(\boldsymbol{z})<d\left(\overline{\boldsymbol{z}}^{i}\right)$.

The weighted squared distances $d\left(\overline{\boldsymbol{z}}^{i}\right), i=1, \ldots, n$, and $d\left(\check{\boldsymbol{z}}_{\mathrm{ILS}}\right)$ are initialized with infinity. A similar strategy has been developed for the detection problem in soft-decision multiple-input 
multiple-output (MIMO) communication systems in Jaldén and Ottersten (2005), Studer et al. (2008), and Studer and Bölcskei (2010).

In order to keep the necessary computational effort at a minimum for either of the search strategies, the selection of the search radius $\lambda$ around a given float solution $\hat{\boldsymbol{a}}$ that is used to remove irrelevant parts of the search tree is critical. Similarly to the integer least-squares problem, we can make use of the easily computable integer bootstrapping estimator to find a value for $\lambda^{2}$ such that $\check{\boldsymbol{a}}_{\text {ILS }}$ as well as the $n$ counter hypotheses $\overline{\boldsymbol{a}}^{i}$ are contained in the search ellipsoid. The components of the integer bootstrapping estimate $\check{\boldsymbol{a}}_{\mathrm{IB}}$ are given by $\check{a}_{\mathrm{IB}, i}=\left\lfloor\hat{a}_{i \mid I}\right\rceil$, see (3.9). This sequential integer fixing scheme is now repeated $n$ times, where in the $i$ th iteration the $i$ th element is chosen different from the integer bootstrapping solution as the second closest scalar integer. The $i$ th component of this integer bootstrapping based counter hypothesis $\overline{\boldsymbol{a}}_{\mathrm{IB}}^{i}$ is therefore given by

$$
\bar{a}_{\mathrm{IB}, i}^{i}=\left\lfloor\hat{a}_{i \mid I}\right\rceil+\operatorname{sign}\left(\hat{a}_{i \mid I}-\left\lfloor\hat{a}_{i \mid I}\right\rceil\right) .
$$

All other components of $\overline{\boldsymbol{a}}_{\mathrm{IB}}^{i}$ are computed identically to the integer bootstrapping solution. Choosing the size parameter $\lambda^{2}$ of the search space as

$$
\lambda^{2}=\max \left\{\left\|\hat{\boldsymbol{a}}-\check{\boldsymbol{a}}_{\mathrm{IB}}\right\|_{\boldsymbol{Q}_{\hat{\boldsymbol{a}}}}^{2},\left\|\hat{\boldsymbol{a}}-\overline{\boldsymbol{a}}_{\mathrm{IB}}^{1}\right\|_{\boldsymbol{Q}_{\hat{\boldsymbol{a}}}}^{2}, \ldots,\left\|\hat{\boldsymbol{a}}-\overline{\boldsymbol{a}}_{\mathrm{IB}}^{n}\right\|_{\boldsymbol{Q}_{\hat{\boldsymbol{a}}}}^{2}\right\}
$$

guarantees that by examining all integer vectors within a radius of $\lambda$ from $\hat{\boldsymbol{a}}$, at least two different values are obtained for each scalar element. In other words, there is a valid counter hypothesis to the integer least-squares solution $\check{\boldsymbol{a}}_{\mathrm{ILS}}$ for each element and the acceptance tests in (5.33), (5.36), and (5.38) can be evaluated. This method can be refined by removing the largest elements in the set of (5.41), given that the integer vectors corresponding to the remaining elements still assume at least two different values for each scalar component.

Much smaller values for $\lambda^{2}$ leading to much shorter search times may be possible when we think about the purpose of the counter hypotheses. The integer values that are assumed by them are per se completely irrelevant, since the value of the integer estimate is fully determined by selecting entries of $\check{\boldsymbol{a}}_{\mathrm{ILS}}$. The only relevant question is, whether or not the acceptance tests based on $\check{\boldsymbol{a}}_{\mathrm{ILS}}$ and $\overline{\boldsymbol{a}}^{i}$ exceed the critical value $\mu$ or not. The index $i$ of all components for which no valid counter hypothesis $\overline{\boldsymbol{a}}^{i}$ exists within the search spaces with squared radii

$$
\lambda^{2}=\left\|\hat{\boldsymbol{a}}-\check{\boldsymbol{a}}_{\mathrm{ILS}}\right\|_{\boldsymbol{Q}_{\hat{\boldsymbol{a}}}}^{2}+\mu \quad \text { and } \quad \lambda^{2}=\frac{\left\|\hat{\boldsymbol{a}}-\check{\boldsymbol{a}}_{\mathrm{ILS}}\right\|_{\boldsymbol{Q}_{\hat{\boldsymbol{a}}}}^{2}}{\mu}
$$

around $\hat{\boldsymbol{a}}$ for the per element difference test (left) and ratio test (right), respectively, are automatically part of the index set $\mathcal{I}$. Unfortunately, this strategy cannot be easily applied to the per element projector test. The unknown $\left\|\hat{\boldsymbol{a}}-\check{\boldsymbol{a}}_{\mathrm{ILS}}\right\|_{\boldsymbol{Q}_{\hat{\boldsymbol{a}}}}^{2}$ can be upper bounded by $\left\|\hat{\boldsymbol{a}}-\check{\boldsymbol{a}}_{\mathrm{IB}}\right\|_{\boldsymbol{Q}_{\hat{\boldsymbol{a}}}}^{2}$. The radius of the search space can optionally be reduced whenever a new candidate for $\check{\boldsymbol{a}}_{\mathrm{ILS}}$ is found or as soon as a valid counter hypothesis $\overline{\boldsymbol{a}}^{i}, \forall i=1, \ldots, n$, has been found.

If the transformation matrix $Z$ in (2.82) is chosen other than a decorrelation matrix, the search can still be performed at low complexity in a decorrelated space after an additional integer transformation with the single tree search approach. This additional transformation just has to be inverted whenever an integer candidate $z$ is found before editing the list of candidates for the counter hypotheses. One could of course also think of other strategies than the ones introduced above. A possible variant is to search for all integer vectors within the search space with radius $\lambda$ and afterwards select those elements thereof that correspond to $\check{\boldsymbol{a}}_{\mathrm{ILS}}$ and $\overline{\boldsymbol{a}}^{i}, i=1, \ldots, n$. This approach 
Table 5.1: GNSS system parameters used for analysis of per element acceptance tests.

\begin{tabular}{ll}
\hline Parameter & Considered values \\
\hline Systems & GPS, Galileo, combined GPS+Galileo \\
Signals & L1, L1+L2, E1, E1+E5a, L1+E1, L1+L2+E1+E5a \\
$\quad$ code std., zenith & L1, L2, E1: 25 cm, E5a: $20 \mathrm{~cm}$ \\
$\quad$ carrier-phase std., zenith & $3 \mathrm{~mm}$ for all signals \\
Noise scaling & $0.5,1.0,2.0$ \\
Elevation cutoff angle & $10^{\circ}$ \\
Receiver positions & $30^{\circ} \mathrm{S}, 0^{\circ} \mathrm{N}, 30^{\circ} \mathrm{N}, 60^{\circ} \mathrm{N}$ \\
Time instances & 100 different epochs in 7 day period \\
Between rec. ionosph. std., zenith & $0 \mathrm{~cm}, 0.71 \mathrm{~cm}, 1.41 \mathrm{~cm}, 3.54 \mathrm{~cm}, 7.07 \mathrm{~cm}$ \\
\hline
\end{tabular}

requires additional searches within the set of integer vectors, which might, however, be of negligible complexity.

The per element difference test and ratio test can be easily integrated in the implementation of the popular LAMBDA method. For a given critical value $\mu$, the radius of the search space follows from (5.42). The search algorithm of the LAMBDA method (de Jonge and Tiberius 1996) is then modified so as to not store a list of the best integer candidates, but rather a list of the candidates $\check{\boldsymbol{z}}_{\mathrm{ILS}}$ and $\overline{\boldsymbol{z}}^{i}$ as described above. The final subset $\mathcal{I}$ and ambiguity estimates $\check{\boldsymbol{a}}$ can then easily be determined.

All implementation details that are discussed in this section can-with small modifications-also be applied to the difference test based single-subset estimator in Section 5.3.2.

\subsubsection{Selecting the Integer Transformation}

For the per element acceptance tests it is not required to a priori define the set of possible index sets $\mathfrak{I}$ like for the single subset schemes presented in Section 5.3. All $2^{n}$ realizations of $\mathcal{I}$ can generally be obtained, and the choice is implicitly defined through the single aperture parameter $\mu$.

What has to be defined by the user is the parameterization of the float solution $\hat{\boldsymbol{a}}$ via the transformation matrix $Z$ in (2.82). Following the discussion for partial integer estimators in Section 4.4, choosing $Z$ as a decorrelation transformation is very useful for partial ambiguity resolution in GNSS positioning applications. This is easily demonstrated for the per element acceptance tests as presented above. For the usually highly correlated original double difference integer ambiguities, the overall best counter hypothesis $\overline{\boldsymbol{a}}$ is very likely to be different from $\check{\boldsymbol{a}}_{\mathrm{ILS}}$ in many or even all entries, which leads to identical counter hypotheses $\overline{\boldsymbol{a}}^{i}$ for many elements $i$. Identical counter hypotheses for all elements, however, mean that $\mathcal{I}$ is either the full set or the empty set, since the acceptance test is then passed for all elements or for none. A true advantage of partial ambiguity resolution can therefore only be expected when the counter hypotheses are likely to be different, i.e., in a decorrelated ambiguity space.

\subsubsection{Comparison and Performance Evaluation}

In a simulation based numerical study, the performance of the per element difference test, ratio test, and projector test is investigated. The main goals are to examine whether it is possible to make a statement about which of these estimators to prefer, and to identify possible advantages compared 
to the conventional difference test, ratio test, and projector test for estimating the full set of integer parameters. The system models that are used are single baseline single and combined GPS or/and Galileo positioning examples. The GPS constellation is modeled as seen during GPS week 1,783, and the Galileo system is simulated as Walker Delta $56^{\circ}: 27 / 3 / 1$ constellation (Walker 1984). In the dual GNSS case, a separate pivot satellite is chosen for each system. The parameter settings for the observation model are listed in Table 5.1, where the measurement and ionospheric standard deviations are used with the elevation dependent exponential weighting function as explained in Section 2.3, and the measurement standard deviations are multiplied with different scaling factors in order to account for receivers of different quality. All possible combinations of the presented values are considered. Differential tropospheric delays are assumed absent.

A first performance measure that is computed is the probability $P_{\text {fix }}=P_{\mathrm{S}}+P_{\mathrm{F}}$ of resolving any integer parameters at all for a given failure rate constraint $P_{\mathrm{F}}=\gamma$. The corresponding fixed failure rate critical values $\mu$ are computed via Monte Carlo integration for each system model and acceptance test, see Section 5.5. The three per element acceptance tests are applied in the LAMBDA decorrelated space (Teunissen 1995b). The resulting fixing probabilities for a fixed failure rate of $P_{\mathrm{F}}=0.1 \%$ are shown in blue as a function of the integer least-squares failure rate $P_{\mathrm{F}, \mathrm{ILS}}$ in Figure 5.4 separately for different available signals. Each data point corresponds to a different positioning case. The fixing probabilities of the conventional acceptance tests, for which either the full set of integer parameters is resolved or none of them is, see Section 3.2, are shown in green. With decreasing model strength, i.e., with increasing integer least-squares failure rate $P_{\mathrm{F}, \mathrm{ILS}}$, the fixing probabilities $P_{\text {fix }}$ of the conventional acceptance tests decrease very fast for all three tests. With the per element tests, which allow for resolving subsets of the integer parameters, the fixing probabilities $P_{\text {fix }}$ are clearly larger for the same failure rate. The highest fixing probabilities are obtained with the per element difference test. For the dual frequency combined GPS+Galileo cases, it is practically always possible to resolve at least one integer parameter. The performance of the per element ratio test is slightly worse compared to the difference test, yet still comparable, but the per element projector test leads to clearly smaller fixing probabilities.

It is of course not fair to judge the performance of the estimators based only on the fixing probability $P_{\text {fix }}=P_{\mathrm{S}}+P_{\mathrm{F}}$, since the event success can mean very different things. For the conventional acceptance tests, success means to correctly resolve the full set of integer parameters, whereas for the per element tests it means to correctly resolve at least one integer parameter, given that no other integer parameter is resolved wrongly, which is a much weaker statement. Similarly, the fixing probabilities of the three per element tests cannot be simply compared, since the corresponding random index sets $\mathcal{I}$ have different properties. A statement that can be made so far is that the results show that the per element tests can be very useful in the sense of dealing with situations that do not allow for a reliable estimation of the full set of integer parameters.

A second performance measure that is investigated is the average ratio of resolved integer parameters $n_{\text {fixed }} / n$ for a given failure rate constraint $P_{\mathrm{F}}=\gamma$, with $n_{\text {fixed }}=\mathrm{E}[|\mathcal{I}|]$. The resulting ratios $n_{\text {fixed }} / n$ of the three per element acceptance tests are computed via Monte Carlo integration and shown in blue as a function of the integer least-squares failure rate $P_{\mathrm{F}, \mathrm{ILS}}$ in Figure 5.5 for a fixed failure rate of $P_{\mathrm{F}}=0.1 \%$. The results of the per element difference test and ratio test are comparable with small advantages for the former, but the per element projector test on average resolves clearly fewer integer parameters for the given failure rate. For the dual frequency combined GPS+Galileo cases, the average ratio of resolved integer parameters is mostly over $80 \%$ with the per element difference test. Combined with the fixing probability of practically $100 \%$ from Figure 5.4, this scheme should therefore be very useful for GNSS positioning applications. 

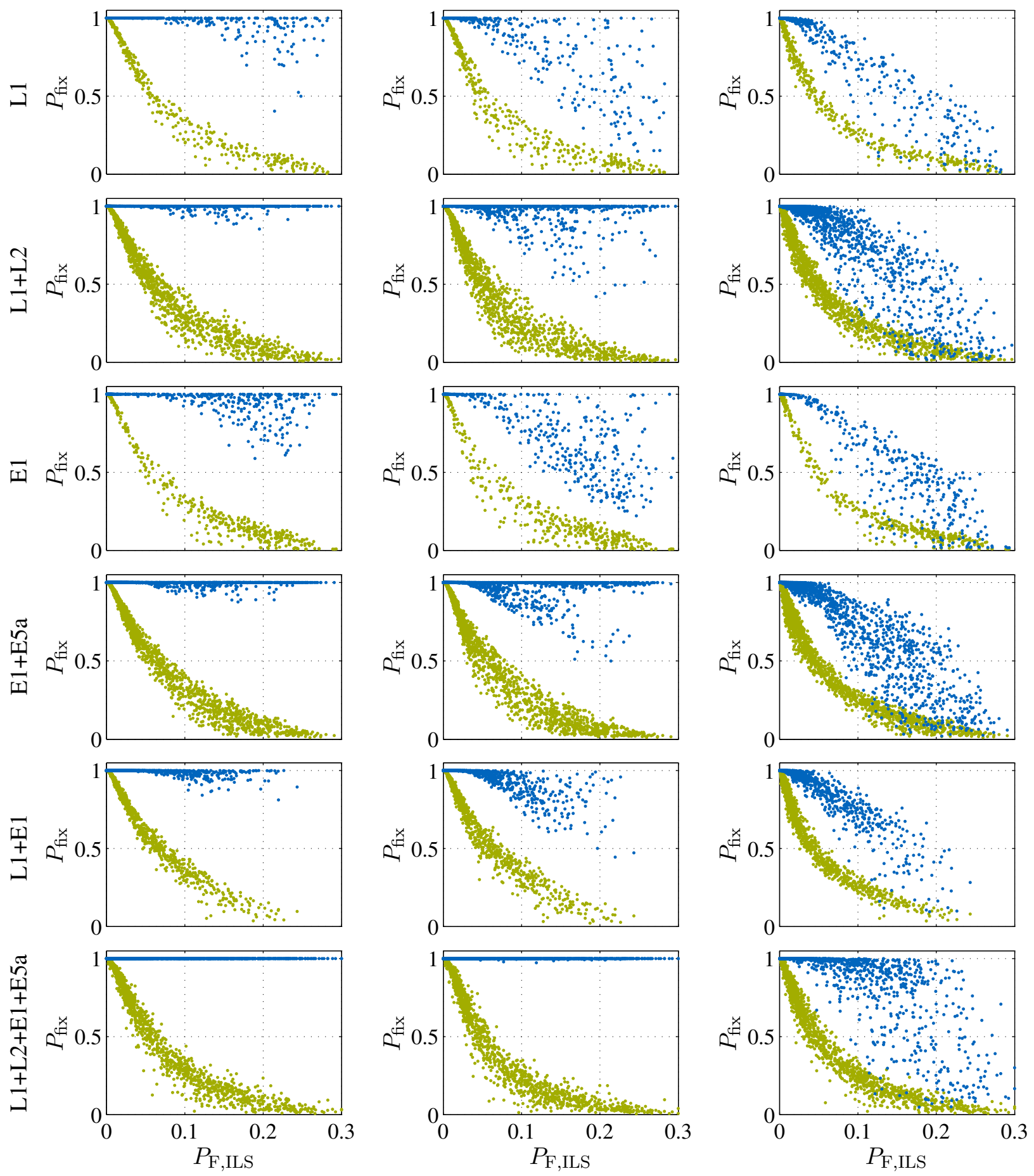

(a) (Per element) difference test

(b) (Per element) ratio test

(c) (Per element) projector test

Figure 5.4: Fixing probability $P_{\text {fix }}$ of per element acceptance tests (blue) and conventional acceptance tests (green) against the integer least-squares failure rate $P_{\mathrm{F}, \mathrm{ILS}}$ for various GNSS positioning models and a fixed failure rate of $P_{\mathrm{F}}=0.1 \%$; columns correspond to different tests, rows to different available signals. 

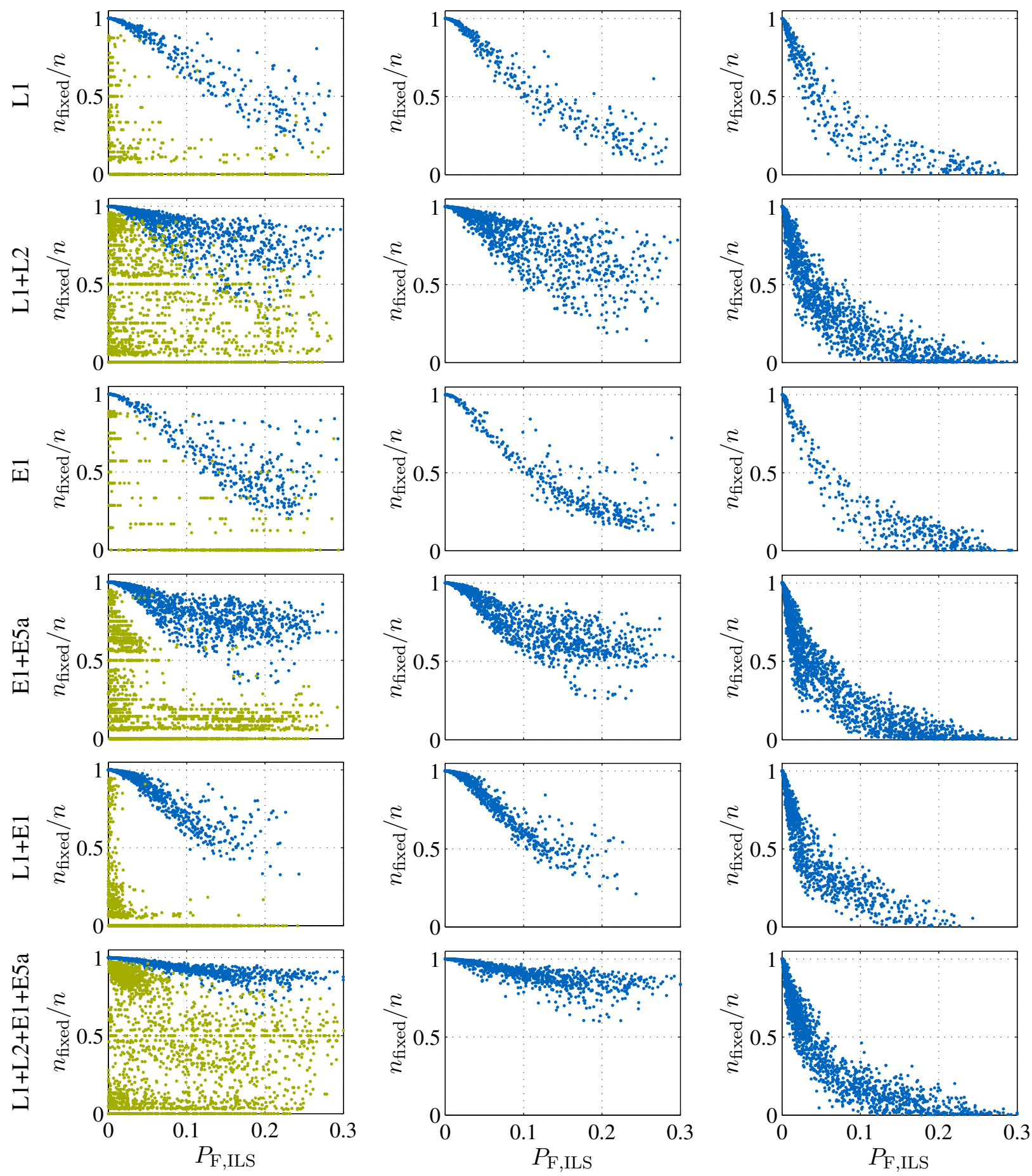

(a) Per element difference test

(b) Per element ratio test

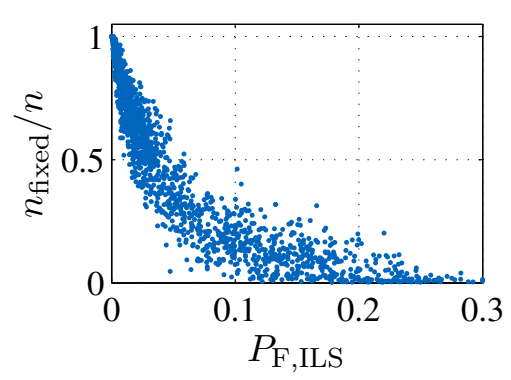

(c) Per element projector test

Figure 5.5: Ratio of fixed ambiguities $n_{\text {fixed }} / n$ of per element acceptance tests (blue) and truncated integer bootstrapping (green) against integer least-squares failure rate $P_{\mathrm{F}, \mathrm{ILS}}$ for various GNSS positioning models and a fixed failure rate of $P_{\mathrm{F}}=0.1 \%$; columns correspond to different tests, rows to different available signals. 
The green data points in the first column represent the ratio of resolved integer parameters for a model-driven partial integer estimation scheme, where the subset of integer parameters to be resolved is determined only based on the underlying system model using the failure rate of truncated integer bootstrapping, see (4.32). The same failure rate constraint and decorrelation method are used for this subset selection scheme. The results show that this model-driven scheme generally resolves a much smaller number of integer parameters, quite often none at all, even for very strong system models with very small $P_{\mathrm{F}, \mathrm{ILS}}$. The average ratio of resolved integer parameters for the conventional acceptance tests are identical to the values of the fixing probability. Comparing Figure 5.4 to Figure 5.5 shows that-at least for the difference test and ratio test-the per element tests on average thus also resolve more integer parameters than the conventional acceptance tests.

To summarize, the per element difference test has the highest fixing probabilities for a given failure rate and on average resolves a larger number of integer parameters than the per element ratio or projector test. Although this does per se not mean that the per element difference test is better than the other two tests, it is a very strong indicator that it is the best choice among the three options for GNSS positioning applications, where the goal is to obtain a good positioning quality. In the remainder of this dissertation, the per element ratio test and projector test are therefore no longer discussed. The capabilities of the per element difference test, the conventional difference test, and integer bootstrapping based subset selection schemes for reliable GNSS positioning are discussed in detail in Chapter 6.

\subsection{Fixed Failure Rate Critical Values}

For many realizations of integer aperture estimators and generalized integer aperture estimators, including all the examples presented in Section 3.2, Section 5.3, and Section 5.4, a single scalar critical value $\mu$ is used to determine the size and shape of the aperture region $\Omega$ or the selective aperture regions $\Omega_{\mathcal{J}}, \forall \mathcal{J} \in \mathfrak{I}$, defining the set $\mathcal{I}$ of integer parameters that are resolved. In Tiberius and de Jonge (1995), Wei and Schwarz (1995), Han and Rizos (1996), and Parkins (2011), different numerical values for the critical value $\mu$ are proposed for the difference test and the ratio test that seem to lead to good results for specific GNSS applications. In a practical system, however, not the critical value $\mu$ itself is the design parameter, but $\mu$ should rather be chosen such that the properties of the integer estimates $\check{\boldsymbol{a}}$ are as good as possible for the user application. In GNSS positioning applications, where incorrect ambiguity estimates generally lead to large errors in the user position, it is therefore a reasonable choice to use the critical value $\mu$ to control the failure rate $P_{\mathrm{F}}$. It is shown in Verhagen and Teunissen (2013) that for the ratio test integer aperture estimator a fixed critical value $\mu$ in most cases either results in too frequent incorrect ambiguity estimates or is overly conservative, which leads to an unnecessarily small acceptance rate of the integer solution. The fixed failure rate approach proposed in Teunissen and Verhagen (2009) and Verhagen and Teunissen (2013) is thus to adapt the critical value $\mu$ to the current situation as described by the observation model such that a user defined maximum tolerable failure rate is obtained. In this section, a user friendly method to determine the model dependent fixed failure rate critical value $\mu$ by means of a functional approximation is presented, see also Brack (2015).

\subsubsection{Relation Between Critical Value and Failure Rate}

Let $f(\cdot): \mathbb{R}^{1+n q+p q+q q} \mapsto \mathbb{R}$ be the function that describes the relation between the critical value $\mu$, the system model as given by $\boldsymbol{A}, \boldsymbol{B}$, and $\boldsymbol{Q}$, cf. Chapter 2 , and the failure rate $P_{\mathrm{F}} \in\left[0, P_{\mathrm{F}, \mathrm{ILS}}\right]$ 
for a specific estimator as

$$
\mu=f\left(P_{\mathrm{F}}, \boldsymbol{A}, \boldsymbol{B}, \boldsymbol{Q}\right) .
$$

Computing the fixed failure rate critical value $\mu$ means to evaluate this function for the user defined $P_{\mathrm{F}}$ and the given system model. The description of the system model could equivalently be replaced by $\boldsymbol{Q}_{\hat{a}}$ in this problem. Thinking about how the critical value $\mu$ affects the failure rate of the examples of (generalized) integer aperture estimators that were presented earlier clearly shows that $f(\cdot)$ can generally not be easily formulated. The function $f(\cdot)$ is investigated in more detail for the difference test integer aperture estimator (3.32) and the per element difference test generalized integer aperture estimator $(5.33)$. Let $f_{\boldsymbol{A}, \boldsymbol{B}, \boldsymbol{Q}}(\cdot): \mathbb{R} \mapsto \mathbb{R}$ be identical to the function $f(\cdot)$ evaluated at a specific system model, so that $\mu=f_{\boldsymbol{A}, \boldsymbol{B}, \boldsymbol{Q}}\left(P_{\mathrm{F}}\right)$. We start with analyzing some properties of $f_{\boldsymbol{A}, \boldsymbol{B}, \boldsymbol{Q}}(\cdot)$ for the difference test:

Given a critical value $\mu \geq 0$ and the corresponding failure and success rates $P_{\mathrm{F}}$ and $P_{\mathrm{S}}$, we consider $\mu^{\prime}>\mu$. Further, let $\mathcal{I}$ be the index set corresponding to $\mu$ and $\mathcal{I}^{\prime}$ the one corresponding to $\mu^{\prime}$. We can distinguish the following cases:

- If $\check{\boldsymbol{a}}_{\mathrm{ILS}}=\boldsymbol{a}$ and $\mathcal{I}=\{1, \ldots, n\}$ (success), it can now happen that $\mathcal{I}^{\prime}=\{1, \ldots n\}$ (success) or $\mathcal{I}^{\prime}=\emptyset$ (undecided).

- If $\check{\boldsymbol{a}}_{\mathrm{ILS}} \neq \boldsymbol{a}$ and $\mathcal{I}=\{1, \ldots, n\}$ (failure), it can now happen that $\mathcal{I}^{\prime}=\{1, \ldots n\}$ (failure) or $\mathcal{I}^{\prime}=\emptyset$ (undecided).

- If $\mathcal{I}=\emptyset$ (undecided), then $\mathcal{I}^{\prime}=\emptyset$ (undecided).

Integrating the pdf of $\hat{\boldsymbol{a}}$ over all possible realizations leads to the following conclusions. The failure rate of the difference test is decreasing with increasing critical value. The success rate of the difference test is decreasing with increasing critical value.

Similarly, we can analyze the per element difference test:

Given a critical value $\mu \geq 0$ and the corresponding failure and success rates $P_{\mathrm{F}}$ and $P_{\mathrm{S}}$, we consider $\mu^{\prime}>\mu$. Further, let $\mathcal{I}$ be the index set corresponding to $\mu$ and $\mathcal{I}^{\prime}$ the one corresponding to $\mu^{\prime}$. For the two random index sets it always holds that $\mathcal{I}^{\prime} \subseteq \mathcal{I}$. We can distinguish the following cases:

- If $\check{\boldsymbol{a}}_{\mathrm{ILS}, \mathcal{I}}=\boldsymbol{a}_{\mathcal{I}}$ and $\mathcal{I} \neq \emptyset$ (success), it can now happen that $\check{\boldsymbol{a}}_{\mathrm{ILS}, \mathcal{I}^{\prime}}=\boldsymbol{a}_{\mathcal{I}^{\prime}}$ and $\mathcal{I}^{\prime} \neq \emptyset$ (success) or $\mathcal{I}^{\prime}=\emptyset$ (undecided).

- If $\check{\boldsymbol{a}}_{\mathrm{ILS}, \mathcal{I}} \neq \boldsymbol{a}_{\mathcal{I}}$ and $\mathcal{I} \neq \emptyset$ (failure), it can now happen that $\check{\boldsymbol{a}}_{\mathrm{ILS}, \mathcal{I}^{\prime}} \neq \boldsymbol{a}_{\mathcal{I}^{\prime}}$ and $\mathcal{I}^{\prime} \neq \emptyset$ (failure) or $\check{\boldsymbol{a}}_{\mathrm{ILS}, \mathcal{I}^{\prime}}=\boldsymbol{a}_{\mathcal{I}^{\prime}}$ and $\mathcal{I}^{\prime} \neq \emptyset$ (success) or $\mathcal{I}^{\prime}=\emptyset$ (undecided).

- If $\mathcal{I}=\emptyset$ (undecided), then $\mathcal{I}^{\prime}=\emptyset$ (undecided).

Integrating the pdf of $\hat{\boldsymbol{a}}$ over all possible realizations leads to the following conclusions. The failure rate of the per element difference test is decreasing with increasing critical value. The success rate is generally not a monotonic function of the critical value.

As a consequence, a smaller failure rate of the per element difference test does not necessarily imply a smaller success rate as it does for the conventional difference test. It is again noted that success is a weaker statement if the estimation of subsets of the integer parameters is possible. On the other hand, the failure rates of both estimators are decreasing with increasing critical value, meaning that $f_{\boldsymbol{A}, \boldsymbol{B}, \boldsymbol{Q}}(\cdot)$ is a strictly monotonically decreasing function and therefore bijective. This leads to a unique critical value $\mu=f_{\boldsymbol{A}, \boldsymbol{B}, \boldsymbol{Q}}\left(P_{\mathrm{F}}\right)$ for a user selected value of the failure rate $P_{\mathrm{F}} \in\left[0, P_{\mathrm{F}, \mathrm{ILS}}\right]$ and thus enables an unambiguous fixed failure rate implementation. Due to the complicated structure of the region that leads to a failure, also $f_{\boldsymbol{A}, \boldsymbol{B}, \boldsymbol{Q}}(\cdot)$ cannot be expressed in 
closed form. The relation can rather be found by evaluating failure rates for given critical values $\mu$ through Monte Carlo integration, i.e., by evaluating the inverse function $P_{\mathrm{F}}=f_{\boldsymbol{A}, \boldsymbol{B}, \boldsymbol{Q}}^{-1}(\mu)$. The fixed failure rate critical value can be determined by iteratively refining an initial guess, which means by applying a root finding method such as bisection to $f_{\boldsymbol{A}, \boldsymbol{B}, \boldsymbol{Q}}^{-1}(\mu)-\gamma$, where $\gamma$ is the value of the aimed at failure rate.

With this strategy, the critical value can be computed for any system model and failure rate. Due to the high computational burden involved, this approach might not be feasible for many applications, such as real-time GNSS positioning. Since we aim at a constant failure rate $P_{\mathrm{F}}$, it would be very helpful if we could describe the influence of the system model on the critical value $\mu$ for this one specific value of $P_{\mathrm{F}}$ in a simpler way. To this end, let $f_{P_{\mathrm{F}}}(\cdot): \mathbb{R}^{n q+p q+q q} \mapsto \mathbb{R}$ be identical to $f(\cdot)$ evaluated at a specific failure rate, so that $\mu=f_{P_{\mathrm{F}}}(\boldsymbol{A}, \boldsymbol{B}, \boldsymbol{Q})$. An easy-to-evaluate approximation of $f_{P_{\mathrm{F}}}(\cdot)$ is presented in Section 5.5.2.

\subsubsection{Functional Approximation of Critical Values}

The basic idea to simplify the evaluation of $f_{P_{\mathrm{F}}}(\cdot)$ is to find a function $f_{\gamma}^{\text {app }}(\cdot)$ that can be used to compute approximate fixed failure rate critical values, but is much easier to evaluate. It is noted that the subscript $P_{\mathrm{F}}$ is replaced by $\gamma$, since the function is formulated for a specific value of the aimed at failure rate $P_{\mathrm{F}}=\gamma$, which is generally not exactly obtained. A possible strategy to define $f_{\gamma}^{\text {app }}(\cdot)$ could be to find an analytical approximation of the failure rate $P_{\mathrm{F}}$ for a given critical value $\mu$ and system model, so that the time consuming Monte Carlo integration can be avoided. In this dissertation, a different approach is followed, which is to simplify $f_{P_{\mathrm{F}}}(\cdot)$ by drastically reducing the dimension of its argument. This reduction generally goes along with a loss of information, so that the true fixed failure rate critical values $\mu$ can no longer be computed. It is aimed at finding the smallest possible vector of parameters $\boldsymbol{p}$, such as the dimension $n$ of $\boldsymbol{a}$, the integer least-squares failure rate $P_{\mathrm{F}, \mathrm{ILS}}$, or the ambiguity dilution of precision (ADOP, see Teunissen (1997c)), that captures the system characteristics that are relevant for describing the relation $f_{P_{\mathrm{F}}}(\cdot)$ for a given $P_{\mathrm{F}}=\gamma$ to a sufficient amount of accuracy as

$$
f_{\gamma}^{\mathrm{app}}(\boldsymbol{p}) \approx f_{P_{\mathrm{F}}}(\boldsymbol{A}, \boldsymbol{B}, \boldsymbol{Q})=\mu
$$

If the dimension of $\boldsymbol{p}$ is small enough, the function $f_{\gamma}^{\text {app }}(\cdot)$ can then either be sampled and stored in a look-up table as proposed in Verhagen and Teunissen (2013), or it can be described using a closed form expression (Brack 2015; Wang and Verhagen 2015; Hou et al. 2016). We follow the latter approach.

Let the function $f_{\gamma}^{\text {app }}(\cdot)$ be described through the vector of coefficients $\boldsymbol{\xi}^{*}$, which could for example be the coefficients of a polynomial. Assuming that the general structure of $f_{\gamma}^{\text {app }}(\cdot)$ is a priori known, we are thus only required to determine $\boldsymbol{\xi}^{*}$ in order to define $f_{\gamma}^{\text {app }}(\cdot)$. Further, let $g_{\gamma}^{\text {app }}(\cdot)$ be a function that is identical to $f_{\gamma}^{\text {app }}(\cdot)$ but for which the dependency on the vector of coefficients $\boldsymbol{\xi}$ is made explicit, so that $g_{\gamma}^{\text {app }}\left(\boldsymbol{\xi}^{*}, \boldsymbol{p}\right)=f_{\gamma}^{\text {app }}(\boldsymbol{p})$.

From simulations with many different system models that capture all situations that are relevant to the user, we obtain a data set with $K$ pairs of system parameters $\boldsymbol{p}^{k}$ and the corresponding critical values $\mu^{k}, k=1, \ldots, K$, for the aimed at failure rate $P_{\mathrm{F}}=\gamma$. The goal is now to find the vector of coefficients $\boldsymbol{\xi}^{*}$ such that the function $f_{\gamma}^{\text {app }}(\cdot)$ describes the relation between these data pairs as accurately as possible. As the dimension of $\boldsymbol{\xi}$ is much smaller than $K$, this results in a curve fitting problem. Moreover, as the user defined failure rate must not be exceeded, the approximate critical values as provided by $f_{\gamma}^{\text {app }}(\boldsymbol{p})$ have to be larger or equal to the true fixed failure rate critical 
values. This means that $\boldsymbol{\xi}^{*}$ has to be chosen such that $f_{\gamma}^{\text {app }}\left(\boldsymbol{p}^{k}\right) \geq \mu^{k}, k=1, \ldots, K$. Using the least-squares criterion, this fitting problem is an inequality constrained optimization problem (see Section A4) that reads

$$
\boldsymbol{\xi}^{*}=\underset{\boldsymbol{\xi} \in \mathbb{R}^{\operatorname{dim}(\boldsymbol{\xi})}}{\operatorname{argmin}} \sum_{k=1}^{K}\left(g_{\gamma}^{\mathrm{app}}\left(\boldsymbol{\xi}, \boldsymbol{p}^{k}\right)-\mu^{k}\right)^{2} \quad \text { s.t. } \quad \mu^{k}-g_{\gamma}^{\mathrm{app}}\left(\boldsymbol{\xi}, \boldsymbol{p}^{k}\right) \leq 0, k=1, \ldots, K,
$$

where $g_{\gamma}^{\text {app }}(\cdot)$ is assumed to be defined for every $\boldsymbol{\xi} \in \mathbb{R}^{\operatorname{dim}(\boldsymbol{\xi})}$. The Karush-Kuhn-Tucker (KKT) primal and dual feasibility (PF and DF) and complementary slackness (CS) conditions are given by

PF: $\quad \mu^{k}-g_{\gamma}^{\text {app }}\left(\boldsymbol{\xi}^{*}, \boldsymbol{p}^{k}\right) \leq 0, \quad k=1, \ldots, K$

DF: $\quad \nabla_{\boldsymbol{\xi}}\left(\sum_{k=1}^{K}\left(g_{\gamma}^{\mathrm{app}}\left(\boldsymbol{\xi}^{*}, \boldsymbol{p}^{k}\right)-\mu^{k}\right)^{2}\right)$

$$
+\sum_{k=1}^{K} \nabla_{\boldsymbol{\xi}}\left(\mu^{k}-g_{\gamma}^{\mathrm{app}}\left(\boldsymbol{\xi}^{*}, \boldsymbol{p}^{k}\right)\right) u^{k}=\mathbf{0}, \quad \text { with } u^{k} \geq 0, k=1, \ldots, K
$$

CS: $u^{k}\left(\mu^{k}-g_{\gamma}^{\text {app }}\left(\boldsymbol{\xi}^{*}, \boldsymbol{p}^{k}\right)\right)=0, \quad k=1, \ldots, K$,

where $u^{k} \geq 0, k=1, \ldots, K$, are the non-negative Lagrangian multipliers and $\nabla_{\boldsymbol{\xi}}$ is the gradient with respect to $\xi$. For any optimization problem with objective and constraint functions that are differentiable at $\boldsymbol{\xi}^{*}$, the KKT conditions are necessary conditions for $\boldsymbol{\xi}^{*}$ to be a local optimizer of (5.45), given that certain constraint qualifications are fulfilled, so that strong duality holds. In our problem, usually only one of the Lagrangian multipliers $u^{k}$ is non-zero, which is the one that corresponds to the active inequality constraint, i.e., the data point $\mu^{k}$ that lies on $f_{\gamma}^{\text {app }}(\cdot)$ so that $\mu^{k}=g_{\gamma}^{\text {app }}\left(\boldsymbol{\xi}^{*}, \boldsymbol{p}^{k}\right)$. One version of the constraint qualifications is that the gradients of the active inequality constraints are linear independent, which is always true for only one active constraint.

Under the condition that the structure of $g_{\gamma}^{\text {app }}(\cdot)$ is chosen such that both the cost function $\sum_{k=1}^{K}\left(g_{\gamma}^{\text {app }}\left(\boldsymbol{\xi}, \boldsymbol{p}^{k}\right)-\mu^{k}\right)^{2}$ and the active inequality constraints $\mu^{k}-g_{\gamma}^{\text {app }}\left(\boldsymbol{\xi}, \boldsymbol{p}^{k}\right)$ are convex, (5.45) is a convex optimization problem. This implies that Slater's constraint qualification can be employed, which state that the interior of the feasible region of $\boldsymbol{\xi}$ as defined by the inequality constraints has to be non-empty. In our problem, this is the case if $\boldsymbol{\xi}$ can be chosen such that $g_{\gamma}^{\text {app }}\left(\boldsymbol{\xi}, \boldsymbol{p}^{k}\right)$ is strictly larger than $\mu^{k}, k=1, \ldots, K$, i.e., that it is a strict upper bound for all data points. Given that Slater's condition is fulfilled and that the objective and constraint functions are differentiable at a KKT point $\boldsymbol{\xi}^{*}$, then $\boldsymbol{\xi}^{*}$ is the global optimizer of (5.45). The optimal dual variables $u^{k}$, $k=1, \ldots, K$, from which the primal solution $\boldsymbol{\xi}^{*}$ is reconstructed, can be found with standard subgradient based dual methods, where the non-negativity of all $u^{k}$ has to be taken care of. Problem (5.45) is for instance convex, if $g_{\gamma}^{\text {app }}(\cdot)$ is a polynomial with $\boldsymbol{\xi}$ as coefficients.

As a first step, we have to create the data pairs $\left\{\boldsymbol{p}^{k}, \mu^{k}\right\}$ for many different system models that cover the whole span of characteristics that the user is interested in. This can either mean to consider a very wide spectrum of different system models so as to obtain a preferably generally valid description, maybe at the cost of higher approximation errors, but it can also be limited to a very specific case, e.g., short baseline GNSS positioning with a specific choice of signals in a certain geographic area. The results presented in the following are based on the GNSS positioning examples as described in Table 5.1, where the per-element difference test is applied in the LAMBDA decorrelated space. In contrast to the conventional difference test, a different integer transformation generally results in different fixed failure rate critical values for the per element difference test, meaning that the so found functional approximation is only valid in a decorrelated space. One parameter to be chosen for $\boldsymbol{p}$ that is most likely suitable to describe the system characteristics is the 


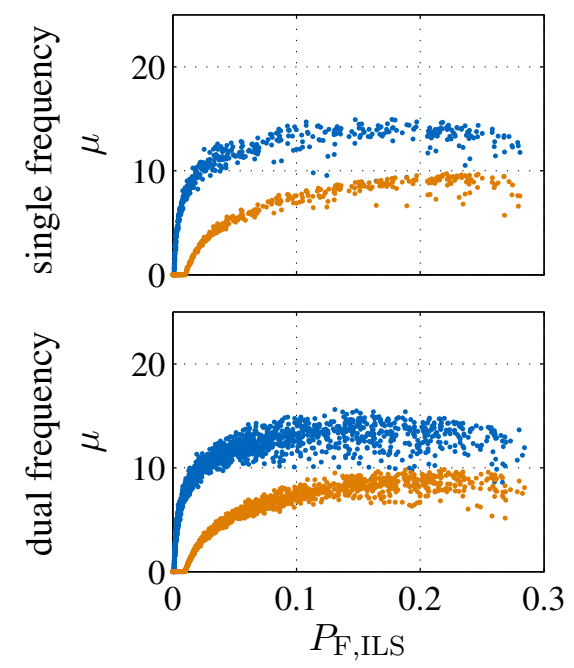

(a) GPS L1/L1+L2
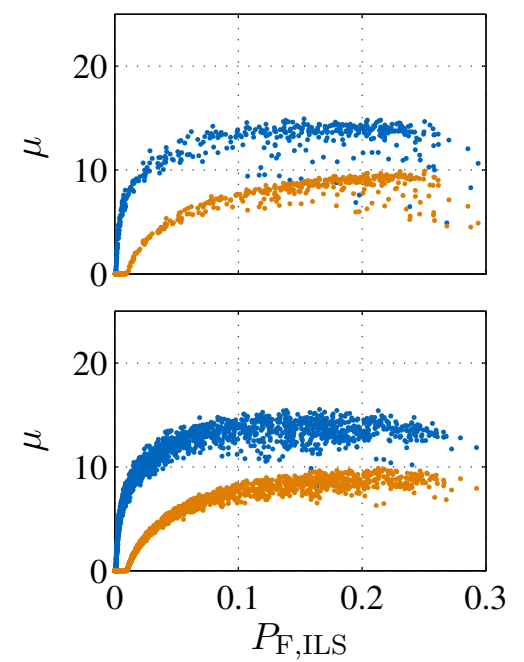

(b) Galileo E1/E1+E5a
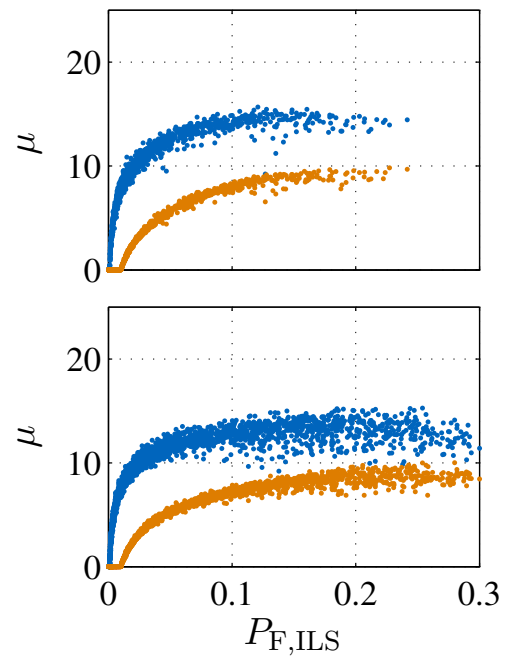

(c) Combined GPS L1/L1+L2 and Galileo E1/E1+E5a

Figure 5.6: Relation between integer least-squares failure rate $P_{\mathrm{F}, \mathrm{ILS}}$ and critical value $\mu$ for the per element difference test for various GNSS positioning models and a fixed failure rate of $P_{\mathrm{F}}=0.1 \%$ (blue) and $P_{\mathrm{F}}=1.0 \%$ (orange).

integer least-squares failure rate $P_{\mathrm{F}, \mathrm{ILS}}$, as this is an indicator for the strength of the system model regarding the ambiguity resolution capabilities. We define $p=P_{\mathrm{F}, \mathrm{ILS}}$, meaning that no further system parameters are required to describe the fixed failure rate critical value. Furthermore, since integer least-squares (+ selection) is the underlying (partial) integer estimator for the (per element) difference test, $P_{\mathrm{F}, \mathrm{ILS}}$ already defines one property of $f_{\gamma}^{\text {app }}(\cdot)$. If the user defined maximum tolerable failure rate is larger than $P_{\mathrm{F}, \mathrm{ILS}}$, we can always accept the full integer least-squares solution, i.e., the critical value is chosen as $\mu \leq 0$. This defines the zero crossing of $f_{\gamma}^{\text {app }}(\cdot)$ as

$$
f_{\gamma}^{\operatorname{app}}(\gamma)=0
$$

In Figure 5.6, the relation between $P_{\mathrm{F}, \mathrm{ILS}}$ and the resulting critical values $\mu$ is shown for the per element difference test separately for different available signals. Each data point is computed via Monte Carlo integration as described above and represents a different system model. Although the subfigures represent very different model characteristics, as for instance the number of integer parameters ranges from $n \in[5,13]$ for the single system, single frequency case to $n \in[18,42]$ for the combined system, dual frequency case, the basic relation is quite similar. This shows that $P_{\mathrm{F}, \mathrm{ILS}}$ is indeed a relevant parameter, but $n$ is not. It is noted that when determining the critical value $\mu$ for a given system model and failure rate via Monte Carlo integration, a search for $\check{\boldsymbol{a}}_{\mathrm{ILS}}$ and the respective counter hypotheses $\overline{\boldsymbol{a}}^{i}$ has to be performed for every randomly generated sample of $\hat{\boldsymbol{a}}$. The relation (5.42) for setting the size of the search space can obviously not be used, as is depends on $\mu$ itself. It is therefore necessary to find a somewhat more conservative value of the search radius. The integer bootstrapping based search radius in (5.41) would lead to a valid counter hypothesis for each element of $\check{\boldsymbol{a}}_{\mathrm{ILS}}$ and could therefore be used for an arbitrary value of the aimed at failure rate, but is usually much too conservative for a given, specific failure rate constraint, leading to unnecessarily long search times. The results in Figure 5.6 indicate that for $P_{\mathrm{F}}=0.1 \%$, the critical value $\mu$ does not or very rarely assume values $>16$. The size of the search space $\lambda^{2}$ 
can therefore be empirically limited to

$$
\lambda^{2}=\left\|\hat{\boldsymbol{a}}-\check{\boldsymbol{a}}_{\mathrm{ILS}}\right\|_{\boldsymbol{Q}_{\hat{\boldsymbol{a}}}}^{2}+16 \leq\left\|\hat{\boldsymbol{a}}-\check{\boldsymbol{a}}_{\mathrm{IB}}\right\|_{\boldsymbol{Q}_{\hat{\boldsymbol{a}}}}^{2}+16
$$

for this application.

Considering (5.47) and the results in Figure 5.6, the function $g_{\gamma}^{\text {app }}(\cdot)$ is chosen as

$$
g_{\gamma}^{\mathrm{app}}\left(\boldsymbol{\xi}, P_{\mathrm{F}, \mathrm{ILS}}\right)=\xi_{1} \log \left(\xi_{2}\left(P_{\mathrm{F}, \mathrm{ILS}}-\gamma\right)+1\right) .
$$

For system models with $P_{\mathrm{F}, \mathrm{ILS}}<\gamma$, any $\mu \leq 0$ is equally correct. Accordingly, these models have to be excluded when evaluating (5.45) so that the cost function is not disturbed and no invalid constraints are imposed. The parameter $\xi_{2}$ has to be constrained to $\xi_{2}>-\frac{1}{1-\gamma}$ so that the argument of the logarithm is non-negative for $P_{\mathrm{F}, \mathrm{ILS}} \in[\gamma, 1]$. In order for $g_{\gamma}^{\text {app }}\left(\boldsymbol{\xi}, P_{\mathrm{F}, \mathrm{ILS}}\right)$ to resemble the numerically computed critical values in Figure 5.6, $\xi_{1}$ has to be non-negative. Since the critical values $\mu$ are strictly positive for $P_{\mathrm{F}, \mathrm{ILS}}>\gamma$, the optimal $\xi_{2}^{*}$ is positive as well. The restrictions $\xi_{1} \geq$ 0 and $\xi_{2} \geq 0$ could be included as additional constraints in (5.45). For the relevant cases $\left.\gamma \in\right] 0,1[$ these constraints are, however, not active and the corresponding Lagrangian multipliers would be zero, so that the KKT conditions in (5.46) are still valid. With $\xi_{1} \geq 0$, the inequality constraints in (5.45) are convex in $\xi$, but the cost function is a sum of quasiconvex functions and thus not convex. We can therefore either hope to converge to the correct KKT point by properly initializing $\boldsymbol{\xi}$, or we can further utilize certain properties of the problem. The feasible set of problem (5.45) is not only a convex set for (5.49), but also a reverse normal set in $\mathbb{R}_{+}^{2}$, meaning that for $\boldsymbol{\xi}, \boldsymbol{\xi}^{\prime} \in \mathbb{R}^{2}$, if $\boldsymbol{\xi}$ is part of the feasible set and $\boldsymbol{\xi}^{\prime} \geq \boldsymbol{\xi}$, then also $\boldsymbol{\xi}^{\prime}$ is part of the feasible set. This is true since $g_{\gamma}^{\text {app }}\left(\boldsymbol{\xi}, P_{\mathrm{F}, \mathrm{ILS}}\right)$ is increasing in $\boldsymbol{\xi}$. For the same reason, the cost function in (5.45) is increasing in $\boldsymbol{\xi}$ for all $\boldsymbol{\xi}$ that are in the feasible set (the functions $g_{\gamma}^{\text {app }}\left(\boldsymbol{\xi}, P_{\mathrm{F}, \mathrm{ILS}}^{k}\right), k=1, \ldots, K$, are greater or equal to $\mu^{k}$ within the feasible set, so that increasing the functions increases each of the non-negative terms of the sum). Increasing functions and normal sets have been introduced to mathematical economics in Makarov and Rubinov (1977). If $g_{\gamma}^{\text {app }}\left(\boldsymbol{\xi}, P_{\mathrm{F}, \mathrm{ILS}}^{k}\right)$ would also be increasing outside of the feasible region, which it is not, the problem could be solved within the framework of monotonic optimization (Tuy 1999, 2000). However, we can still make use of some of the ideas. A direct consequence of the two aforementioned properties is that the global optimum $\boldsymbol{\xi}^{*}$ is obtained at the boundary of the feasible region, which contains its Pareto efficient points. It is very easy to evaluate, whether or not a certain value $\boldsymbol{\xi}$ is feasible or not. A possible approach to solve the optimization problem is thus to simply sample the boundary of the feasible region and to chose the optimizer $\xi$ out of these samples. Figure 5.7 shows the feasible region of $\boldsymbol{\xi}$ for $\gamma=0.1 \%$. The Pareto efficient points are shown in blue, and the global optimizer $\boldsymbol{\xi}^{*}$ in orange.

The union of all data pairs from Figure 5.6 is shown in Figure 5.8 together with the resulting functional approximation $g_{\gamma}^{\text {app }}\left(\boldsymbol{\xi}^{*}, P_{\mathrm{F}, \mathrm{ILS}}\right)=f_{\gamma}^{\text {app }}\left(P_{\mathrm{F}, \mathrm{ILS}}\right)$ for $\gamma=0.1 \%$ and $\gamma=1.0 \%$. The function parameters $\xi^{*}$ for the per element difference test are determined as

$$
\begin{array}{ll}
\boldsymbol{\xi}^{*}=[2.45,5,074]^{\mathrm{T}} & \text { for } \gamma=0.1 \% \\
\boldsymbol{\xi}^{*}=[2.82,214]^{\mathrm{T}} & \text { for } \gamma=1.0 \%
\end{array}
$$

The functions $f_{\gamma}^{\text {app }}\left(P_{\mathrm{F}, \mathrm{ILS}}\right)$ have the desired properties. They resemble the characteristics of the fixed failure rate critical values while being a conservative approximation. They are a tight upper bound, at least for relatively small values of $P_{\mathrm{F}, \mathrm{ILS}}$.

The framework presented in this section can also be applied to other (generalized) integer aperture estimators that are based on different acceptance tests. The resulting function may then 

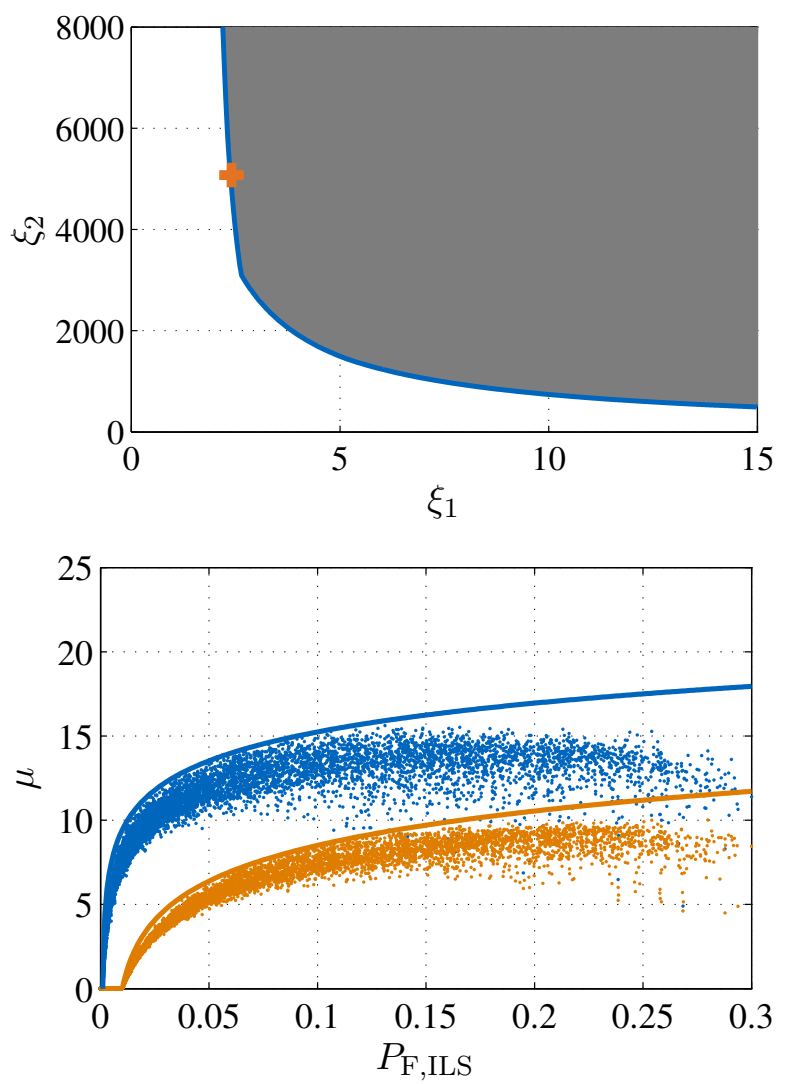

Figure 5.7: The feasible region of $\boldsymbol{\xi}$ for the per element difference test and $\gamma=0.1 \%$ is shown in gray, the Pareto efficient points in blue, and the optimizer $\xi^{*}$ in orange.

Figure 5.8: The dots show the relation between the integer least-squares failure rate $P_{\mathrm{F}, \mathrm{ILS}}$ and the critical value $\mu$ for the per element difference test for a fixed failure rate of $P_{\mathrm{F}}=0.1 \%$ (blue) and $P_{\mathrm{F}}=1.0 \%$ (orange). The lines show the approximating functions $f_{\gamma}^{\text {app }}\left(P_{\mathrm{F}, \mathrm{ILS}}\right)$.

of course be of a different form and depend on a different set of relevant system parameters $\boldsymbol{p}$. For instance, the conventional ratio test requires the total number of integer parameters as an input in addition to the integer least-squares failure rate in order to allow for a meaningful representation (Verhagen and Teunissen 2013).

\subsubsection{Approximated vs. True Fixed Failure Rate Critical Values}

The critical values $\mu$ that are computed with the functional approximation as

$$
\mu=f_{\gamma}^{\text {app }}\left(P_{\mathrm{F}, \mathrm{ILS}}\right)
$$

suffer from two error sources. The first one is due to the fact that $f_{\gamma}^{\text {app }}\left(P_{\mathrm{F}, \mathrm{ILS}}\right)$ is designed to provide an upper bound for the true fixed failure rate critical values. The second one comes from the integer least-squares failure rate $P_{\mathrm{F}, \mathrm{ILS}}$, which cannot be computed in closed form, so that $f_{\gamma}^{\text {app }}(\cdot)$ has to be evaluated at an approximate value of $P_{\mathrm{F}, \mathrm{ILS}}$ in practice. If an upper bound like the integer bootstrapping failure rate $P_{\mathrm{F}, \mathrm{IB}}$ is used to approximate $P_{\mathrm{F}, \mathrm{ILS}}$, both effects cause increased critical values, leading to failure rates that are smaller than required with the fixed failure rate approach. Figure 5.9 illustrates the translation of these errors to the resulting failure rates. the blue curve in the right subfigure shows $f_{0.1 \%}^{\text {app }}\left(P_{\mathrm{F}, \mathrm{ILS}}\right)$, and the orange and green curves in the left subfigure show the relation between the critical value $\mu$ and the resulting failure rate $P_{\mathrm{F}}$ for two specific system models. Because of the common vertical axis, the impact on the failure rate $P_{\mathrm{F}}$ caused by the use of $f_{0.1 \%}^{\text {app }}(\cdot)$ and $P_{\mathrm{F}, \mathrm{IB}}$ is directly visible. Although the integer bootstrapping failure rate is usually a tight upper bound of $P_{\mathrm{F}, \mathrm{ILS}}$, the mismatch may still affect the failure rate, especially for small values of $P_{\mathrm{F}, \mathrm{ILS}}$, where $f_{0.1 \%}^{\text {app }}\left(P_{\mathrm{F}, \mathrm{ILS}}\right)$ is very sensitive to changes in the argument. We can see that both approximations lead to error rates $P_{\mathrm{F}}$ that are somewhat smaller than $0.1 \%$. 

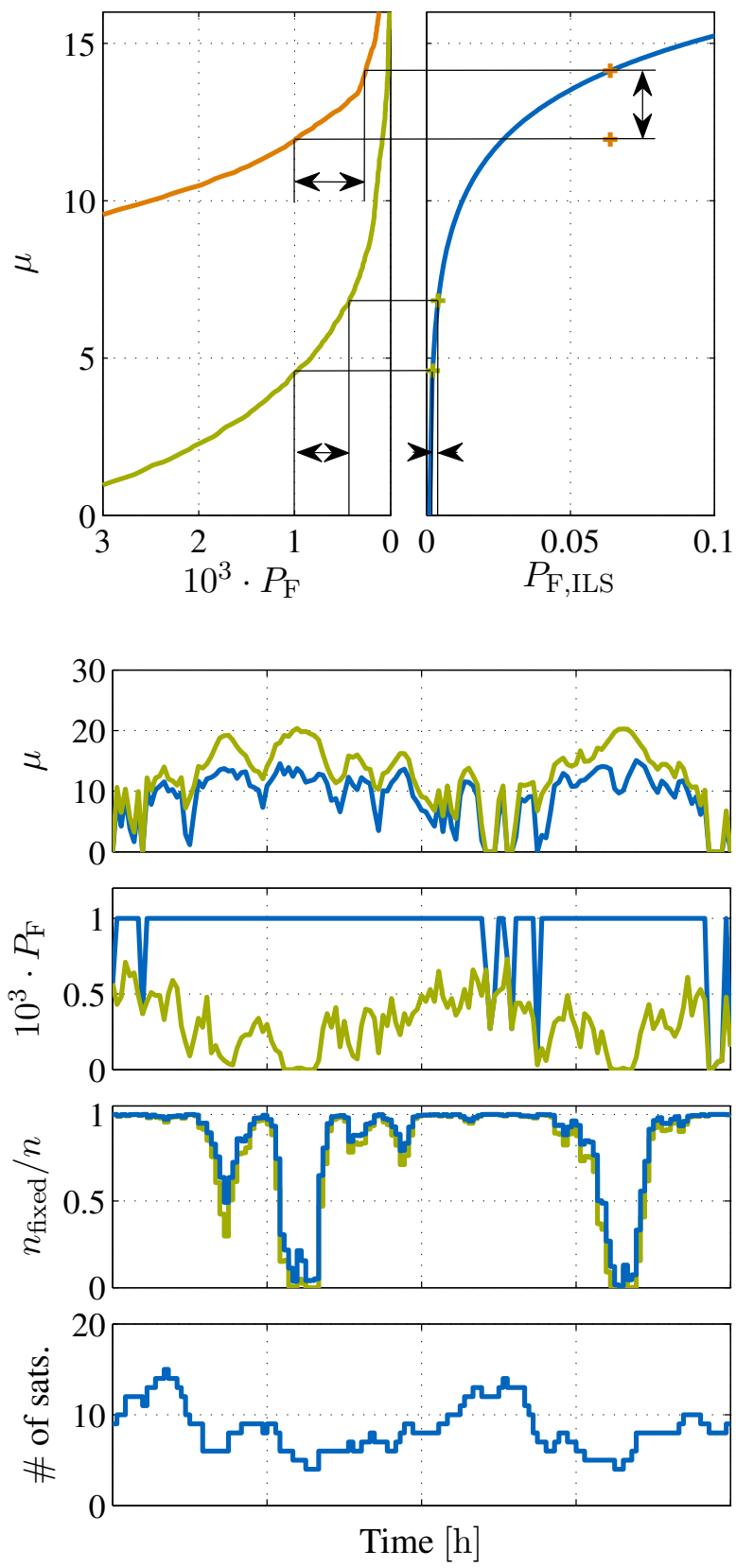

(a) GPS L1+L2
Figure 5.9: Examples of how an error in the input argument $P_{\mathrm{F}, \mathrm{ILS}}$ (green) and an error introduced by upper bounding (orange) translate into an error in the resulting $P_{\mathrm{F}}$; the blue curve shows $f_{0.1 \%}^{\text {app }}\left(P_{\mathrm{F}, \mathrm{ILS}}\right)$; the green and orange curves show the relation between $\mu$ and the resulting failure rate $P_{\mathrm{F}}$ for two different system models.
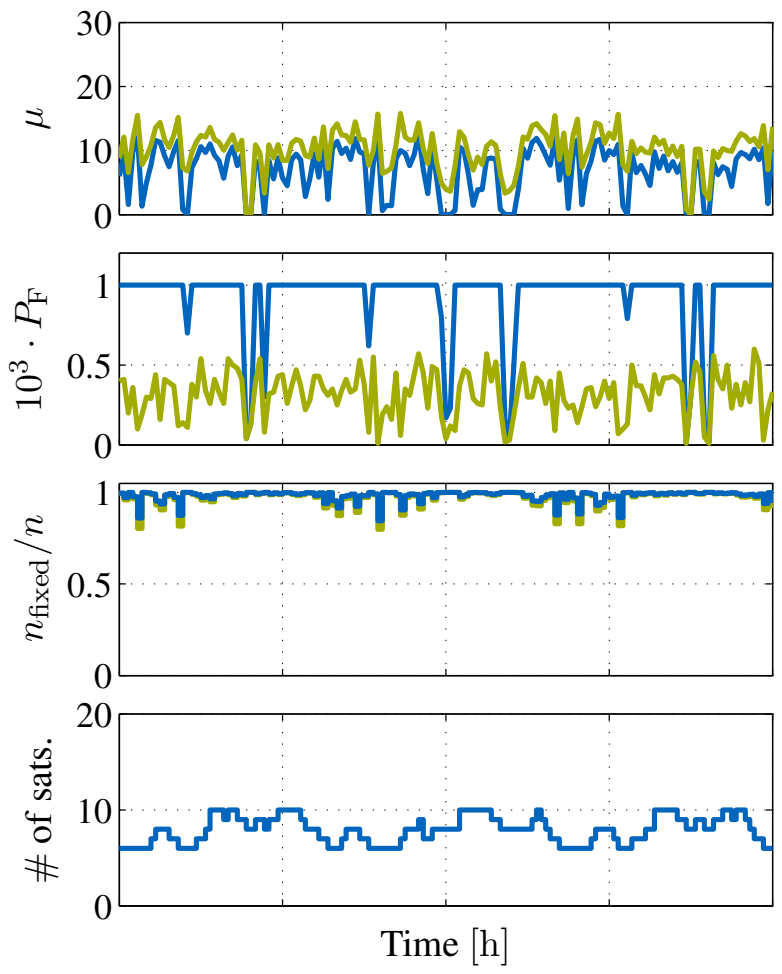

(b) Galileo E1+E5a

Figure 5.10: Comparison of approximated (green) and true (blue) fixed failure rate critical values $\mu$, resulting failure rates $P_{\mathrm{F}}$, and ratios of fixed ambiguities $n_{\text {fixed }} / n$ for an aimed at failure rate of $0.1 \%$ with $24 \mathrm{~h}$ of simulated dual frequency observations; the number of visible satellites is shown in the last row.

The impact of using the approximated critical values $\mu=f_{0.1 \%}^{\text {app }}\left(P_{\mathrm{F}, \mathrm{IB}}\right)$ instead of the true fixed failure rate critical values is numerically evaluated for single epoch GNSS positioning with $24 \mathrm{~h}$ of dual frequency GPS and Galileo observations in the area of Munich, Germany. The standard deviation of the between receiver ionospheric zenith delay is assumed as $0.71 \mathrm{~cm}$, and the receiver noise is assumed as in Table 5.1 with a scaling factor of one. These models have not been used for determining $\boldsymbol{\xi}^{*}$, so that this experiment can be seen as a validation of the functional approximation.

The results for GPS in Figure 5.10 show that the green critical values generated by $f_{0.1 \%}^{\text {app }}\left(P_{\mathrm{F}, \mathrm{IB}}\right)$ are always larger or equal to the true fixed failure rate critical values from Monte Carlo integration 
in blue. For the true critical values, the corresponding failure rates are always $P_{\mathrm{F}}=0.1 \%$ per definition, except for the cases in which $P_{\mathrm{F}, \mathrm{ILS}}<0.1 \%$. In those cases, the resulting failure rate is equal to the integer least-squares failure rate, as the full integer least-squares solution is then directly accepted. The failure rates resulting from the approximate critical values are always smaller or equal to the ones from the true critical values. For all time instances with moderate values of $\mu$, where the function $f_{0.1 \%}^{\text {app }}\left(P_{\mathrm{F}, \mathrm{ILS}}\right)$ is a relatively sharp upper bound, see Figure 5.8, the difference between the true and approximate failure rates is roughly $0.05 \%$. It is noted that function values of $f_{0.1 \%}^{\text {app }}\left(P_{\mathrm{F}, \mathrm{IB}}\right) \approx 20$ correspond to $P_{\mathrm{F}, \mathrm{IB}} \approx 70 \%$, i.e., a loose lower bound for the aimed at failure rate is not critical, since these models are very weak with regard to ambiguity resolution anyway. It might be possible to adjust the parameter vector $\boldsymbol{\xi}$ defining the functional description such that the resulting failure rates are slightly closer to $0.1 \%$ by using a more restrictive data set for the function fitting. Experiments indicate, however, that these gains are not significant.

An indicator that can be used to assess the effect of the approximation on the performance of the per element difference test is the average ratio of resolved integer parameters $n_{\text {fixed }} / n$. Although the failure rates resulting from the approximate critical values are partly considerably smaller than required, the ratios of fixed ambiguities $n_{\text {fixed }} / n$ is hardly affected by the approximation, especially for strong system models, where $n_{\text {fixed }} / n$ is close to one. The low ratio values at $7-8 \mathrm{~h}$ and $20-21 \mathrm{~h}$ confirm that the impact of a loose approximation of large critical values is not too severe.

The corresponding results for Galileo verify what has been observed for GPS. The minimum number of satellites is slightly increased compared to GPS, resulting in generally stronger models. The true fixed failure rate critical values are limited to $\mu \leq 15$, which roughly corresponds to $P_{\mathrm{F}, \mathrm{ILS}} \leq 10 \%$. The maximum tolerable failure rate of $P_{\mathrm{F}}=0.1 \%$ is never exceeded with the approximate critical values, and the resulting ratios of fixed ambiguities are always very close to the ones from the true critical values. Moreover, they are close to one during the whole time span of $24 \mathrm{~h}$, which indicates a very good performance of the per element difference test for models of that type and strength. The capabilities of the per element difference test for GNSS positioning applications are further investigated in Chapter 6. 


\section{Reliable GNSS Positioning_Experimental Analysis}

The estimation of (a subset of) integer parameters in linear models, which are not necessarily GNSS related, is discussed in detail in Chapters 3, 4, and 5. Depending on the application at hand, measures like the success rate, the fixing rate, or the ratio of resolved integer parameters that are presented there may describe or at least be an indicator for the performance of a specific estimator. In this chapter we investigate the application of some of the estimators from Chapters 3, 4, and 5 to the problem of carrier-phase integer ambiguity resolution for GNSS positioning with system models as derived in Chapter 2. Measures that can be used to describe the quality of the position estimates are discussed in Section 6.1. These are assessed via numerical simulations for various single and combined system GPS and Galileo cases in Section 6.2. The results are verified with real GPS and BDS data collected at multiple continuously operating reference stations (CORSs) in the area of Perth, Australia. The main goal is to determine, if, when, and to what extent partial ambiguity resolution techniques can enhance the positioning capabilities compared to conventional full ambiguity resolution.
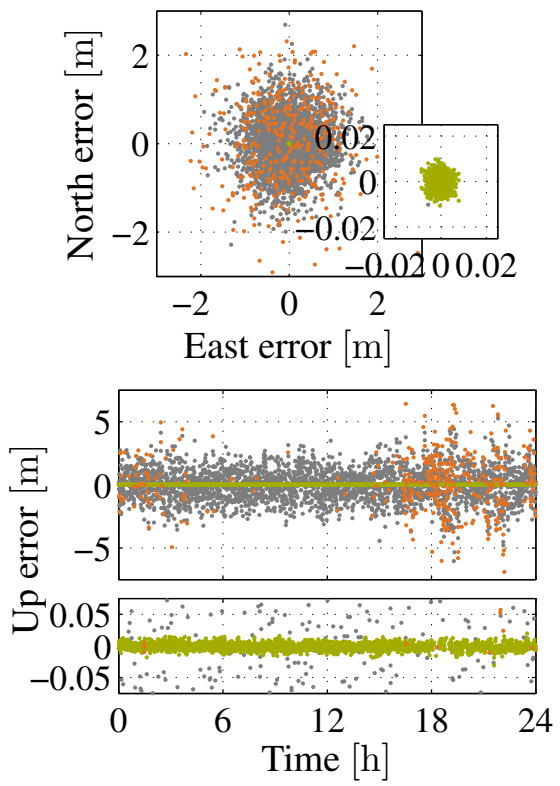

(a) GPS L1
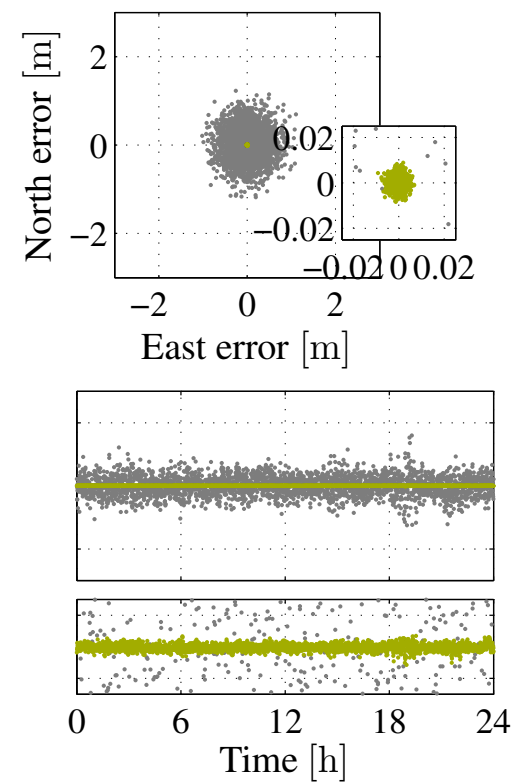

(b) GPS L1+L2
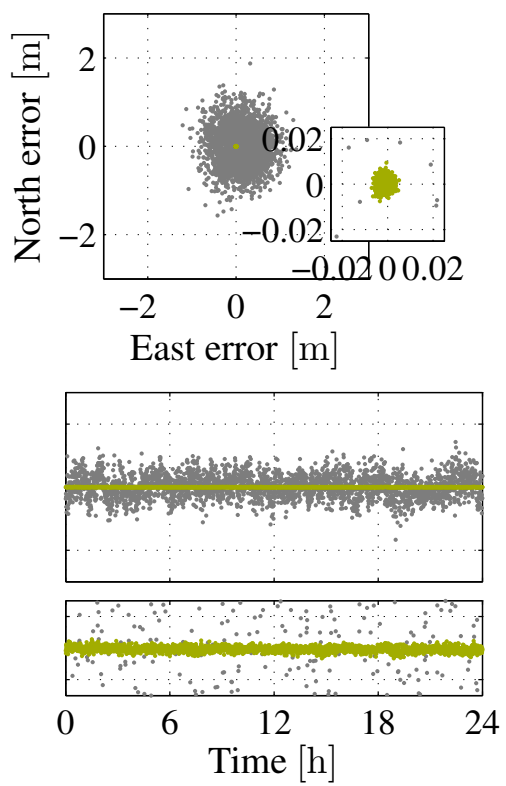

(c) GPS L1 and BDS B1

Figure 6.1: Instantaneous RTK positioning results for CUT0-CUTA during May 1, 2015; the positioning error is shown in gray for the float solution, and in green and orange for the ambiguity fixed solution with correct and incorrect integer estimates, respectively.

Figure 6.1 shows the positioning errors for three different examples. The data was collected at the stations CUT0, which is part of the multi-GNSS experiment (MGEX) of the IGS, and CUTA in Perth, Australia, during May 1, 2015. The two stations form a baseline of 8.4 meters, so that differential atmospheric delays can be assumed absent. The processing is done epoch-by-epoch, i.e., the temporal stability of the parameters is not utilized. The ambiguities are resolved using integer least-squares without taking care of any kind of reliability constraint. The results for sin- 
gle frequency GPS L1 confirm what is claimed earlier in this dissertation. The ambiguity fixed positioning precision with correct integer estimates is at centimeter or even sub-centimeter level, thereby two orders of magnitude better than the precision of the float position estimates. Incorrect ambiguity estimates generally lead to large positioning errors, often exceeding the error of the float solution. This demonstrates the importance of limiting the ambiguity failure rate.

Using dual frequency GPS L1+L2 leads to a higher precision of the float position estimates, and also a slightly better precision of the ambiguity fixed position estimates. More importantly, the ambiguity resolution capabilities of such a dual frequency short baseline case are drastically improved, so that no incorrect ambiguity estimates occur during the whole $24 \mathrm{~h}$. It seems that there is not much space for improvement with partial ambiguity resolution, but we have to keep in mind that these results are obtained in the absence of differential atmospheric delays, which is limiting the length of the baseline to at most a few kilometers. With longer baselines, when atmospheric delays have to be considered, thus weakening the underlying system model, partial ambiguity resolution may very well be a useful tool. This is investigated in Sections 6.2 and 6.3.

An empirical ambiguity success rate of $100 \%$ is also obtained for single frequency L1/B1 positioning when combining GPS and BDS due to the larger number of visible satellites. This is consistent with the study in Odolinski and Teunissen (2016), where the RTK positioning capabilities of dual frequency single GNSS and single frequency dual GNSS are compared. The benefit of combining multiple GNSS on the ambiguity resolution performance was predicted in Tiberius et al. (2002) for GPS and Galileo and demonstrated for GPS and BDS in Deng et al. (2014), He et al. (2014), Odolinski et al. (2014b), and Teunissen et al. (2014), for GPS and IRNSS in Zaminpardaz et al. (2017), for GPS, Galileo, BDS, and QZSS in Odolinski et al. (2015a), and for GPS, Galileo, QZSS, and IRNSS in Odijk et al. (2017). Instantaneous and reliable full ambiguity resolution on long baselines, however, is not possible (Tiberius et al. 2002) and fast solutions still remain difficult, even in such a combined GNSS case (Odolinski et al. 2014a, 2015b). Considering that for instance the per element acceptance tests seem to work very well for the combined GNSS case in terms of the fixing probability and the ratio of resolved integer parameters, see Section 5.4, partial ambiguity resolution is likely to be very helpful in order to further improve the performance of combined GNSS positioning.

\subsection{Positioning Precision, Ambiguity Reliability, and Time}

The positioning solution when using one of the estimators from Chapters 3, 4, or 5 for integer ambiguity resolution is fully described by the pdf of the estimated user position, see Sections 3.1.1, 3.2.1, 4.2, and 5.2. In order to analyze the performance of different ambiguity resolution schemes, it would, however, be beneficial if the quality of the positioning solution could be described by only a few relevant measures. Preferably, these measures are such that they can be easily determined, so that they can be provided to the user to inform him about how good he can expect the estimated position to be. If the integer solution is assumed non-stochastic as $\check{\boldsymbol{a}}=\boldsymbol{a}_{\mathcal{J}}$ with the deterministic index set $\mathcal{I}=\mathcal{J}$, then the (partially) ambiguity fixed estimate $\breve{b}$ is unbiased and its precision is fully described by the conditional covariance matrix $Q_{\hat{b} \mid \hat{a}_{\mathcal{J}}}$. If the index set $\mathcal{I}$ is random and the integer solution is assumed as $\check{\boldsymbol{a}}=\boldsymbol{a}_{\mathcal{I}}$, then $\boldsymbol{Q}_{\hat{\boldsymbol{b}} \mid \hat{\boldsymbol{a}}_{\mathcal{J}}}$ is generally not the covariance matrix of $\check{\boldsymbol{b}}$ given that $\mathcal{I}=\mathcal{J}$, but can still be used as an approximation of the precision once the index set $\mathcal{I}=\mathcal{J}$ has been determined by the estimator. As we have seen, incorrect ambiguity estimates $\check{\boldsymbol{a}}$ often lead to large position errors, so that $\boldsymbol{Q}_{\hat{b} \mid \hat{\boldsymbol{a}}_{\mathcal{J}}}$ can only be meaningful given that the failure rate $P_{\mathrm{F}}$ is sufficiently small. The performance measures that are analyzed within this chapter are therefore 
the positioning precision by means of $\boldsymbol{Q}_{\hat{b} \mid \hat{a}_{\mathcal{J}}}$, which is computed for a specific value $\mathcal{I}=\mathcal{J}$ of the index set, the ambiguity reliability by means of the failure rate $P_{\mathrm{F}}$, and the number of measurement epochs that are used. In order to visualize the computed formal precision, we define the scalar formal precision measure

$$
\alpha=\max \left\{\frac{\sigma_{\mathrm{E}}}{1 \mathrm{~cm}}, \frac{\sigma_{\mathrm{N}}}{1 \mathrm{~cm}}, \frac{\sigma_{\mathrm{U}}}{3 \mathrm{~cm}}\right\}
$$

where $\sigma_{\mathrm{E}}, \sigma_{\mathrm{N}}$, and $\sigma_{\mathrm{U}}$ are the standard deviations of the coordinate estimates in the local east, north, and up frame that follow from $\boldsymbol{Q}_{\hat{b} \mid \hat{\boldsymbol{a}}_{\mathcal{J}}}$. The smaller factor for $\sigma_{\mathrm{U}}$ accounts for the larger uncertainty that is usually observed for the up component.

The goal is of course to obtain fast, precise, and reliable solutions, which is often not easy to achieve. For instance, long observation time spans generally increase the reliability of ambiguity resolution and usually improve the precision of the float and fully fixed position, but do obviously not lead to fast results. In order to better understand the effect of model-driven and data-driven schemes for full and partial ambiguity resolution on GNSS positioning, feasible combinations of these three quality measures are analyzed for a long baseline example, in which the differential atmospheric delays are estimated. The results are presented in Figure 6.2. Rows correspond to different estimation schemes. For each scheme, three subfigures are shown, where in each subfigure two of the three measures are analyzed for a given value of the third measure. The left subfigure corresponds to a reliability of $P_{\mathrm{F}} \leq 0.1 \%$, the second subfigure to a precision of $\alpha \leq 10$, and the right subfigure to an observation span of at most 20 epochs. The orange and green dashed lines represent the float and fully fixed solution.

The left column therefore shows the results for reliable positioning, in which the failure rate is constrained so as to not exceed $0.1 \%$. The orange line shows the precision of the float solution over time, and the green line the precision of the fully ambiguity fixed solution. Both of them improve over time, and the difference of roughly two orders of magnitude after initialization becomes smaller over time. With model-driven full ambiguity resolution it takes 54 epochs until the model is strong enough to switch from the float to the fixed solution. With model-driven partial ambiguity resolution, the first ambiguity can already be resolved after 8 epochs. The partially fixed solution gradually improves over time with the increasing number of resolved ambiguities until the fully fixed precision is again reached after 54 epochs. For data-driven full ambiguity resolution, the fixed precision is obtained with the probability $P(\mathcal{I}=\{1, \ldots, n\})$. That is, the float precision is always feasible, but for the given failure rate constraint the probability that the ambiguities can be resolved slowly increases until epoch 54 , from which on the fixed solution can always be accepted. This is illustrated with the gray regions of different shade, where the darkest gray corresponds to $100 \%$, the lightest gray to $5 \%$, and the other three shades are spaced linearly in between. For data-driven partial ambiguity resolution, the index set $\mathcal{I}$ can generally assume different values, leading to precision values other the only the float and fully fixed precision that are obtained with the probabilities $P(\mathcal{I}=\mathcal{J}), \mathcal{J} \in \mathfrak{I}$. After 54 epochs, again all $n$ dimensional integer solutions can be accepted.

The second column shows the ambiguity reliability at which a precision of $\alpha \leq 10$ can be obtained over time. This precision is reached by the float solution after 35 epochs as indicated by the orange line. The fully fixed precision is always below 10 , so the green line shows the reliability of simply resolving the full set of ambiguities without further reliability considerations. This directly explains model-driven full ambiguity resolution. After 35 epochs the given precision can be reached with a failure rate of zero, but before that the failure rate is equal to the failure rate of full ambiguity resolution as defined by the strength of the system model. For model-driven 

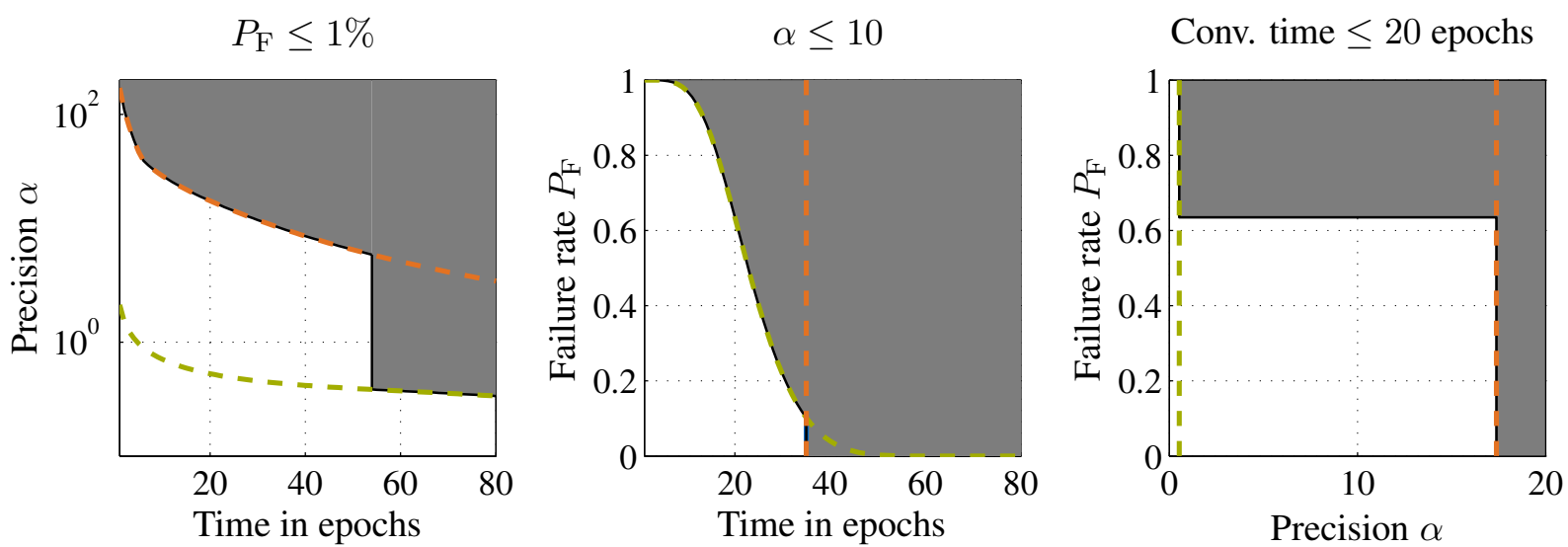

(a) Model-driven full ambiguity resolution
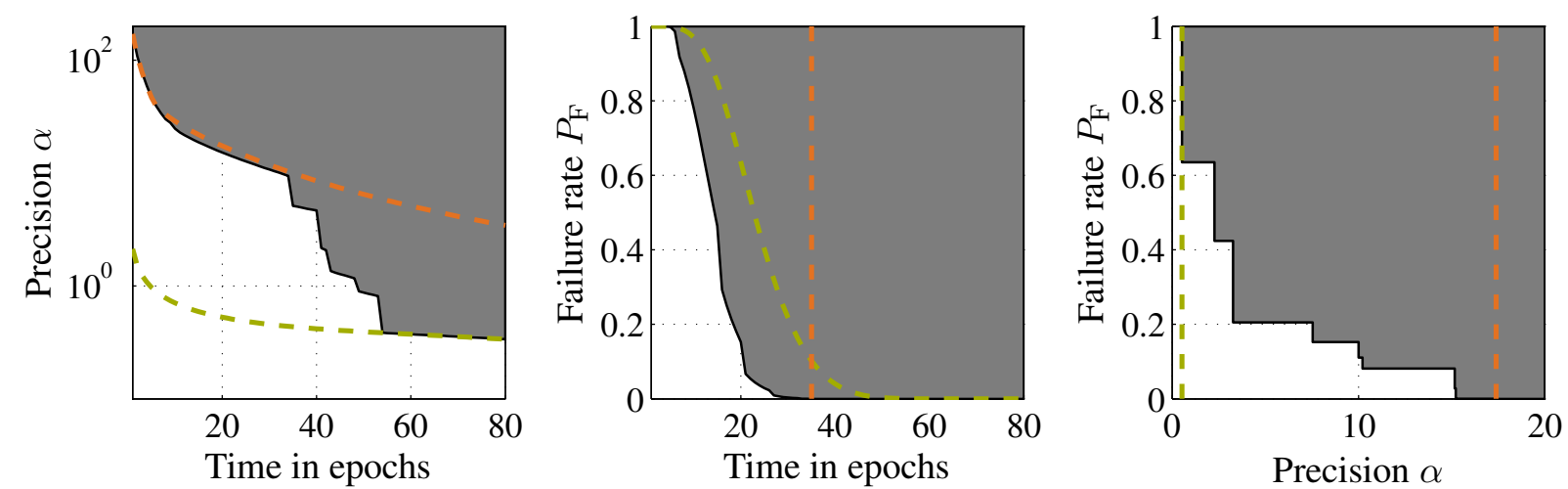

(b) Model-driven partial ambiguity resolution
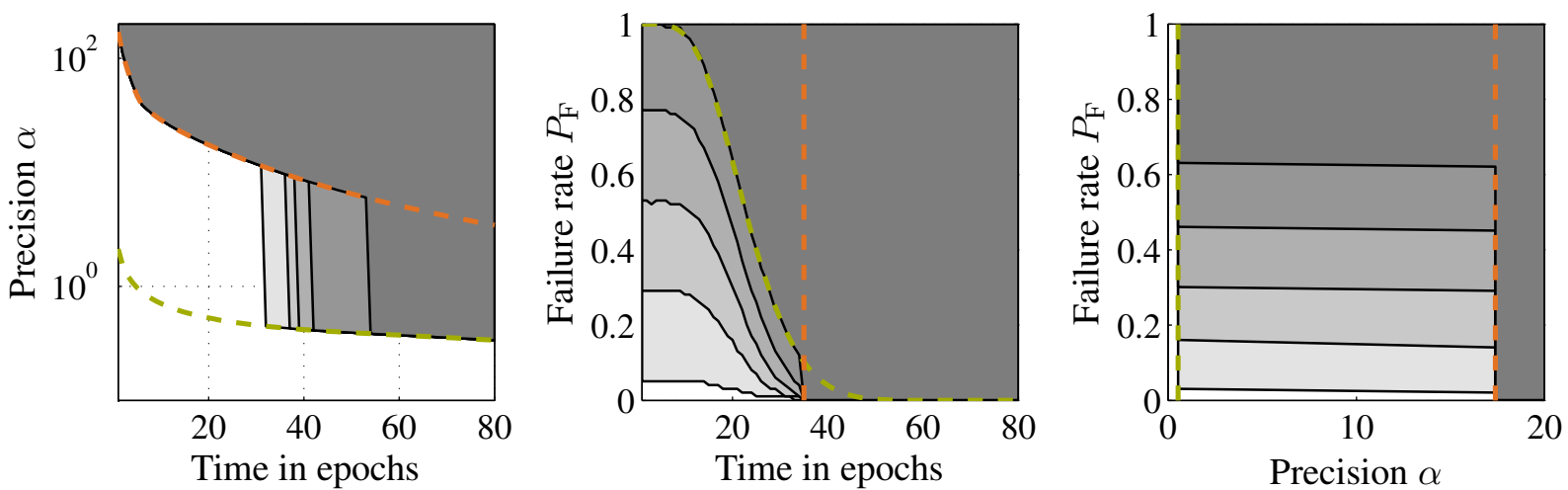

(c) Data-driven full ambiguity resolution
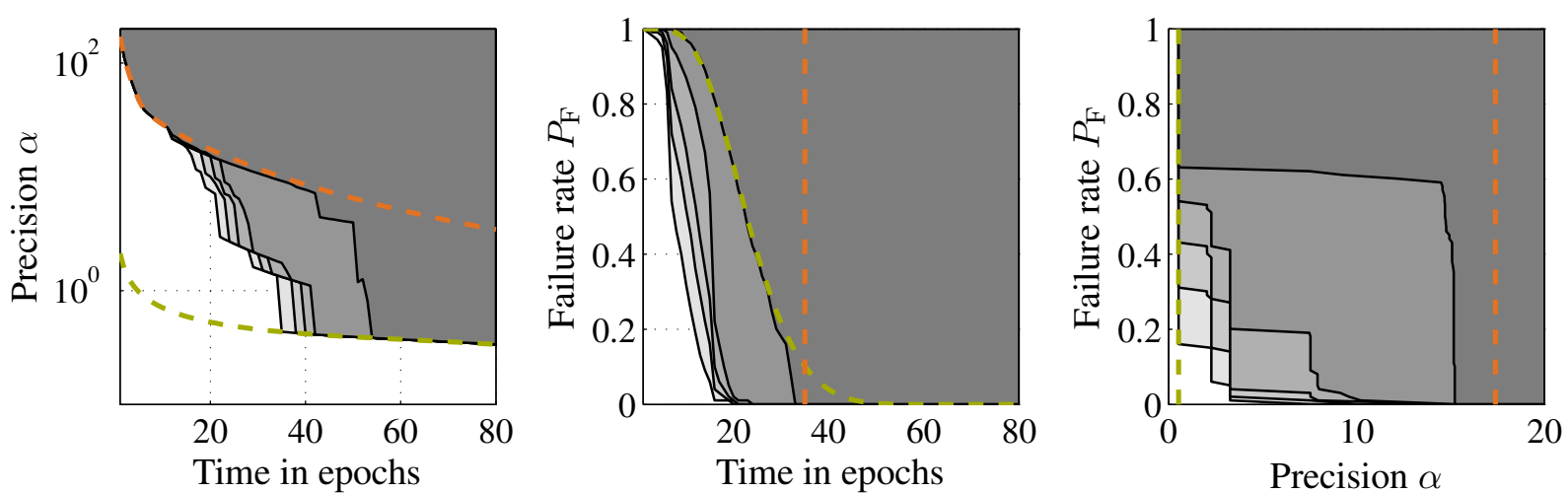

(d) Data-driven partial ambiguity resolution

Figure 6.2: Feasible combinations of the three performance measures time, precision $\alpha$, and failure rate $P_{\mathrm{F}}$ are marked in gray; for the data-driven schemes, different shades correspond to different probabilities (see text); the orange and green dashed lines represent the float and fully fixed solution. 
partial ambiguity resolution, resolving a true subset of the ambiguities might be sufficient to reach $\alpha \leq 10$, which is generally possible with a lower failure rate. The different regions of the datadriven schemes show that if an integer solution is only accepted with a certain probability, then the (partially) ambiguity fixed precision is also only reached with that probability, but the failure rate is smaller than the one indicated by the green line. It is noted that in the first epochs the boundaries of the regions of data-driven full ambiguity resolution are essentially identical to the probability that they represent. That is, the fixed precision value can for instance be reached with a probability of $80 \%$, if one can also accept a failure rate of $80 \%$.

The right column shows the ambiguity reliability at which different precision values can be reached after 20 epochs. The orange and green lines correspond to the float and fully fixed precision. The float precision can always be reached with zero failure rate. With model-driven full ambiguity resolution, the model allows for an ambiguity failure rate of $P_{\mathrm{F}}=63.5 \%$, which defines how reliably the fully fixed precision can be reached. With model-driven partial ambiguity resolution, smaller failure rates can be obtained when resolving different subsets of ambiguities, which correspond to precision values somewhere between the float and fully fixed precision. With the data-driven schemes, the probability of resolving the full set or certain subsets of the ambiguities can be controlled, which also controls the probabilities of obtaining (partially) fixed precision values and implicitly also the failure rate.

Table 6.1: Subset selection strategies for reliable full ambiguity resolution (FAR) and partial ambiguity resolution (PAR) with a maximum failure rate constraint.

\begin{tabular}{|c|c|c|}
\hline & Abbreviation & Acceptance criterion, subset selection strategy \\
\hline \multirow[t]{2}{*}{ Model-driven FAR } & ILS FAR & $P_{\mathrm{F}, \mathrm{ILS}}$ from Monte Carlo integration \\
\hline & IB FAR & $P_{\mathrm{F}, \mathrm{IB}}$ from closed form expression \\
\hline \multirow[t]{3}{*}{ Model-driven PAR } & BM PAR & Best subset $\mathcal{I}$ (in terms of $\alpha$ ) for which full integer \\
\hline & & $\begin{array}{l}\text { least-squares + selection meets failure rate constraint, } \\
P_{\mathrm{F}, \text { fullILS }} \text { from Monte Carlo integration }\end{array}$ \\
\hline & IB PAR & $\mathcal{I}$ from truncated integer bootstrapping (4.32) \\
\hline \multirow[t]{3}{*}{ Data-driven FAR } & DT FAR & Difference test (3.32), \\
\hline & & $\mu$ from Monte Carlo integration \\
\hline & DT FAR F & $\begin{array}{l}\text { Difference test ( } 3.32) \text {, } \\
\mu \text { from functional approximation }\end{array}$ \\
\hline \multirow[t]{3}{*}{ Data-driven PAR } & SS DT PAR & $\begin{array}{l}\text { Single-subset difference test }(5.29) \text {, where } \mathcal{I}^{\prime} \text { is chosen } \\
\text { such that } P\left(\mathcal{I}=\mathcal{I}^{\prime}\right) \text { is maximum among all } \mathcal{I}^{\prime} \text { that lead } \\
\text { to a certain value of } \alpha, \mu \text { from Monte Carlo integration }\end{array}$ \\
\hline & DT PAR & $\begin{array}{l}\text { Per element difference test }(5.33) \\
\mu \text { from Monte Carlo integration }\end{array}$ \\
\hline & DT PAR F & $\begin{array}{l}\text { Per element difference test }(5.33), \\
\mu \text { from functional approximation }\end{array}$ \\
\hline
\end{tabular}

In the remainder of this chapter an extensive analysis of model-driven and data-driven full and partial ambiguity resolution for reliable GNSS positioning is presented on the basis of simulated and real data. The employed estimators are listed in Table 6.1. All estimators are formulated such that a maximum failure rate constraint is not exceeded. The estimator BM PAR selects the deterministic subset $\mathcal{I}$ of ambiguities to be resolved as the one that leads to the most precise coordinates 


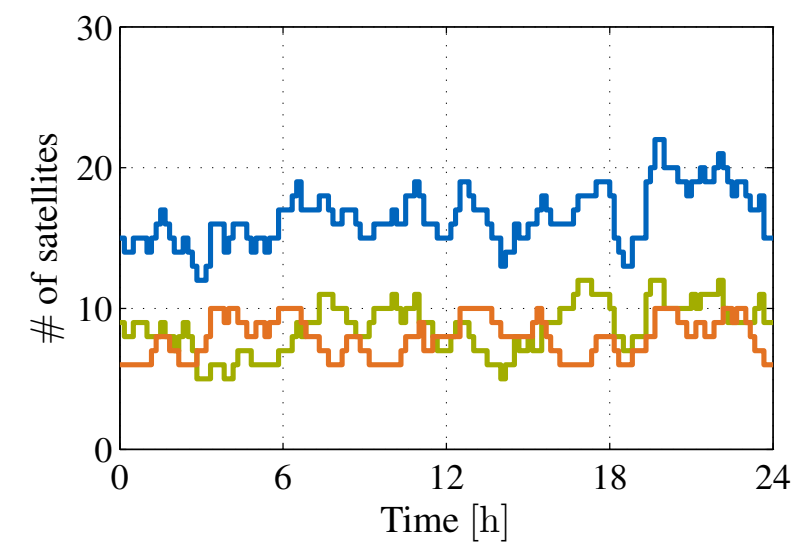

Figure 6.3: Visibility of GPS (green) and Galileo (orange) satellites during one day in Munich, Germany; the number of satellites available for combined GPS+Galileo is shown in blue.

in terms of $\alpha$ among all subsets that meet the failure rate constraint when applying full integer least-squares followed by a selection of entries. For SS DT PAR, we make use of the difference test based single-subset generalized integer aperture estimator, for which $\mathcal{I}^{\prime}$ (see Section 5.3.2) is chosen such that $P\left(\mathcal{I}=\mathcal{I}^{\prime}\right)$ is maximum among all non-empty possible choices for $\mathcal{I}^{\prime}$ for which $\boldsymbol{Q}_{\hat{b} \mid \hat{\boldsymbol{a}}_{\mathcal{I}^{\prime}}}$ meets a certain precision criterion. These two estimators imply a search for the best subset, meaning that many integer subsets $\mathcal{I}$ or $\mathcal{I}^{\prime}$ have to be evaluated. Considering that the number of possible subsets grows exponentially with the number of ambiguities $n$, they are therefore often not a practical choice and should rather be seen as a theoretical benchmark to which the other schemes can be compared. Also, all estimators that are based on Monte Carlo integration entail a rather high computational complexity. The partial ambiguity resolution methods are applied in the LAMBDA decorrelated space, and $P_{\mathrm{F}, \mathrm{IB}}$ for IB FAR is computed after the LAMDBA decorrelation.

\subsection{Numerical Simulations}

The purpose of the simulation based analysis is twofold. First, the capabilities of full and partial ambiguity resolution for reliable GNSS positioning using both model-driven and data-driven methods are investigated and compared. Second, the impact of using low complexity implementations that entail a certain performance degradation is examined. In particular, this means the impact of using integer bootstrapping failure rates as an upper bound for the model-driven schemes and the impact of approximating the true fixed failure rate critical values $\mu$ with a functional description for the difference test based data-driven schemes. Moreover, it also means that the computationally complex exhaustive search for a best integer subset for BM PAR and SS DT PAR is avoided by using truncated integer bootstrapping (IB PAR) and the per element difference test (DT PAR).

The performance study is based on one day of simulated double difference GNSS measurements collected at two receivers in the area of Munich, Germany. We consider single and combined system GPS and/or Galileo cases, where the GPS constellation is used as seen during GPS week 1,815 and Galileo is modeled as Walker Delta $56^{\circ}: 27 / 3 / 1$ constellation (Walker 1984). The number of visible satellites with an elevation angle greater than $10^{\circ}$ is shown in Figure 6.3. The considered signals are listed in Table 6.2. Each satellite is assumed to transmit all three signals. If both systems are combined, a separate pivot satellite is used for each system. The standard deviation of the undifferenced code and carrier-phase observations in Table 6.2 are amplified with the elevation dependent exponential weighting function as explained in Section 2.3. If applicable, the time between two consecutive measurement epochs is $30 \mathrm{~s}$. The presented results are computed in terms of the average performance of the different estimation schemes over the whole day, where 
Table 6.2: Assumed zenith referenced code and carrier-phase standard deviations of GPS and Galileo observations.

\begin{tabular}{lllllll}
\hline & L1 & L2 & L5 & E1 & E5a & E5b \\
\hline$\sigma_{p}[\mathrm{~cm}]$ & 25 & 25 & 20 & 25 & 20 & 20 \\
$\sigma_{\phi}[\mathrm{mm}]$ & 3 & 3 & 3 & 3 & 3 & 3 \\
\hline
\end{tabular}

each scheme is reinitialized every ten minutes. The failure rate constraint is set to $P_{\mathrm{F}} \leq 0.1 \%$. The results are in part also presented in Brack (2016b).

\subsubsection{Short Baselines}

The distance between the two receivers is assumed to be sufficiently short and the receivers are assumed to be at roughly the same height, so that differential atmospheric delays are negligible and the atmosphere fixed model can be used. The user coordinates are assumed completely unlinked from one epoch to the next, and the integer ambiguities are time constant. Reliable instantaneous ambiguity resolution is mostly feasible on short baselines when using measurements on two or three frequencies and also when combining multiple GNSS on only one frequency. We therefore focus on single frequency, single system cases, presented for the example of Galileo E1.

In Figure 6.4, the average probabilities for obtaining precise coordinate estimates with $\alpha \leq 2$ and $\alpha \leq 10$, cf. (6.1), are shown as a function of the number of visible satellites and the number of epochs for each a Monte Carlo based model-driven and data-driven estimator for full and partial ambiguity resolution. For $\alpha \leq 2$, i.e., a standard deviation of $2 \mathrm{~cm}$ in the horizontal plane and $6 \mathrm{~cm}$ for the up component, we can see a clear gain in probability of the data-driven schemes over the model-driven schemes, but no gain of partial ambiguity resolution over full ambiguity resolution. If, however, the precision criterion is relaxed to $\alpha \leq 10$, i.e., to decimeter level precision in the horizontal plane, partial ambiguity resolution indeed becomes useful, especially for a small number of satellites. The data-driven SS DT PAR works slightly better than the model-driven BM PAR. The performance with full ambiguity resolution is unchanged with respect to $\alpha \leq 2$, which means that ambiguity resolution is still required in order to reach $\alpha \leq 10$.

Comparing Figure 6.5 to Figure 6.4 shows the impact of using the easy-to-compute failure rate of (truncated) integer bootstrapping to select the ambiguity subset to be resolved for the modeldriven schemes, and the impact of using the functional approximation for the critical value for the data-driven schemes, for DT PAR F in combination with the implicit subset selection through the per element acceptance test. By close inspection the results of the low complexity estimators in Figure 6.5 can be seen to be slightly worse, even though the difference is hardly noticeable.

\subsubsection{Medium-Length Baselines}

Differential ionospheric delays become relevant once the length of the baseline exceeds a few kilometers. As long as the ionospheric slant delays at both receivers are sufficiently correlated, the difference between them can be modeled as a zero mean Gaussian random variable, i.e., the ionosphere weighted model can be used. In accordance with Odijk (2000), we use the relation

$$
\sigma_{I}=\sqrt{2} \cdot 0.4 \frac{\mathrm{mm}}{\mathrm{km}}
$$

for the distance dependent between receiver differential ionospheric zenith delay on L1 and E1, to which an elevation dependent weighting function is applied, see Section 2.5.3. We focus on instan- 


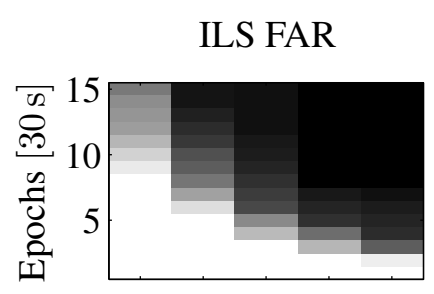

DT FAR

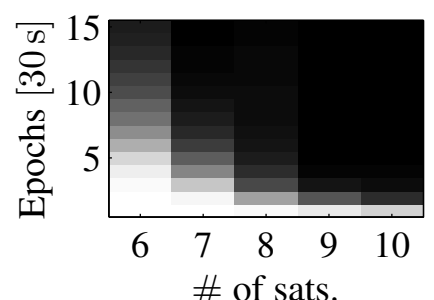

(a) Precision $\alpha \leq 2$
BM PAR

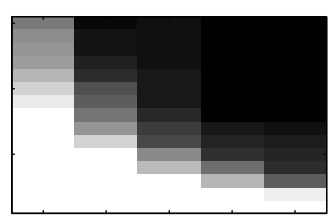

SS DT PAR

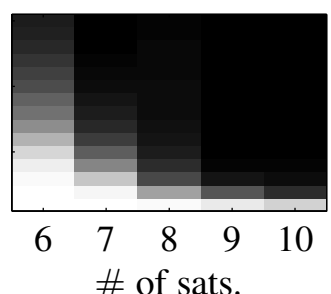

ILS FAR

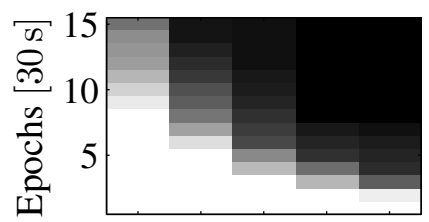

DT FAR

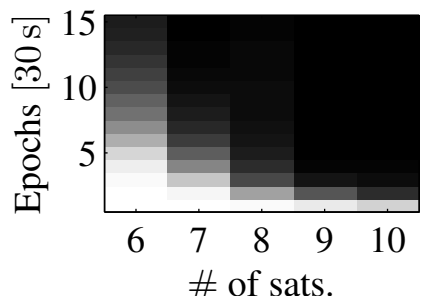

BM PAR

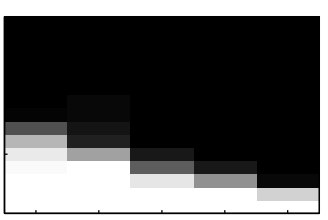

SS DT PAR

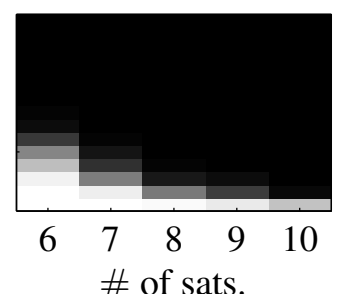

(b) Precision $\alpha \leq 10$

Figure 6.4: Short baseline Galileo E1 RTK positioning with $P_{\mathrm{F}} \leq 0.1 \%$; the estimators are based on Monte Carlo integration combined with an exhaustive search for possible ambiguity subsets for partial ambiguity resolution; white represents an average availability of zero and black an average availability of one.

IB FAR

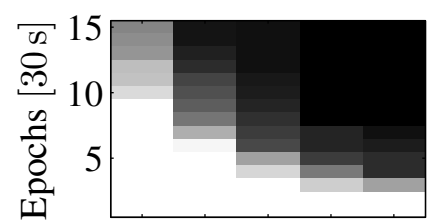

DT FAR F

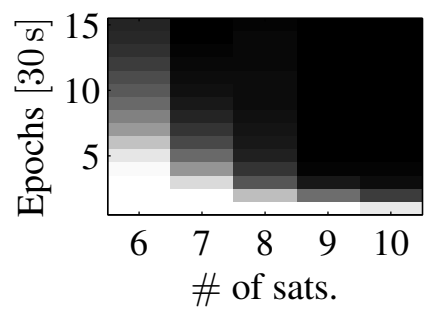

(a) Precison $\alpha \leq 2$
IB PAR

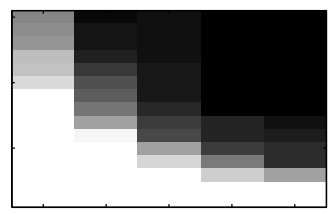

DT PAR F

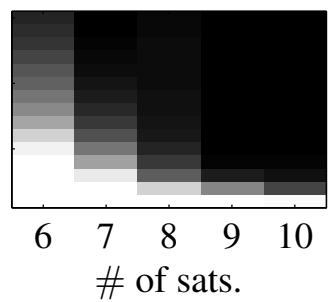

\# of sats.
IB FAR

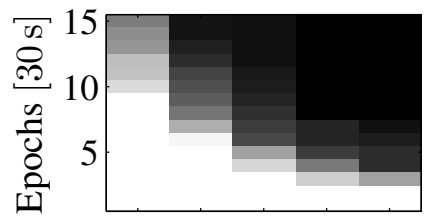

DT FAR F

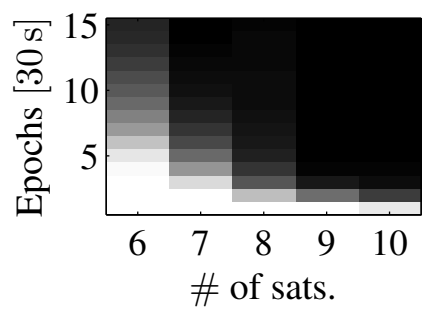

IB PAR

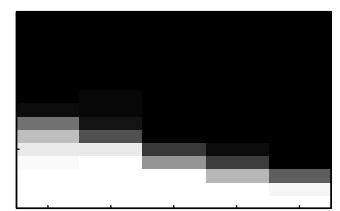

DT PAR F

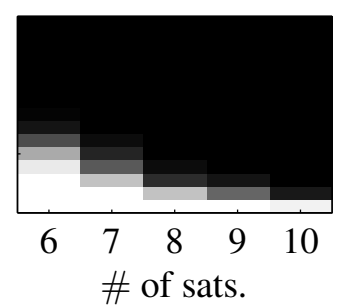

(b) Precision $\alpha \leq 10$

Figure 6.5: Short baseline Galileo E1 RTK positioning with $P_{\mathrm{F}} \leq 0.1 \%$; the estimators are based on low complexity formulations; white represents an average availability of zero and black an average availability of one. 

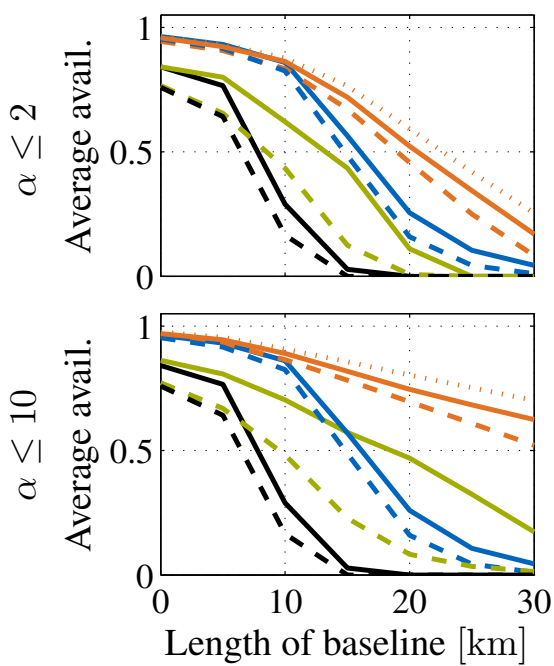

(a) GPS L1+L2
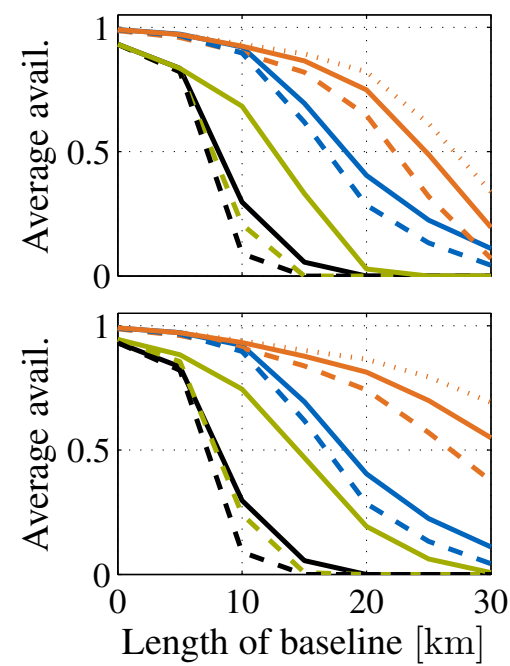

(b) Galileo E1+E5a
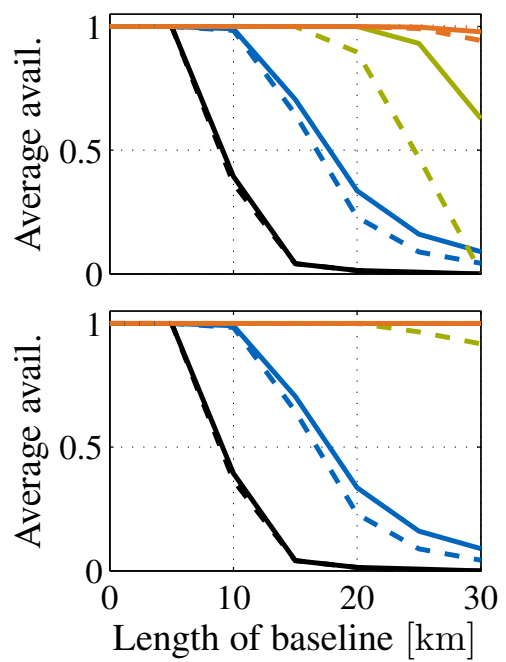

(c) GPS L1+L2 and Galileo $\mathrm{E} 1+\mathrm{E} 5 \mathrm{a}$

Figure 6.6: Dual frequency instantaneous RTK positioning with $P_{\mathrm{F}} \leq 0.1 \%$; black: ILS FAR (solid) and IB FAR (dashed), green: BM PAR (solid) and IB PAR (dashed), blue: DT FAR (solid) and DT FAR F (dashed), orange: SS DT PAR (dotted) and DT PAR (solid) and DT PAR F (dashed).

taneous RTK positioning using only a single epoch of observations from dual and triple frequency receivers. Differential tropospheric delays are assumed absent in the following simulations.

Figure 6.6 shows the average probabilities for obtaining precise and reliable coordinate estimates for dual frequency receivers, where precise means $\alpha \leq 2$ in the first row and $\alpha \leq 10$ in the second row. Both for single and combined systems, this probability drops very soon for the model-driven full ambiguity resolution schemes when the baseline exceeds a few kilometers, as is shown with the black lines. The dashed black line shows the impact of using $P_{\mathrm{F}, \mathrm{IB}}$ as an upper bound for $P_{\mathrm{F}, \mathrm{ILS}}$ (solid black line) when deciding about whether or not to resolve the full set of ambiguities. Better results are obtained with the model-driven partial ambiguity resolution schemes, shown in green, especially for the combined GPS+Galileo setup. We can also see that the very simple truncated bootstrapping subset selection scheme (IB PAR, dashed green) can be clearly worse than evaluating all possible subsets (BM PAR, solid green). The data-driven difference test based schemes lead to much higher probabilities, where partial ambiguity resolution, shown in orange, is again clearly superior to full ambiguity resolution as shown in blue, in particular for longer baselines. The solid lines represent the (per element) difference test with the true fixed failure rate critical values and the dashed lines are based on the functional approximation. Especially for the high availability regime, which is the aimed at operating point of a positioning system, the impact of the approximation is very small. Finally, the dotted orange lines represent SS DT PAR. Although this estimator chooses the subset $\mathcal{I}^{\prime}$ in $\mathfrak{I}=\left\{\emptyset, \mathcal{I}^{\prime}\right\}$ such that the availability for the given $\alpha$ is maximized, its performance is only slightly better than the-computationally much simplerper element difference test for both considered values of $\alpha$. For the combined GPS+Galileo setup, instantaneous RTK positioning is basically always possible with the per element difference test for baselines of at least up to $30 \mathrm{~km}$. The partial ambiguity resolution schemes show better results for a more relaxed value of $\alpha$. This demonstrates the fundamental principle of partial ambiguity resolution, i.e., the precision of the position estimates may be not as good as after full ambiguity resolution, but the availability can be clearly increased. The corresponding results for signals on 

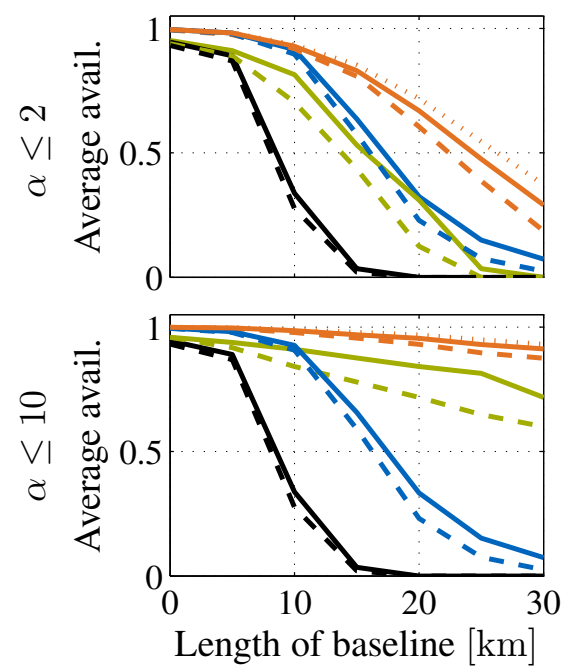

(a) GPS L1+L2+L5
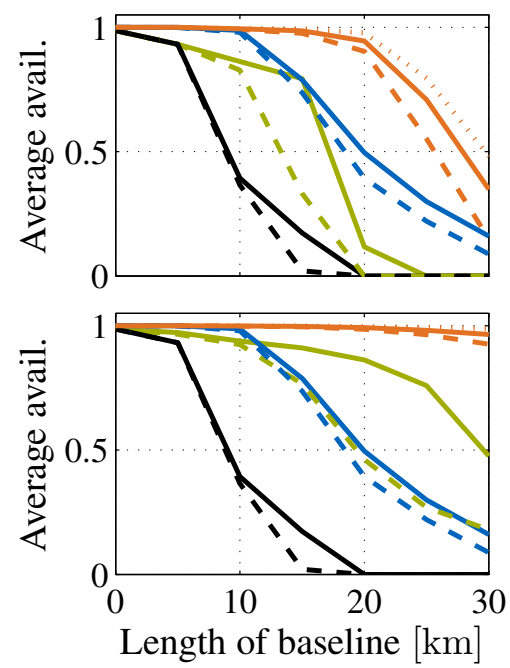

(b) Galileo E1+E5a+E5b
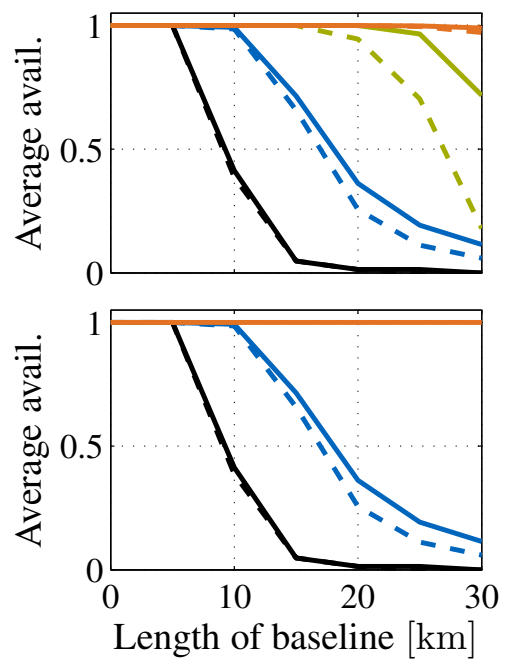

(c) GPS $\mathrm{L} 1+\mathrm{L} 2+\mathrm{L} 5$ and Galileo $\mathrm{E} 1+\mathrm{E} 5 \mathrm{a}+\mathrm{E} 5 \mathrm{~b}$

Figure 6.7: Triple frequency instantaneous RTK positioning with $P_{\mathrm{F}} \leq 0.1 \%$; black: ILS FAR (solid) and IB FAR (dashed), green: BM PAR (solid) and IB PAR (dashed), blue: DT FAR (solid) and DT FAR F (dashed), orange: SS DT PAR (dotted) and DT PAR (solid) and DT PAR F (dashed).

three frequencies for each system are presented in Figure 6.7. Due to the increased strength of the underlying system models, the performance of all schemes is somewhat enhanced.

In summary, the results indicate that with partial ambiguity resolution techniques we can expect a clearly increased range of instantaneous ambiguity resolution enabled centimeter level positioning. The results are based on the ionosphere weighted model. This model can not only be used for single baseline positioning, but also for many network based positioning applications, where ionospheric corrections are interpolated to the approximate user position and provided together with their probabilistic description. Such ionospheric corrections are for instance essential for fast positioning results with network RTK or PPP-RTK. Partial ambiguity resolution techniques should therefore also be beneficial for loosening the quality requirements of these corrections, e.g., to allow for a less dense network of reference stations, see also Section 6.2.4. Furthermore, the simulations show that partial ambiguity resolution proves particularly useful for future multi-GNSS solutions.

\subsubsection{Long Baselines}

With long baseline it is referred to the case that the spatial correlation between the atmospheric delays experienced by the signals observed at the two receivers is too weak to be properly utilized. The residual between receiver differential tropospheric zenith wet delay and the double difference ionospheric slant delays are thus included as unknowns in the parameter vector $\boldsymbol{b}$. We assume that user coordinates, ambiguities, and the residual tropospheric zenith delay are time constant, whereas the ionospheric delays are completely unlinked in time. We define the convergence time as the number of epochs required with ILS FAR or IB FAR to reach a formal precision of $\alpha \leq 2$ or $\alpha \leq 10$. It is important to note that the so defined convergence time is not necessarily identical to the time required to fix the ambiguities. The average convergence times are given in Table 6.3 for different available signals. The smaller values for $\alpha \leq 10$ imply that this precision may already 
Table 6.3: Average convergence times of ILS FAR (in parentheses IB FAR) in terms of the required number of epochs; one epoch corresponds to $30 \mathrm{~s}$.

\begin{tabular}{lll}
\hline & $\alpha \leq 2$ & $\alpha \leq 10$ \\
\hline GPS L1+L2 & $56.0(58.7)$ & $27.7(27.7)$ \\
GPS L1+L2+L5 & $39.5(41.1)$ & $24.2(24.2)$ \\
Galileo E1+E5a & $59.4(61.0)$ & $34.5(34.6)$ \\
Galileo E1+E5a+E5b & $47.8(49.5)$ & $33.5(33.7)$ \\
GPS L1+L2+ Galileo E1+E5a & $42.9(44.2)$ & $17.9(18.0)$ \\
GPS L1+L2+L5 + Galileo E1+E5a+E5b & $19.2(20.2)$ & $14.8(15.4)$ \\
\hline
\end{tabular}
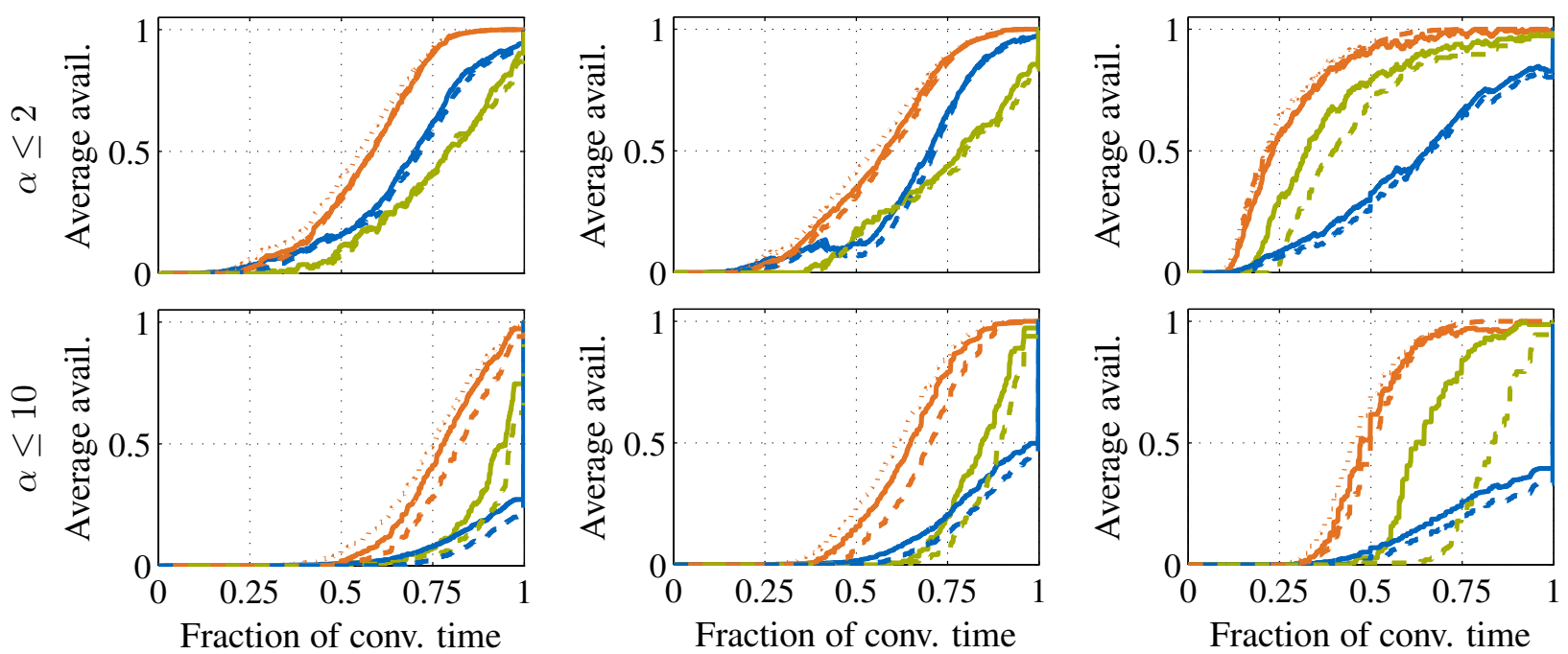

(a) GPS L1+L2

(b) Galileo E1+E5a

(c) GPS L1+L2 and Galileo $\mathrm{E} 1+\mathrm{E} 5 \mathrm{a}$

Figure 6.8: Dual frequency long baseline positioning with $P_{\mathrm{F}} \leq 0.1 \%$; green: BM PAR (solid) and IB PAR (dashed), blue: DT FAR (solid) and DT FAR F (dashed), orange: SS DT PAR (dotted) and DT PAR (solid) and DT PAR F (dashed).

be reached with the float solution. Improved convergence times are obtained with the combined system.

The model-driven full ambiguity resolution schemes are conceptually always the weakest schemes, meaning that if $P_{\mathrm{F}, \mathrm{ILS}} \leq 0.1 \%$, so that the full integer solution can be accepted with ILS FAR, then also BM PAR results in resolving the full set of ambiguities and the difference test based schemes DT FAR, SS DT PAR, and DT PAR resolve the full set of ambiguities with probability one. Similarly, we have for the low complexity schemes that if $P_{\mathrm{F}, \mathrm{IB}} \leq 0.1 \%$, then also IB PAR resolves the full set of ambiguities and the critical values as produced by the functional descriptions used with DT FAR F and DT PAR F are such that the full set of ambiguities is resolved with probability one. We can therefore investigate by how much the convergence times as defined above can be expected to be reduced on average by using one of the more advanced ambiguity resolution schemes.

Figure 6.8 shows the average availability of precise and reliable coordinate estimates for dual frequency receivers as a function of the fraction of the convergence time of ILS FAR for the Monte Carlo based estimators (solid and dotted lines) and of IB FAR for the ones that are based on the 

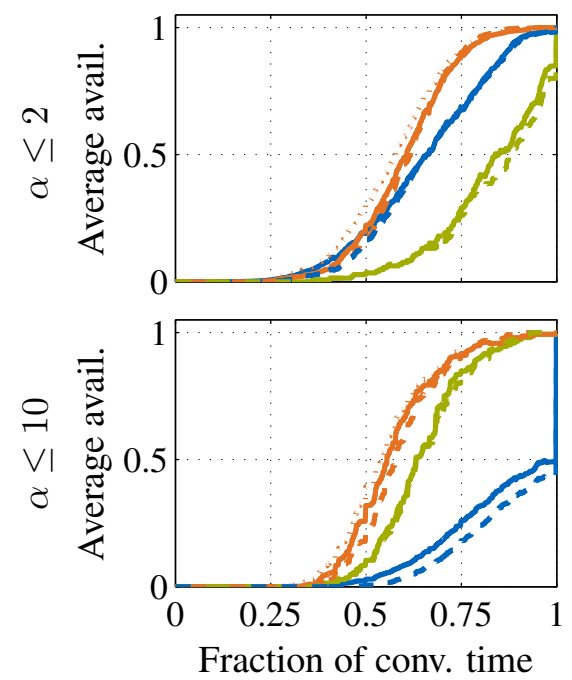

(a) GPS L1+L2+L5
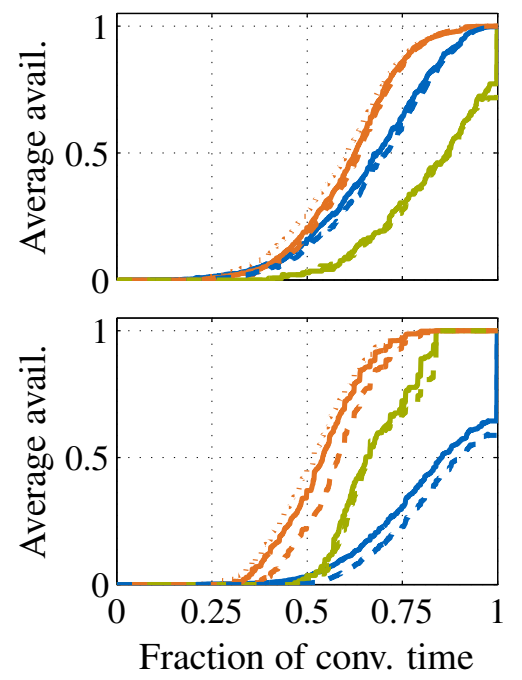

(b) Galileo E1+E5a+E5b
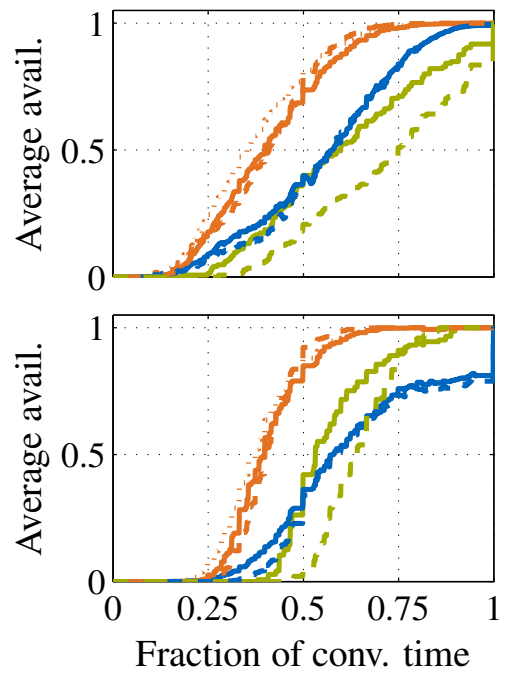

(c) GPS L1+L2+L5 and Galileo $\mathrm{E} 1+\mathrm{E} 5 \mathrm{a}+\mathrm{E} 5 \mathrm{~b}$

Figure 6.9: Triple frequency long baseline positioning with $P_{\mathrm{F}} \leq 0.1 \%$; green: BM PAR (solid) and IB PAR (dashed), blue: DT FAR (solid) and DT FAR F (dashed), orange: SS DT PAR (dotted) and DT PAR (solid) and DT PAR F (dashed).

integer bootstrapping failure rate (dashed lines). The difference between the solid and dashed blue and orange lines can therefore not directly be interpreted as the impact of using the functional approximation for the critical values for the (per element) difference test. Again, partial ambiguity resolution is particularly useful for combined GPS+Galileo, where for $\alpha \leq 2$ the data-driven partial ambiguity resolution schemes, shown in orange, can on average reduce the convergence time by $75 \%$ with a probability of more than $50 \%$ and by half with a probability of more than $90 \%$. For this case, also the simpler model-driven partial ambiguity resolution schemes in green show very good yet slightly inferior performance. The smaller gains of all estimators for $\alpha \leq 10$ are caused by the fact that after a certain number of epochs already the float solution may reach this precision, in which case all estimators are equally useful when only considering such a binary performance criterion. This becomes clear if we look at the average availability of DT FAR and DT FAR F, which do not slowly converge to $100 \%$ over time but rather have a step at a relative time of one. The corresponding results for triple frequency receivers are presented in Figure 6.9.

\subsubsection{Multiple Baselines}

In a network RTK scenario with multiple nearby reference stations, the user receiver can form multiple baselines simultaneously and jointly estimate the involved parameters, which are the user position, the carrier-phase ambiguities from all baselines, and optionally differential atmospheric delays. We assume that the measurements at the reference stations are a priori corrected for the exact atmospheric delays and that the user receiver is provided with estimates for the atmospheric delays at its approximate position in each epoch, which are applied by means of the atmosphere weighted model. We assume a standard deviation of the ionospheric corrections of $2 \mathrm{~cm}$ in zenith direction, to which the elevation dependent exponential weighting function is applied. The tropospheric correction is assumed to be exact. The user coordinates are assumed completely unlinked in time. 

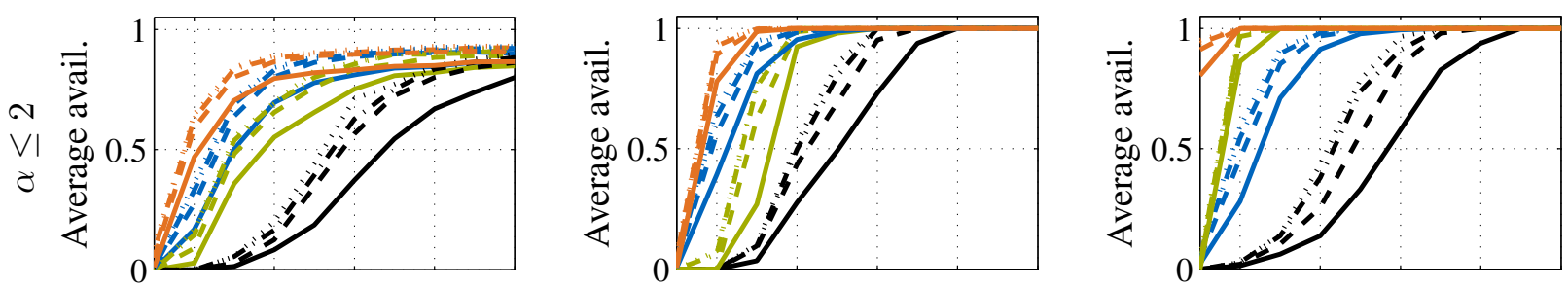

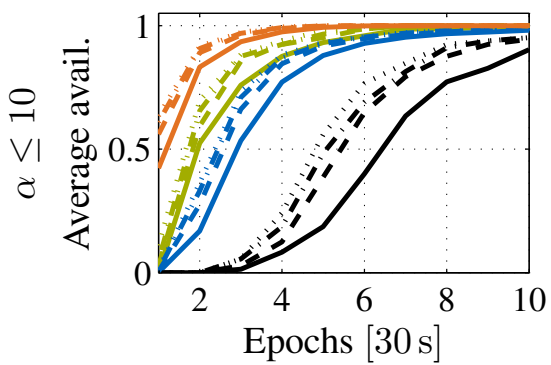

(a) GPS L1+L2

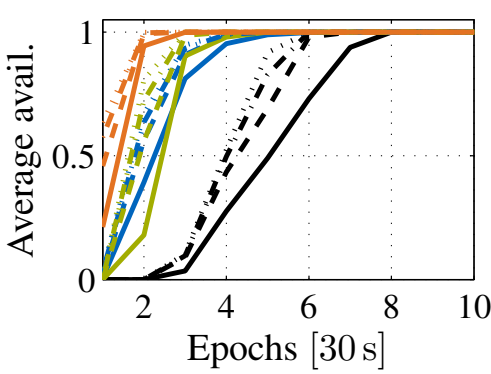

(b) Galileo E1+E5a

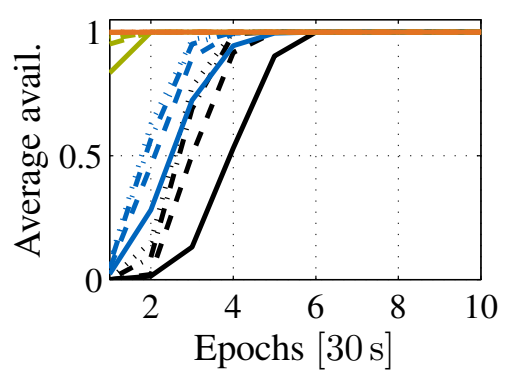

(c) GPS L1+L2 and Galileo $\mathrm{E} 1+\mathrm{E} 5 \mathrm{a}$

Figure 6.10: Dual frequency positioning with multiple baselines and $P_{\mathrm{F}} \leq 0.1 \%$; black: IB FAR, green: IB PAR, blue: DT FAR F, orange: DT PAR F; solid/dashed/dash-dotted/dotted correspond to 1/2/3/4 baselines.

Figure 6.10 shows the average probabilities for obtaining precise and reliable estimates of the user coordinates as a function of the number of epochs, where solid, dashed, dash-dotted, and dotted lines correspond to one, two, three, and four baselines, respectively. Comparing the results of the model-driven full and partial ambiguity resolution schemes IB FAR (black) and IB PAR (green) and the data-driven DT FAR F (blue) and DT PAR F (orange) confirms what could be expected from the previous results. With model-driven full ambiguity resolution, it takes on average quite many epochs until a satisfactory precision is reached. This time can be shortened with model-driven partial ambiguity resolution, and the data-driven schemes again clearly outperform their model-driven counterparts. Partial ambiguity resolution is particularly beneficial for the combined system, where mostly not more than one or two epochs are necessary in order to reach $\alpha \leq 2$ or $\alpha \leq 10$. These observations hold for all considered numbers of baselines. All four estimators show a comparable performance gain with increasing number of baselines.

\subsection{Real-Data Analysis}

From the simulation results we can expect partial ambiguity resolution to be very useful for reliable positioning on medium-length or long baselines, in particular for a combined multi-GNSS solution. These results are now verified with $24 \mathrm{~h}$ of real GPS and BDS data, which was collected during September 7, 2015, at the IGS MGEX stations CUT0, PERT, and NNOR in the area of Perth, Australia. The time between two consecutive measurements is $30 \mathrm{~s}$. We consider instantaneous dual frequency RTK positioning on the $22.4 \mathrm{~km}$ baseline between CUT0 and PERT, which are both equipped with geodetic Trimble NetR9 receivers, and dual frequency long baseline positioning on the $88.5 \mathrm{~km}$ baseline between PERT and NNOR, which is equipped with a Septentrio PolaRx 4 receiver. The number of satellites that is jointly visible at both receivers with an elevation angle greater than $10^{\circ}$ is shown in Figure 6.11 for both baselines. The long baseline often results in slightly fewer available satellites. The considered signals and their zenith referenced code and carrier-phase standard deviations are listed in Table 6.4. The code standard deviations were es- 


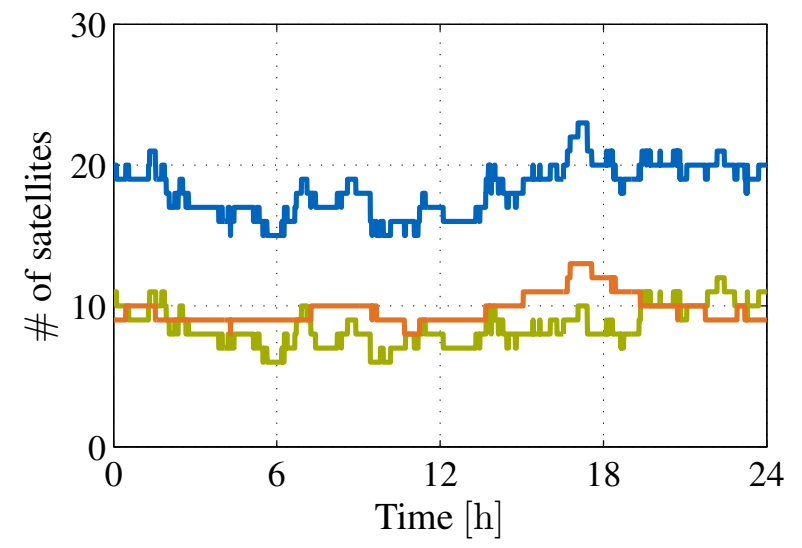

(a) $22.4 \mathrm{~km}$ baseline CUT0-PERT

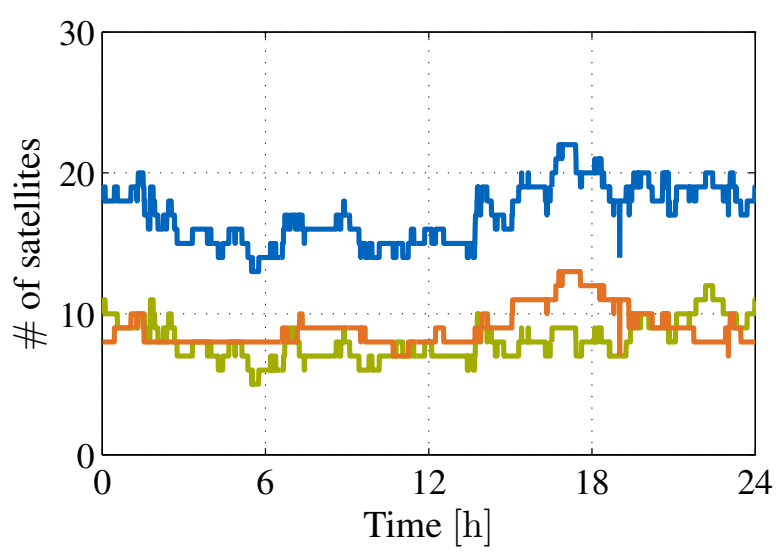

(b) $88.5 \mathrm{~km}$ baseline PERT-NNOR

Figure 6.11: Visibility of GPS (green) and BDS (orange) satellites in the area of Perth, Australia, during September 7, 2015; the number of satellites available for combined GPS+BDS is shown in blue.

Table 6.4: Estimated zenith referenced code and carrier-phase standard deviations of GPS and BDS observations.

\begin{tabular}{lllll}
\hline & L1 & L2 & B1 & B2 \\
\hline$\sigma_{p}[\mathrm{~cm}]$ & 37 & 28 & 35 & 28 \\
$\sigma_{\phi}[\mathrm{mm}]$ & 2 & 2 & 2 & 2 \\
\hline
\end{tabular}

timated from one day of double difference measurements from the stations CUT0 and CUTA as described in Section 6.3.1. They are different from the ones assumed in Table 6.2 in the previous section. A separate pivot satellite is used for GPS and BDS. Only the four low complexity implementations IB FAR, IB PAR, DT FAR F, and DT PAR F are investigated, where the partial ambiguity resolution techniques are again applied in the LAMBDA decorrelated space and the computation of $P_{\mathrm{F}, \mathrm{IB}}$ for IB FAR is also carried out after the LAMBDA decorrelation. For IB FAR and IB PAR, the (truncated) bootstrapping failure rate is used to evaluate which subset of the ambiguities is resolved, but the estimation of the integers itself is done via full dimensional integer least-squares, for IB PAR followed by a selection of entries. The failure rate constraint is set to $P_{\mathrm{F}} \leq 0.1 \%$.

\subsubsection{Variance Component Estimation}

A prerequisite for the failure rates and the computed formal precision measures to reflect the physical reality are realistic assumptions for the stochastic system model. With the stochastic system models from Section 2.3, we therefore have to determine realistic values for the zenith referenced code and carrier-phase standard deviations $\sigma_{p_{f}}$ and $\sigma_{\phi_{f}}$ for all frequencies $f=1, \ldots, F$ per system. To this end, we consider a linear model of the form

$$
\boldsymbol{y}(t)=\boldsymbol{A}(t) \boldsymbol{x}(t)+\boldsymbol{\eta}(t) \quad \text { with } \quad \boldsymbol{\eta}(t) \sim \mathcal{G}\left(\mathbf{0}, \sigma^{2} \boldsymbol{G}(t)\right)
$$

where $\boldsymbol{y}(t) \in \mathbb{R}^{q_{t}}$ is the measurement vector at time instance $t \in\left\{t_{1}, \ldots, t_{T}\right\}$. The design matrix $\boldsymbol{A}(t)$ and the parameter vector $\boldsymbol{x}(t)$ can be completely different in each epoch. the noise vector $\boldsymbol{\eta}(t)$ is assumed to be the product of the known time dependent factor $\boldsymbol{G}(t) \in \mathbb{R}^{q_{t} \times q_{t}}$ and the unknown but constant $\sigma^{2}$. The noise vector $\boldsymbol{\eta}(t)$ is assumed to be temporally uncorrelated and the 
parameter vectors $\boldsymbol{x}(t), t=t_{1}, \ldots, t_{T}$, are assumed to be known. The unknown variance $\sigma^{2}$ should be estimated based on the measurements from all epochs. With the likelihood function

$$
L\left(\boldsymbol{y}\left(t_{1}\right), \ldots, \boldsymbol{y}\left(t_{T}\right) ; \theta^{2}\right)=\frac{1}{\prod_{t=t_{1}}^{t_{T}} \sqrt{\operatorname{det}\left(2 \pi \boldsymbol{G}(t) \theta^{2}\right)}} \cdot \exp \left(-\frac{1}{2 \theta^{2}} \sum_{t=t_{1}}^{t_{T}}\|\boldsymbol{y}(t)-\boldsymbol{A}(t) \boldsymbol{x}(t)\|_{\boldsymbol{G}(t)}^{2}\right),
$$

the maximum likelihood estimate $\hat{\sigma}^{2}$ of $\sigma^{2}$ is given by

$$
\hat{\sigma}^{2}=\underset{\theta^{2} \in \mathbb{R}_{+}}{\operatorname{argmax}} L\left(\boldsymbol{y}\left(t_{1}\right), \ldots, \boldsymbol{y}\left(t_{T}\right) ; \theta^{2}\right) .
$$

Since the logarithm is an increasing function, this problem can equivalently be solved with the log-likelihood function

$$
\begin{aligned}
l\left(\boldsymbol{y}\left(t_{1}\right), \ldots, \boldsymbol{y}\left(t_{T}\right) ; \theta^{2}\right)= & \log \left(L\left(\boldsymbol{y}\left(t_{1}\right), \ldots, \boldsymbol{y}\left(t_{T}\right) ; \theta^{2}\right)\right) \\
= & -\frac{1}{2} \sum_{t=t_{1}}^{t_{T}} \log \operatorname{det}(2 \pi \boldsymbol{G}(t))-\frac{1}{2} \sum_{t=t_{1}}^{t_{T}} \log \left(\theta^{2 q_{t}}\right) \\
& -\frac{1}{2 \theta^{2}} \sum_{t=t_{1}}^{t_{T}}\|\boldsymbol{y}(t)-\boldsymbol{A}(t) \boldsymbol{x}(t)\|_{\boldsymbol{G}(t)}^{2},
\end{aligned}
$$

with $q_{t}$ the number of measurements at time $t$. Setting the derivative

$$
\frac{\mathrm{d} l\left(\boldsymbol{y}\left(t_{1}\right), \ldots, \boldsymbol{y}\left(t_{T}\right) ; \theta^{2}\right)}{\mathrm{d} \theta^{2}}=-\frac{1}{2} \sum_{t=t_{1}}^{t_{T}} \frac{q_{t}}{\theta^{2}}+\frac{1}{2 \theta^{4}} \sum_{t=t_{1}}^{t_{T}}\|\boldsymbol{y}(t)-\boldsymbol{A}(t) \boldsymbol{x}(t)\|_{\boldsymbol{G}(t)}^{2}
$$

to zero finally yields the maximum likelihood estimate of the variance factor $\sigma^{2}$ as

$$
\hat{\sigma}^{2}=\frac{1}{\sum_{t=t_{1}}^{t_{T}} q_{t}} \cdot \sum_{t=t_{1}}^{t_{T}}\|\boldsymbol{y}(t)-\boldsymbol{A}(t) \boldsymbol{x}(t)\|_{\boldsymbol{G}(t)}^{2} .
$$

More details about maximum likelihood variance component estimation can be found in Harville (1977).

The GNSS positioning model for a single epoch of double difference measurements as discussed in Chapter 2 is of the form

$$
\boldsymbol{y}=\boldsymbol{A} \boldsymbol{a}+\boldsymbol{B} \boldsymbol{b}+\boldsymbol{\eta} \quad \text { with } \quad \boldsymbol{\eta} \sim \mathcal{G}(\mathbf{0}, \boldsymbol{Q}) .
$$

With the assumptions made in Section 2.3 concerning the correlations between the undifferenced measurements, the double difference covariance matrix $Q$ can be written as

$$
\boldsymbol{Q}=2 \cdot \operatorname{diag}\left(\sigma_{p_{1}}^{2}, \ldots, \sigma_{p_{F}}^{2}, \sigma_{\phi_{1}}^{2}, \ldots, \sigma_{\phi_{F}}^{2}\right) \otimes \boldsymbol{D} \boldsymbol{W} \boldsymbol{D}^{\mathrm{T}},
$$

cf. (2.74). Different from (6.3), we therefore have to estimate $2 F$ variance factors instead of only one. Due to the blockdiagonal structure of $Q$ in (6.10), the likelihood function for estimating all $2 F$ variance factors can be factorized into separate terms for the code and carrier-phase variance on each frequency, which only depend on the part of the measurement vector $y$ containing the 

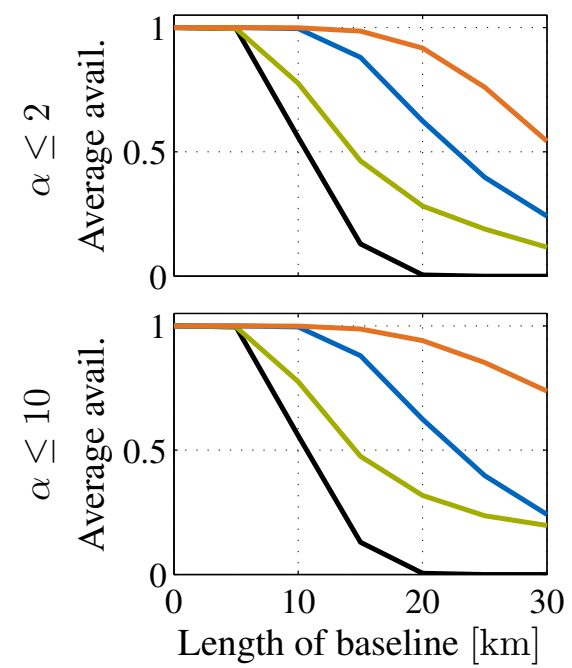

(a) GPS L1+L2
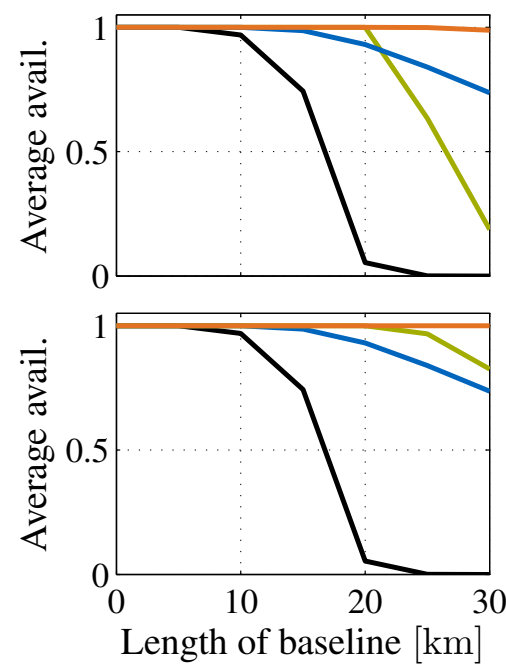

(b) BDS B1+B2
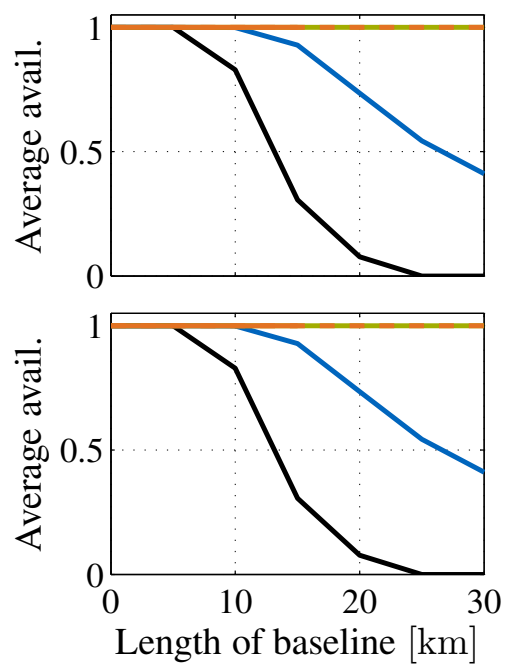

(c) GPS L1+L2 and BDS B1+B2

Figure 6.12: Dual frequency instantaneous RTK positioning with $P_{\mathrm{F}} \leq 0.1 \%$; black: IB FAR, green: IB PAR, blue: DT FAR F, orange: DT PAR F.

respective observations. That is, the joint maximum likelihood estimation of $\sigma_{p_{f}}^{2}$ and $\sigma_{\phi_{f}}^{2}$ with $f=1, \ldots, F$ can be performed separately for each component. With $\boldsymbol{G}=\boldsymbol{D} \boldsymbol{W} \boldsymbol{D}^{\mathrm{T}}$, each of these separate estimation problems fits to the model (6.3). We can now collect a sufficiently large number of measurements, e.g., over a time span of $24 \mathrm{~h}$, and apply (6.8) to each component. This requires the exact knowledge of the parameter vector $\boldsymbol{b}$ for the code variances. This is possible by using measurements from a zero baseline or from two reference stations with precisely known coordinates on a short baseline, so that differential atmospheric delays are safely negligible. For the carrier-phase variances, also the integer ambiguities would have to be a priori known with this approach. More information about variance component estimation for GNSS applications is given in, e.g., Amiri-Simkooei (2007).

\subsubsection{Instantaneous RTK Positioning on CUT0-PERT}

We first analyze the average RTK positioning capabilities by means of the resulting values of the formal precision measure $\alpha$ as a function of the distance between the two receivers via simulations. Independent of the baseline length, the satellite visibility is assumed to be the same as for the real measurements. The ionosphere weighted model is employed, where the zenith referenced standard deviation of the between receiver differential ionospheric delays on L1 and B1 is again modeled depending on the length of the baseline as $\sigma_{I}=\sqrt{2} \cdot 0.4 \frac{\mathrm{mm}}{\mathrm{km}}$. Differential tropospheric delays are assumed absent.

Figure 6.12 shows the average availability of precise and reliable coordinate estimates for $\alpha \leq 2$ and $\alpha \leq 10$, where all 2,880 epochs are considered. The results confirm the findings in Section 6.2.2 for GPS and Galileo and are therefore not discussed in detail. The-compared to GPS and Galileo-improved performance of BDS only positioning is presumably caused by the higher minimum number of visible satellites, see Figures 6.3 and 6.11. If the model assumptions are appropriate, we should hence be able to reach a centimeter level positioning error with partial ambiguity resolution for BDS only and combined GPS+BDS positioning throughout the whole day 


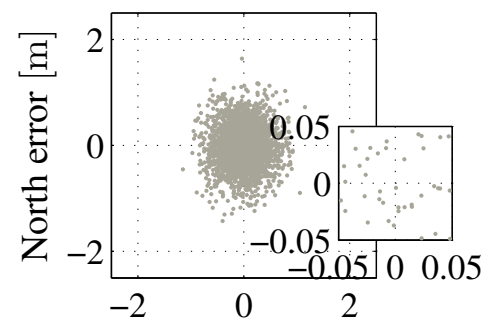

East error $[\mathrm{m}]$
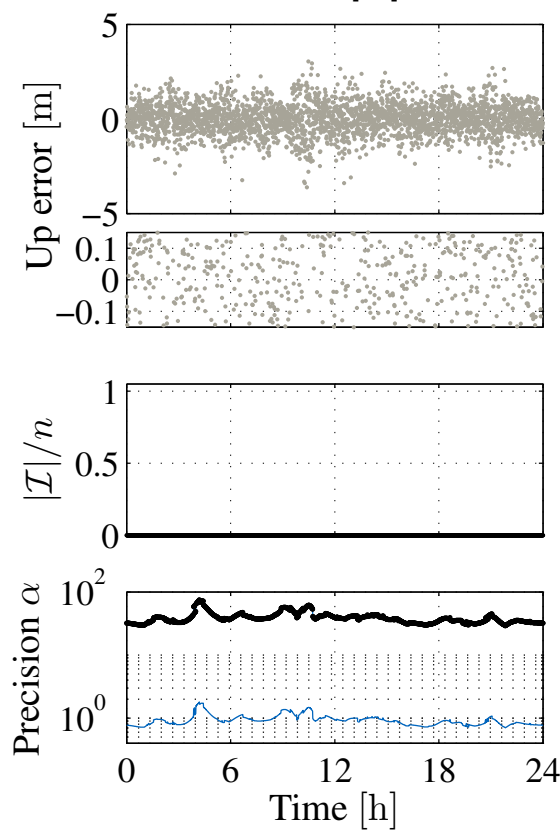

(a) GPS L1+L2

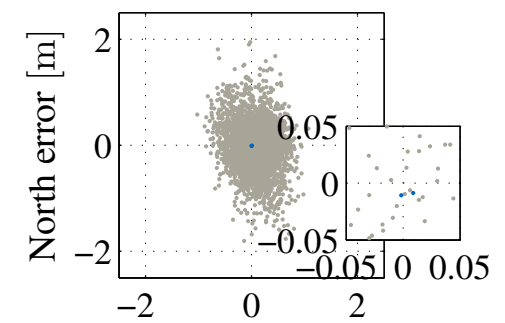

East error $[\mathrm{m}]$
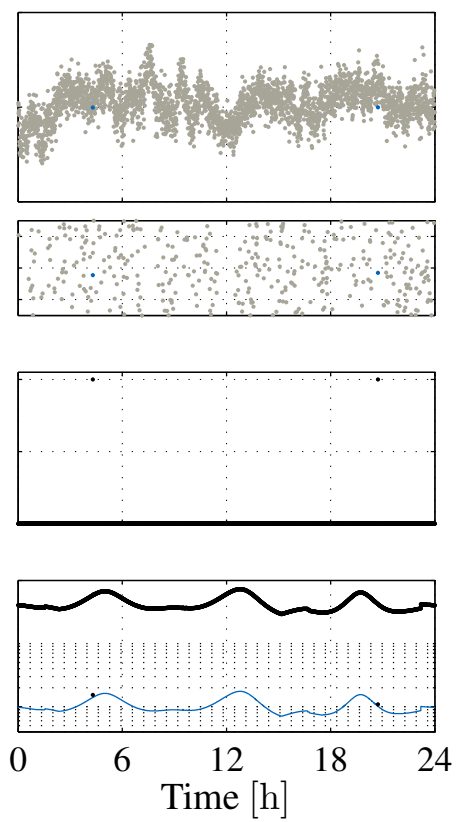

(b) BDS B1+B2

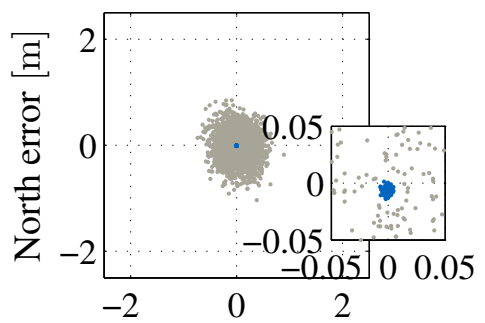

East error $[\mathrm{m}]$
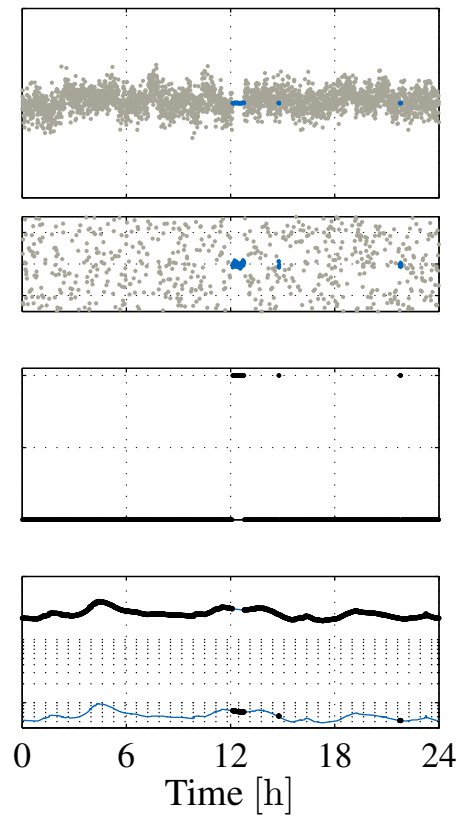

(c) GPS L1+L2 and BDS B1+B2

Figure 6.13: Dual frequency instantaneous RTK positioning results for CUT0-PERT with IB FAR and $P_{\mathrm{F}} \leq 0.1 \%$. The color of the horizontal and vertical components of the positioning error indicates the ratio of fixed ambiguities as shown in the fourth row, where gray stands for a ratio of 0 and blue for a ratio of 1 . In the bottom row, the computed formal precision values $\alpha$ are shown in black. They are limited by the float (upper blue line) and fully fixed (lower blue line) precision.

when analyzing the real data from the $22.4 \mathrm{~km}$ baseline, whereas full ambiguity resolution should often only lead to meter level errors.

According to the ionospheric model, the ionospheric uncertainty for the CUT0-PERT baseline is given by $\sigma_{I}=12.7 \mathrm{~mm}$. No differential tropospheric delays are assumed to be present. The single epoch positioning results for the four ambiguity resolution schemes IB FAR, IB PAR, DT FAR F, and DT PAR F are presented in Figures 6.13, 6.14, 6.15, and 6.16, which can be read as follows. The east, north, and up errors are the difference between the computed position and precise reference coordinates. The position errors are color coded, where gray means that no ambiguity is resolved and blue means that all $n$ ambiguities are resolved. For the partial ambiguity resolution schemes, all shades in between are possible. The ratio of resolved ambiguities $|\mathcal{I}| / n$ is shown in the fourth row, and the resulting formal precision $\alpha$ in the fifth row. The feasible precision values are limited by the float and fully fixed solutions, which are drawn as blue lines.

Figure 6.13 shows the results of IB FAR. The bootstrapping failure rate of GPS only positioning never drops to below $0.1 \%$, which means that the number of fixed ambiguities is always zero 


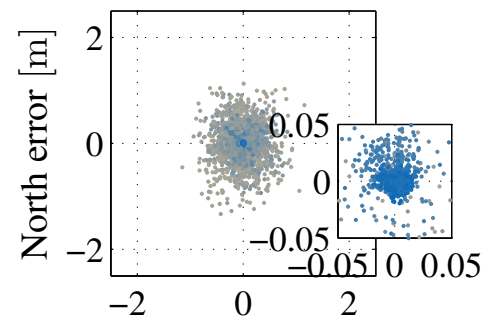

East error $[\mathrm{m}]$
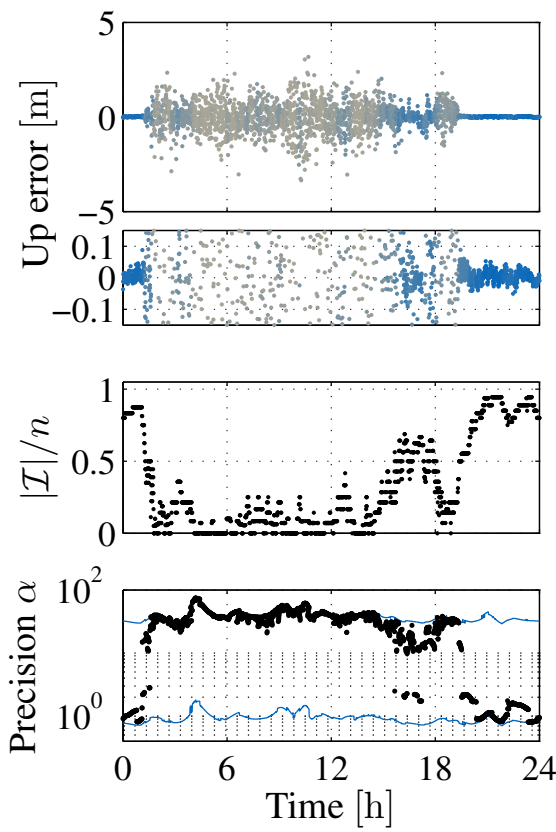

(a) GPS L1+L2

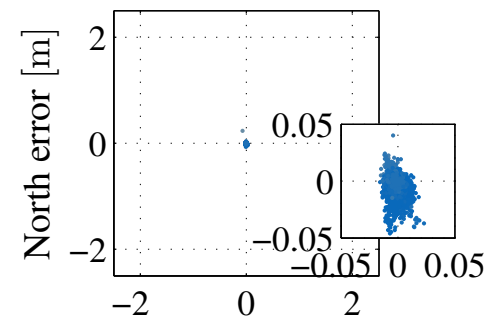

East error $[\mathrm{m}]$
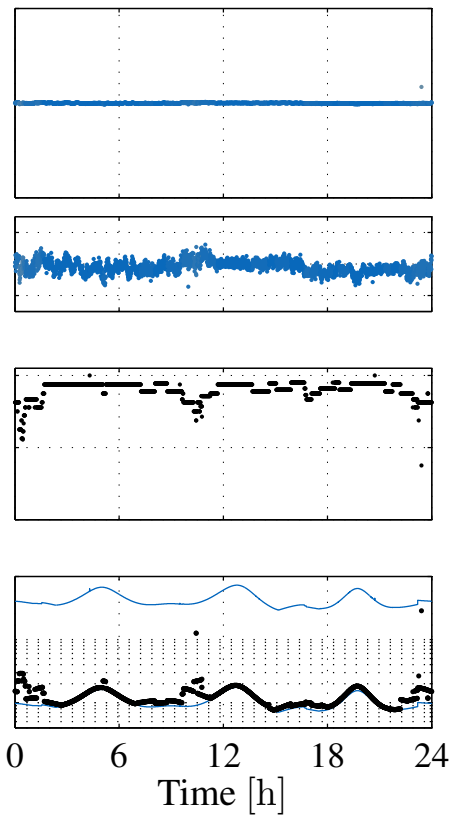

(b) BDS B1+B2

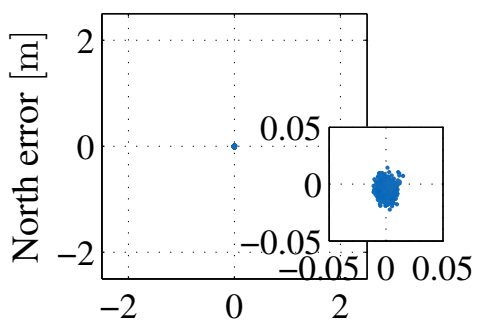

East error $[\mathrm{m}]$
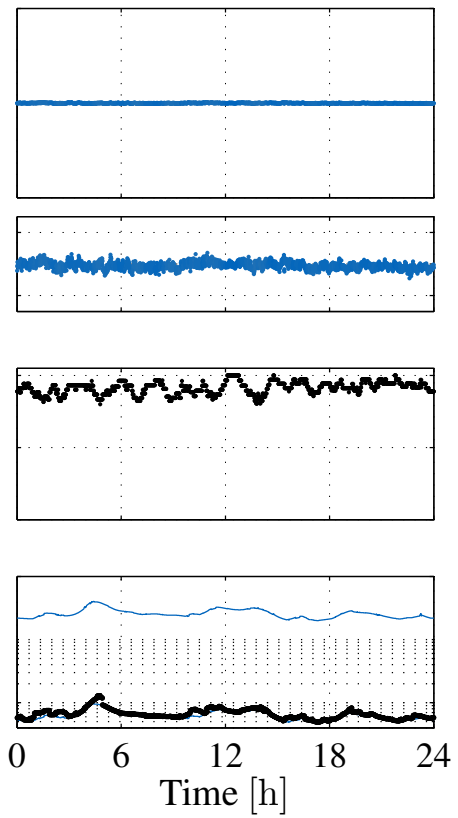

(c) GPS L1+L2 and BDS B1+B2

Figure 6.14: Dual frequency instantaneous RTK positioning results for CUT0-PERT with IB PAR and $P_{\mathrm{F}} \leq 0.1 \%$, see Caption of figure 6.13 .

and the formal precision $\alpha$ is always equal to the float precision. For BDS only, the full set of ambiguities can be resolved in two out of 2,880 time instances, and for combined GPS+BDS in $3.26 \%$ of all epochs, resulting in the blue position errors and small formal precision values.

The IB PAR results in Figure 6.14 are much more promising. For GPS only positioning, we can see how a high ratio of fixed ambiguities generally leads to a better formal precision $\alpha$, which coincides very well with the resulting position error, as can be seen in the up component. The benefit of partial ambiguity resolution is even more obvious if we consider the BDS and combined GPS+BDS results. The ratio of fixed ambiguities is always close to one, although the value one is almost never reached, except when IB PAR also reaches a ratio of one. Still, the formal precision $\alpha$ is always close to the best possible, fully fixed precision, which is confirmed by the resulting position errors.

As shown in Figure 6.15, with the data-driven DT FAR F scheme the fixed precision is reached in $62.05 \%$ of all epochs for GPS only, in $93.72 \%$ for BDS only, and in $75.76 \%$ for combined GPS+BDS. Although this is a clear improvement compared to the model-driven IB FAR, the availability of a precise position solution is still far from $100 \%$. The blue dots in the scatter plots of the east and north position errors also show that the precision of the fully fixed coordinates is higher 


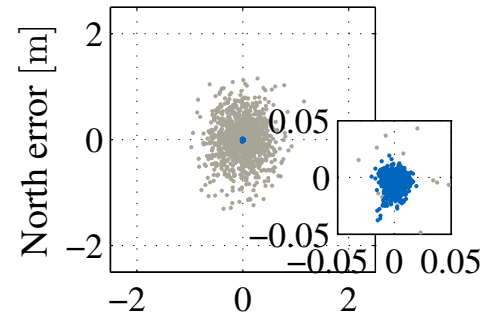

East error $[\mathrm{m}]$
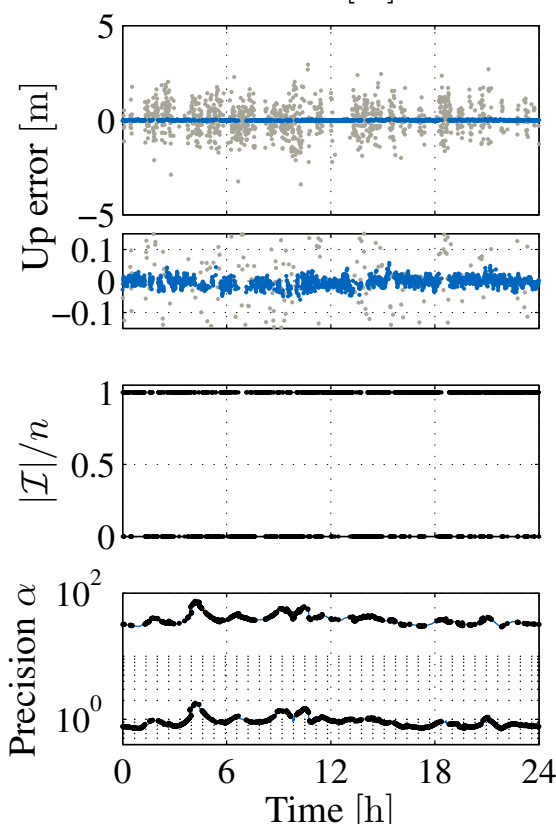

(a) GPS L1+L2

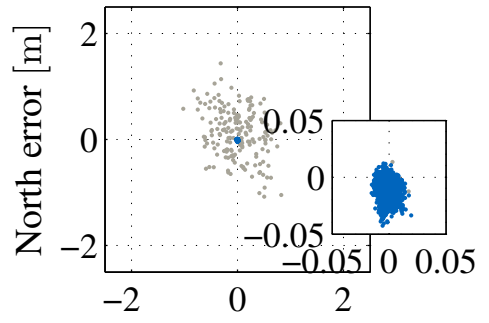

East error $[\mathrm{m}]$
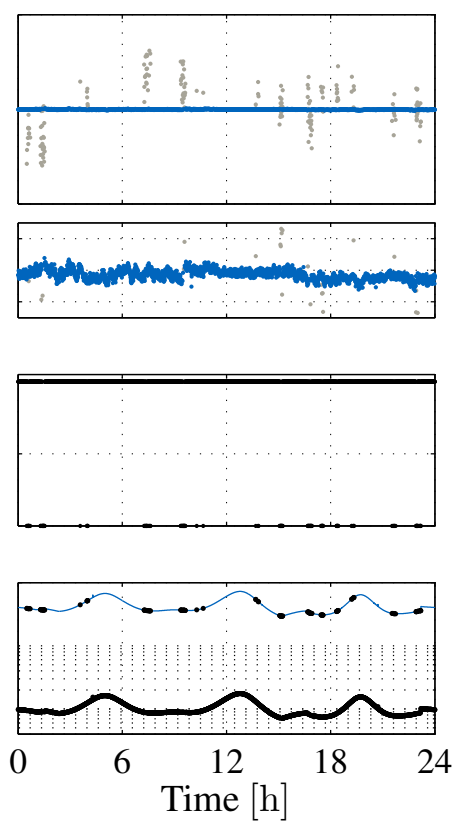

(b) BDS B1+B2

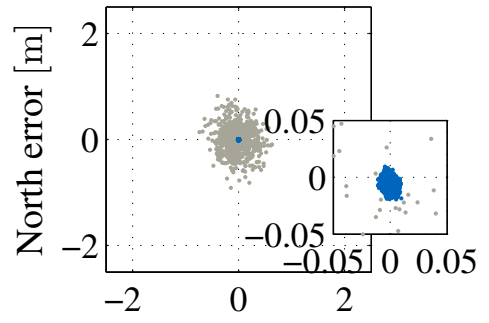

East error $[\mathrm{m}]$
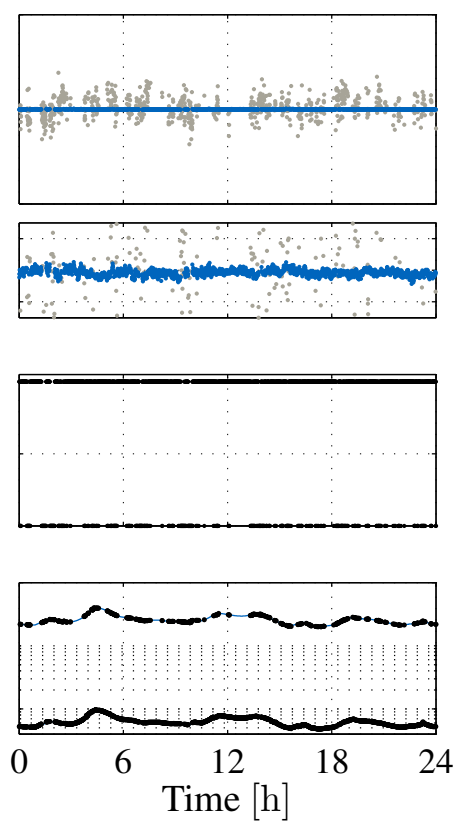

(c) GPS L1+L2 and BDS B1+B2

Figure 6.15: Dual frequency instantaneous RTK positioning results for CUT0-PERT with DT FAR F and $P_{\mathrm{F}} \leq 0.1 \%$, see Caption of figure 6.13 . 


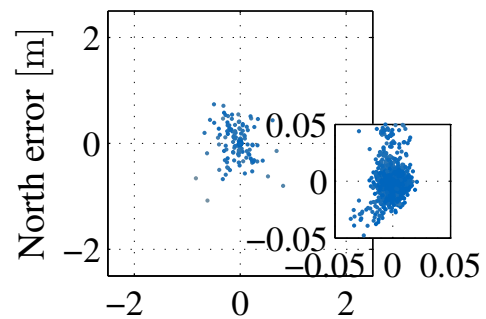

East error $[\mathrm{m}]$
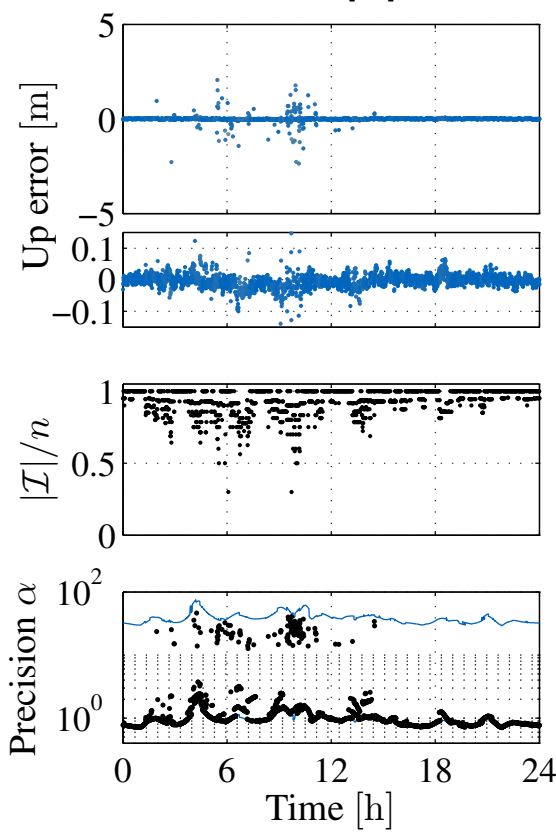

(a) GPS L1+L2

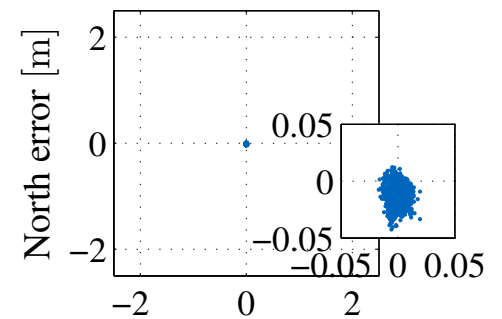

East error $[\mathrm{m}]$
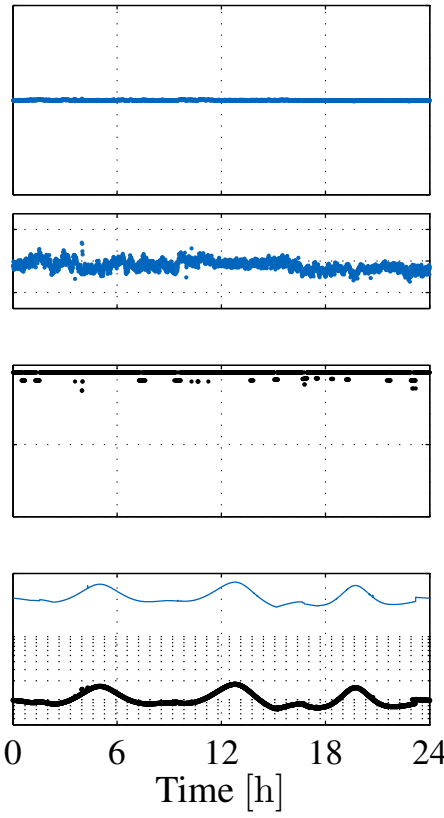

(b) BDS B1+B2

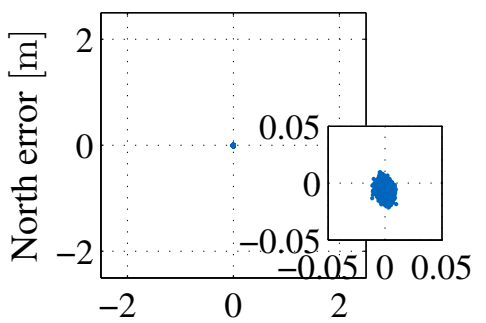

East error $[\mathrm{m}]$
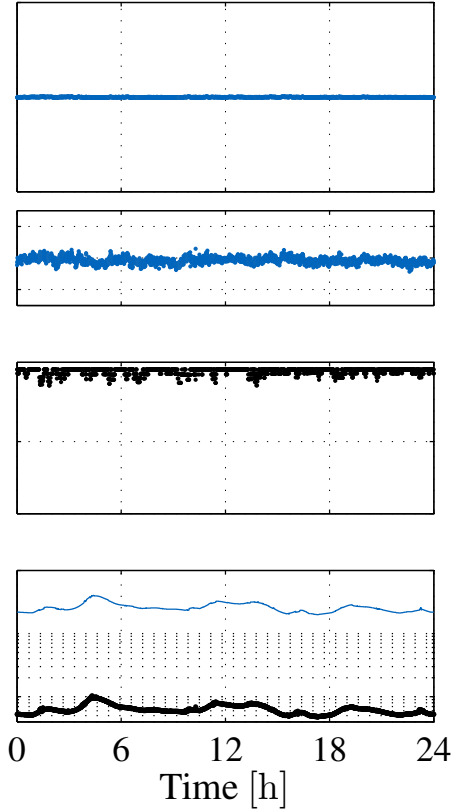

(c) GPS L1+L2 and BDS B1+B2

Figure 6.16: Dual frequency instantaneous RTK positioning results for CUT0-PERT with DT PAR F and $P_{\mathrm{F}} \leq 0.1 \%$, see Caption of figure 6.13 .

for the combined system than it is for the single systems. This is in accordance with the computed formal precision values.

The best results are obtained with DT PAR F as shown in Figure 6.16. The ratios of fixed ambiguities are further increased compared to IB PAR, resulting in better formal precision values and also smaller position errors. Resolving the full set of ambiguities is often not required in order to achieve close to optimal positioning precision. Compared to DT FAR F, the availability of precise centimeter level coordinate estimates is clearly larger for GPS only, and even reaches $100 \%$ for BDS only and combined GPS+BDS.

Figure 6.17 shows the cumulative rates of occurrence of the computed formal precision $\alpha$ for the same data. The gray dashed lines represent the maximum value of $\alpha$ that is obtained for the fully fixed solution during the whole day. That is, roughly speaking, everything left of the dashed line corresponds to a fully fixed precision. This figure summarizes what has been observed. With IB FAR (black), hardly ever anything better than the float precision is obtained. For DT FAR F (blue), we can see the step in each subfigure that corresponds to the rate of resolving the ambiguities, i.e., the rate of reaching the fully fixed precision. The two partial ambiguity resolution schemes in green and orange outperform their full ambiguity resolution counterparts, 


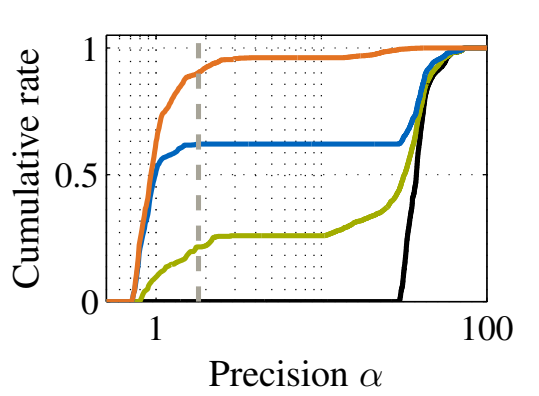

(a) GPS L1+L2

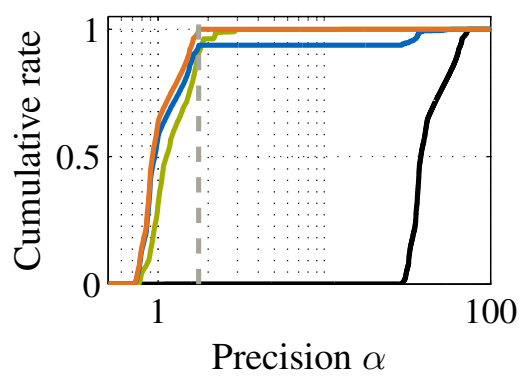

(b) BDS B1+B2

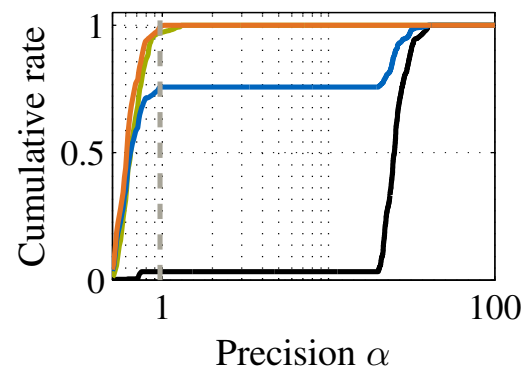

(c) GPS L1+L2 and BDS B1+B2

Figure 6.17: Cumulative rates of occurrence of formal precision $\alpha$ on CUT0-PER with $P_{\mathrm{F}} \leq 0.1 \%$; black: IB FAR, green: IB PAR, blue: DT FAR F, orange DT PAR F; the gray dashed lines indicate the maximum values of the fully fixed precision.

and always lead to precise coordinates for BDS only and combined GPS+BDS positioning. The results presented in this section are published in Brack (2017b).

\subsubsection{Long Baseline RTK Positioning on PERT-NNOR}

Like in the simulation analysis in Section 6.2.3, the double difference ionospheric slant delays and the between receiver differential tropospheric zenith delay are estimated for the long $88.5 \mathrm{~km}$ baseline PERT-NNOR, so that instantaneous centimeter level positioning is no longer possible. The measurements are a priori corrected for the hydrostatic tropospheric delays using the blind MOPS tropospheric model (MOPS 1999) and the Niell mapping function (Niell 1996). The float solutions are computed using a recursive least-squares implementation, in which the ambiguities are assumed time constant. The relative tropospheric wet zenith delay is modeled as a random walk with a process noise of $2 \frac{\mathrm{mm}}{\sqrt{\mathrm{h}}}$, and the user coordinates and ionospheric delays are assumed completely unlinked in time, meaning that the model is also valid in the possible presence of sudden ionospheric perturbations. Rising satellites are included immediately in the computation of the float solution. The ambiguity resolution methods are applied anew in each epoch, i.e., the ambiguities are not held fixed once an integer solution has been accepted. This allows for a better interpretation of the properties of different fixing methods other than the time to first fix.

Table 6.5: Average convergence times of IB FAR in terms of the required number of epochs; one epoch corresponds to $30 \mathrm{~s}$.

\begin{tabular}{lll}
\hline & $\alpha \leq 2$ & $\alpha \leq 10$ \\
\hline GPS L1+L2 & 58.7 & 52.3 \\
BDS B1+B2 & 45.1 & 44.3 \\
GPS L1+L2+ BDS B1+B2 & 26.2 & 21.0 \\
\hline
\end{tabular}

We start with a simulation based analysis for the given satellite to receiver geometry of the real data, in which the times required to reach centimeter level positioning results are predicted. The average convergence times, defined as the number of epochs that have to be collected with IB FAR in order to reach a formal precision of $\alpha \leq 2$ or $\alpha \leq 10$, are given in Table 6.5, where the estimation is started every $10 \mathrm{~min}$ throughout the day. Compared to the simulation results in Section 6.2.3, the values for $\alpha \leq 10$ are now only slightly smaller than the ones for $\alpha \leq 2$, which is caused by the different process model for the receiver coordinates. In Section 6.2.3 they are 

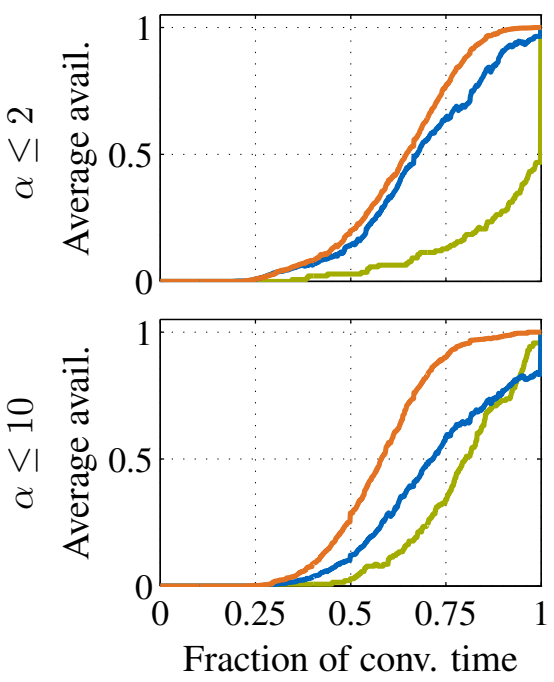

(a) GPS L1+L2
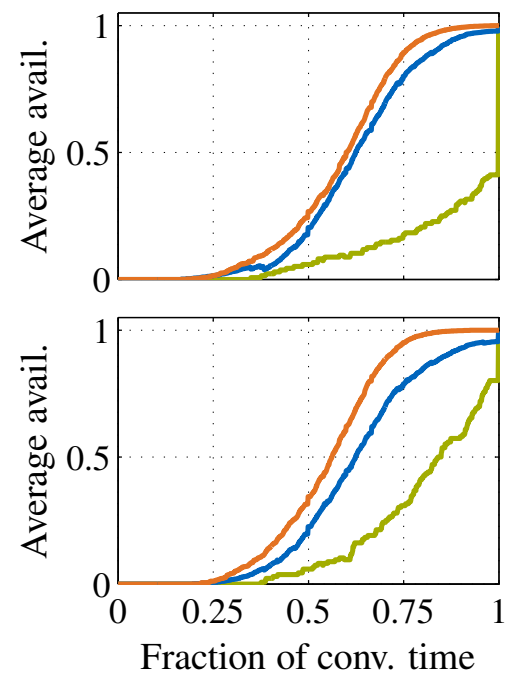

(b) BDS B1+B2
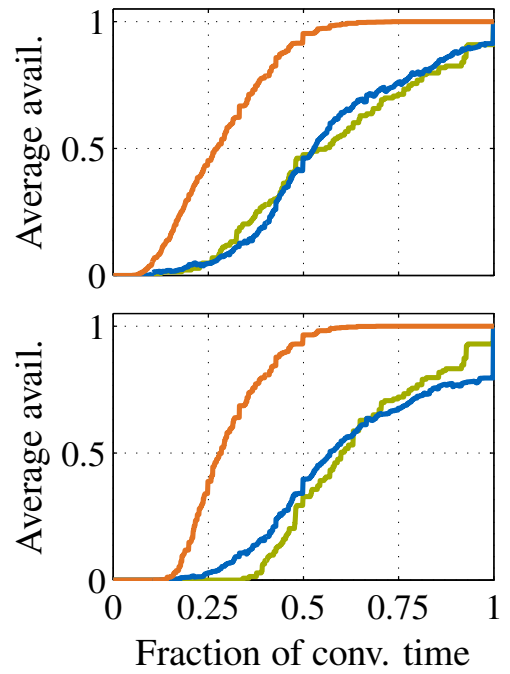

(c) GPS L1+L2 and BDS B1+B2

Figure 6.18: Dual frequency long baseline positioning with $P_{\mathrm{F}} \leq 0.1 \%$; green: IB PAR, blue: DT FAR F, orange: DT PAR F.

assumed time constant, so that the float solution is more likely to reach a level of $\alpha \leq 10$. Figure 6.18 shows by how much the convergence times can be expected to be reduced on average by using one of the more advanced ambiguity resolution schemes. For the combined system we can see that by using IB PAR or DT FAR F the time required to reach $\alpha \leq 2$ can be reduced by half with an average probability of $\sim 50 \%$, and by using DT PAR F even with an average probability of more than $95 \%$.

The positioning capabilities of the four ambiguity resolution schemes are now verified with real GNSS data for dual frequency combined GPS+BDS. The east, north, and up positioning errors as well as the ratio of fixed ambiguities $|\mathcal{I}| / n$ are shown for $45 \mathrm{~min}$ of data in Figure 6.19 for IB FAR and DT FAR F and in Figure 6.20 for the PAR counterparts. The positioning errors are the difference between the estimated position and precise reference coordinates. With IB FAR, 71 epochs of data are required until the integer bootstrapping failure rate drops to below $0.1 \%$, i.e., until the full set of integer ambiguities is resolved and the fixed solution can be accepted. With the data-driven DT FAR F, the ambiguities can already be resolved after 52 epochs. With IB PAR, the first ambiguities are resolved after 11 epochs, and after 14 epochs the estimates of the horizontal position components are already at centimeter level, although the full set of ambiguities is again only resolved after 71 epochs. An even faster solution is obtained with DT PAR F, for which the horizontal positioning error is at centimeter level after only 9 epochs. It is noted that the large numbers of epochs required by the full ambiguity resolution schemes are to some extend also caused by rising satellites after epoch 20 and 34. The partial ambiguity resolution schemes are almost not affected by this. Although the ratio of fixed ambiguities is slightly smaller immediately after the new satellite has been included, the positioning precision is not visibly reduced.

The positioning results for the whole day are shown in Figure 6.21 for reliable full ambiguity resolution and in Figure 6.22 for reliable partial ambiguity resolution. Again the position errors are color coded, where gray means that no ambiguity is resolved and blue means that all $n$ ambiguities are resolved. The ratio of resolved ambiguities is shown in the third row, and the computed formal precision $\alpha$ in the bottom row. The float and fully fixed solutions, which limit the feasible precision values, are drawn as blue lines. Since we are mostly interested in the time required to reach 


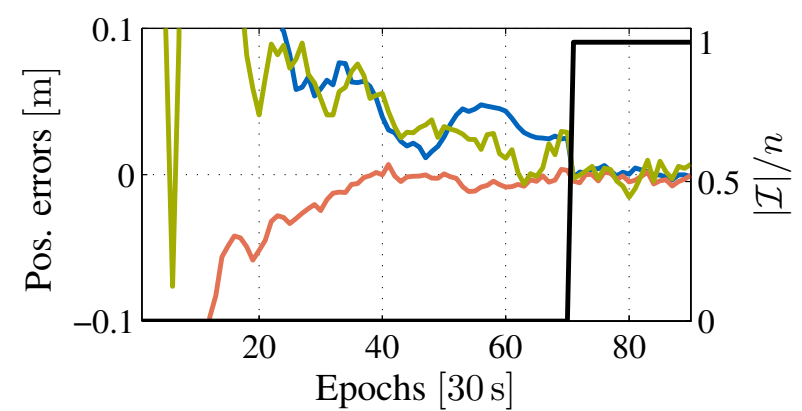

(a) IB FAR

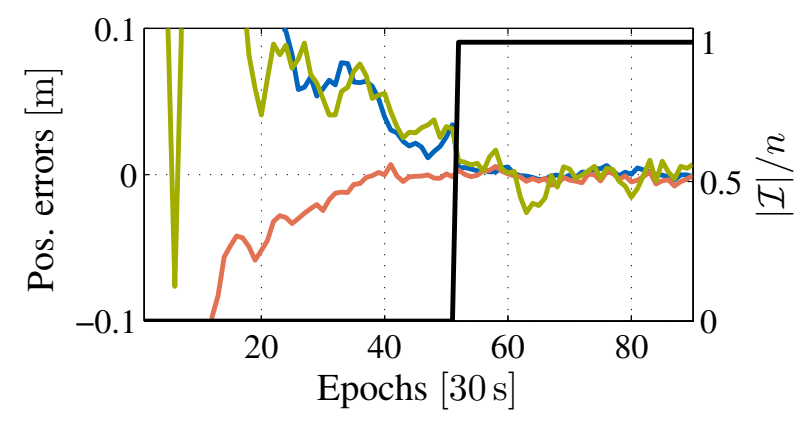

(b) DT FAR F

Figure 6.19: East (blue), north (orange), and up (green) positioning error with full ambiguity resolution for dual frequency combined GPS+BDS positioning on PERT-NNOR with $P_{\mathrm{F}} \leq 0.1 \%$; the ratio of fixed ambiguities is shown in black.

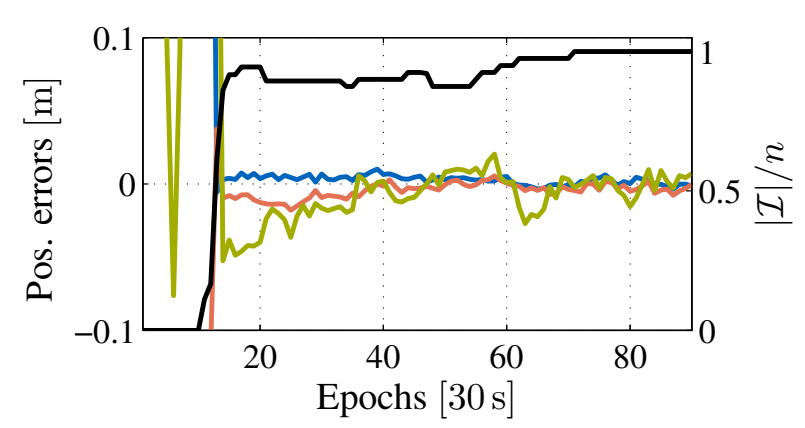

(a) IB PAR

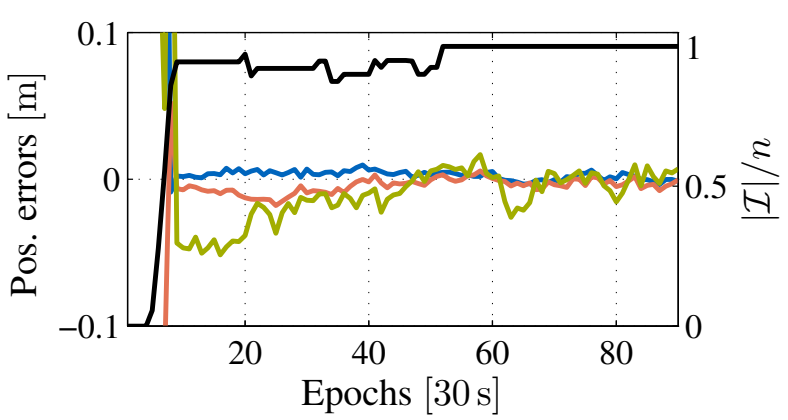

(b) DT PAR F

Figure 6.20: East (blue), north (orange), and up (green) positioning error with partial ambiguity resolution for dual frequency combined GPS+BDS positioning on PERT-NNOR with $P_{\mathrm{F}} \leq 0.1 \%$; the ratio of fixed ambiguities is shown in black. 

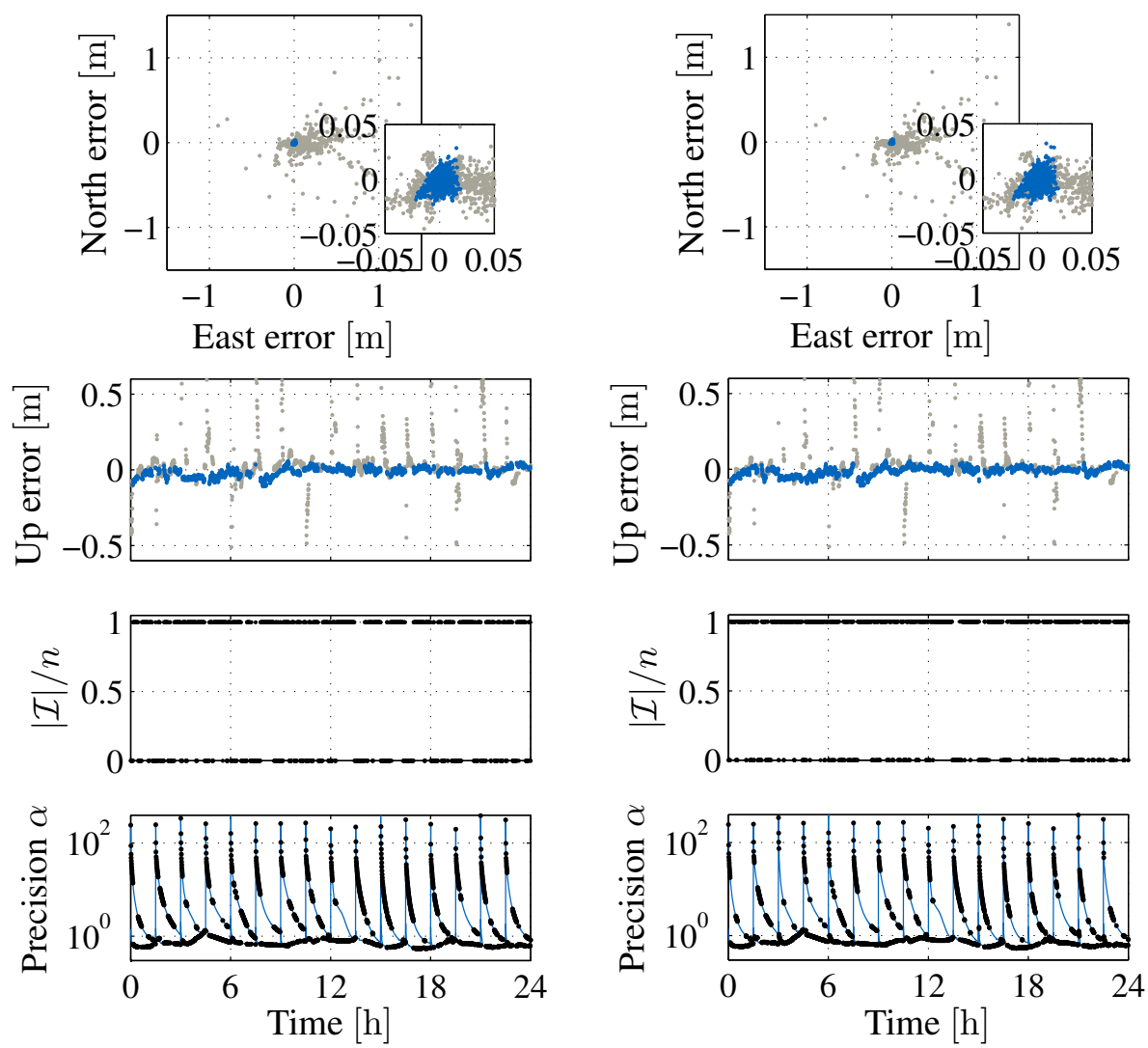

(a) IB FAR

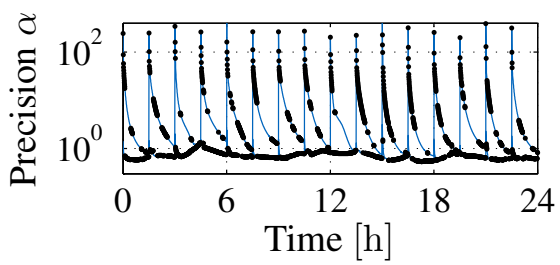

(b) DT FAR F

Figure 6.21: Long baseline dual frequency GPS+BDS positioning results for PERT-NNOR with full ambiguity resolution and $P_{\mathrm{F}} \leq 0.1 \%$. The color of the horizontal and vertical components of the positioning error indicates the ratio of fixed ambiguities as shown in the third row, where gray stands for a ratio of 0 and blue for a ratio of 1 . In the bottom row, the computed formal precision values $\alpha$ are shown in black. They are limited by the float (upper blue line) and fully fixed (lower blue line) precision. The estimator is reinitialized every $90 \mathrm{~min}$.

centimeter level precision, the estimator is completely reinitialized every $90 \mathrm{~min}$, as can most notably be seen at the formal float precision.

The ratio of fixed ambiguities for the full ambiguity resolution schemes in Figure 6.21 is either zero or one, corresponding to the gray and blue position errors, respectively. The horizontal error of the fully fixed solution is at centimeter level, whereas the gray float solution can have much larger errors, especially after reinitialization, as can be seen in the time series of the up error. The behavior after reinitialization agrees with the computed float precision values in the last row. Although the results of IB FAR and DT FAR F seem to be very similar, the fixed solution was accepted in 1,729 out of 2,880 epochs for IB FAR but in 2,011 epochs for DT FAR F. This difference is visible, e.g., in the up error after the last initialization. We further notice that the fixed solution is often lost when a satellite rises, as can be seen in the last row: The computed formal precision is identical to the float precision after initialization, before it drops to the fixed precision once the ambiguities are resolved. But then quite often the float precision is observed again for a couple of epochs at a later time instance. One could of course exclude rising satellites from the ambiguity fixing, which would be nothing else than a specific implementation of partial ambiguity resolution. 

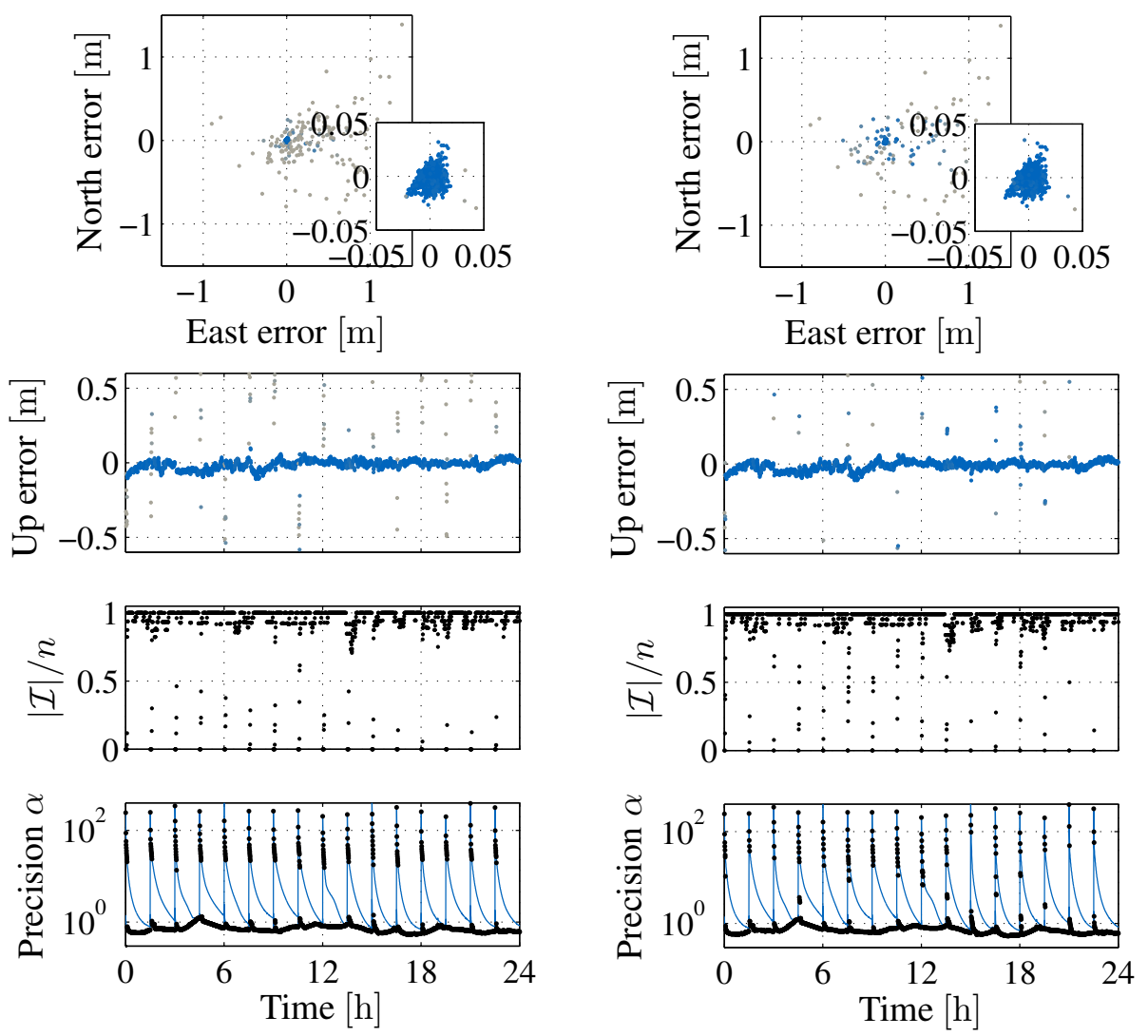

(a) IB PAR

(b) DT PAR F

Figure 6.22: Long baseline dual frequency GPS+BDS positioning results for PERT-NNOR with partial ambiguity resolution and $P_{\mathrm{F}} \leq 0.1 \%$, see caption of Figure 6.21 .

The ratio of fixed ambiguities for the partial ambiguity resolution strategies as shown in Figure 6.22 is mostly close to one, except for a few epochs after each reinitialization. Accordingly, the computed formal precision values in the last row are mostly very close to the fully fixed precision. This is verified by the position errors, where the number of epochs with large errors is significantly reduced compared to Figure 6.21. The best results are obtained for DT PAR F, for which the gray dots are almost completely gone and only a few epochs after each initialization remain with a position error that exceeds a few centimeters. Note that the blue dots with large positioning errors reflect the precision of partially fixed solutions and do not imply wrong ambiguity estimates. The average ratio of fixed ambiguities is $91.3 \%$ for IB PAR and $94.9 \%$ for DT PAR F (as compared to $60.0 \%$ and $69.8 \%$ for the FAR counterparts). We can also see that-unlike for full ambiguity resolution-rising satellites hardly affect the positioning performance and are thus not an issue with partial ambiguity resolution.

Summarizing, two main advantages of partial ambiguity resolution over full ambiguity resolution are identified in the context of long baseline positioning. The average number of epochs until the precision of the coordinate estimates first reaches centimeter level can be clearly reduced, and the problem of when or how to include the carrier-phase ambiguities corresponding to rising satellites in a multi-epoch positioning solution is automatically taken care of by the presented partial ambiguity resolution methods. The results presented in this section are published in Brack (2017a). 



\section{Integer Equivariant Estimation}

The discrete nature of the domain $\mathbb{Z}^{n}$ of the integer parameters $\boldsymbol{a}$ makes it possible to correctly estimate $\boldsymbol{a}$ with non-zero probability. In GNSS positioning applications, a correct estimate of $\boldsymbol{a}$ implies that one can fully exploit the low measurement noise of the carrier-phase observations. Mathematically this means that if an integer estimator is applied, i.e., if the full set of integer parameters is resolved without an additional acceptance test, and if the corresponding probability of correct integer estimates $P(\check{\boldsymbol{a}}=\boldsymbol{a})$ is sufficiently close to one, then the ambiguity fixed estimate $\check{\boldsymbol{b}}$ has a higher probability of being close to the true $\boldsymbol{b}$ than the float estimate $\hat{\boldsymbol{b}}$. As we could see in the previous chapter, there are many challenging positioning scenarios in which this success rate criterion does not hold. In order to avoid the large positioning errors that potentially result from incorrect ambiguity estimates, the strategy that has been used in this dissertation up to this point is to formulate reliable ambiguity resolution schemes with a user controlled maximum tolerable failure rate. Partial ambiguity resolution techniques were shown to be very helpful in terms of improving the positioning capabilities under such a failure rate constraint.

In this chapter a different option for dealing with weak positioning models is presented. The fundamental idea is to utilize the integer property of the carrier-phase ambiguities without fixing them to integers. Such estimators can be formulated within the class of integer equivariant estimators, which is defined in Teunissen (2003c) and presented in Section 7.1. The best integer equivariant estimator and two suboptimal realizations are shown in Section 7.2. Some fundamental properties of integer equivariant estimation in comparison with the float and (partially) ambiguity fixed solutions are demonstrated in Section 7.3 using a GNSS example.

\subsection{The Class of Integer Equivariant Estimators}

We recall from Section 2.4 that the considered problem is the estimation of the integer valued parameter vector $\boldsymbol{a} \in \mathbb{Z}^{n}$ or the joint estimation of $\boldsymbol{a}$ and the real valued parameter vector $\boldsymbol{b} \in \mathbb{R}^{p}$ in a linear observation model with additive zero mean Gaussian noise. The float solution $\hat{\boldsymbol{a}}$ and, if present, $\hat{b}$ are a sufficient statistic and can therefore be used as the basis for the definition of an integer equivariant estimator instead of the measurement vector $\boldsymbol{y}$. In Teunissen (2003c), an estimator $\hat{\theta}_{\mathrm{IE}}=F_{\theta}(\hat{\boldsymbol{a}}, \hat{\boldsymbol{b}})$ with $F_{\theta}: \mathbb{R}^{n+p} \mapsto \mathbb{R}$ is defined to be an integer equivariant estimator of an arbitrary linear combination $\theta=\boldsymbol{l}_{\boldsymbol{a}}^{\mathrm{T}} \boldsymbol{a}+\boldsymbol{l}_{\boldsymbol{b}}^{\mathrm{T}} \boldsymbol{b}$ of the parameters, if

$$
\begin{aligned}
F_{\theta}(\boldsymbol{x}+\boldsymbol{z}, \boldsymbol{\beta}) & =F_{\theta}(\boldsymbol{x}, \boldsymbol{\beta})+\boldsymbol{l}_{\boldsymbol{a}}^{\mathrm{T}} \boldsymbol{z}, & & \forall \boldsymbol{x} \in \mathbb{R}^{n}, \boldsymbol{\beta} \in \mathbb{R}^{p}, \boldsymbol{z} \in \mathbb{Z}^{n} \\
F_{\theta}(\boldsymbol{x}, \boldsymbol{\beta}+\boldsymbol{w}) & =F_{\theta}(\boldsymbol{x}, \boldsymbol{\beta})+\boldsymbol{l}_{\boldsymbol{b}}^{\mathrm{T}} \boldsymbol{w}, & & \forall \boldsymbol{x} \in \mathbb{R}^{n}, \boldsymbol{\beta} \in \mathbb{R}^{p}, \boldsymbol{w} \in \mathbb{R}^{p} .
\end{aligned}
$$

If the vector of real valued parameters $\boldsymbol{b}$ is absent in the system model so that there are only integer valued parameters, we have $\theta=\boldsymbol{l}_{\boldsymbol{a}}^{\mathrm{T}} \boldsymbol{a}$, the second slot of $F_{\theta}(\cdot)$ does not exist, and the second line of (7.1) does no longer apply. This case is not considered separately in the following.

In order to better understand (7.1), two integer equivariant estimators with specific choices of $\boldsymbol{l}_{\boldsymbol{a}}$ and $\boldsymbol{l}_{\boldsymbol{b}}$ are now investigated. According to (7.1), the estimator $\hat{\boldsymbol{a}}_{\mathrm{IE}}=F_{\boldsymbol{a}}(\hat{\boldsymbol{a}}, \hat{\boldsymbol{b}})$ with $F_{\boldsymbol{a}}: \mathbb{R}^{n+p} \mapsto$ $\mathbb{R}^{n}$ is an integer equivariant estimator of the integer parameters $\boldsymbol{a}$, if

$$
F_{\boldsymbol{a}}(\boldsymbol{x}+\boldsymbol{z}, \boldsymbol{\beta}+\boldsymbol{w})=F_{\boldsymbol{a}}(\boldsymbol{x}, \boldsymbol{\beta})+\boldsymbol{z}, \quad \forall \boldsymbol{x} \in \mathbb{R}^{n}, \boldsymbol{\beta} \in \mathbb{R}^{p}, \boldsymbol{z} \in \mathbb{Z}^{n}, \boldsymbol{w} \in \mathbb{R}^{p} .
$$


That is, if the float solution $\hat{\boldsymbol{a}}$ of $\boldsymbol{a}$ is shifted by an arbitrary integer, then the integer equivariant estimate $\hat{\boldsymbol{a}}_{\mathrm{IE}}$ is shifted by the same amount. It is noted that no integer constraints are in place for the estimate $\hat{\boldsymbol{a}}_{\mathrm{IE}}$. On the other hand, an arbitrary shift of $\hat{\boldsymbol{b}}$ must not affect the estimate of the integer parameters $\boldsymbol{a}$. Similarly, the estimator $\hat{\boldsymbol{b}}_{\mathrm{IE}}=F_{\boldsymbol{b}}(\hat{\boldsymbol{a}}, \hat{\boldsymbol{b}})$ with $F_{\boldsymbol{b}}: \mathbb{R}^{n+p} \mapsto \mathbb{R}^{p}$ is an integer equivariant estimator of the real valued parameters $b$, if

$$
F_{\boldsymbol{b}}(\boldsymbol{x}+\boldsymbol{z}, \boldsymbol{\beta}+\boldsymbol{w})=F_{\boldsymbol{b}}(\boldsymbol{x}, \boldsymbol{\beta})+\boldsymbol{w}, \quad \forall \boldsymbol{x} \in \mathbb{R}^{n}, \boldsymbol{\beta} \in \mathbb{R}^{p}, \boldsymbol{z} \in \mathbb{Z}^{n}, \boldsymbol{w} \in \mathbb{R}^{p}
$$

The estimate $\hat{\boldsymbol{b}}_{\mathrm{IE}}$ is thus invariant to an arbitrary integer shift of $\hat{\boldsymbol{a}}$. An arbitrary shift of $\hat{\boldsymbol{b}}$ must be directly reflected in $\hat{b}_{\mathrm{IE}}$. For GNSS applications, these properties imply that if an arbitrary number of complete cycles is added to the carrier-phase observations, then the estimates of the carrierphase ambiguities is shifted by the same integer amount, whereas the estimates of the real valued parameters are not affected by this.

It can easily be seen that the linear unbiased estimator of $\theta$ given by

$$
\hat{\theta}_{\mathrm{LU}}=\boldsymbol{l}_{\boldsymbol{a}}^{\mathrm{T}} \hat{\boldsymbol{a}}+\boldsymbol{l}_{\boldsymbol{b}}^{\mathrm{T}} \hat{\boldsymbol{b}}
$$

fulfills the criteria (7.1) and is therefore an integer equivariant estimator. The float estimate $\hat{b}$ itself is therefore an integer equivariant estimate of $\boldsymbol{b}$. When deriving the best integer equivariant estimator in Section 7.2.1 according to some optimality criterion, it is thus guaranteed that the estimates are indeed at least as good as $\hat{b}$. We can now check, whether the fixed estimates $\check{\boldsymbol{b}}=\hat{\boldsymbol{b}}-\boldsymbol{Q}_{\hat{\boldsymbol{b}}_{\hat{a}_{\mathcal{I}}}} \boldsymbol{Q}_{\hat{\boldsymbol{a}}_{\mathcal{I}}}^{-1}\left(\hat{\boldsymbol{a}}_{\mathcal{I}}-\check{\boldsymbol{a}}\right)$ as defined in (2.84) or an arbitrary linear combination $\check{\theta}^{\prime}=\boldsymbol{l}_{\boldsymbol{b}}^{\mathrm{T}} \check{\boldsymbol{b}}$ thereof are also integer equivariant estimates of $\boldsymbol{b}$ or $\theta^{\prime}=\boldsymbol{l}_{\boldsymbol{b}}^{\mathrm{T}} \boldsymbol{b}$, with $\check{\boldsymbol{a}}$ and $\mathcal{I}$ from the class of (partial) integer estimators or (generalized) integer aperture estimators. The reason why no combinations of the parameters $\boldsymbol{a}$ are considered, i.e., why $\boldsymbol{l}_{\boldsymbol{a}}$ is taken as $\boldsymbol{l}_{\boldsymbol{a}}=\mathbf{0}$ in $\theta^{\prime}$, is that the above mentioned estimators were formulated such that $\check{\boldsymbol{a}}$ is only an estimate for $\boldsymbol{a}_{\mathcal{I}}$. An estimate for the whole parameter vector $\boldsymbol{a}$ is generally not defined. For GNSS positioning applications this is not a problem since one is in any case only interested in the estimates of $\boldsymbol{b}$. The classes of (partial) integer estimators and (generalized) integer aperture estimators as defined in Sections 3.1, 3.2, 4.1, and 5.1 are such that the following property holds. If the float solution $\hat{\boldsymbol{a}}$ is shifted by an arbitrary integer $z \in \mathbb{Z}^{n}$, the resulting subset $\mathcal{I}$ of integer parameters to be resolved remains unchanged, and the integer estimate $\check{\boldsymbol{a}}$ of $\boldsymbol{a}_{\mathcal{I}}$ is shifted by $\boldsymbol{z}_{\mathcal{I}}$. This shows that the first property in (7.1) is fulfilled by the estimator $\check{\theta}^{\prime}=\boldsymbol{l}_{\boldsymbol{b}}^{\mathrm{T}} \check{\boldsymbol{b}}$, namely that an arbitrary integer shift of $\hat{\boldsymbol{a}}$ does not affect $\check{\boldsymbol{b}}$. The second property in (7.1) directly follows from the definition of $\breve{b}$ and the fact that $\check{a}$ does not depend on $\hat{b}$. The fixed solution $\check{\boldsymbol{b}}$ computed with $\check{\boldsymbol{a}}$ from any (partial) integer estimator or (generalized) integer aperture estimator is therefore also an integer equivariant estimate of $\boldsymbol{b}$.

\subsection{Examples of Integer Equivariant Estimators}

Three specific realizations of integer equivariant estimators are presented in the following. The first one is referred to as the best integer equivariant estimator and leads to the smallest mean square error among the class of integer equivariant estimators. The other two examples are an element-wise and a sequential scalar approximation of this optimal estimator. 


\subsubsection{Best Integer Equivariant Estimation}

For the definition of the best integer equivariant estimator $\hat{\theta}_{\mathrm{BIE}}$ of $\theta=\boldsymbol{l}_{\boldsymbol{a}}^{\mathrm{T}} \boldsymbol{a}+\boldsymbol{l}_{\boldsymbol{b}}^{\mathrm{T}} \boldsymbol{b}$ the mean square error criterion is used in Teunissen (2003c), i.e.,

$$
\hat{\theta}_{\mathrm{BIE}}=\underset{F_{\theta}(\cdot)}{\operatorname{argmin}} \mathrm{E}\left[\left(F_{\theta}(\hat{\boldsymbol{a}}, \hat{\boldsymbol{b}})-\theta\right)^{2}\right],
$$

with $F_{\theta}(\cdot)$ a valid integer equivariant estimator that satisfies the properties (7.1). For the Gaussian distributed float estimates $\hat{\boldsymbol{a}}$ and $\hat{\boldsymbol{b}}$, the solution of this optimization problem is given by

$$
\hat{\theta}_{\mathrm{BIE}}=\boldsymbol{l}_{\boldsymbol{a}}^{\mathrm{T}} \hat{\boldsymbol{a}}_{\mathrm{BIE}}+\boldsymbol{l}_{\boldsymbol{b}}^{\mathrm{T}} \hat{\boldsymbol{b}}_{\mathrm{BIE}}
$$

with

$$
\begin{aligned}
\hat{\boldsymbol{a}}_{\mathrm{BIE}} & =\sum_{\boldsymbol{z} \in \mathbb{Z}^{n}} \boldsymbol{z} \cdot \frac{\exp \left(-\frac{1}{2}\|\hat{\boldsymbol{a}}-\boldsymbol{z}\|_{\boldsymbol{Q}_{\hat{a}}}^{2}\right)}{\sum_{\boldsymbol{u} \in \mathbb{Z}^{n}} \exp \left(-\frac{1}{2}\|\hat{\boldsymbol{a}}-\boldsymbol{u}\|_{\boldsymbol{Q}_{\hat{a}}}^{2}\right)}=\sum_{\boldsymbol{z} \in \mathbb{Z}^{n}} w_{\boldsymbol{z}}(\hat{\boldsymbol{a}}) \boldsymbol{z} \\
\hat{\boldsymbol{b}}_{\mathrm{BIE}} & =\hat{\boldsymbol{b}}-\boldsymbol{Q}_{\hat{\boldsymbol{b}} \hat{\boldsymbol{a}}} \boldsymbol{Q}_{\hat{\boldsymbol{a}}}^{-1}\left(\hat{\boldsymbol{a}}-\hat{\boldsymbol{a}}_{\mathrm{BIE}}\right) .
\end{aligned}
$$

In the Gaussian case, the computation of $\hat{\boldsymbol{b}}_{\mathrm{BIE}}$ therefore also follows the framework from Section 2.4, where the estimates of (some of) the integer parameters are fixed to integers. First, the float solutions $\hat{\boldsymbol{a}}$ and $\hat{\boldsymbol{b}}$ are computed. Then, an improved estimate of the integer parameters $\boldsymbol{a}$ is found based on $\hat{\boldsymbol{a}}$, which is used in the third step to correct $\hat{\boldsymbol{b}}$ by using the correlation between $\hat{\boldsymbol{a}}$ and $\hat{\boldsymbol{b}}$. The difference is that $\hat{\boldsymbol{a}}_{\mathrm{BIE}}$ is now the weighted sum of all $n$ dimensional integer vectors with non-binary weights $w_{\boldsymbol{z}}(\hat{\boldsymbol{a}}), \sum_{\boldsymbol{z} \in \mathbb{Z}^{n}} w_{\boldsymbol{z}}(\hat{\boldsymbol{a}})=1$, so that $\hat{\boldsymbol{a}}_{\mathrm{BIE}}$ is in general not integer valued. The pdf of $\hat{\boldsymbol{a}}_{\mathrm{BIE}}$ is shown in Figure 7.1 for a two dimensional example. It is star shaped and represented by three axes of high relative frequency, which correspond to the neighboring integers of $\boldsymbol{a}$, see also Verhagen and Teunissen (2005). A formulation of the best integer equivariant estimator for non-Gaussian measurement noise is given in Teunissen $(2003 \mathrm{c})$.

With $\mathrm{E}\left[\hat{\boldsymbol{a}}_{\mathrm{BIE}}\right]=\boldsymbol{a}$, and therefore $\mathrm{E}\left[\hat{\boldsymbol{b}}_{\mathrm{BIE}}\right]=\boldsymbol{b}$, it follows that the best integer equivariant estimator is unbiased, i.e., $\mathrm{E}\left[\hat{\theta}_{\mathrm{BIE}}\right]=\theta$. The mean square error is therefore identical to the variance and the best integer equivariant estimator is also the minimum variance unbiased estimator within the class of integer equivariant estimators. Since the float solution $\hat{b}$ and the fixed solution $\breve{b}$ are integer equivariant estimators of $\boldsymbol{b}$, it follows that the variance of any linear function $\boldsymbol{l}_{\boldsymbol{b}}^{\mathrm{T}} \hat{\boldsymbol{b}}_{\mathrm{BIE}}$ of $\hat{\boldsymbol{b}}_{\mathrm{BIE}}$ is at least as good as the variance of the corresponding linear functions of the float or (partially) fixed solutions, given that the fixed solution $\check{\boldsymbol{a}}$ that is used to compute $\check{\boldsymbol{b}}$ is unbiased. This statement is equivalent to

$$
\operatorname{Var}\left[\hat{\boldsymbol{b}}_{\mathrm{BIE}}\right] \preccurlyeq \operatorname{Var}[\hat{\boldsymbol{b}}] \quad \text { and } \operatorname{Var}\left[\hat{\boldsymbol{b}}_{\mathrm{BIE}}\right] \preccurlyeq \operatorname{Var}[\check{\boldsymbol{b}}]
$$

with $\check{\boldsymbol{a}}$ unbiased.

Since the computation of $\hat{\boldsymbol{a}}_{\mathrm{BIE}}$ in (7.7) includes a sum over infinitely many integer vectors $\boldsymbol{z}, \hat{\boldsymbol{a}}_{\mathrm{BIE}}$ can not be computed exactly but has to be approximated by replacing $\mathbb{Z}^{n}$ with a finite set. The same problem is discussed in Section 4.3.4 for computing the optimal partial integer estimator. The solution presented there follows the approach in Teunissen (2005d) and is to omit all integers whose contribution to the sum is too small. The domain of the sum is thus replaced by $\Theta_{\hat{\boldsymbol{a}}}^{\lambda}=\left\{\boldsymbol{z} \in \mathbb{Z}^{n} \mid\|\hat{\boldsymbol{a}}-\boldsymbol{z}\|_{\boldsymbol{Q}_{\hat{\boldsymbol{a}}}}^{2}<\lambda^{2}\right\}$, where $\lambda$ is chosen such that $P\left(\|\hat{\boldsymbol{a}}-\boldsymbol{a}\|_{\boldsymbol{Q}_{\hat{\boldsymbol{a}}}}^{2}<\lambda^{2}\right)=1-\alpha$ with a sufficiently small value of $\alpha$. The computation of the approximate $\hat{\boldsymbol{a}}_{\mathrm{BIE}}$ now includes a 


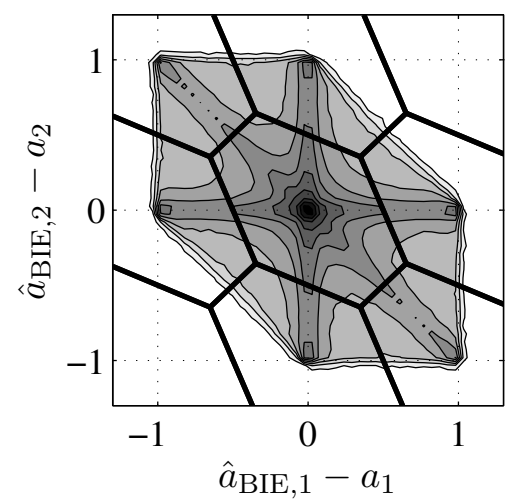

(a) Best integer equivariant estimator

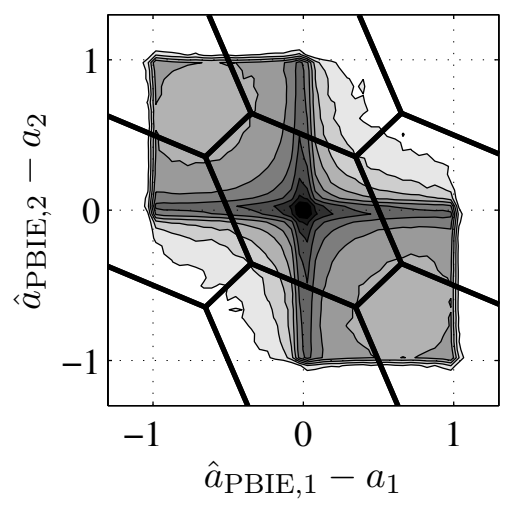

(b) Parallel scalar approximation

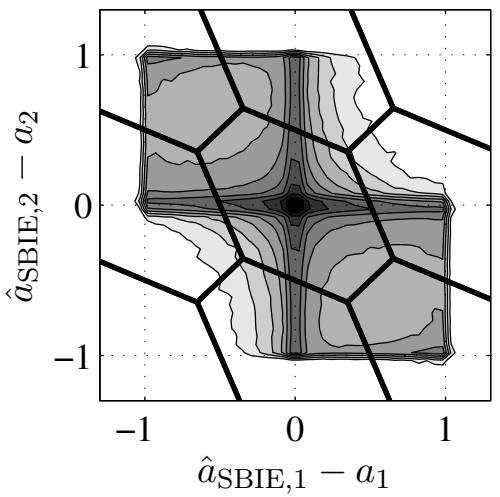

(c) Sequential scalar approximation

Figure 7.1: The relative frequencies of $\hat{\boldsymbol{a}}_{\mathrm{BIE}}, \hat{\boldsymbol{a}}_{\mathrm{PBIE}}$, and $\hat{\boldsymbol{a}}_{\mathrm{SBIE}}$ are illustrated for a two dimensional example, where dark areas represent high values; the colorscale is chosen logarithmic, i.e., the true distributions are more peaked than they appear to be; the integer least-squares pull-in regions indicate the shape of the Gaussian distribution of $\hat{\boldsymbol{a}}$.

search for all integers $z \in \Theta_{\hat{a}}^{\lambda}$. In order to reduce the involved computational complexity, this search should be carried out in a decorrelated space after an invertible transformation of the float solution $\hat{\boldsymbol{a}}$ with $\boldsymbol{Z}$. The best integer equivariant estimate $\hat{\boldsymbol{a}}_{\mathrm{BIE}}$ can be equivalently computed in any parameterization of $\hat{\boldsymbol{a}}$ that follows from a unimodular transformation. It is demonstrated in Teunissen (2003c) that $\hat{\boldsymbol{a}}_{\mathrm{BIE}}$ becomes identical to the float solution $\hat{\boldsymbol{a}}$ itself if $\hat{\boldsymbol{a}}$ is of very low precision, and that $\hat{\boldsymbol{a}}_{\mathrm{BIE}}$ converges to the integer least-squares solution $\check{\boldsymbol{a}}_{\mathrm{ILS}}$ if the pdf of $\hat{\boldsymbol{a}}$ gets very peaked compared to the integer grid.

The best integer equivariant estimates $\hat{\boldsymbol{a}}_{\mathrm{BIE}}$ and $\hat{\boldsymbol{b}}_{\mathrm{BIE}}$ are functionally identical to the Bayesian estimates of $\boldsymbol{a}$ and $\boldsymbol{b}$ when using the non-informative prior pdfs $p_{\boldsymbol{a}}(\boldsymbol{x}) \propto \sum_{\boldsymbol{z} \in \mathbb{Z}^{n}} \delta(\boldsymbol{z})$ and $p_{\boldsymbol{b}} \propto 1$ for the now assumed random $\boldsymbol{a}$ and $\boldsymbol{b}$ (Betti et al. 1993; Gundlich and Koch 2002), where $\delta(\cdot)$ is the Dirac function. A recursive GPS processing scheme that is also identical to the best integer equivariant estimator for the user position is proposed in the early papers Brown and Hwang (1983b) and Brown and Hwang (1983a). It is motivated by Magill's adaptive filtering concept (Magill 1965; Sims et al. 1969), which employs a bank of Kalman filters running in parallel on the same measurement data, but with a different modeling hypothesis. The final estimate is then computed as the weighted sum of the individual estimates. For GNSS applications, the hypotheses are different assumptions on the integer ambiguity vector $\boldsymbol{a}$, which is treated as known in each filter. The final estimate of $\boldsymbol{b}$ is the weighted sum of all estimates of $\boldsymbol{b}$ that are computed under different integer assumptions, which is identical to the best integer equivariant estimate $\hat{\boldsymbol{b}}_{\mathrm{BIE}}$ as defined in (7.7).

\subsubsection{Parallel Scalar Approximation}

Although an integer decorrelation and a proper tree search routine can dramatically reduce the complexity of finding all integer vectors within the search space $\Theta_{\hat{\boldsymbol{a}}}^{\lambda}$ required to compute $\hat{\boldsymbol{a}}_{\mathrm{BIE}}$ and $\hat{\boldsymbol{b}}_{\mathrm{BIE}}$, the complexity of the search might still be too high for certain applications, in particular when the number $n$ of integer parameters is large. Therefore, a suboptimal, but from a computational point of view less demanding approach is now introduced. It combines the idea of the optimal best integer equivariant estimator with a per element processing strategy, in which the correlations 


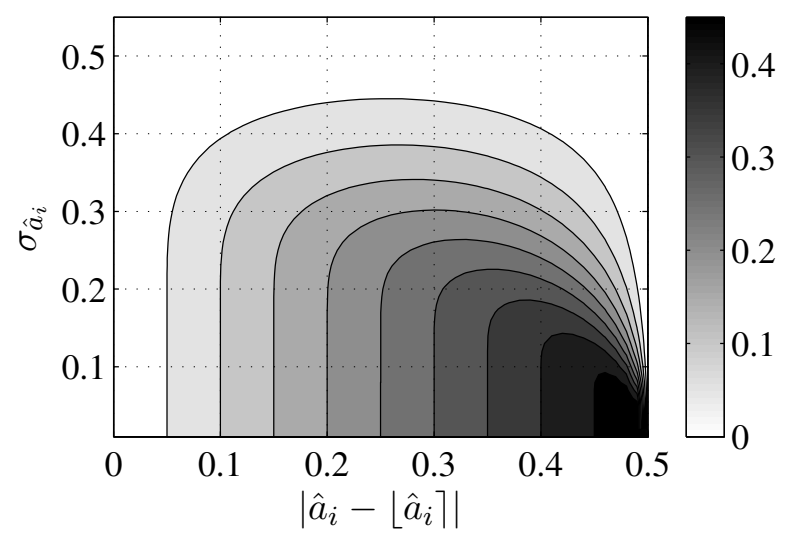

Figure 7.2: The different shades indicate the absolute value of the function $f\left(\hat{a}_{i}-\left\lfloor\hat{a}_{i}\right\rceil, \sigma_{\hat{a}_{i}}\right)$, where the sign of the function value is such that the float solution $\hat{a}_{i}$ gets pulled towards the closest integer $\left\lfloor\hat{a}_{i}\right\rceil$, i.e., it depends on the sign of $\hat{a}_{i}-\left\lfloor\hat{a}_{i}\right\rceil$.

between the elements are simply neglected. Instead of performing one $n$ dimensional search in the space of integers $\mathbb{Z}^{n}$, $n$ one dimensional searches are performed in parallel, i.e., the best integer equivariant estimate of each scalar component of $\hat{\boldsymbol{a}}$ is computed. The elements of this parallel scalar approximation $\hat{\boldsymbol{a}}_{\mathrm{PBIE}}$ of the best integer equivariant estimate $\hat{\boldsymbol{a}}_{\mathrm{BIE}}$ are given by

$$
\hat{a}_{\mathrm{PBIE}, i}=\sum_{z \in \mathbb{Z}} z \cdot \frac{\exp \left(-\frac{1}{2 \sigma_{\hat{a}_{i}}^{2}}\left|\hat{a}_{i}-z\right|^{2}\right)}{\sum_{u \in \mathbb{Z}} \exp \left(-\frac{1}{2 \sigma_{\hat{a}_{i}}^{2}}\left|\hat{a}_{i}-u\right|^{2}\right)}=\sum_{z \in \mathbb{Z}} w_{z}\left(\hat{a}_{i}\right) z, \quad i=1, \ldots, n .
$$

The corresponding estimate $\hat{\boldsymbol{b}}_{\mathrm{PBIE}}$ is computed as stated in (7.7), where $\hat{\boldsymbol{a}}_{\mathrm{BIE}}$ is replaced by $\hat{\boldsymbol{a}}_{\mathrm{PBIE}}$. It can easily be verified that the conditions (7.1) are fulfilled by the estimate $\hat{\theta}_{\mathrm{PBIE}}=\boldsymbol{l}_{\boldsymbol{a}}^{\mathrm{T}} \hat{\boldsymbol{a}}_{\mathrm{PBIE}}+$ $\boldsymbol{l}_{\boldsymbol{b}}^{\mathrm{T}} \hat{\boldsymbol{b}}_{\mathrm{PBIE}}$ of $\theta$, since an arbitrary integer shift of the float estimate $\hat{\boldsymbol{a}}$ results in the same integer shift of $\hat{\boldsymbol{a}}_{\text {PBIE }}$, but does not affect $\hat{\boldsymbol{b}}_{\text {PBIE. }}$. The second condition directly follows from the definition of $\hat{\boldsymbol{b}}_{\text {PBIE. }}$ The parallel scalar approximation is therefore a valid integer equivariant estimator. The pdf of $\hat{\boldsymbol{a}}_{\mathrm{PBIE}}$ is shown in Figure 7.1 for a two dimensional example. Due to the per element estimation, the diagonal axis of high relative frequency is no longer present, and only the two axes representing the two dimensional integer grid remain.

Essentially, the update step from the float estimate $\hat{a}_{i}$ to the scalar best integer equivariant solution $\hat{a}_{\mathrm{PBIE}, i}$ means that the float estimate $\hat{a}_{i}$ is pulled towards the closest integer $\left\lfloor\hat{a}_{i}\right\rceil$. The amount, by how much $\hat{a}_{i}$ is pulled, depends on only two parameters, namely the offset $\hat{a}_{i}-\left\lfloor\hat{a}_{i}\right\rceil$ and the standard deviation $\sigma_{\hat{a}_{i}}$. Let $f: \mathbb{R}^{2} \mapsto \mathbb{R}$ describe this relation as

$$
\hat{a}_{i}-\hat{a}_{\mathrm{PBIE}, i}=f\left(\hat{a}_{i}-\left\lfloor\hat{a}_{i}\right\rceil, \sigma_{\hat{a}_{i}}\right) .
$$

A graphical representation of $f(\cdot)$ is given in Figure 7.2. For very small values of $\sigma_{\hat{a}_{i}}$, the function $f(\cdot)$ becomes equal to the offset $\hat{a}_{i}-\left\lfloor\hat{a}_{i}\right\rceil$, so that $\hat{a}_{\mathrm{PBIE}, i}$ is essentially fixed to the closest integer $\left\lfloor\hat{a}_{i}\right\rceil$. The higher the uncertainty $\sigma_{\hat{a}_{i}}$ of the float estimate gets, the smaller the absolute value of $f(\cdot)$ gets compared to the absolute offset $\left|\hat{a}_{i}-\left\lfloor\hat{a}_{i}\right\rceil\right|$ itself. For values of $\sigma_{\hat{a}_{i}}$ larger than one half of a cycle, $\hat{a}_{\mathrm{PBIE}, i}$ only marginally differs from $\hat{a}_{i}$.

The limiting cases of very low and very high precision of $\hat{a}_{i}$ are now analyzed in more detail. If $\hat{\boldsymbol{a}}$ is of very low precision, the integer grid is very dense compared to the pdf of the float solution $\hat{\boldsymbol{a}}$. In the one dimensional case this means that there always exist pairs of integers around $\hat{a}_{i}$ with approximately the same distance and therefore the same weight $w_{z}\left(\hat{a}_{i}\right)$, see Figure 7.3. The weighted sum in (7.9) thus averages to the float solution $\hat{a}_{i}$ itself. Formally, the equality of $\hat{a}_{i}$ and 


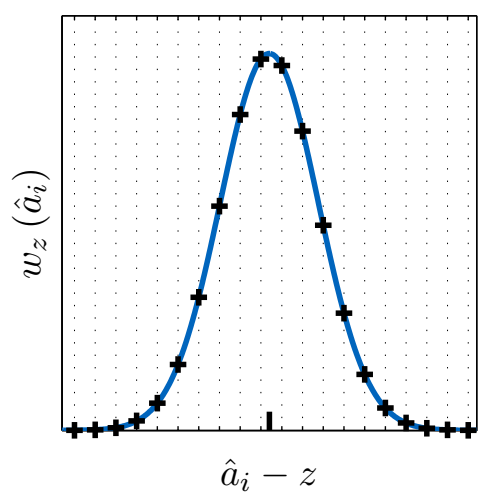

(a) Low precision of $\hat{a}_{i}$

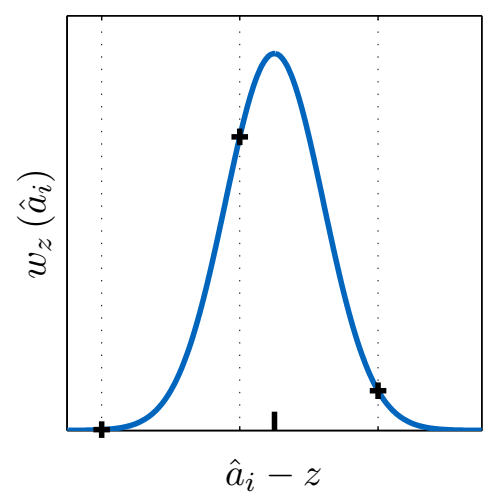

(b) High precision of $\hat{a}_{i}$

Figure 7.3: The weights $w_{z}\left(\hat{a}_{i}\right)$ of the integers-represented by dotted vertical lines-are marked in black. They are proportional to the value of the Gaussian curve centered around the float solution $\hat{a}_{i}$.

$\hat{a}_{\mathrm{PBIE}, i}$ is given for $\sigma_{\hat{a}_{i}} \rightarrow \infty$, which means that the summation in (7.9) is replaced by integration and we have

$$
\hat{a}_{\mathrm{PBIE}, i}=\int_{\mathbb{R}} z \cdot w_{z}\left(\hat{a}_{i}\right) \mathrm{d} z=\hat{a}_{i} .
$$

The second equality follows from $\int_{\mathbb{R}} w_{z}\left(\hat{a}_{i}\right) \mathrm{d} z=1$ and the fact that $w_{z}\left(\hat{a}_{i}\right)$ is symmetric with respect to $\hat{a}_{i}$. That is, like the best integer equivariant estimate $\hat{\boldsymbol{a}}_{\mathrm{BIE}}, \hat{\boldsymbol{a}}_{\mathrm{PBIE}}$ becomes identical to the float solution $\hat{a}$ if that is of very low precision.

Analogously, if $\hat{\boldsymbol{a}}$ is of very high precision, the pdf of $\hat{\boldsymbol{a}}$ is very peaked compared to the integer grid. This implies that the weight of one integer in (7.9) is considerably larger than the weight of all other integers, which is illustrated in Figure 7.3. With the weights approaching binary values, $\hat{a}_{\mathrm{PBIE}, i}$ converges to the closest integer $\left\lfloor\hat{a}_{i}\right\rceil$. This can be formally shown as follows. The weights in (7.9) can also be written as

$$
w_{z}\left(\hat{a}_{i}\right)=\frac{1}{1+\sum_{u \in \mathbb{Z} \backslash z} \exp \left(-\frac{1}{2 \sigma_{\hat{a}_{i}}^{2}}\left(\left|\hat{a}_{i}-u\right|^{2}-\left|\hat{a}_{i}-z\right|^{2}\right)\right)} .
$$

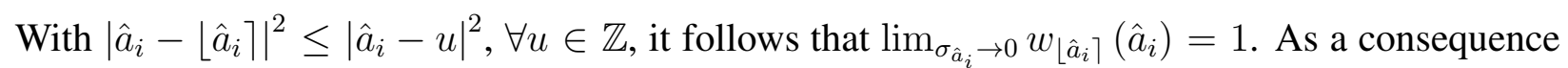
of $\sum_{z \in \mathbb{Z}} w_{z}\left(\hat{a}_{i}\right)=1$, with $w_{z}\left(\hat{a}_{i}\right)$ non-negative $\forall z \in \mathbb{Z}$, we also have that $\lim _{\sigma_{\hat{a}_{i} \rightarrow 0}} w_{z}\left(\hat{a}_{i}\right)=0$, $\forall z \in \mathbb{Z} \backslash\left\lfloor\hat{a}_{i}\right\rceil$, which shows that $\lim _{\sigma_{\hat{a}_{i} \rightarrow 0}} \hat{a}_{\mathrm{PBIE}, i}=\left\lfloor\hat{a}_{i}\right\rceil$. Accordingly, $\hat{\boldsymbol{a}}_{\mathrm{PBIE}}$ converges to the solution of element-wise rounding $\check{\boldsymbol{a}}_{\mathrm{IR}}$ if $\hat{\boldsymbol{a}}$ is of very high precision. This shows an interesting analogy with $\hat{\boldsymbol{a}}_{\mathrm{BIE}}$, which converges to $\check{\boldsymbol{a}}_{\mathrm{ILS}}$ if the precision of the float solution is very high. The estimate $\hat{\boldsymbol{a}}_{\mathrm{PBIE}}$ follows from the optimal $\hat{\boldsymbol{a}}_{\mathrm{BIE}}$ by neglecting the correlations between the elements of the float solution and by applying the optimal estimator to each element separately. This, however, is exactly how element-wise rounding $\check{\boldsymbol{a}}_{\mathrm{IR}}$ can be derived from $\check{\boldsymbol{a}}_{\mathrm{ILS}}$, which is optimal within the class of integer estimators.

It seems to be intuitively clear that the best integer equivariant estimate $\hat{\boldsymbol{a}}_{\mathrm{BIE}}$ of $\boldsymbol{a}$ can be written separately for each component, if no correlations between the elements of $\hat{\boldsymbol{a}}$ are present, which is now demonstrated. If $\boldsymbol{Q}_{\hat{\boldsymbol{a}}}$ is a diagonal matrix, then $\exp \left(-\frac{1}{2}\|\hat{\boldsymbol{a}}-\boldsymbol{z}\|_{\boldsymbol{Q}_{\hat{a}}}^{2}\right)=$ $\prod_{j=1}^{n} \exp \left(\left|\hat{a}_{j}-z_{j}\right|^{2} / \sigma_{\hat{a}_{j}}^{2}\right)=\prod_{j=1}^{n} g_{j}\left(z_{j}\right)$, where the factors $g_{j}\left(z_{j}\right)$ only depend on the $j$ th element of $\hat{\boldsymbol{a}}$. The $i$ th element of the best integer equivariant estimate $\hat{\boldsymbol{a}}_{\mathrm{BIE}}$ from (7.7) can now also 
be written as

$$
\hat{a}_{\mathrm{BIE}, i}=\frac{\sum_{\boldsymbol{z} \in \mathbb{Z}^{n}} z_{i} \prod_{j=1}^{n} g_{j}\left(z_{j}\right)}{\sum_{\boldsymbol{u} \in \mathbb{Z}^{n}} \prod_{j=1}^{n} g_{j}\left(u_{j}\right)} .
$$

The sum in the numerator of (7.13) can be decomposed into

$$
\begin{aligned}
\sum_{\boldsymbol{z} \in \mathbb{Z}^{n}} z_{i} \prod_{j=1}^{n} g_{j}\left(z_{j}\right) & =\sum_{v \in \mathbb{Z}} \sum_{\boldsymbol{z} \in \mathbb{Z}^{n} \mid z_{i}=v} v g_{i}(v) \prod_{\substack{j=1 \\
j \neq i}}^{n} g_{j}\left(z_{j}\right) \\
& =\left(\sum_{v \in \mathbb{Z}} v g_{i}(v)\right) \cdot\left(\sum_{\substack{\boldsymbol{z} \in \mathbb{Z}^{n} \mid z_{i}=0 \\
j=1 \\
j \neq i}}^{n} g_{j}\left(z_{j}\right)\right) .
\end{aligned}
$$

Note that in the second line the condition $z_{i}=v$ is replaced by $z_{i}=0$, which is allowed since the value of $z_{i}$ has no impact on the terms of the sum. This makes it possible to write the numerator of (7.13) as a product of two factors. Applying the same factorization to the denominator with $u$ instead of $z$ finally gives

$$
\hat{a}_{\mathrm{BIE}, i}=\sum_{z \in \mathbb{Z}} z \cdot \frac{g_{i}(z)}{\sum_{u \in \mathbb{Z}} g_{i}(u)} .
$$

This corresponds to a separate estimation of each element with a scalar version of the best integer equivariant estimator, which is identical to the parallel scalar implementation $\hat{\boldsymbol{a}}_{\mathrm{PBIE}}$, meaning that $\hat{\boldsymbol{a}}_{\text {PBIE }}$ is mean square error optimal if $\boldsymbol{Q}_{\hat{a}}$ is diagonal.

The resulting $\hat{\boldsymbol{a}}_{\mathrm{PBIE}}$ is not equivalent if one chooses to apply a prior unimodular integer transformation $\boldsymbol{Z}$ to the float solution $\hat{\boldsymbol{a}}$, thus also leading to a different $\hat{\boldsymbol{b}}_{\mathrm{PBIE}}$. Since $\hat{\boldsymbol{a}}_{\mathrm{PBIE}}$ and $\hat{\boldsymbol{b}}_{\mathrm{PBIE}}$ are optimal if the entries of $\hat{\boldsymbol{a}}$ are completely uncorrelated, it is recommended to compute $\hat{\boldsymbol{a}}_{\mathrm{PBIE}}$ in a decorrelated space, see Section 3.1.5. This transformation must be inverted or, equivalently, properly considered when computing $\hat{\boldsymbol{b}}_{\text {PBIE }}$ from $\hat{\boldsymbol{b}}$ according to (7.7).

For the implementation of the parallel scalar best integer equivariant approximation, the infinite sum in (7.9) required for the computation of each element is replaced by the sum over the finite set $\Theta_{\hat{a}_{i}}^{\lambda}$. In contrast to the multivariate case, the search for all integers within the search space $\Theta_{\hat{a}_{i}}^{\lambda}$ is a trivial task in a one dimensional space, as $\Theta_{\hat{a}_{i}}^{\lambda}$ is given by

$$
\Theta_{\hat{a}_{i}}^{\lambda}=\left\{z \in \mathbb{Z} \mid z \in\left[\hat{a}_{i}-\lambda \sigma_{\hat{a}_{i}}, \hat{a}_{i}+\lambda \sigma_{\hat{a}_{i}}\right]\right\}, \quad i=1, \ldots, n,
$$

so that simply all scalar integers within the given interval have to be selected. The normalized length $2 \lambda$ of the search interval does not depend on the variance of the components of the float solution $\hat{\boldsymbol{a}}$, if $\lambda$ is chosen such that $P\left(\left|\hat{a}_{i}-a\right|^{2} / \sigma_{\hat{a}_{i}}^{2}<\lambda^{2}\right)=P\left(a_{i} \in \Theta_{\hat{a}_{i}}^{\lambda}\right)=1-\alpha$ with a constant value of $\alpha$, where we use that $\left|\hat{a}_{i}-a\right|^{2} / \sigma_{\hat{a}_{i}}^{2}$ follows a central $\chi^{2}$ distribution with one degree of freedom.

\subsubsection{Sequential Scalar Approximation}

In the previous section it is shown that the best integer equivariant estimate $\hat{\boldsymbol{a}}_{\mathrm{BIE}}$ and the parallel scalar approximation $\hat{\boldsymbol{a}}_{\mathrm{PBIE}}$ thereof can in a way be interpreted as the counterparts of $\check{\boldsymbol{a}}_{\mathrm{ILS}}$ and $\check{\boldsymbol{a}}_{\mathrm{IR}}$ from the class of integer estimators. The logical consequence is therefore to also formulate the counterpart of integer bootstrapping $\check{\boldsymbol{a}}_{\mathrm{IB}}$, i.e., to combine the principle of best integer equivariant estimation with a sequential processing strategy, in which the estimates of the previous elements 
are taken into account through the correlation between the elements of $\hat{\boldsymbol{a}}$ (Brack et al. 2013, 2014). Starting with the last entry and proceeding in reversed order, the elements of $\hat{\boldsymbol{a}}_{\mathrm{SBIE}}$ are defined as

$$
\hat{a}_{\mathrm{SBIE}, i}=\sum_{z \in \mathbb{Z}} z \cdot \frac{\exp \left(-\frac{1}{2 \sigma_{\hat{a}_{i \mid I}^{2}}^{2}}\left|\hat{a}_{i \mid I}-z\right|^{2}\right)}{\sum_{u \in \mathbb{Z}} \exp \left(-\frac{1}{2 \sigma_{\hat{a}_{i \mid I}^{2}}^{2}}\left|\hat{a}_{i \mid I}-u\right|^{2}\right)}=\sum_{z \in \mathbb{Z}} z \cdot w_{z}\left(\hat{a}_{i \mid I}\right) .
$$

These sequential scalar estimates are almost identical to the parallel scalar estimates $\hat{a}_{\mathrm{PBIE}, i}$, cf. (7.9), but the elements $\hat{a}_{i}$ of the float estimate are replaced by the conditional estimates $\hat{a}_{i \mid I}$ with $I=\{i+1, \ldots, n\}$. They are given by

$$
\hat{a}_{i \mid I}=\hat{a}_{i}-\sum_{j=i+1}^{n} \sigma_{\hat{a}_{i} \hat{a}_{j \mid J}} \sigma_{\hat{a}_{j \mid J}}^{-2}\left(\hat{a}_{j \mid J}-\hat{a}_{\mathrm{SBIE}, j}\right) .
$$

The coefficients in (7.17) and (7.18) required to compute the elements of sequential scalar approximation $\hat{\boldsymbol{a}}_{\mathrm{SBIE}}$ are available from the triangular decomposition $\boldsymbol{Q}_{\hat{\boldsymbol{a}}}=\boldsymbol{L}^{\mathrm{T}} \boldsymbol{D} \boldsymbol{L}$ of the float covariance matrix, see Section 3.1.3. The estimate $\hat{\boldsymbol{a}}_{\mathrm{SBIE}}$ becomes identical to the integer bootstrapping solution $\check{\boldsymbol{a}}_{\mathrm{IB}}$, if the weighted sum of scalar integers (7.17) is replaced by simple rounding $\left\lfloor\hat{a}_{i \mid I}\right\rceil$. The corresponding estimate $\hat{\boldsymbol{b}}_{\mathrm{SBIE}}$ of the real valued parameters $\boldsymbol{b}$ is computed as stated in (7.7), where $\hat{\boldsymbol{a}}_{\mathrm{BIE}}$ is replaced by $\hat{\boldsymbol{a}}_{\mathrm{SBIE}}$. A shift of the float solution $\hat{\boldsymbol{a}}$ by an arbitrary integer results in the same shift of $\hat{\boldsymbol{a}}_{\mathrm{SBIE}}$. With the definition of $\hat{\boldsymbol{b}}_{\mathrm{SBIE}}$, it is therefore easily verified that the estimate $\hat{\theta}_{\mathrm{SBIE}}=\boldsymbol{l}_{\boldsymbol{a}}^{\mathrm{T}} \hat{\boldsymbol{a}}_{\mathrm{SBIE}}+\boldsymbol{l}_{\boldsymbol{b}}^{\mathrm{T}} \hat{\boldsymbol{b}}_{\mathrm{SBIE}}$ of $\theta$ with arbitrary vectors of coefficients is a valid integer equivariant estimator as defined in (7.1). The pdf of $\hat{\boldsymbol{a}}_{\mathrm{SBIE}}$ is shown in Figure 7.1 for a two dimensional example. It is very similar to that of $\hat{\boldsymbol{a}}_{\mathrm{PBIE}}$, but the estimates of the first element are slightly more concentrated around the integer values.

The elements of $\hat{\boldsymbol{a}}_{\mathrm{SBIE}}$ can be interpreted in a similar way as the elements of $\hat{\boldsymbol{a}}_{\mathrm{PBIE}}$ in the previous section. The difference is that not the float estimate $\hat{a}_{i}$ itself is pulled towards the closest integer $\left\lfloor\hat{a}_{i}\right\rceil$, but rather the conditional estimate $\hat{a}_{i \mid I}$ is pulled towards $\left\lfloor\hat{a}_{i \mid I}\right\rceil$. Following the respective derivations in Section 7.2.2 we can again analyze $\hat{\boldsymbol{a}}_{\mathrm{SBIE}}$ for very low and very high precision of $\hat{\boldsymbol{a}}$. Combining (7.11) with (7.18) and the sequential processing strategy shows that if $\hat{\boldsymbol{a}}$ is of very low precision, then $\hat{\boldsymbol{a}}_{\mathrm{SBIE}}$ also converges to the float solution $\hat{\boldsymbol{a}}$. If $\hat{\boldsymbol{a}}$ is of very high precision with $\sigma_{\hat{a}_{i \mid I}} \rightarrow 0, i=1, \ldots n$, we can find that-analogously to the discussion in the previous section-the weights $w_{z}\left(\hat{a}_{i \mid I}\right)$ assume the binary values $\lim _{{\hat{\hat{a}_{i \mid I}}} \rightarrow 0} w_{\left\lfloor\hat{a}_{i \mid I}\right\rceil}\left(\hat{a}_{i \mid I}\right)=1$ and $\lim _{\hat{\sigma}_{\hat{a}_{i \mid I} \rightarrow 0}} w_{z}\left(\hat{a}_{i \mid I}\right)=0, \forall z \in \mathbb{Z} \backslash\left\lfloor\hat{a}_{i \mid I}\right\rceil$. Applying this result to (7.17) shows that $\lim _{\sigma_{\hat{a}_{i \mid I} \rightarrow 0}} \hat{a}_{\mathrm{SBIE}, i}=\left\lfloor\hat{a}_{i \mid I}\right\rceil$, so that $\hat{\boldsymbol{a}}_{\mathrm{SBIE}}$ converges to the integer bootstrapping solution $\check{\boldsymbol{a}}_{\mathrm{IB}}$ if $\hat{\boldsymbol{a}}$ is of very high precision. The sequential scalar best integer equivariant approximation can thus indeed be interpreted as the sought for counterpart of integer bootstrapping. If $\boldsymbol{Q}_{\hat{a}}$ is diagonal, the sequential estimation scheme used to compute $\hat{\boldsymbol{a}}_{\mathrm{SBIE}}$ is identical to a separate estimation of each element and therefore optimal, see Section 7.2.2. As the estimates $\hat{\boldsymbol{a}}_{\mathrm{SBIE}}$ and $\hat{\boldsymbol{b}}_{\mathrm{SBIE}}$ depend on the parameterization of $\hat{\boldsymbol{a}}$ via the integer transformation $Z, \hat{\boldsymbol{a}}_{\mathrm{SBIE}}$ should be computed in a decorrelated ambiguity space.

When implementing the sequential scalar approximation of the best integer equivariant estimator, the infinite sum in (7.17) has to be replaced with a sum over a finite set, which should be chosen as the set of all integers within a certain interval around the conditional float solution $\hat{a}_{i \mid I}$ from (7.18) as $\Theta_{\hat{a}_{i \mid I}^{\lambda}}^{\lambda}=\left\{z \in \mathbb{Z} \mid z \in\left[\hat{a}_{i \mid I}-\lambda \sigma_{\hat{a}_{i \mid I}}, \hat{a}_{i \mid I}+\lambda \sigma_{\hat{a}_{i \mid I}}\right]\right\}, i=1, \ldots, n$, see (7.16). 


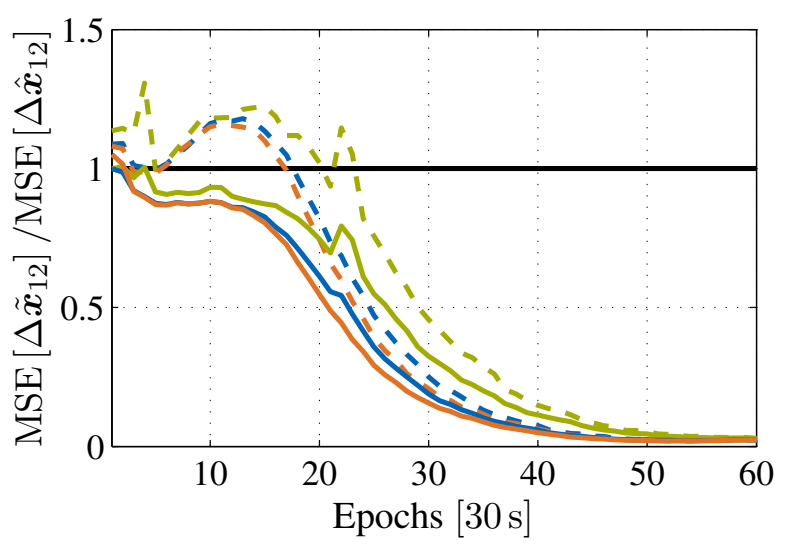

Figure 7.4: Ratio between the mean squared error of the estimated user position $\Delta \tilde{\boldsymbol{x}}_{12}$ and the mean squared error of the float solution $\Delta \hat{\boldsymbol{x}}_{12}$; solid orange, blue, and green lines represent the best integer equivariant estimator, the sequential scalar approximation, and the parallel scalar approximation; dashed orange, blue, and green lines represent the ambiguity fixed solutions from integer least-squares, integer bootstrapping, and element-wise rounding.

\subsection{GNSS Positioning Performance}

A basic qualitative assessment of the positioning capabilities of the best integer equivariant estimator and the two scalar approximations as compared to the float solution and different ambiguity fixed solutions is presented for a long baseline example. The ambiguity estimates $\hat{\boldsymbol{a}}_{\mathrm{PBIE}}$ and $\hat{\boldsymbol{a}}_{\mathrm{SBIE}}$ are computed in the LAMBDA decorrelated space, and integer rounding and integer bootstrapping are also applied after the LAMBDA decorrelation.

The first quality measure that is analyzed is the mean square error of the estimate $\Delta \tilde{\boldsymbol{x}}_{12}$ of the incremental user position $\Delta \boldsymbol{x}_{12} \in \mathbb{R}^{3}$

$$
\operatorname{MSE}\left[\Delta \tilde{\boldsymbol{x}}_{12}\right]=\mathrm{E}\left[\left(\Delta \boldsymbol{x}_{12}-\Delta \tilde{\boldsymbol{x}}_{12}\right)^{\mathrm{T}}\left(\Delta \boldsymbol{x}_{12}-\Delta \tilde{\boldsymbol{x}}_{12}\right)\right],
$$

where $\Delta \tilde{\boldsymbol{x}}_{12}$ is used to denote the part of the ambiguity fixed solutions $\check{\boldsymbol{b}}$ that result from integer rounding, integer bootstrapping, and integer least-squares, or the part of the integer equivariant estimates $\hat{\boldsymbol{b}}_{\mathrm{BIE}}, \hat{\boldsymbol{b}}_{\mathrm{PBIE}}$, and $\hat{\boldsymbol{b}}_{\mathrm{SBIE}}$ that correspond to $\Delta \boldsymbol{x}_{12} \in \mathbb{R}^{3}$. Since all these estimators are unbiased, the mean squared error is identical to the trace of the variance: $\operatorname{MSE}\left[\Delta \tilde{\boldsymbol{x}}_{12}\right]=$ $\operatorname{tr}\left(\operatorname{Var}\left[\Delta \tilde{\boldsymbol{x}}_{12}\right]\right)$. The mean squared error of the float solution $\Delta \hat{\boldsymbol{x}}_{12}$ is given by MSE $\left[\Delta \hat{\boldsymbol{x}}_{12}\right]=$ $\operatorname{tr}\left(\operatorname{Var}\left[\Delta \hat{\boldsymbol{x}}_{12}\right]\right)=\operatorname{tr}\left(\boldsymbol{Q}_{\Delta \hat{\boldsymbol{x}}_{12}}\right)$, with $\boldsymbol{Q}_{\Delta \hat{\boldsymbol{x}}_{12}}$ the part of $\boldsymbol{Q}_{\hat{\boldsymbol{b}}}$ that corresponds to $\Delta \hat{\boldsymbol{x}}_{12}$.

The mean square errors $\operatorname{MSE}\left[\Delta \tilde{\boldsymbol{x}}_{12}\right]$ relative to the mean square error of the float estimate are shown in Figure 7.4 as a function of the number of epochs. The best integer equivariant estimates in solid orange are mean square error optimal, thus resulting in the smallest values. The results of the sequential scalar approximation in solid blue are slightly worse compared to the best integer equivariant estimator due to remaining correlations between the elements of $\hat{\boldsymbol{a}}$, and the results of the parallel scalar approximation in solid green are again inferior. In the first epoch, when the precision of the float ambiguity estimate $\hat{\boldsymbol{a}}$ is very low, the parallel and sequential scalar best integer equivariant schemes are-as expected-identical to the float solution, i.e., they result in a mean square error ratio of one. The reason why the optimal best integer equivariant estimates appear to have a too large mean square error in the first epochs is the rough approximation of $\hat{\boldsymbol{a}}_{\mathrm{BIE}}$ via a too small search space $\Theta_{\hat{a}}^{\lambda}$. The mean square error results with integer least-squares, integer bootstrapping, and element-wise rounding are shown in dashed orange, blue, and green. They are always inferior to their integer equivariant counterparts. In the first $\sim 20$ epochs, when the respective failure rates are too large, the mean square errors of the resulting estimates of the user position exceed the mean square error of the float solution. With increasing number of epochs and therefore increasing precision of $\hat{\boldsymbol{a}}$, the estimates $\hat{\boldsymbol{a}}_{\mathrm{BIE}}, \hat{\boldsymbol{a}}_{\mathrm{SBIE}}$, and $\hat{\boldsymbol{a}}_{\mathrm{PBIE}}$ converge to $\check{\boldsymbol{a}}_{\mathrm{ILS}}, \check{\boldsymbol{a}}_{\mathrm{IB}}$, and $\check{\boldsymbol{a}}_{\mathrm{IR}}$, i.e., the gaps between the solid and dashed lines of identical color approach zero. With vanishing 


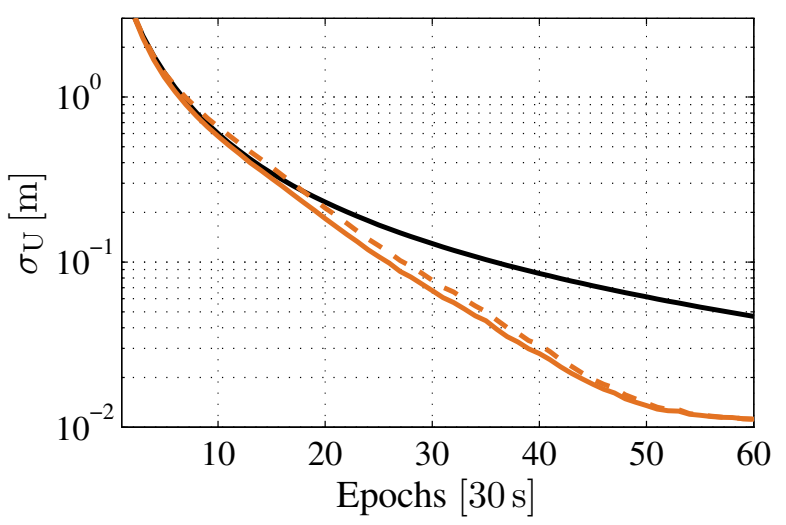

Figure 7.5: Standard deviation of up component of estimated user position; black: float solution, solid orange: best integer equivariant solution, dashed orange: fixed solution with integer least-squares.

failure rate, the ambiguity fixed solutions are eventually identical to the best integer equivariant solution. The peaks of the mean square error of the parallel scalar best integer equivariant approximation and element-wise rounding after 4 and 22 epochs can be explained by the dependency of the estimates on the integer transformation, which can of course change over time when using the LAMBDA decorrelation. The influence of the integer transformation appears to be much smaller in the present example when using the sequential ambiguity estimation schemes (blue lines).

The stochastic characteristics of the positioning solutions are now investigated in more detail for the float solution, the ambiguity fixed solution with integer least-squares, and the best integer equivariant solution. The scalar approximations of the best integer equivariant estimator and other ambiguity fixed solutions, including partially fixed solutions, can be interpreted similarly. By definition, the best integer equivariant estimator minimizes the mean square error of any linear combination of the parameters within the class of integer equivariant estimators, e.g., the mean square error of the east, north, and up coordinate estimates. The float and fixed solution with integer least-squares are unbiased, so that this also holds for the respective standard deviations, which is shown at the example of the up component in Figure 7.5. The interpretation of this figure is very similar to Figure 7.4, with the difference that now the improvement of the float solution over time is shown explicitly. One advantage of the best integer equivariant estimator over the float and any (partially) fixed solutions is that the question of when to fix which subset $\mathcal{I}$ of ambiguities is avoided, as the best integer equivariant estimator automatically converges to the float and fully fixed solutions for very low and very high precision of $\hat{\boldsymbol{a}}$, respectively, and is always the best solution in terms of the mean square error.

Similar values of the mean square error do not imply that the stochastic properties of the coordinate estimates are also similar, which is shown in Figure 7.6 by means of the cumulative distribution of the positioning errors $\left\|\Delta \boldsymbol{x}_{12}-\Delta \hat{\boldsymbol{x}}_{12}\right\|$ and $\left\|\Delta \boldsymbol{x}_{12}-\Delta \tilde{\boldsymbol{x}}_{12}\right\|$ after 15 and 35 epochs. The results of the fixed solution show what has been observed earlier in this dissertation. Correct integer estimates, which occur with a probability of $15.1 \%$ after 15 epochs and $90.3 \%$ after 35 epochs, lead to very precise coordinate estimates, whereas incorrect integer estimates generally result in large positioning errors that can also exceed the error of the float solution. The best integer equivariant estimator can be interpreted as a trade off between the float and fully fixed solution. It does not produce hard integer estimates, which means that it is less likely to have very small positioning errors that result with high probability from correct integer estimates, but also that large positioning errors that result with high probability from incorrect integer estimates are less likely. 


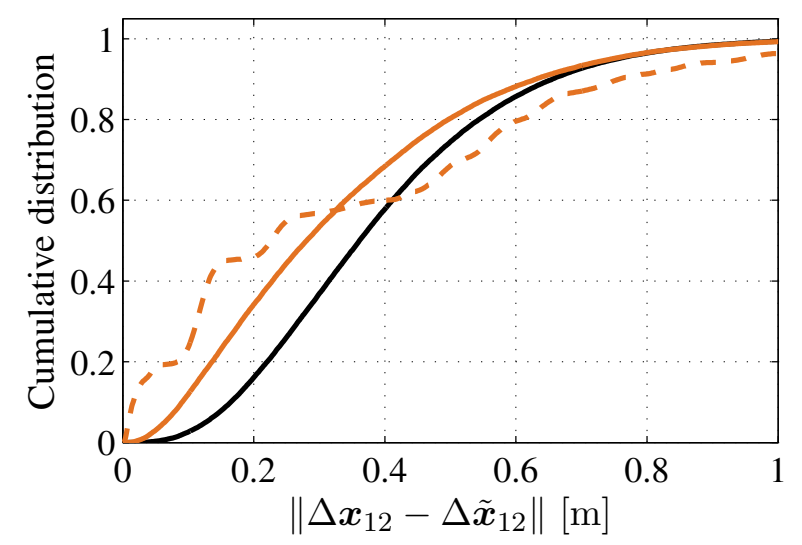

(a) After 15 epochs

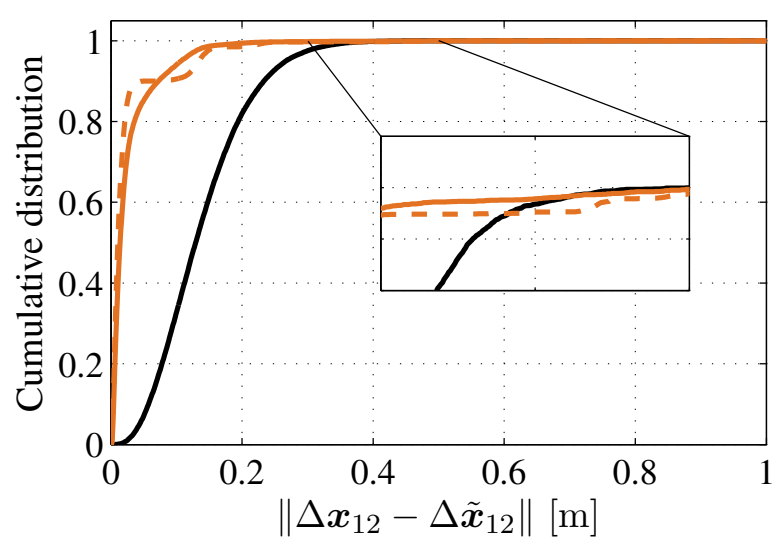

(b) After 35 epochs

Figure 7.6: Cumulative distribution of positioning error; black: float solution, solid orange: best integer equivariant solution, dashed orange: fixed solution with integer least-squares. 



\section{Summary and Conclusions}

The objective of this dissertation was the development of theory, methods, and algorithms for selecting and resolving a subset of the integers in linear models with integer or mixed integer and real-valued parameters, in particular with the application of partial carrier-phase ambiguity resolution for GNSS positioning in mind. The most important results can be summarized as follows.

- Before resolving a subset of the GNSS integer ambiguities, a valid parameterization by means of a prior transformation with matrix $Z$ has to be chosen, which defines the set of linear ambiguity combinations that can be estimated. This transformation can completely change the problem of partial ambiguity resolution, and therefore also for instance the success rate and the impact on the quality of the partially ambiguity fixed estimates of the real valued parameters. It was demonstrated that resolving a subset of the highly correlated original double difference ambiguities can often be almost equally hard as resolving the full set. Decorrelation transformations are therefore very helpful in order to enable a true benefit of partial ambiguity resolution.

- It is well known that integer least-squares is optimal in the sense of maximizing the probability of correctly resolving the full set of integer parameters in linear observation models with additive Gaussian noise. For resolving an arbitrary deterministic subset of integer parameters, however, integer least-squares is no longer the optimal choice. The optimal estimator leading to the highest possible probability of correctly resolving the given subset was derived. It contains an infinite sum over integer vectors and can be implemented by means of two consecutive searches. Numerical examples showed that for GNSS ambiguity resolution a very good suboptimal strategy is to compute the full dimensional integer least-squares solution and to select those components thereof, which correspond to the given subset. The success rates of this strategy are close to optimal, while only the-comparably simple-computation of an integer least-squares solution is required.

- One option to select the ambiguity subset to be resolved is to only consider the description of the underlying system model and to evaluate measures like the ambiguity failure rate and the partially fixed precision of the real valued parameters as given by the conditional covariance matrix for different ambiguity subsets. With a maximum failure rate constraint, the optimal subset can be defined as the one that leads to the best partially fixed precision among all subsets for which the failure rate constraint is met. Due to the sheer number of possible subsets, finding the so defined optimal subset might not be a practical strategy, especially when the number of ambiguities is large. A very simple yet clearly suboptimal selection strategy is the use of the failure rate of truncated integer bootstrapping with proper ordering of the ambiguity parameters.

- Including the measurement data when selecting the subset of integer parameters to be resolved makes the subset itself a discrete random variable. Such an estimator can for example be defined such that either a single specific subset of the integer parameters is resolved or none of them is. The optimal estimator of this form leading to the highest probability of resolving the given subset under a failure rate constraint and a computationally simpler difference test 
based estimator were formulated. The disadvantage of these schemes, potentially limiting their practical applicability, is that again a specific subset has to be a priori selected.

- The probably most relevant results of this dissertation for GNSS practice are the per element acceptance tests, in particular the per element difference test. The full integer least-squares solution is computed and each scalar component thereof is tested for acceptance using the respective best counter-hypothesis. Since these schemes only require a single integer solutionthe full dimensional integer least-squares solution-they can be very efficiently implemented, e.g., through a slight modification of the LAMBDA method. By adjusting a single critical value, a fixed failure rate can be guaranteed. The subset of integers to be resolved automatically results from the acceptance tests without the need to a priori select possible options. The numerical simulation results show that-without employing any information about the aimed at precision-the per element difference test achieves excellent GNSS positioning results. A conservative functional description was shown to be a suitable tool for a user friendly description of the fixed failure rate critical value.

- In various simulated GNSS positioning cases the benefit of partial ambiguity resolution techniques were demonstrated. In particular, the simulations showed that the convergence time for long baseline positioning can be expected to be clearly reduced, and the range of instantaneous RTK positioning considerably extended. It was shown that partial ambiguity resolution proves particularly useful for future multi-frequency, multi-GNSS solutions, when the number of ambiguity parameters is large. These findings were verified with real GPS+BDS data.

- A parallel and a sequential scalar approximation of the best integer equivariant estimator were formulated. They lead to slightly suboptimal mean square error results, while the computational complexity is drastically reduced. 


\section{Appendix}

\section{A1. S-System Theory}

We consider a system of linear equations in the form

$$
\boldsymbol{y}=\boldsymbol{A} \boldsymbol{x}+\boldsymbol{\eta},
$$

with $\boldsymbol{y} \in \mathbb{R}^{q}$ the measurement vector, $\boldsymbol{x} \in \mathbb{R}^{p}$ the vector of unknowns, $\boldsymbol{A} \in \mathbb{R}^{q \times p}$ the design matrix with $\operatorname{rank}(\boldsymbol{A})=r<p$, and $\boldsymbol{\eta} \in \mathbb{R}^{q}$ a zero-mean additive noise vector. Let the linear estimator $\hat{\boldsymbol{x}}$ of the parameter vector $\boldsymbol{x}$ be given by

$$
\hat{\boldsymbol{x}}=\boldsymbol{T y}
$$

This estimator is unbiased, if

$$
E[\hat{\boldsymbol{x}}]=\boldsymbol{T} \boldsymbol{A} \boldsymbol{x}=\boldsymbol{x},
$$

i.e., if $\boldsymbol{T} \boldsymbol{A}=\boldsymbol{I}_{p}$. This criterion, however, cannot be fulfilled, since the rank of a product of matrices can never exceed the rank of either factor. Consequently, the parameter vector of $\boldsymbol{x}$ is not unbiased estimable, but certain linear combination of the elements of $\boldsymbol{x}$ are. In this section, the aspects of S-system theory (Baarda 1973; Teunissen 1984; Odijk et al. 2016) relevant to cope with this problem are introduced.

The rank deficiency of $\boldsymbol{A}$ is of the $\operatorname{size} \operatorname{dim} \mathcal{N}(\boldsymbol{A})=p-r$, where $\mathcal{N}(\cdot)$ is used to denote the null space. Let $\boldsymbol{V} \in \mathbb{R}^{p \times(p-r)}$ contain a set of basis vectors for $\mathcal{N}(\boldsymbol{A})$, so that $\boldsymbol{A} \boldsymbol{V}=\mathbf{0}$. Note that the null space $\mathcal{N}(\boldsymbol{A})$ is unique, but the basis vectors in $\boldsymbol{V}$ are not. Further, let $\boldsymbol{S} \in \mathbb{R}^{p \times r}$ contain a set of basis vectors so that $\mathcal{R}(\boldsymbol{S}) \oplus \mathcal{R}(\boldsymbol{V})=\mathbb{R}^{n}$, where $\mathcal{R}(\cdot)$ is used to denote the range space of a matrix and $\oplus$ denotes the direct $\operatorname{sum}(\mathcal{R}(\boldsymbol{S}) \cap \mathcal{R}(\boldsymbol{V})=\{\boldsymbol{0}\})$. There are infinitely many options to chose the basis vectors in $\boldsymbol{S}$. We can now decompose the parameter vector $\boldsymbol{x}$ as

$$
\boldsymbol{x}=\underbrace{\boldsymbol{S \alpha}}_{x_{S}}+\underbrace{\boldsymbol{V} \boldsymbol{\beta}}_{x_{V}}=[\boldsymbol{S}, \boldsymbol{V}]\left[\begin{array}{l}
\boldsymbol{\alpha} \\
\boldsymbol{\beta}
\end{array}\right],
$$

where $\boldsymbol{x}_{\boldsymbol{S}} \in \mathcal{R}(\boldsymbol{S})$ denotes the estimable part of $\boldsymbol{x}$ and $\boldsymbol{x}_{\boldsymbol{V}} \in \mathcal{R}(\boldsymbol{V})$ the inestimable part of $\boldsymbol{x}$. Substituting (A4) into the rank deficient system model (A1) leads to the full rank system model

$$
\boldsymbol{y}=\boldsymbol{A} \boldsymbol{x}+\boldsymbol{\eta}=\boldsymbol{A}(\boldsymbol{S} \boldsymbol{\alpha}+\boldsymbol{V} \boldsymbol{\beta})+\boldsymbol{\eta}=(\boldsymbol{A} \boldsymbol{S}) \boldsymbol{\alpha}+\boldsymbol{\eta},
$$

with the unbiased estimable vector of unknowns $\boldsymbol{\alpha} \in \mathbb{R}^{r}$ and the design matrix $\boldsymbol{A} \boldsymbol{S} \in \mathbb{R}^{q \times r}$. We can now replace the original system model (A1) with (A5) and estimate the new parameter vector $\boldsymbol{\alpha}$. It is of importance, which combinations of the original parameters $\boldsymbol{x}$ are represented by $\boldsymbol{\alpha}$, which depends on the choice of $\boldsymbol{S}$. Depending on the application at hand, $\boldsymbol{S}$ should be carefully chosen, since often not all options make sense. As an example, if we want to estimate carrierphase ambiguities as integers, we have to make sure that the corresponding entries in $\alpha$ are only combinations of integer terms and are not combined with other non-integer bias parameters. Since $[\boldsymbol{S}, \boldsymbol{V}] \in \mathbb{R}^{p \times p}$ is a full rank square matrix, (A4) can be solved for $\boldsymbol{\alpha}$ and $\boldsymbol{\beta}$ :

$$
\left[\begin{array}{c}
\boldsymbol{\alpha} \\
\boldsymbol{\beta}
\end{array}\right]=[\boldsymbol{S}, \boldsymbol{V}]^{-1} \boldsymbol{x}=\left[\begin{array}{l}
{\left[\boldsymbol{V}^{\perp, \mathrm{T}} \boldsymbol{S}\right]^{-1} \boldsymbol{V}^{\perp, \mathrm{T}}} \\
{\left[\boldsymbol{S}^{\perp, \mathrm{T}} \boldsymbol{V}\right]^{-1} \boldsymbol{S}^{\perp, \mathrm{T}}}
\end{array}\right] \boldsymbol{x} .
$$


The matrix $\boldsymbol{S}^{\perp} \in \mathbb{R}^{p \times(p-r)}$ contains a set of basis vectors spanning the subspace orthogonal to $\mathcal{R}(\boldsymbol{S})$, i.e., so that $\boldsymbol{S}^{\perp, \mathrm{T}} \boldsymbol{S}=\mathbf{0}$. The matrix $\boldsymbol{V}^{\perp} \in \mathbb{R}^{p \times r}$ is defined analogously for $\mathcal{R}(\boldsymbol{V})$ instead of $\mathcal{R}(\boldsymbol{S}) . \boldsymbol{S}^{\perp, \mathrm{T}} \boldsymbol{x}$ is called the S-basis and defines the constraints that are required to solve for the estimable parameters.

The steps necessary to solve a rank deficient system of linear equations can be summarized as:

1) Identification of the rank deficiencies of $\boldsymbol{A}$ /the null space $\mathcal{N}(\boldsymbol{A})$. This can be done by analyzing which parameter changes leave the observations $y$ invariant.

2) $p-r$ constraints have to be added in the form of $\boldsymbol{S}^{\perp, \mathrm{T}} \boldsymbol{x}$, with $\boldsymbol{S}^{\perp} \in \mathbb{R}^{p \times(p-r)}$. The constraints have to be formulated such that $\mathcal{R}(\boldsymbol{S}) \oplus \mathcal{N}(\boldsymbol{A})=\mathbb{R}^{p}$.

3) Choosing a basis matrix $\boldsymbol{S}$ defines the full rank system of equations (A5).

4) A mathematical/physical interpretation of the estimable parameters $\boldsymbol{\alpha}$, which are linked to the original parameters $\boldsymbol{x}$ via (A6), can now be given.

A different choice of the S-system leads to a different set of estimable parameters. One might, however, be interested in comparing estimates resulting from different S-systems. With (A4) and (A6) we can link the estimable part $\boldsymbol{x}_{S}$ of the parameter vector to the parameter vector $\boldsymbol{x}$ itself via

$$
\begin{aligned}
\boldsymbol{x}_{\boldsymbol{S}} & =\boldsymbol{S}\left[\boldsymbol{V}^{\perp, \mathrm{T}} \boldsymbol{S}\right]^{-1} \boldsymbol{V}^{\perp, \mathrm{T}} \boldsymbol{x}=\boldsymbol{I}_{p}-\boldsymbol{V}\left[\boldsymbol{S}^{\perp, \mathrm{T}} \boldsymbol{V}\right]^{-1} \boldsymbol{S}^{\perp, \mathrm{T}} \boldsymbol{x} \\
& =\boldsymbol{S} \boldsymbol{x} .
\end{aligned}
$$

The transformation matrix $\mathcal{S} \in \mathbb{R}^{p \times p}$ is referred to as the S-transformation. It is easily verified that $\mathcal{S} \boldsymbol{V}=\mathbf{0}, \mathcal{S} \boldsymbol{S}=\boldsymbol{S}$, and $\mathcal{S} \mathcal{S}=\mathcal{S}$, i.e., $\mathcal{S}$ is a projector that projects onto $\mathcal{R}(\boldsymbol{S})$ and along $\mathcal{N}(\boldsymbol{A})$. Note that $\mathcal{S}$ does not depend on the matrix $\boldsymbol{S}$, but only on the subspaces $\mathcal{R}(\boldsymbol{S})$ and $\mathcal{N}(\boldsymbol{A})$. The choice of $\boldsymbol{S}$ does, however, affect the parameter functions $\boldsymbol{\alpha}$ that are estimated in (A5). Let $\mathcal{S}^{\prime}$ denote the $\mathrm{S}$-transformation of a different $S$-system based on the set of basis vectors in $\boldsymbol{S}^{\prime}$. As already mentioned, we cannot directly compare estimates of the parameter vectors or the quality thereof which are based on different $\mathrm{S}$-systems, since they represent different estimable functions. But since

$$
\mathcal{S S}^{\prime}=\mathcal{S}
$$

and therefore

$$
\boldsymbol{x}_{\boldsymbol{S}}=\mathcal{S} \boldsymbol{x}=\mathcal{S S}^{\prime} \boldsymbol{x}=\mathcal{S} \boldsymbol{x}_{\boldsymbol{S}^{\prime}}
$$

we can easily transform parameter estimates from one S-system to another. Let $\boldsymbol{\alpha}^{\prime}$ be the estimable parameter vector under the S-system of $\boldsymbol{S}^{\prime}$ and let $\hat{\boldsymbol{\alpha}}^{\prime}$ be an estimate of $\boldsymbol{\alpha}^{\prime}$. We can then transform the estimate $\hat{\boldsymbol{x}}_{\boldsymbol{S}^{\prime}}=\boldsymbol{S}^{\prime} \hat{\boldsymbol{\alpha}}^{\prime}$ for the estimable part $\boldsymbol{x}_{\boldsymbol{S}^{\prime}}$ of $\boldsymbol{x}$ in the S-system of $\boldsymbol{S}^{\prime}$ to the S-system of $S$ via

$$
\hat{\boldsymbol{x}}_{\boldsymbol{S}}=\mathcal{S} \hat{\boldsymbol{x}}_{\boldsymbol{S}^{\prime}}
$$

without even requiring knowledge about the original S-system as defined by $\boldsymbol{S}^{\prime}$. All S-systems can be linked to one another by applying an S-transformation.

\section{A2. Sufficient Statistics}

We consider the problem of estimating the parameter vector $\boldsymbol{x}$ from the random vector of observations $\boldsymbol{y}$. The concept of a sufficient statistic is to find a statistic $\boldsymbol{t}=f(\boldsymbol{y})$ that contains all the information about $\boldsymbol{x}$ that can be found in the original vector of observations $\boldsymbol{y}$. The parameter vector $\boldsymbol{x}$ can therefore equivalently be estimated based on $\boldsymbol{t}$ instead of $\boldsymbol{y}$. Let $p_{\boldsymbol{y}}(\boldsymbol{\psi} ; \boldsymbol{x})$ denote the 
pdf of the observation vector $\boldsymbol{y}$, in which the dependency on the non-random parameter vector $\boldsymbol{x}$ is made explicit. The statistic $t$ is sufficient for the parameter vector $\boldsymbol{x}$, if

$$
p_{\boldsymbol{y} \mid \boldsymbol{t}}(\boldsymbol{\psi} \mid \boldsymbol{\tau} ; \boldsymbol{x})=p_{\boldsymbol{y} \mid \boldsymbol{t}}(\boldsymbol{\psi} \mid \boldsymbol{\tau}),
$$

i.e., if the pdf of the vector of observations $y$ given the statistic $t$ is independent of the parameter vector $\boldsymbol{x}$ (Kay 1993; Poor 1994). In case the parameter vector $\boldsymbol{x}$ is also random, the condition corresponding to (A11) reads $p_{\boldsymbol{y} \mid \boldsymbol{t}, \boldsymbol{x}}(\boldsymbol{\psi} \mid \boldsymbol{\tau}, \boldsymbol{\chi})=p_{\boldsymbol{y} \mid \boldsymbol{t}}(\boldsymbol{\psi} \mid \boldsymbol{\tau})$. It is clear that any invertible function $f(\cdot)$ produces a sufficient statistic, but is also generally not very helpful since one can then also work with the original vector of observations $y$. The goal when using a sufficient statistic is usually to reduce the observations as much as possible without destroying information about $\boldsymbol{x}$. Such a statistic $\boldsymbol{t}$ is called a minimal sufficient statistic and is of minimum dimension among all sufficient statistics.

The question is now how to find a (minimal) sufficient statistic. The Fisher-Neyman factorization theorem states that a statistic $\boldsymbol{t}=f(\boldsymbol{y})$ is sufficient for $\boldsymbol{x}$, if there exist functions $a(\cdot)$ and $b(\cdot)$ such that

$$
p_{\boldsymbol{y}}(\boldsymbol{\psi} ; \boldsymbol{x})=a(f(\boldsymbol{\psi}), \boldsymbol{x}) b(\boldsymbol{\psi}) .
$$

If a minimal sufficient statistic exists, which is not always the case, then every complete sufficient statistic is necessarily minimal, where completeness can be shown as follows. Let $\boldsymbol{t}=f(\boldsymbol{y})$ be a sufficient statistic for $\boldsymbol{x}$, to which an arbitrary function $k(\boldsymbol{t})$ is applied. If $\mathrm{E}[k(\boldsymbol{t})]=\mathbf{0}$ for every possible value of $\boldsymbol{x}$ implies that $P(k(\boldsymbol{t})=\mathbf{0})=1$ for all $\boldsymbol{x}$, then $\boldsymbol{t}$ is complete. Given that the pdf $p_{\boldsymbol{y}}(\boldsymbol{\psi} ; \boldsymbol{x})$ is a member of the family of exponential distributions, i.e., it can be written as

$$
p_{\boldsymbol{y}}(\boldsymbol{\psi} ; \boldsymbol{x})=c(\boldsymbol{x}) b(\boldsymbol{\psi}) \exp \left(g(\boldsymbol{x})^{\mathrm{T}} f(\boldsymbol{\psi})\right)
$$

then $\boldsymbol{t}=f(\boldsymbol{y})$ is a sufficient statistic for $\boldsymbol{x}$ according to (A12). It is a complete sufficient statistic for $\boldsymbol{x}$, and therefore usually also a minimal sufficient statistic, if $f(\cdot)$ is of minimal dimension.

We can now discuss the linear observation model of the form $\boldsymbol{y}=\boldsymbol{A x}+\boldsymbol{\eta}$ with the vector of unknowns $\boldsymbol{x}$ and the additive Gaussian noise $\boldsymbol{\eta} \sim \mathcal{G}(\mathbf{0}, \boldsymbol{Q})$, so that

$$
\begin{aligned}
p_{\boldsymbol{y}}(\boldsymbol{\psi} ; \boldsymbol{x}) & =\frac{1}{\sqrt{\operatorname{det}(2 \pi \boldsymbol{Q})}} \exp \left(-\frac{1}{2}(\boldsymbol{\psi}-\boldsymbol{A} \boldsymbol{x})^{\mathrm{T}} \boldsymbol{Q}^{-1}(\boldsymbol{\psi}-\boldsymbol{A} \boldsymbol{x})\right) \\
& =\underbrace{\frac{1}{\sqrt{\operatorname{det}(2 \pi \boldsymbol{Q})}} \exp \left(-\frac{1}{2} \boldsymbol{x}^{\mathrm{T}} \boldsymbol{A}^{\mathrm{T}} \boldsymbol{Q}^{-1} \boldsymbol{A} \boldsymbol{x}\right)}_{c(\boldsymbol{x})} \underbrace{\exp \left(-\frac{1}{2} \boldsymbol{\psi}^{\mathrm{T}} \boldsymbol{Q}^{-1} \boldsymbol{\psi}\right)}_{b(\boldsymbol{\psi})} \exp \left(\boldsymbol{x}^{\mathrm{T}} \boldsymbol{A}^{\mathrm{T}} \boldsymbol{Q}^{-1} \boldsymbol{\psi}\right) .
\end{aligned}
$$

The vector of observations $\boldsymbol{y}$ follows an exponential distribution, where the first two factors $c(\boldsymbol{x})$ and $b(\boldsymbol{\psi})$ of expression (A13) are easily identified in (A14). If we now factorize the argument of the remaining exponential function as $\boldsymbol{x}^{\mathrm{T}} \boldsymbol{A}^{\mathrm{T}} \boldsymbol{Q}^{-1} \boldsymbol{\psi}=g(\boldsymbol{x})^{\mathrm{T}} f(\boldsymbol{\psi})$ such that $f(\boldsymbol{\psi})$ is of minimal dimension, then $\boldsymbol{t}=f(\boldsymbol{y})$ is a minimal sufficient statistic of the parameter vector $\boldsymbol{x}$. A minimal sufficient statistic of $\boldsymbol{x}$ is therefore given by

$$
\boldsymbol{t}=\boldsymbol{A}^{\mathrm{T}} \boldsymbol{Q}^{-1} \boldsymbol{y}
$$

whose dimension is the number of parameters, i.e., the dimension of $\boldsymbol{x}$, instead of the number of measurements. The matrix $\boldsymbol{A}^{\mathrm{T}} \boldsymbol{Q}^{-1}$ is also referred to as the matched filter in communication 
systems. It is noted that any statistic of the form $\boldsymbol{t}=\boldsymbol{X}^{-1} \boldsymbol{A}^{\mathrm{T}} \boldsymbol{Q}^{-1} \boldsymbol{y}$ is also a minimal sufficient statistic of $\boldsymbol{x}$. This includes in particular the weighted least-squares estimate

$$
\hat{\boldsymbol{x}}=\left(\boldsymbol{A}^{\mathrm{T}} \boldsymbol{Q}^{-1} \boldsymbol{A}\right)^{-1} \boldsymbol{A}^{\mathrm{T}} \boldsymbol{Q}^{-1} \boldsymbol{y}
$$

which is defined as the float solution in Section 2.4. The matrix $\left(\boldsymbol{A}^{\mathrm{T}} \boldsymbol{Q}^{-1} \boldsymbol{A}\right)^{-1} \boldsymbol{A}^{\mathrm{T}} \boldsymbol{Q}^{-1}$ is also called the zero forcing filter in communications.

\section{A3. Free $y^{R}$ Variates}

We consider a system of linear equations that can be partitioned in the form

$$
\left[\begin{array}{c}
\boldsymbol{y} \\
\boldsymbol{y}^{\mathrm{R}}
\end{array}\right]=\left[\begin{array}{cc}
\boldsymbol{A} & \mathbf{0} \\
\mathbf{0} & \boldsymbol{I}_{m}
\end{array}\right] \cdot\left[\begin{array}{c}
\boldsymbol{x} \\
\mathrm{E}\left[\boldsymbol{y}^{\mathrm{R}}\right]
\end{array}\right]+\left[\begin{array}{c}
\boldsymbol{\eta} \\
\boldsymbol{\eta}^{\mathrm{R}}
\end{array}\right], \quad \text { with } \quad\left[\begin{array}{c}
\boldsymbol{\eta} \\
\boldsymbol{\eta}^{\mathrm{R}}
\end{array}\right] \sim \mathcal{G}\left(\left[\begin{array}{l}
\mathbf{0} \\
\mathbf{0}
\end{array}\right],\left[\begin{array}{cc}
\boldsymbol{Q}_{\boldsymbol{y}} & \boldsymbol{Q}_{\boldsymbol{y} \boldsymbol{y}^{\mathrm{R}}} \\
\boldsymbol{Q}_{\boldsymbol{y}^{\mathrm{R}} \boldsymbol{y}} & \boldsymbol{Q}_{\boldsymbol{y}^{\mathrm{R}}}
\end{array}\right]\right) .
$$

The combined measurement vector consists of $\boldsymbol{y} \in \mathbb{R}^{q}$ and $\boldsymbol{y}^{\mathrm{R}} \in \mathbb{R}^{m}$, the combined parameter vector of $\boldsymbol{x} \in \mathbb{R}^{p}$ and $\mathrm{E}\left[\boldsymbol{y}^{\mathrm{R}}\right] \in \mathbb{R}^{m}$, and the combined noise vector of $\boldsymbol{\eta} \in \mathbb{R}^{q}$ and $\boldsymbol{\eta}^{\mathrm{R}} \in \mathbb{R}^{m}$. The system matrix $\boldsymbol{A}$ is of dimension $q \times p$. The entries of $\boldsymbol{y}^{\mathrm{R}}$ in such a system are called free variates (Teunissen 2000). Let the inverse of the noise covariance matrix be partitioned as

$$
\left[\begin{array}{cc}
\boldsymbol{Q}_{\boldsymbol{y}} & \boldsymbol{Q}_{\boldsymbol{y} \boldsymbol{y}^{\mathrm{R}}} \\
\boldsymbol{Q}_{\boldsymbol{y}^{\mathrm{R}} \boldsymbol{y}} & \boldsymbol{Q}_{\boldsymbol{y}^{\mathrm{R}}}^{\mathrm{R}}
\end{array}\right]^{-1}=\left[\begin{array}{cc}
\boldsymbol{G}_{\boldsymbol{y}} & \boldsymbol{G}_{\boldsymbol{y} \boldsymbol{y}^{\mathrm{R}}} \\
\boldsymbol{G}_{\boldsymbol{y}^{\mathrm{R}} \boldsymbol{y}} & \boldsymbol{G}_{\boldsymbol{y}^{\mathrm{R}}}
\end{array}\right]
$$

where all $\boldsymbol{G}_{*}$ are of the same dimension as the corresponding $\boldsymbol{Q}_{*}$.

The normal equations of to the weighted least-squares estimates $\hat{\boldsymbol{x}}$ and $\hat{\boldsymbol{y}}^{\mathrm{R}}$ of the parameter vector are given by

$$
\left[\begin{array}{cc}
\boldsymbol{A}^{\mathrm{T}} & \mathbf{0} \\
\mathbf{0} & \boldsymbol{I}_{m}
\end{array}\right]\left[\begin{array}{cc}
\boldsymbol{G}_{\boldsymbol{y}} & \boldsymbol{G}_{\boldsymbol{y} \boldsymbol{y}^{\mathrm{R}}} \\
\boldsymbol{G}_{\boldsymbol{y}^{\mathrm{R}} \boldsymbol{y}} & \boldsymbol{G}_{\boldsymbol{y}^{\mathrm{R}}}
\end{array}\right]\left[\begin{array}{cc}
\boldsymbol{A} & \mathbf{0} \\
\mathbf{0} & \boldsymbol{I}_{m}
\end{array}\right]\left[\begin{array}{c}
\hat{\boldsymbol{x}} \\
\hat{\boldsymbol{y}}^{\mathrm{R}}
\end{array}\right]=\left[\begin{array}{cc}
\boldsymbol{A}^{\mathrm{T}} & \mathbf{0} \\
\mathbf{0} & \boldsymbol{I}_{m}
\end{array}\right]\left[\begin{array}{cc}
\boldsymbol{G}_{\boldsymbol{y}} & \boldsymbol{G}_{\boldsymbol{y} \boldsymbol{y}^{\mathrm{R}}} \\
\boldsymbol{G}_{\boldsymbol{y}^{\mathrm{R}} \boldsymbol{y}} & \boldsymbol{G}_{\boldsymbol{y}^{\mathrm{R}}}
\end{array}\right]\left[\begin{array}{c}
\boldsymbol{y} \\
\boldsymbol{y}^{\mathrm{R}}
\end{array}\right] .
$$

From the normal equations we get the following two conditions

$$
\begin{aligned}
& \boldsymbol{A}^{\mathrm{T}} \boldsymbol{G}_{\boldsymbol{y}} \boldsymbol{A} \hat{\boldsymbol{x}}+\boldsymbol{A}^{\mathrm{T}} \boldsymbol{G}_{\boldsymbol{y} \boldsymbol{y}^{\mathrm{R}}} \hat{\boldsymbol{y}}^{\mathrm{R}}=\boldsymbol{A}^{\mathrm{T}} \boldsymbol{G}_{\boldsymbol{y}} \boldsymbol{y}+\boldsymbol{A}^{\mathrm{T}} \boldsymbol{G}_{\boldsymbol{y} \boldsymbol{y}^{\mathrm{R}}} \boldsymbol{y}^{\mathrm{R}} \\
& \boldsymbol{G}_{\boldsymbol{y}^{\mathrm{R}} \boldsymbol{y}} \boldsymbol{A} \hat{\boldsymbol{x}}+\boldsymbol{G}_{\boldsymbol{y}^{\mathrm{R}}} \hat{\boldsymbol{y}}^{\mathrm{R}}=\boldsymbol{G}_{\boldsymbol{y}^{\mathrm{R}} \boldsymbol{y}} \boldsymbol{y}+\boldsymbol{G}_{\boldsymbol{y}^{\mathrm{R}}} \boldsymbol{y}^{\mathrm{R}} .
\end{aligned}
$$

Condition (A21) can be reformulated as $\hat{\boldsymbol{y}}^{\mathrm{R}}=\boldsymbol{G}_{\boldsymbol{y}^{\mathrm{R}}}^{-1}\left(\boldsymbol{G}_{\boldsymbol{y}^{\mathrm{R}} \boldsymbol{y}} \boldsymbol{y}+\boldsymbol{G}_{\boldsymbol{y}^{\mathrm{R}}} \boldsymbol{y}^{\mathrm{R}}-\boldsymbol{G}_{\boldsymbol{y}^{\mathrm{R}} \boldsymbol{y}} \boldsymbol{A} \hat{\boldsymbol{x}}\right)$. Substituting this expression into condition (A20) leads to

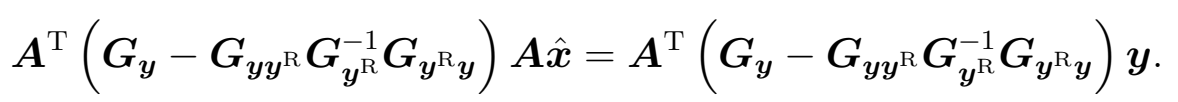

This equation defines the estimates $\hat{\boldsymbol{x}}$, which we now want to express in terms of the covariance matrices of the noise vectors $\boldsymbol{\eta}$ and $\boldsymbol{\eta}^{\mathrm{R}}$. From (A18) we know that

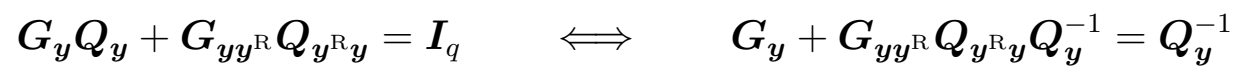

$$
\begin{aligned}
& \boldsymbol{G}_{\boldsymbol{y}^{\mathrm{R}} \boldsymbol{y}} \boldsymbol{Q}_{\boldsymbol{y}}+\boldsymbol{G}_{\boldsymbol{y}^{\mathrm{R}}} \boldsymbol{Q}_{\boldsymbol{y}^{\mathrm{R}} \boldsymbol{y}}=\mathbf{0} \quad \Longleftrightarrow \quad-\boldsymbol{G}_{\boldsymbol{y}^{\mathrm{R}}}^{-1} \boldsymbol{G}_{\boldsymbol{y}^{\mathrm{R}} \boldsymbol{y}}=\boldsymbol{Q}_{\boldsymbol{y}^{\mathrm{R}} \boldsymbol{y}} \boldsymbol{Q}_{\boldsymbol{y}}^{-1}
\end{aligned}
$$

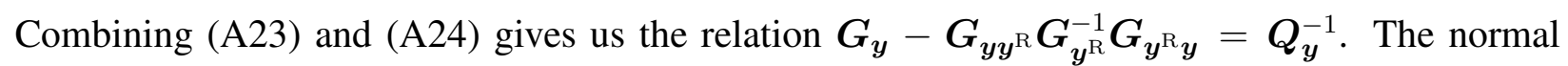
equation (A22) defining the weighted least-squares estimate $\hat{\boldsymbol{x}}$ can thus be rewritten as

$$
\boldsymbol{A}^{\mathrm{T}} \boldsymbol{Q}_{\boldsymbol{y}}^{-1} \boldsymbol{A} \hat{\boldsymbol{x}}=\boldsymbol{A}^{\mathrm{T}} \boldsymbol{Q}_{\boldsymbol{y}}^{-1} \boldsymbol{y}
$$


That is, the estimate $\hat{\boldsymbol{x}}$ is exactly identical to the one that would result from the reduced system of equations $\boldsymbol{y}=\boldsymbol{A} \boldsymbol{x}+\boldsymbol{\eta}$, with $\boldsymbol{\eta} \sim \mathcal{G}\left(\mathbf{0}, \boldsymbol{Q}_{\boldsymbol{y}}\right)$. Consequently, if one is not interested in the estimates $\hat{\boldsymbol{y}}^{\mathrm{R}}$, the measurements $\boldsymbol{y}^{\mathrm{R}}$ and the parameter vector $\mathrm{E}\left[\boldsymbol{y}^{\mathrm{R}}\right]$ can simply be removed from the system of equations (A17).

It is noted that the estimates $\hat{\boldsymbol{y}}^{\mathrm{R}}$ very well depend on the part of the system of equations (A17) that corresponds to the observations $\boldsymbol{y}$ and cannot be computed independently. From (A21) and (A24) these estimates follow as

$$
\hat{\boldsymbol{y}}^{\mathrm{R}}=\boldsymbol{y}^{\mathrm{R}}-\boldsymbol{Q}_{\boldsymbol{y}^{\mathrm{R}} \boldsymbol{y}} \boldsymbol{Q}_{\boldsymbol{y}}^{-1}(\boldsymbol{y}-\boldsymbol{A} \hat{\boldsymbol{x}}) .
$$

\section{A4. Constrained Optimization}

An optimization problem has the form

$$
\boldsymbol{x}^{*}=\underset{\boldsymbol{x} \in \mathbb{X}}{\operatorname{argmin}} f(\boldsymbol{x}),
$$

with the optimization variable $\boldsymbol{x} \in \mathbb{R}^{p}$, the objective function $f(\cdot): \mathbb{R}^{p} \mapsto \mathbb{R}$, and the feasible region $\mathbb{X} \subseteq \mathbb{R}^{p}$. The vector $\boldsymbol{x}^{*}$ is the solution of the problem, if it leads to the smallest value of the objective function among all vectors in $\mathbb{X}$. In the following, the nature of $\mathbb{X}$ is specified explicitly in terms of a set of inequalities and equalities as

$$
\begin{aligned}
\mathbb{X}=\left\{\boldsymbol{x} \in \mathbb{R}^{p} \mid g^{k}(\boldsymbol{x})\right. & \leq 0, k=1, \ldots, K \\
h^{j}(\boldsymbol{x}) & =0, j=1, \ldots, J\},
\end{aligned}
$$

with the inequality constraint functions $g^{k}(\cdot): \mathbb{R}^{p} \mapsto \mathbb{R}$ and the equality constraint functions $h^{k}(\cdot): \mathbb{R}^{p} \mapsto \mathbb{R}$. Most optimization problems are very difficult to solve, meaning that determining $\boldsymbol{x}^{*}$ can imply a very long computation time or is practically not feasible at all. One exception is the class of convex optimization problems, for which the objective and inequality constraint functions are convex and the equality constraint functions are affine. The so defined feasible region $\mathbb{X}$ is convex, so that a convex optimization problem is identical to minimizing a convex function over a convex set. Although convex optimization problems can generally not be solved in closed form, there are efficient and reliable methods to find $\boldsymbol{x}^{*}$.

Within this dissertation, only a few specific aspects of how to solve optimization problems are discussed. More information can be found in textbooks like Boyd and Vandenberghe (2004) or Bazaraa et al. (2013). A prominent example of optimality conditions are the Karush-Kuhn-Tucker (KKT) conditions. A point $\boldsymbol{x}^{*}$ is said to be a KKT point, if the following three conditions are met:

$$
\begin{aligned}
\text { PF: } & g^{k}\left(\boldsymbol{x}^{*}\right) \leq 0, k=1, \ldots, K \\
& h^{j}\left(\boldsymbol{x}^{*}\right)=0, j=1, \ldots, J \\
\text { DF: } & \nabla f\left(\boldsymbol{x}^{*}\right)+\sum_{k=1}^{K} u^{k} \nabla g^{k}\left(\boldsymbol{x}^{*}\right)+\sum_{j=1}^{J} v^{j} \nabla h^{j}\left(\boldsymbol{x}^{*}\right)=\mathbf{0}, \quad \text { with } u^{k} \geq 0, k=1, \ldots, K \\
\mathrm{CS}: & u^{k} g^{k}\left(\boldsymbol{x}^{*}\right)=0, \quad k=1, \ldots, K .
\end{aligned}
$$

The primal feasibility (PF) condition states that $\boldsymbol{x}^{*}$ is within the feasible region $\mathbb{X}$, the dual feasibility (DF) condition results from Gordon's theorem, and the complementary slackness (CS) condition ensures that DF exclusively includes gradients of active constraints with $g^{k}\left(\boldsymbol{x}^{*}\right)=0$. The parameters $u^{k} \in \mathbb{R}, u^{k} \geq 0$, and $v^{j} \in \mathbb{R}$ are called Lagrangian multipliers. 
Suppose that the objective function $f(\cdot)$, the constraint functions $g^{k}(\cdot)$ of all active inequality constraints, and the constraint functions $h^{j}(\cdot)$ are differentiable at a KKT point $\boldsymbol{x}^{*}$, then $\boldsymbol{x}^{*}$ is a local minimizer, i.e., the KKT conditions are sufficient conditions for a local optimum. Given that the optimization problem is convex, a KKT point $\boldsymbol{x}^{*}$ is a global minimizer.

The KKT conditions are necessary conditions for $\boldsymbol{x}^{*}$ to be a local minimizer, if certain constraint qualifications are fulfilled, so that the duality gap is zero. These constraint qualifications guarantee that by only examining KKT points, we do not miss a local minimizer and, thus, a possibly global minimizer. One version of the constraint qualifications is that the gradients of all active constraints are linear independent at the KKT point $\boldsymbol{x}^{*}$. Given a convex optimization problem, we can make use Slater's constraint qualifications, which state that the interior of $\mathbb{X}$ is non-empty, meaning that $\exists \boldsymbol{x}: g^{k}(\boldsymbol{x})<0, k=1, \ldots, K, h^{j}(\boldsymbol{x})=0, j=1, \ldots, J$. Slater's condition implies the regularity of all feasible points, not only of one specific KKT point.

Accordingly, if some constraint qualifications are met and the optimization problem (A27) is convex, it can be solved by finding a KKT point. There are a few special cases in which the KKT conditions can be solved directly. A prominent example of such a solution is the water-filling algorithm, used for instance to allocate transmit power to a set of communication channels. Many dual techniques such as the subgradient or cutting plane method can be interpreted as a way to numerically solve the KKT system. If the optimization problem is not convex, but the constraint qualifications are met, the solution of (A27) can be found by examining all KKT points, which is generally still a very difficult problem. One approach that is often followed is to hope that by proper initialization an optimization algorithm will converge to the KKT point that corresponds to the global minimum, although this cannot be guaranteed. Also, a non-convex optimization problem might still have some useful properties such as monotonic objective functions or normal feasible sets that can be helpful in order to solve the problem. 


\section{Bibliography}

Agrell E, Eriksson T, Vardy A, Zeger K (2002) Closest Point Search in Lattices. IEEE Transactions on Information Theory 48(8):2201-2214

Amiri-Simkooei AR (2007) Least-squares variance component estimation: theory and GPS applications. Dissertation, TU Delft, Delft University of Technology

Baarda W (1968) A testing procedure for use in geodetic networks. Netherlands Geodetic Commission, Publications on Geodesy, New Series 2(5)

Baarda W (1973) S-transformations and criterion matrices. Netherlands Geodetic Commission, Publications on Geodesy, New Series 5(1)

Babai L (1986) On Lovasz' Lattice Reduction and the Nearest Lattice Point Problem. Combinatorica 6(1):1-13

Bazaraa MS, Sherali HD, Shetty CM (2013) Nonlinear Programming: Theory and Algorithms. John Wiley \& Sons

BDS-SIS-ICD (2016) BeiDou navigation satellite system signal in space interface control document. Version 2.1

Bertiger W, Desai SD, Haines B, Harvey N, Moore AW, Owen S, Weiss JP (2010) Single receiver phase ambiguity resolution with GPS data. Journal of Geodesy 84(5):327-337

Betti B, Crespi M, Sanso F (1993) A geometric illustration of ambiguity resolution in GPS theory and a Bayesian approach. Manuscripta Geodaetica 18:317-330

Bisnath S, Gao Y (2008) Current State of Precise Point Positioning and Future Prospects and Limitations. In: Proceedings of IAG Symposia no 133, Observing our Changing Earth, Perugia, Italy, pp 615-623

Bisnath S, Petovello M, Lachapelle G (2007) What is carrier phase wind-up? What is its effect on GNSS performance/operation? InsideGNSS July/August 2007:32-35

Blewitt G (1989) Carrier phase ambiguity resolution for the Global Positioning System applied to geodetic baselines up to $2000 \mathrm{~km}$. Journal of Geophysical Research: Solid Earth 94(B8):10,187-10,203

Bock Y (1998) Medium distance GPS measurements. In: GPS for Geodesy, Springer, pp 483-536

Boehm J, Niell A, Tregoning P, Schuh H (2006a) Global Mapping Function (GMF): A new empirical mapping function based on numerical weather model data. Geophysical Research Letters $33(7)$ 
Boehm J, Werl B, Schuh H (2006b) Troposphere mapping functions for GPS and very long baseline interferometry from European Centre for Medium-Range Weather Forecasts operational analysis data. Journal of Geophysical Research: Solid Earth 111(B2)

Borre K, Tiberius CCJM (2000) Time series analysis of GPS observables. In: Proceedings of ION GPS 2000, Salt Lake City, UT, USA, pp 1885-1894

Boyd S, Vandenberghe L (2004) Convex optimization. Cambridge university press

Brack A (2015) On reliable data-driven partial GNSS ambiguity resolution. GPS Solutions 19(3):411-422

Brack A (2016a) Optimal estimation of a subset of integers with application to GNSS. Artificial Satellites 51(4):123-134

Brack A (2016b) Partial Ambiguity Resolution for Reliable GNSS Positioning - a Useful Tool? In: Proceedings of IEEE Aerospace Conference 2016, IEEE, Big Sky, MT, USA, pp 1-7

Brack A (2017a) Long Baseline GPS+BDS RTK Positioning with Partial Ambiguity Resolution. In: Proceedings of ION ITM 2017, Monterey, CA, USA, pp 754-762

Brack A (2017b) Reliable GPS+BDS RTK positioning with partial ambiguity resolution. GPS Solutions 21(3):1083-1092

Brack A, Günther C (2014) Generalized integer aperture estimation for partial GNSS ambiguity fixing. Journal of Geodesy 88(5):479-490

Brack A, Henkel P, Günther C (2013) Sequential Best Integer-Equivariant Estimation for Geodetic Network Solutions. In: Proceedings of ION ITM 2013, San Diego, CA, USA, pp 310-317

Brack A, Henkel P, Günther C (2014) Sequential Best Integer-Equivariant Estimation for GNSS. Navigation 61(2):149-158

Brown RG, Hwang PYC (1983a) A Kalman filter approach to precision GPS geodesy. Navigation 30(4):338-349

Brown RG, Hwang PYC (1983b) Application of GPS to geodesy: A combination problem in estimation and large-scale multiple hypothesis testing. In: Proceedings of IEEE NTC 1983, San Francisco, CA, USA, pp 104-111

Brown RG, Hwang PYC (1986) GPS failure detection by autonomous means within the cockpit. Navigation 33(4):335-353

Cao W, O'Keefe K, Cannon M (2007) Partial ambiguity fixing within multiple frequencies and systems. In: Proceedings of ION GNSS 2007, Forth Worth, TX, USA, pp 312-323

Cassels JWS (1971) An introduction to the geometry of numbers. Springer Science \& Business Media

Chang XW, Yang X, Zhou T (2005) MLAMBDA: a modified LAMBDA method for integer leastsquares estimation. Journal of Geodesy 79(9):552-565 
Cocard M, Geiger A (1992) Systematic search for all possible widelanes. In: Proceedings of 6th International Geodetic Symposium on Satellite Positioning, Columbus, OH, USA, pp 17-20

Cocard M, Bourgon S, Kamali O, Collins P (2008) A systematic investigation of optimal carrierphase combinations for modernized triple-frequency GPS. Journal of Geodesy 82(9):555-564

Collins P (2008) Isolating and estimating undifferenced GPS integer ambiguities. In: Proceedings of ION NTM 2008, San Diego, CA, USA, pp 720-732

Deng C, Tang W, Liu J, Shi C (2014) Reliable single-epoch ambiguity resolution for short baselines using combined GPS/BeiDou system. GPS Solutions 18(3):375-386

Euler HJ, Goad CC (1991) On optimal filtering of GPS dual frequency observations without using orbit information. Bulletin Geodesique 65(2):130-143

Euler HJ, Schaffrin B (1991) On a Measure for the Discernibility Between Different Ambiguity Solutions in the Static-Kinematic GPS-Mode. In: Proceedings of IAG Symposia no 107, Kinematic Systems in Geodesy, Surveying, and Remote Sensing, Banff, Canada, pp 285-295

Feng Y, Wang J (2011) Computed success rates of various carrier phase integer estimation solutions and their comparison with statistical success rates. Journal of Geodesy 85(2):93-103

Fincke U, Pohst M (1985) Improved methods for calculating vectors of short length in a lattice, including a complexity analysis. Mathematics of Computation 44(170):463-471

Frei E, Beutler G (1990) Rapid Static Positioning Based on the Fast Ambiguity Resolution Approach FARA: Theory and First Results. Manuscripta Geodaetica 15(6):325-356

Ge M, Gendt G, Rothacher M, Shi C, Liu J (2008) Resolution of GPS carrier-phase ambiguities in precise point positioning (PPP) with daily observations. Journal of Geodesy 82(7):389-399

Geng J, Shi C, Ge M, Dodson AH, Lou Y, Zhao Q, Liu J (2012) Improving the estimation of fractional-cycle biases for ambiguity resolution in precise point positioning. Journal of Geodesy 86(8):579-589

GLONASS-ICD (2008) GLONASS interface control document, navigational radiosignals in bands L1, L2. Version 5.1

Gruber PM, Lekkerkerker CG (1987) Geometry of numbers. North-Holland

Gundlich B, Koch KR (2002) Confidence regions for GPS baselines by Bayesian statistics. Journal of Geodesy 76(1):55-62

Günther C, Henkel P (2012) Integer Ambiguity Estimation for Satellite Navigation. IEEE Transactions on Signal Processing 60(7):3387-3393

Hagenauer J, Offer E, Papke L (1996) Iterative Decoding of Binary Block and Convolutional Codes. IEEE Transactions on Information Theory 42(2):429-445

Han S (1997) Quality-control issues relating to instantaneous ambiguity resolution for real-time GPS kinematic positioning. Journal of Geodesy 71(6):351-361 
Han S, Rizos C (1996) Integrated method for instantaneous ambiguity resolution using new generation GPS receivers. In: Proceedings of IEEE PLANS 1996, Atlanta, GA, USA, pp 254-261

Harville DA (1977) Maximum likelihood approaches to variance component estimation and to related problems. Journal of the American Statistical Association 72(358):320-338

Hassibi A, Boyd S (1998) Integer Parameter Estimation in Linear Models with Applications to GPS. IEEE Transactions on Signal Processing 46(11):2938-2952

Hatch RR (1982) The synergism of GPS code and carrier measurements. In: Proceedings of 3rd International Symposium on Satellite Doppler Positioning, Las Cruces, NM, USA, pp 12131231

He H, Li J, Yang Y, Xu J, Guo H, Wang A (2014) Performance assessment of single- and dualfrequency BeiDou/GPS single-epoch kinematic positioning. GPS Solutions 18(3):393-403

Hegarty C, Powers E, Fonville B (2005) Accounting for Timing Biases between GPS, Modernized GPS, and Galileo Signals. In: Proceedings of ION PTTI 2004, Washington, DC, USA, pp 307318

Henkel P (2015) Tightly coupled precise point positioning and attitude determination. IEEE Transactions on Aerospace and Electronic Systems 51(4):3182-3197

Henkel P, Günther C (2007) Three frequency linear combinations for Galileo. In: Proceedings of 4th Workshop on Positioning, Navigation and Communication (IEEE WPNC 2007), Hannover, Germany, pp 239-245

Hochwald BM, Ten Brink S (2003) Achieving near-capacity on a multiple-antenna channel. IEEE Transactions on Communications 51(3):389-399

Hou Y, Verhagen S, Wu J (2016) An Efficient Implementation of Fixed Failure-Rate Ratio Test for GNSS Ambiguity Resolution. Sensors 16(7):945

Imparato D (2016) GNSS-based receiver autonomous integrity monitoring for aircraft navigation. Dissertation, TU Delft, Delft University of Technology

IS-GPS-200 (2016) GPS space segment/navigation user segment interface for radio frequency link 1 (L1) and link 2 (L2). Revision H, IRN003

IS-GPS-705 (2014) GPS space segment/navigation user segment interface for radio frequency link 5 (L5). Revision D, IRN005

IS-GPS-800 (2014) GPS space segment/navigation user segment interface for radio frequency link 1 (L1), signal L1 civil (L1C). Revision D, IRN004

IS-QZSS (2016) Quasi-zenith satellite system navigation service interface specification. Version 1.8

ISRO-IRNSS-ICD-SPS (2014) Indian regional navigation satellite system signal in space ICD for standard positioning service. Version 1.0 
Jaldén J, Ottersten B (2005) Parallel Implementation of a Soft Output Sphere Decoder. In: Conference Record of the Thirty-Ninth Asilomar Conference on Signals, Systems and Computers, 2005, IEEE, Pacific Grove, CA, USA, pp 581-585

Jazaeri S, Amiri-Simkooei AR, Sharifi MA (2012) Fast integer least-squares estimation for GNSS high-dimensional ambiguity resolution using lattice theory. Journal of Geodesy 86(2):123-136

Jazaeri S, Amiri-Simkooei AR, Sharifi MA (2014) On lattice reduction algorithms for solving weighted integer least squares problems: comparative study. GPS Solutions 18(1):105-114

de Jonge PJ (1998) A processing strategy for the application of the GPS in networks. Dissertation, TU Delft, Delft University of Technology

de Jonge PJ, Tiberius CCJM (1996) The LAMBDA method for integer ambiguity estimation: implementation aspects. Publications of the Delft Computing Centre, LGR-Series 12

Kay SM (1993) Fundamentals of Statistical Signal Processing: Estimation Theory. Prentice Hall

Khanafseh S, Pervan B (2010) New Approach for Calculating Position Domain Integrity Risk for Cycle Resolution in Carrier Phase Navigation Systems. IEEE Transactions on Aerospace and Electronic Systems 46(1):296-307

Khodabandeh A, Teunissen PJG (2014) Array-based satellite phase bias sensing: theory and GPS/BeiDou/QZSS results. Measurement Science and Technology 25(9):095,801

Khodabandeh A, Teunissen PJG (2015) An analytical study of PPP-RTK corrections: precision, correlation and user-impact. Journal of Geodesy 89(11):1109-1132

Kleijer F (2004) Troposphere modeling and filtering for precise GPS leveling. Dissertation, TU Delft, Delft University of Technology

Kouba J, Héroux P (2001) Precise point positioning using IGS orbit and clock products. GPS Solutions 5(2):12-28

Laurichesse D, Mercier F (2007) Integer ambiguity resolution on undifferenced GPS phase measurements and its application to PPP. In: Proceedings of ION GNSS 2007, Fort Worth, TX, USA, pp 839-848

Laurichesse D, Mercier F, Berthias JP, Broca P, Cerri L (2009) Integer ambiguity resolution on undifferenced GPS phase measurements and its application to PPP and satellite precise orbit determination. Navigation 56(2):135-149

Lee YC (1986) Analysis of range and position comparison methods as a means to provide GPS integrity in the user receiver. In: Proceedings of the 42nd Annual Meeting of the Institute of Navigation, Seattle, WA, pp 1-4

Leick A, Rapoport L, Tatarnikov D (2015) GPS satellite surveying. John Wiley \& Sons

Lenstra AK, Lenstra HW, Lovasz L (1982) Factoring Polynomials with Rational Coefficients. Mathematische Annalen 261(4):515-534 
Magill DT (1965) Optimal Adaptive Estimation of Sampled Stochastic Processes. IEEE Transactions on Automatic Control 10(4):434-439

Makarov VL, Rubinov AM (1977) Mathematical Theory of Economic Dynamics and Equilibria. Springer Verlag

Mervart L, Lukes Z, Rocken C, Iwabuchi T (2008) Precise Point Positioning with ambiguity resolution in real-time. In: Proceedings of ION GNSS 2008, Savannah, GA, USA, pp 397-405

Montenbruck O, Hauschild A, Hessels U (2011) Characterization of GPS/GIOVE sensor stations in the CONGO network. GPS Solutions 15(3):193-205

MOPS (1999) Minimum operational performance standards for global positioning system/wide area augmentation system airborne equipment. RTCA Inc Document No RTCA/DO-229B

Mowlam A (2004) Baseline precision results using triple frequency partial ambiguity sets. In: Proceedings of ION GNSS 2004, Long Beach, CA, USA, pp 2509-2518

Nardo A, Li B, Teunissen PJG (2016) Partial Ambiguity Resolution for Ground and Space-Based Applications in a GPS + Galileo Scenario: A Simulation Study. Advances in Space Research 57(1):30-45

Niell AE (1996) Global mapping functions for the atmosphere delay at radio wavelengths. Journal of Geophysical Research: Solid Earth 101(B2):3227-3246

Odijk D (2000) Weighting ionospheric corrections to improve fast GPS positioning over medium distances. In: Proceedings of ION GPS 2000, Salt Lake City, UT, USA, pp 1113-1123

Odijk D (2002) Fast precise GPS positioning in the presence of ionospheric delays. Dissertation, TU Delft, Delft University of Technology

Odijk D, Teunissen PJG (2013a) Characterization of between-receiver GPS-Galileo inter-system biases and their effect on mixed ambiguity resolution. GPS Solutions 17(4):521-533

Odijk D, Teunissen PJG (2013b) Estimation of differential inter-system biases between the overlapping frequencies of GPS, Galileo, BeiDou and QZSS. In: Proceedings of 4th International Colloquium on Scientific and Fundamental Aspects of the Galileo Programme, Prague, Czech Republic

Odijk D, Teunissen PJG, Zhang B (2012) Single-frequency integer ambiguity resolution enabled GPS precise point positioning. Journal of Surveying Engineering 138(4):193-202

Odijk D, Arora BS, Teunissen PJG (2014a) Predicting the Success Rate of Long-baseline GPS+Galileo (Partial) Ambiguity Resolution. Journal of Navigation 67(3):385-401

Odijk D, Teunissen PJG, Khodabandeh A (2014b) Single-frequency PPP-RTK: theory and experimental results. In: Earth on the Edge: Science for a Sustainable Planet, Springer, pp 571-578

Odijk D, Zhang B, Khodabandeh A, Odolinski R, Teunissen PJG (2016) On the estimability of parameters in undifferenced, uncombined GNSS network and PPP-RTK user models by means of $\mathcal{S}$-system theory. Journal of Geodesy 90(1):15-44 
Odijk D, Nadarajah N, Zaminpardaz S, Teunissen PJG (2017) GPS, Galileo, QZSS and IRNSS differential ISBs: estimation and application. GPS Solutions 21(2):439-450

Odolinski R, Teunissen PJG (2016) Single-frequency, dual-GNSS versus dual-frequency, singleGNSS: a low-cost and high-grade receivers GPS-BDS RTK analysis. Journal of Geodesy 90(11):1255-1278

Odolinski R, Teunissen PJG, Odijk D (2014a) Combined GPS+BDS+Galileo+QZSS for Long Baseline RTK Positioning. In: Proceedings of ION GNSS+ 2014, Tampa, FL, USA, pp 23262340

Odolinski R, Teunissen PJG, Odijk D (2014b) First combined COMPASS/BeiDou-2 and GPS positioning results in Australia. Part II: Single-and multiple-frequency single-baseline RTK positioning. Journal of Spatial Science 59(1):25-46

Odolinski R, Teunissen PJG, Odijk D (2015a) Combined BDS, Galileo, QZSS and GPS singlefrequency RTK. GPS Solutions 19(1):151-163

Odolinski R, Teunissen PJG, Odijk D (2015b) Combined GPS + BDS for short to long baseline RTK positioning. Measurement Science and Technology 26(4):45,801

Oleynik E (2012) GLONASS Status and Modernization. Presented at United Nations/Latvia Workshop on the Applications of Global Navigation Satellite Systems, Riga, Latvia

OS-SIS-ICD (2016) Galileo open service signal-in-space interface control document. Version 1.3

Parkins A (2011) Increasing GNSS RTK availability with a new single-epoch batch partial ambiguity resolution algorithm. GPS Solutions 15(4):391-402

Parkinson BW, Axelrad P (1988) Autonomous GPS integrity monitoring using the pseudorange residual. Navigation 35(2):255-274

Pesyna KM, Heath RW, Humphreys TE (2014) Centimeter positioning with a smartphone-quality GNSS antenna. In: Proceedings of ION GNSS+ 2014, Tampa, FL, USA, pp 1568-1577

Poor HV (1994) An Introduction to Signal Detection and Estimation. Springer

Psiaki ML, Mohiuddin S (2007) Modeling, analysis, and simulation of GPS carrier phase for spacecraft relative navigation. Journal of Guidance, Control, and Dynamics 30(6):1628-1639

Robertson P, Villebrun E, Hoeher P (1995) A comparison of optimal and sub-optimal MAP decoding algorithms operating in the log domain. In: Proceedings of IEEE ICC 1995, Gateway to Globalization, IEEE, Seattle, WA, USA, vol 2, pp 1009-1013

Saastamoinen J (1972) Atmospheric correction for the troposphere and stratosphere in radio ranging satellites. The Use of Artificial Satellites for Geodesy pp 247-251

Schaffrin B, Bock Y (1988) A unified scheme for processing GPS dual-band phase observations. Bulletin Geodesique 62(2):142-160

Schnorr CP, Euchner M (1994) Lattice basis reduction: improved practical algorithms and solving subset sum problems. Mathematical Programming 66(1-3):181-199 
Seysen M (1993) Simultaneous reduction of a lattice basis and its reciprocal basis. Combinatorica 13(3):363-376

Sims F, Lainiotis D, Magill D (1969) Recursive Algorithm for the Calculation of the Adaptive Kalman Filter Weighting Coefficients. IEEE Transactions on Automatic Control 14(2):215-218

Steingrimsson B, Luo ZQ, Wong KM (2003) Soft quasi-maximum-likelihood detection for multiple-antenna wireless channels. IEEE Transactions on Signal Processing 51(11):2710-2719

Studer C, Bölcskei H (2010) Soft-Input Soft-Output Single Tree-Search Sphere Decoding. IEEE Transactions on Information Theory 56(10):4827-4842

Studer C, Burg A, Bölcskei H (2008) Soft-Output Sphere Decoding: Algorithms and VLSI Implementation. IEEE Journal on Selected Areas in Communications 26(2):290-300

Sturza MA (1988) Navigation system integrity monitoring using redundant measurements. Navigation 35(4):483-501

Teunissen PJG (1984) Generalized inverses, adjustment, the datum problem and S-transformations. Lecture notes International School of Geodesy, Erice, Italy, April 25-May 10

Teunissen PJG (1990) Quality Control in Integrated Navigation Systems. In: Proceedings of IEEE PLANS 1990, IEEE, Las Vegas, NV, USA, pp 158-165

Teunissen PJG (1993) Least-squares estimation of the integer GPS ambiguities. In: Invited lecture, section IV theory and methodology, IAG general meeting, Beijing, China

Teunissen PJG (1995a) The invertible GPS ambiguity transformations. Manuscripta Geodaetica 20(6):489-497

Teunissen PJG (1995b) The least-squares ambiguity decorrelation adjustment: a method for fast GPS integer ambiguity estimation. Journal of Geodesy 70(1-2):65-82

Teunissen PJG (1997a) A canonical theory for short GPS baselines. Part I: the baseline precision. Journal of Geodesy 71(6):320-336

Teunissen PJG (1997b) A canonical theory for short GPS baselines. Part II: the ambiguity precision and correlation. Journal of Geodesy 71(7):389-401

Teunissen PJG (1997c) A canonical theory for short GPS baselines. Part IV: precision versus reliability. Journal of Geodesy 71(9):513-525

Teunissen PJG (1997d) On the GPS widelane and its decorrelating property. Journal of Geodesy 71(9):577-587

Teunissen PJG (1998a) Quality control and GPS. In: GPS for Geodesy, Springer, pp 271-318

Teunissen PJG (1998b) Success probability of integer GPS ambiguity rounding and bootstrapping. Journal of Geodesy 72(10):606-612

Teunissen PJG (1998c) The ionosphere-weighted GPS baseline precision in canonical form. Journal of Geodesy 72(2):107-111 
Teunissen PJG (1999a) An optimality property of the integer least-squares estimator. Journal of Geodesy 73(11):587-593

Teunissen PJG (1999b) The probability distribution of the GPS baseline for a class of integer ambiguity estimators. Journal of Geodesy 73(5):275-284

Teunissen PJG (2000) Adjustment theory, an introduction. Delft Academic Press, Series on Mathematical Geodesy and Positioning

Teunissen PJG (2002) The parameter distributions of the integer GPS model. Journal of Geodesy 76(1):41-48

Teunissen PJG (2003a) A carrier phase ambiguity estimator with easy-to-evaluate fail rate. Artificial Satellites 38(3):89-96

Teunissen PJG (2003b) Integer aperture GNSS ambiguity resolution. Artificial Satellites 38(3):79_ 88

Teunissen PJG (2003c) Theory of integer equivariant estimation with application to GNSS. Journal of Geodesy 77(7-8):402-410

Teunissen PJG (2003d) Towards a unified theory of GNSS ambiguity resolution. Journal of Global Positioning Systems 2(1):1-12

Teunissen PJG (2004) Penalized GNSS ambiguity resolution. Journal of Geodesy 78(4-5):235-244

Teunissen PJG (2005a) GNSS ambiguity resolution with optimally controlled failure-rate. Artificial Satellites 40(4):219-227

Teunissen PJG (2005b) Integer aperture bootstrapping: a new GNSS ambiguity estimator with controllable fail-rate. Journal of Geodesy 79(6-7):389-397

Teunissen PJG (2005c) Integer aperture least-squares estimation. Artificial Satellites 40:149-160

Teunissen PJG (2005d) On the computation of the best integer equivariant estimator. Artificial Satellites 40(3):161-171

Teunissen PJG, Khodabandeh A (2015) Review and principles of PPP-RTK methods. Journal of Geodesy 89(3):217-240

Teunissen PJG, Kleusberg A (2012) GPS for Geodesy. Springer Science \& Business Media

Teunissen PJG, Verhagen S (2008) GNSS Ambiguity Resolution: When and How to Fix or not to Fix? In: Proceedings of VI Hotine-Marussi Symposium on Theoretical and Computational Geodesy, Springer Berlin Heidelberg, Wuhan, China, pp 143-148

Teunissen PJG, Verhagen S (2009) The GNSS ambiguity ratio-test revisited: a better way of using it. Survey Review 41(312):138-151

Teunissen PJG, Joosten P, Tiberius CCJM (1999) Geometry-free ambiguity success rates in case of partial fixing. In: Proceedings of ION NTM 1999, San Diego, CA, USA, pp 201-207 
Teunissen PJG, Odijk D, Zhang B (2010) PPP-RTK: results of CORS network-based PPP with integer ambiguity resolution. Journal of Aeronautics, Astronautics and Aviation, Series A 42(4):223-230

Teunissen PJG, Odolinski R, Odijk D (2014) Instantaneous BeiDou+GPS RTK positioning with high cut-off elevation angles. Journal of Geodesy 88(4):335-350

Tiberius CCJM (1998) Recursive data processing for kinematic GPS surveying. Dissertation, TU Delft, Delft University of Technology

Tiberius CCJM, de Jonge PJ (1995) Fast Positioning Using the LAMBDA Method. In: Proceedings of DSNS-95, Bergen, Norway

Tiberius CCJM, Pany T, Eissfeller B, Joosten P, Verhagen S (2002) 0.99999999 confidence ambiguity resolution with GPS and Galileo. GPS Solutions 6(1-2):96-99

Tuy H (1999) Normal sets, polyblocks and monotonic optimization. Vietnam Journal of Mathematics 27(4):277-300

Tuy H (2000) Monotonic optimization: Problems and solution approaches. SIAM Journal on Optimization 11(2):464-494

Urlichich Y, Subbotin V, Stupak G, Dvorkin V, Povalyaev A, Karutin S (2011) GLONASS Modernization. In: Proceedings of ION GNSS 2011, Portland, OR, USA, pp 3125-3128

Verhagen S (2003) On the approximation of the integer least-squares success rate: which lower or upper bound to use? Journal of Global Positioning Systems 2(2):117-124

Verhagen S (2005) The GNSS integer ambiguities: estimation and validation. Dissertation, TU Delft, Delft University of Technology

Verhagen S, Teunissen PJG (2005) Performance comparison of the BIE estimator with the float and fixed GNSS ambiguity estimators. In: Proceedings of IAG General Assembly 2003, A Window on the Future of Geodesy, Springer, Sapporo, Japan, pp 428-433

Verhagen S, Teunissen PJG (2006) New Global Navigation Satellite System Ambiguity Resolution Method Compared to Existing Approaches. Journal of Guidance, Control, and Dynamics 29(4):981-991

Verhagen S, Teunissen PJG (2013) The ratio test for future GNSS ambiguity resolution. GPS Solutions 17(4):535-548

Verhagen S, Teunissen PJG, van der Marel H, Li B (2011) GNSS ambiguity resolution: which subset to fix. In: Proceedings of IGNSS Symposium 2011, Sydney, Australia

Verhagen S, Li B, Teunissen PJG (2013) Ps-LAMBDA: Ambiguity success rate evaluation software for interferometric applications. Computers \& Geosciences 54:361-376

Viterbo E, Boutros J (1999) A Universal Lattice Code Decoder for Fading Channels. IEEE Transactions on Information Theory 45(5):1639-1642 
Walker JG (1984) Satellite constellations. Journal of the British Interplanetary Society 37:559-572

Walter T, Enge P (1995) Weighted RAIM for precision approach. In: Proceedings of ION GPS 1995, Palm Springs, CA, vol 8, pp 1995-2004

Wang J, Stewart MP, Tsakiri M (1998) A discrimination test procedure for ambiguity resolution on-the-fly. Journal of Geodesy 72(11):644-653

Wang L, Verhagen S (2015) A new ambiguity acceptance test threshold determination method with controllable failure rate. Journal of Geodesy 89(4):361-375

Wei M, Schwarz KP (1995) Fast Ambiguity Resolution Using an Integer Nonlinear Programming Method. In: Proceedings of ION GPS 1995, Palm Springs, CA, USA, pp 1101-1110

Wen Z, Henkel P, Günther C (2011) Reliable estimation of phase biases of GPS satellites with a local reference network. In: Proceedings of IEEE ELMAR 2011, Zadar, Croatia, pp 321-324

Wu JT, Wu SC, Hajj GA, Bertiger WI, M LS (1993) Effects of antenna orientation on GPS carrier phase. Manuscripta Geodaetica 18(2):91-98

Wübben D, Seethaler D, Jaldén J, Matz G (2011) Lattice reduction. IEEE Signal Processing Magazine 28(3):70-91

Wübbena G (1989) The GPS adjustment software package-GEONAP-concepts and models. In: Proceedings of 5th International Symposium on Satellite Positioning, Las Cruces, NM, USA, pp $452-461$

Wübbena G, Schmitz M, Bagge A (2005) PPP-RTK: precise point positioning using state-space representation in RTK networks. In: Proceedings of ION GNSS 2005, Long Beach, CA, USA, pp 2584-2594

Xu P (2001) Random simulation and GPS decorrelation. Journal of Geodesy 75(7):408-423

Xu P (2006) Voronoi Cells, Probabilistic Bounds, and Hypothesis Testing in Mixed Integer Linear Models. IEEE Transactions on Information Theory 52(7):3122-3138

Xu P, Cannon E, Lachapelle G (1995) Mixed integer programming for the resolution of GPS carrier phase ambiguities. Presented at IUGG95 General Assembly, Boulder, CO, USA

Zaminpardaz S, Teunissen PJG, Nadarajah N (2017) IRNSS/NavIC and GPS: a single- and dualsystem L5 analysis. Journal of Geodesy 91(8):915-931

Zhang B, Teunissen PJG, Odijk D (2011) A novel un-differenced PPP-RTK concept. Journal of Navigation 64(S1):180-191

Zhang F (2006) The Schur complement and its applications. Springer Science \& Business Media

Zumberge JF, Heflin MB, Jefferson DC, Watkins MM, Webb FH (1997) Precise point positioning for the efficient and robust analysis of GPS data from large networks. Journal of Geophysical Research: Solid Earth 102(B3):5005-5017 